\title{
SUPRAMOLECULAR SOLAR CELLS
}

Navaneetha Krishnan Subbaiyan, B.Tech

\author{
Dissertation Prepared for the Degree of \\ DOCTOR OF PHILOSOPHY
}

\section{UNIVERSITY OF NORTH TEXAS}

August 2012

APPROVED:

Francis D'Souza, Major Professor Justin Youngblood, Committee Member Mohammad Omary, Committee Member Nigel Shepard, Committee Member William E. Acree, Jr., Chair of the Department of Chemistry Mark Wardell, Dean of the Toulouse Graduate School 
Subbaiyan, Navaneetha Krishnan. Supramolecular Solar Cells. Doctor of Philosophy (Chemistry - Analytical Chemistry), August 2012,196 pp., 6 tables, 85 illustrations, references, 336 titles.

Supramolecular chemistry - chemistry of non-covalent bonds including different type of intermolecular interactions viz., ion-pairing, ion-dipole, dipole-dipole, hydrogen bonding, cation- $\pi$ and Van der Waals forces. Applications based on supramolecular concepts for developing catalysts, molecular wires, rectifiers, photochemical sensors have been evolved during recent years. Mimicking natural photosynthesis to build energy harvesting devices has become important for generating energy and solar fuels that could be stored for future use. In this dissertation, supramolecular chemistry is being explored for creating light energy harvesting devices. Photosensitization of semiconductor metal oxide nanoparticles, such as titanium dioxide $\left(\mathrm{TiO}_{2}\right)$ and tin oxide $\left(\mathrm{SnO}_{2},\right)$, via host-guest binding approach has been explored. In the first part, selfassembly of different porphyrin macrocyclic compounds on $\mathrm{TiO}_{2}$ layer using axial coordination approach is explored. Supramolecular dye sensitized solar cells built based on this approach exhibited Incident Photon Conversion Efficiency (IPCE) of 36\% for a porphyrin-ferrocene dyad. In the second part, surface modification of $\mathrm{SnO}_{2}$ with water soluble porphyrins and phthalocyanine resulted in successful self-assembly of dimers on $\mathrm{SnO}_{2}$ surface. IPCE more than $50 \%$ from $400-700 \mathrm{~nm}$ is achieved for the supramolecular self-assembled heterodimer photocells is achieved. In summary, the axial ligation and ion-pairing method used as supramolecular tools to build photocells, exhibited highest quantum efficiency of light energy conversion with panchromatic 
spectral coverage. The reported findings could be applied to create interacting molecular systems for next generation of efficient solar energy harvesting devices. 
Copyright 2012

by

Navaneetha Krishnan Subbaiyan 


\section{ACKNOWLEDGEMENTS}

I thank Prof. Francis D'Souza for believing in me and I enjoyed the every moment that I have spent and I will always remember his words of wisdom "Just do it I say and Give it a shot." I thank Prof. Justin Youngblood, Prof. Nigel Shepherd and Prof. Mohammad Omary at UNT for accepting my offer warmly to be in my committee and also for their support and sharing their wisdom. Also I'm extremely thankful to professors and the administrative members at UNT for making my chemistry department transfer easier. I thank our collaborators from different universities for letting me work on their projects which helped me to expand my research skills. Sincere thanks to all the faculty members at Wichita State for their wonderful support and encouragement. I thank Prof. Nikolai V. Tkachenko and Prof. Helge Lemmetyinen for providing me the opportunity to learn the art of making inverted organic solar cells. Working experience with my lab mates always filled with fun and really enjoyed every moment of hanging out with them and I thank them truly. I would like to thank all my school teachers and faculties from CECRI, friends and my seniors for their support and encouragement. I express my sincere gratitude to my parents and my entire family for their full support. I would like to acknowledge the funding agencies, NSF, NSFEPSCOR, Tampere University of Technology, Wichita State and University of North Texas for their financial support during my Ph.D. Incredible moment of this time wouldn't be the possible without the people mentioned above. 


\section{TABLE OF CONTENTS}

Page

ACKNOWLEDGEMENTS iii

LIST OF TABLES vii

LIST OF SCHEMES viii







1.2 Biosupramolecular Photosynthetic System .............................................. 2

1.3 Photoinduced Electron Transfer (PET) and Photoelectrochemical Cells (PECs)

1.4 PECs Based on Artificial Photosynthesis .................................................. 15



CHAPTER 2 MATERIALS AND PHYSICAL METHODS ............................................. 37





2.3 Surface Characterization Techniques ………......................................... 41







CHAPTER 3 SUPRAMOLECULAR DONOR-ACCEPTOR HYBRID OF ELECTROPOLYMERIZED ZINC PORPHYRIN WITH AXIALLY COORDINATED











CHAPTER 4 SUPRAMOLECULAR DSSCS BASED ON AXIAL LIGATION ................66

4.1 Surface Modification of Nanocrystalline $\mathrm{TiO}_{2}$ with Coordinating Ligands to








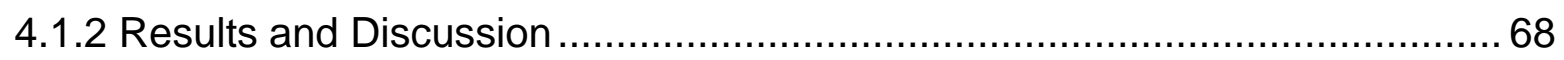

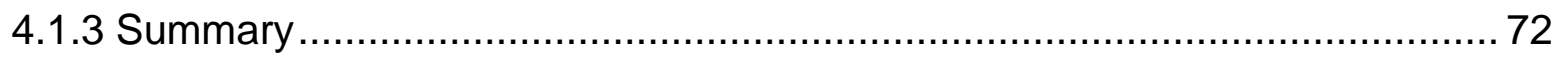

4.2. Effect of Metal Atom and Solvent Optimization................................................ 73

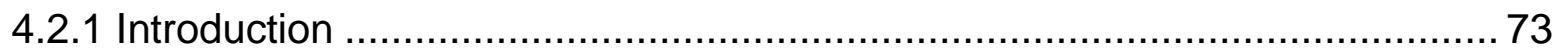

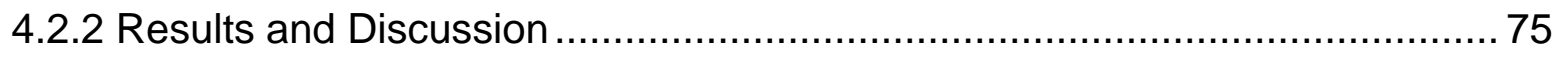

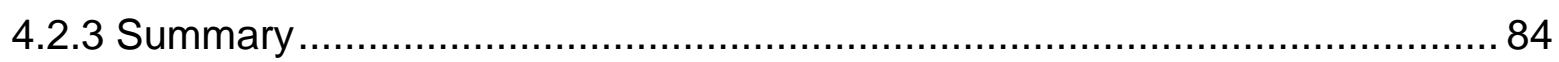

CHAPTER 5 ANION BINDING BASED SUPRAMOLECULAR SYSTEMS .................. 85

5.1 Effect of Anion Binding on Charge Stabilization in a Bis-Fullerene-

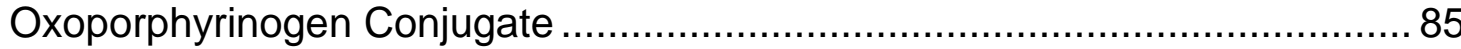

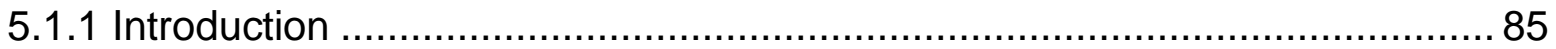

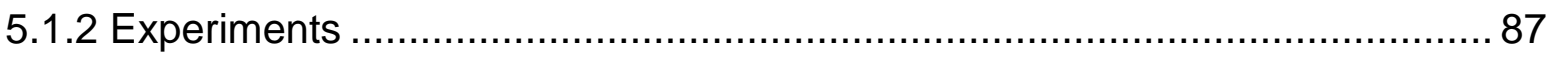





5.2 Anion-Complexation-Induced Stabilization of Charge Separation ..................96

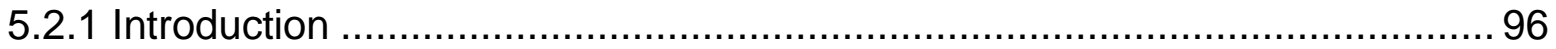







5.3 Porphyrin-Oxoporphyrinogen Surface Modified $\mathrm{TiO}_{2}$ Supramolecular Solar Cell 115

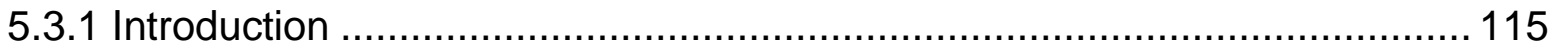



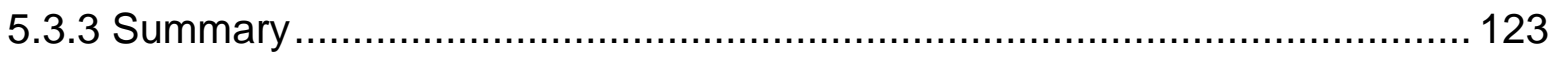

CHAPTER 6 ION-PAIRED DYE-METAL OXIDE SUPRAMOLECULAR SYSTEM ..... 124

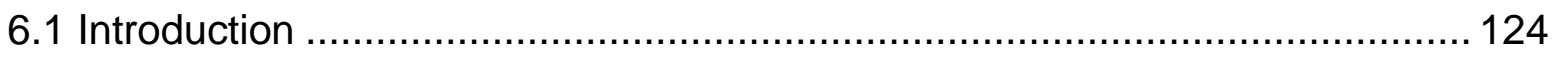

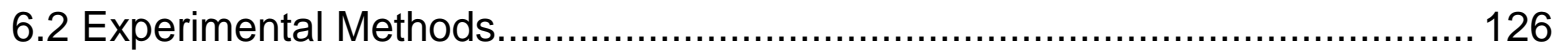

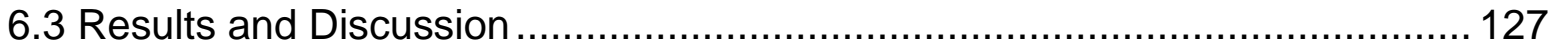

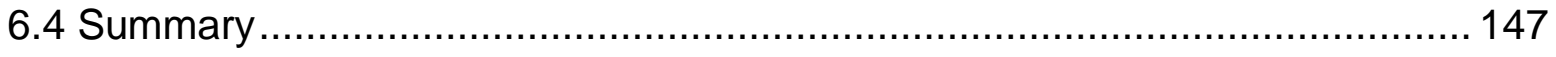

CHAPTER 7 ION-PAIRED HETERODIMER SUPRAMOLECULAR SYSTEM............ 149

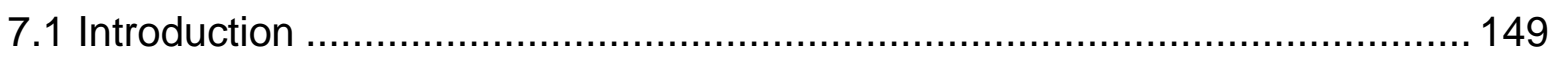

7.2 Results and Discussion ..................................................................... 151 


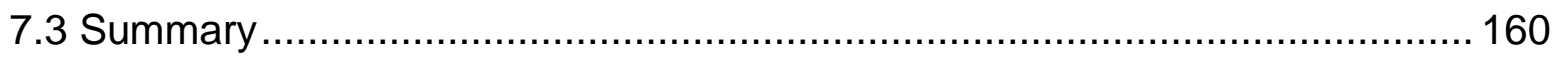

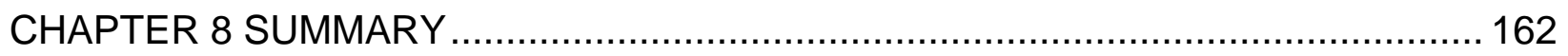

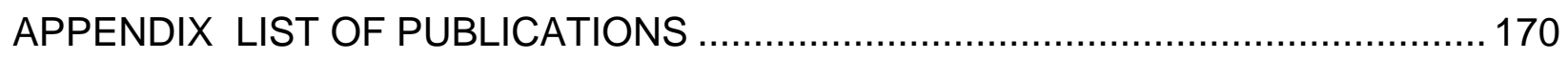

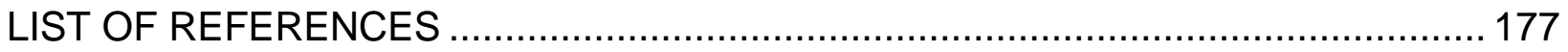




\section{LIST OF TABLES}

Table 4.2.1: Optical and electrochemical properties of MgTPP and ZnTPP. $E_{0-0}$ was calculated from the average of absorbance and emission peak. Excited state oxidation potential $E^{*}{ }_{o x}$ was calculated using the electrochemical oxidation potential $\left(E_{o x}\right) . E_{o x}{ }^{*}=$ $E_{(0-0)}-E_{o x}$

Table 4.2.2: Solvent resistance $\left(R_{s}\right)$, charge transfer resistance $\left(R_{c t}\right)$ and double layer capacitance $\left(C_{d l}\right)$ of the symmetric $\mathrm{Pt}$ electrode configuration of different solvent containing $0.5 \mathrm{M}$ tetra butyl ammonium iodide and $0.05 \mathrm{M} \mathrm{I}_{2}$. 83

Table 5.2.1: Binding constants $(K)$, rate constants of charge separation $\left(k_{C S}\right)$, charge recombination $\left(k_{\mathrm{CR}}\right)$ and lifetimes of CS states $\left(\tau_{\mathrm{RIP}}\right)$ for the investigated supramolecular complexes in o-dichlorobenzene. 102

Table 6.1: Spectral peak position and apparent association constant, Ka and freeenergy change for charge injection of water soluble, cation porphyrins binding to $\mathrm{SnO}_{2}$ nanocrystalline particles in water.

Table 6.2: Performance of the $\mathrm{FTO} / \mathrm{SnO}_{2} / \mathrm{M}(\mathrm{P})(\mathrm{P}=\mathrm{TMPyP}$, TAP or TMIP) solar cells investigated in the present work.

Table 7.1: Performance of the $\mathrm{FTO} / \mathrm{SnO}_{2} / \mathrm{M}(\mathrm{P})$ supramolecular solar cells investigated in the present study. 159 


\section{LIST OF SCHEMES}

Scheme 3.1: Structural formula of tetrakis(4-(N,N-diphenylamino)phenyl) porphyrinatozinc(II), $\left(\mathrm{Ph}_{2} \mathrm{~N}\right)_{4} \mathrm{ZnP}$, used in forming a $\left(\mathrm{Ph}_{2} \mathrm{~N}\right)_{4} \mathrm{ZnP}$ polymer film electrode via electropolymerization, and imidazole-appended fullerene, C60im, to coordinate the zinc porphyrin in the polymer film to ultimately yield a $\left[\left(\mathrm{Ph}_{2} \mathrm{~N}\right)_{4} \mathrm{ZnP}\right.$ polymer film]fullerene donor-acceptor hybrid. 48

Scheme 4.1.1: Structures of the nitrogenous ligand possessing carboxylic acids used to modify the $\mathrm{TiO}_{2}$ surface (1-4); sensitizers (6-7) and the dyad (8) used to axially coordinate the immobilized nitrogenous bases on $\mathrm{TiO}_{2}$ surface. $\mathbf{5}$ is a control compound lacking the axial ligand entity. 67

Scheme 4.2.1: Structure of a) Zinc tetraphenyl porphyrin (ZnTPP) and b) Magnesium tetraphenyl porphyrin (MgTPP) c) imidazole benzoic acid (im) d) 3-fluoropyridine 4carboxylic acid..... 74

Scheme 5.1.1: Structure of the newly synthesized $\operatorname{OXP}\left(\mathrm{C}_{60}\right)_{2}$ triad, to probe anion binding effect on charge separation. 86

Scheme 5.2.1: Structural origin of two-guest reagent/anion cofactor complexation (left figure, peripheral substituents are omitted for clarity) and chemical structure of the bisporphyrin- substituted oxoporphyrinogen, 1 and bis(4-pyridyl)-substituted fullerene used in this work. Axial coordination and anion binding site are also shown for clarity..... 97

Scheme 5.3.1: Schematic figure of the zinc porphyrin-oxoporphyrinogen (ZnP-OXP) surface modification using axial coordination onto the $\mathrm{TiO}_{2} / \mathrm{FTO}$ solar cell to demonstrate enhanced photocurrent generation upon $\mathrm{F}^{-}$binding to OXP. The photochemical electron- and hole- transfer processes are shown by the arrows 117

Scheme 6.1: Structures of the cationic water soluble porphyrins employed to surface modify nanocrystalline $\mathrm{SnO}_{2}$ for photoelectrochemical studies in the present study... 126

Scheme 7.1: Structures of the cationic water soluble porphyrins and anionic phthalocyanine sulfonic acids employed to surface modify nanocrystalline $\mathrm{SnO}_{2}$ for photoelectrochemical studies in the present study. 151 


\section{LIST OF FIGURES}

Figure 1.1: Classification of supramolecular interactions. 2

Figure 1.2: a) Absorption spectra of various energy capturing pigments from algae and plants. b) Energy-transfer cascade for antenna pigments in light-harvesting complexes of the algae Porphyridium cruetum. 3

Figure 1.3: Charge separation at the photosynthetic reaction centre of Rps. Viridis...... 4

Figure 1.4: Mechanism of a) oxidative quenching b) reductive quenching. $S_{0}$ and $S_{1}$ are HOMOs and LUMOs respectively. 8

Figure 1.5: Types of photoelectrochemical cells( ntype, ptype) (a, b) photovoltaic cells $(c, d)$ photoelectrosynthetic cells $(d, e)$ photocatalytic cells ${ }^{9}$. 10

Figure 1.6: Schematic diagram of the photoelectrochemical cell composed of dye (S) sensitized $\mathrm{TiO}_{2}$ semiconductor (n-type), redox mediator $(O \rightleftharpoons R+e-)$ and counter electrode. 12

Figure 1.7: Model $I-V$ curve of the photoelectrochemical cell. 13

Figure 1.8: a) Covalently linked donor-acceptor triad b) Non-covalently linked donoracceptor dyad. 16

Figure 1.9: a) Photoelectrochemical cell based on LB film using fullerene derviatives and arachidic acid. b) Porphyrin fullerene dyad and Poly-Hexyl Thiophene (PHT) used for the formation of LB film.

Figure 1.10: SAMs of fullerene appended monothiol and trithiol on gold electrode surface. 20

Figure 1.11: Co-self assembled monolayer of ferrocene-porphyrin-fullerene triad and boron dipyrin on gold surface. 21

Figure 1.12: Schematic diagram electrophoretic deposition of fullerene on a conductive electrode surface. 22

Figure 1.13: a) Model example of donor-acceptor dyad used for electrophoretic deposition. b) Composite cluster used in different combination along with electrophoretic deposition of fullerene. 23

Figure 1.14: Schematic diagram of photoelectrochemical cell self assembled using ionion interaction between donor and acceptor. 24

Figure 1.15: Schematic diagram of photoelectrochemical cell based on pi-pi interaction between donor and acceptor. 25 
Figure 1.16: Schematic diagram of hydrogen bonding between the fullerene and

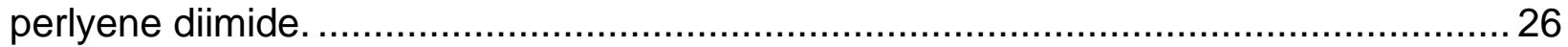

Figure 1.17: a) Axial coordination of fullerene derivative and water soluble zinc porphyrin. b) Axial coordination between fullerene and aluminum porphryin. 28

Figure 1.18 a) Donor- acceptor based organic dye for dye sensitization. b) Porphyrin based carboxylic acid dye used for dye sensitized solar cells. 30

Figure 3.1: Curves of (a) multi-scan cyclic voltammetry as well as the potential dependence of (b) resonant frequency change, and (c) dynamic resistance changes for electropolymerization of a $\left(\mathrm{Ph}_{2} \mathrm{~N}\right)_{4} \mathrm{ZnP}$ polymer film from a 1,2-dichlorobenzene solution of $0.124 \mathrm{mM}$ tetrakis(4-(N,N-diphenylamino)phenyl)porphyrinatozinc(II) and $0.1 \mathrm{M}$ (TBA) $\mathrm{ClO}_{4}$. Potential sweep rate was $100 \mathrm{mV} / \mathrm{sec}$. A 5-mm diameter gold-film electrode of the $10-\mathrm{mHz} \mathrm{Au} / \mathrm{Ti}$ quartz crystal resonator served as the working electrode.

51

Figure 3.2: (a) Flow-injection analysis measurements with the EQCM detection of the $\mathrm{C}_{60}$ im binding to the $\left(\mathrm{Ph}_{2} \mathrm{~N}\right)_{4} \mathrm{ZnP}$ polymer film to form the donor-acceptor dyad. Sample volume of each injection of $17.4 \mathrm{mM} \mathrm{C60im}$ in 1,2-dichlorobenzene was $1 \mathrm{~mL}$. Flow rate was $50 \mu \mathrm{L} / \mathrm{min}$. The frequency change accompanying initial three consecutive injections are shown. (b) Cyclic voltammograms at the 1.6-mm diameter Au disk electrode for (1) $0.5 \mathrm{mM} \mathrm{C60im,} \mathrm{(2)}\left(\mathrm{Ph}_{2} \mathrm{~N}\right)_{4} \mathrm{ZnP}$ polymer film and (3) C60im bound to the $\left(\mathrm{Ph}_{2} \mathrm{~N}\right)_{4} \mathrm{ZnP}$ polymer film demonstrating formation of an electro-active $\left[\left(\mathrm{Ph}_{2} \mathrm{~N}\right)_{4} \mathrm{ZnP}\right.$ polymer film]-C60im donor-acceptor dyad in $0.1 \mathrm{M}(\mathrm{TBA}) \mathrm{ClO}_{4}$, in 1,2-dichlorobenzene. Potential scan rate was $100 \mathrm{mV} / \mathrm{s}$. 53

Figure 3.3: Atomic force microscopy tapping mode $1 \times 1 \mu \mathrm{m}$ image of (a) the $\left(\mathrm{Ph}_{2} \mathrm{~N}\right)_{4} \mathrm{ZnP}$ polymer film and (b) the $\left(\mathrm{Ph}_{2} \mathrm{~N}\right)_{4} \mathrm{ZnP}$ polymer film with the bound $\mathrm{C}_{60} \mathrm{im}$ ligand, deposited by electropolymerization on an Au/Cr-coated glass slide. 54

Figure 3.4: Spectra of (a) optical absorption and (b) fluorescence for the $\left(\mathrm{Ph}_{2} \mathrm{~N}\right)_{4} \mathrm{ZnP}$ polymer film deposited on the ITO electrode (1 and 1') before and (2 and 2') after coordinating $\mathrm{C}_{60} \mathrm{im}$. $\lambda_{\mathrm{ex}}=444 \mathrm{~nm}$ 56

Figure 3.5: (a) Absorption spectrum of $\left(\mathrm{Ph}_{2} \mathrm{~N}\right)_{4} \mathrm{ZnP}$ in o-dichlorobenzene. (b) Emission

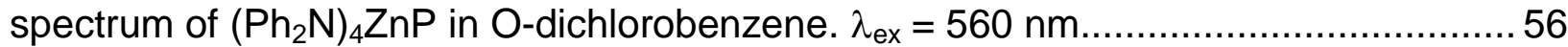

Figure 3.6: Photocurrent-photovoltage characteristics for the $\left(\mathrm{Ph}_{2} \mathrm{~N}\right)_{4} \mathrm{ZnP}$ polymer film coated ITO electrode (in the absence of $\mathrm{C}_{60}$ ) in acetonitrile solutions, which were: (1) 92, (2) 96 , (3) 101, (4) 105 , (5) 110 , and (6) $119 \mathrm{mM}$ in $\mathrm{I}_{2}$ and $0.1 \mathrm{M}$ in iodide stability. 58

Figure 3.7: Absorbance spectrum of $\left(\mathrm{Ph}_{2} \mathrm{~N}\right)_{4} \mathrm{ZnP}$ polymer film on ITO electrode obtained under different scan rates; (i) $25 \mathrm{mV} / \mathrm{s}$, (ii) $50 \mathrm{mV} / \mathrm{s}$ and (iii) $75 \mathrm{mV} / \mathrm{s}$ in odichlorobenzene containing $0.1 \mathrm{M}(\mathrm{TBA}) \mathrm{ClO}_{4}$ 60

Figure 3.8: Photocurrent-photovoltage characteristics for electrodes modified with the $\left(\mathrm{Ph}_{2} \mathrm{~N}\right)_{4} \mathrm{ZnP}$ polymer film of different thickness, prepared by scanning the potential at (1) 
25, (2) 50 , and (3) $75 \mathrm{mV} / \mathrm{s}$ during film deposition (10 CV cycles at $25 \mathrm{mV} / \mathrm{s}$ ). The photocurrent-photovoltage curves were generated for the acetonitrile solution of the $\mathrm{I}_{3}$ / $I^{-}(0.1 \mathrm{M} / 0.1 \mathrm{M})$ mediating redox couple. 60

Figure 3.9: Effect of light switching on (a) photocurrent and (b) photovoltage of the $\left(\mathrm{Ph}_{2} \mathrm{~N}\right)_{4} \mathrm{ZnP}$ polymer film coated ITO electrode in the (1 and 1') absence and (2 and 2') presence of $\mathrm{C}_{60} \mathrm{im}$. The photocurrent and photovoltage switching were recorded in acetonitrile solution containing $\mathrm{I}_{3} / \mathrm{I}^{-}(0.1 \mathrm{M} / 0.1 \mathrm{M})$ mediating redox couple 62

Figure 3.10: Photocurrent-photovoltage (I-V) characteristics for electrodes modified with the $\left(\mathrm{Ph}_{2} \mathrm{~N}\right)_{4} \mathrm{ZnP}$ polymer film (1) in the absence and (2) presence of $\mathrm{C}_{60} \mathrm{im}$. The $I-V$ curves were generated for the acetonitrile solution of the $\mathrm{I}_{3}{ }^{-} / \mathrm{I}^{-}(0.1 \mathrm{M} / 0.1 \mathrm{M})$ mediating redox couple. 62

Figure 3.11: (a) Incident photon-to-current conversion efficiency (IPCE) for the $\left(\mathrm{Ph}_{2} \mathrm{~N}\right)_{4} \mathrm{ZnP}$ polymer film coated ITO electrode (10 cycles at $\left.25 \mathrm{mV} / \mathrm{s}\right)$ in (1) absence and (2) presence of the coordinated $\mathrm{C}_{60}$ im ligand, in acetonitrile solution of the $\mathrm{I}_{3}{ }^{-} / \mathrm{I}^{-}(0.1$ $\mathrm{M} / 0.1 \mathrm{M}$ ) mediating redox couple. (b) Schematic view of the mechanism of the cathodic photocurrent generation by the $\left(\mathrm{Ph}_{2} \mathrm{~N}\right)_{4} \mathrm{ZnP}$ polymer film]-fullerene modified optically transparent ITO electrode 64

Figure 4.1.1: a) UV-visible spectrum of the sensitizer and dyad immobilized on 4 modified $\mathrm{FTO} / \mathrm{TiO}_{2}$ electrodes after correcting for $\mathrm{FTO} / \mathrm{TiO}_{2}$ absorbance. b) UV-Visible spectrum of the sensitizers and the dyad employed in the present study. 68

Figure 4.1.2: (a) Pictures of sensitizers in solution and on the $\mathrm{FTO}^{-\mathrm{TiO}_{2}}$ modified with 4 or 5 electrodes, (b) $I-V$ characteristics showing the effect of different sensitizers bound to 4 modified $\mathrm{TiO}_{2}$, and (c) $I-V$ characteristics showing the effect of different surface modifiers on the photoelectrochemical behavior upon binding 8, and (d) IPCE curves of for the electrodes shown in (c). $\mathrm{I}-\mathrm{V}$ curves were generated in DCB containing $\mathrm{I}_{3}^{-} / \mathrm{I}^{-}(0.5$ $\mathrm{M} / 0.03 \mathrm{M}$ ) redox mediator using an AM 1.5 simulated light source with a $340 \mathrm{~nm}$ UVcut off filter. The black lines in $b$ and $c$ are dark currents recorded for the 4:8 modified electrodes. 69

Figure 4.1.3: a) IPCE curves for 4:8, 4:7 and 4:6 modified $\mathrm{TiO}_{2} / \mathrm{FTO}$ in $\mathrm{DCB}$ containing $\mathrm{I}_{3}{ }^{-} / \mathrm{I}^{-}(0.5 \mathrm{M} / 0.03 \mathrm{M})$ redox mediator. b) Effect of light switching on photocurrent of the studied systems immobilized onto $\mathrm{TiO}_{2} /$ FTO surface. The switching experiments were performed in DCB containing a $0.5 \mathrm{M}(\mathrm{n}-\mathrm{Bu})_{4} \mathrm{NI}$ and $0.03 \mathrm{M} \mathrm{I}_{2}$ as redox mediator. c) Schematics of zinc porphyrin-ferrocene dyad (8) surface modified via axial coordination of 4 immobilized onto the $\mathrm{TiO}_{2}$ film on the FTO electrode. The photochemical and redox processes are shown by arrows. 71

Figure 4.2.1: a) UV absorbance of the compounds measured in o-dichlorobenzene (DCB). b) Steady state emission spectrum of the compounds excited at their corresponding Soret band in ODB solvent. c) UV absorbance of the compounds after axially coordinating with the imidazole immobilized $\mathrm{TiO}_{2}$. d) Differential Pulse 
Voltammogram of the compounds measured in $0.1 \mathrm{M}$ Tetra butyl ammonium perchlorate in DCB. 76

Figure 4.2.2: (a)-(b) HOMO-LUMO of ZnTPP and (c)-(d) HOMO- LUMO of MgTPP axially coordinating with imidazole benzoic acid. B3LYP/3-21G $\left({ }^{*}\right)$ optimized structures. 78

Figure 4.2.3: (a) Photocurrent switching of MgTPP and $\mathrm{ZnTPP}$ immobilized on $\mathrm{TiO}_{2}$. (b) Photocurrent switching of MgTPP adsorbed different coordinating ligands on $\mathrm{TiO}_{2}$. (c) $\mathrm{J}$ $V$ characeristics of MgTPP and ZnTPP immobilized on $\mathrm{TiO}_{2}$. (d) IPCE plot of MgTPP and ZnTPP immobilized on $\mathrm{TiO}_{2}$. All of the above experiments were performed in 0.5 $\mathrm{TBAl} / 0.05 \mathrm{M} \mathrm{I}_{2}$ in ODB. 80

Figure 4.2.4: Light Harvesting Efficiency ${ }^{176}$ of the $\mathrm{MgTPP} / \mathrm{im}-\mathrm{TiO}_{2}$ and $\mathrm{ZnTPP} / \mathrm{im}-\mathrm{TiO}_{2}$ electrode. 81

Figure 4.2.5: (a) $\mathrm{J}-\mathrm{V}$ plot of $\mathrm{MgTPP} / \mathrm{im}-\mathrm{TiO}_{2}$ in different electrolyte solvent conditions (b) Effect of electrolyte solvent conditions on the current density and fill factor of MgTPP/im$\mathrm{TiO}_{2}$ electrode. (c) Bode plot of impedance and phase angle vs log frequency of the two platinized FTO under symmetric electrode condition using different electrolyte compositions. (d) Equivalent circuit used for fitting the impedance data $R s$ - solution resistance, $R_{c t}$ - charge transfer resistance for at Pt/electrolyte interface and $C_{d l}$ is the double capacitance of the same interface. All of the above experiments were performed in mentioned solvents with $0.5 \mathrm{TBAl} / 0.05 \mathrm{M} \mathrm{I}_{2}$ as redox mediator. 82

Figure 5.1.1: (a) Absorption spectra of $\operatorname{OXP}\left(\mathrm{C}_{60}\right)_{2}$ triad $(7.5 \mu \mathrm{M})$ upon increasing addition of $\mathrm{F}^{-}(0.1-0.5$ eq.). (b) Benesi-Hildebrand plot constructed to obtain the binding constant, and (c) mole ratio plot to obtain the molecular stoichiometry of the $\operatorname{OXP}\left(\mathrm{C}_{60}\right)_{2}$ triad: $\mathrm{F}^{-}$complex in DCB. 90

Figure 5.1.2: (a) Fluorescence spectra of $\operatorname{OXP}\left(\mathrm{C}_{60}\right)_{2}$ triad $(7.5 \mu \mathrm{M})$ on increasing addition of $\mathrm{F}^{-}\left(0.1-0.5\right.$ eq.) in DCB. $\lambda_{\mathrm{ex}}=509 \mathrm{~nm}$. (b) Cyclic voltammograms of $\operatorname{OXP}\left(\mathrm{C}_{60}\right)_{2}$ triad in the absence (dark line) and presence (red line) of $\mathrm{F}^{-}$in deaerated DCB containing $0.1 \mathrm{M}(\mathrm{TBA}) \mathrm{ClO}_{4}$. Scan rate $=100 \mathrm{mV} / \mathrm{s}$. 91

Figure 5.1.3: Femtosecond transient absorption spectra of $\operatorname{OXP}\left(\mathrm{C}_{60}\right)_{2}$ triad: $\mathrm{F}^{-}$in deaerated DCB. $\lambda_{\mathrm{ex}}=430 \mathrm{~nm}$. The figure inset shows decay of the $1000 \mathrm{~nm}$ transient band corresponding to fullerene radical anion. 93

Figure 5.1.4: (a) Nanosecond transient absorption spectra of $\operatorname{OXP}\left(\mathrm{C}_{60}\right)_{2}$ triad: $\mathrm{F}^{-}$in deaerated DCB. Inset shows the time profile of the $\mathrm{C}_{60^{-}}$at $1000 \mathrm{~nm}$. (b) Nanosecond transient absorption spectra obtained by $430 \mathrm{~nm}$ laser light of 1 in deaerated DCB.inset shows time profile of $\mathrm{C}_{60}{ }^{-}$band at $1000 \mathrm{~nm}$. (c) Energy level diagram showing the electron transfer processes of $\operatorname{OXP}\left(\mathrm{C}_{60}\right)_{2}$ triad: $\mathrm{F}^{-}$via the singlet and triplet $\mathrm{OXP}$ in deaerated DCB. 94 
Figure 5.2.1: Optical absorption spectral changes observed during (a) binding of $\mathrm{Py}_{2} \mathrm{C}_{60}$ to 1 to form 1. $\mathrm{Py}_{2} \mathrm{C}_{60}$ complex, and (b) F- binding to OXP of the $1 \mathrm{Py}_{2} \mathrm{C}_{60}$ complex to form the $1\left(\mathrm{~F}^{-}\right)$. $\mathrm{Py}_{2} \mathrm{C}_{60}$ complex in o-dichlorobenzene. The figure insets show BenesiHildebrand plots constructed to obtain the binding constants; $A_{\circ}$ and $d A$ represent respectively absorbance values in the absence of added guest and change in absorbance upon guest binding. ....................................................................... 100

Figure 5.2.2: (a) Optical absorption spectral changes observed during acetate binding to $1-\mathrm{Py}_{2} \mathrm{C}_{60}$ to form the $\mathbf{1}\left(\mathrm{CH}_{3} \mathrm{COO}^{-}\right)$. $\mathrm{Py}_{2} \mathrm{C}_{60}$ complex in o-dichlorobenzene.(b) Optical absorption spectral changes observed during dihydrogen phosphate binding to 1 . $\mathrm{Py}_{2} \mathrm{C}_{60}$ to form the $1\left(\mathrm{H}_{2} \mathrm{PO} 4^{-}\right)$. $\mathrm{Py}_{2} \mathrm{C}_{60}$ complex in o-dichlorobenzene. The figure inset shows a Benesi-Hildebrand plot constructed to obtain the binding constant. (c) Optical absorption spectral changes observed during perchlorate binding to 1. $\mathrm{Py}_{2} \mathrm{C}_{60}$ to form the 1 (ClO4-). $\mathrm{Py}_{2} \mathrm{C}_{60}$ complex. Spectrum (i) represents that of 1 and (ii) represents that of 1. $\mathrm{Py}_{2} \mathrm{C}_{60}$ in o-dichlorobenzene. No change in the $513 \mathrm{~nm}$ band of OXP upon addition of $\mathrm{ClO}_{4}^{-}$indicate little or no interaction. .................................................. 101

Figure 5.2.3: (a) ${ }^{1} \mathrm{H}$ NMR titration of 1 with up to 1 equivalent of $\mathrm{Py}_{2} \mathrm{C}_{60}$ in 0 dichlorobenzene-d4. Note upfield shift of resonances of $\mathrm{Py}_{2} \mathrm{C}_{60}$ due to shielding by porphyrin aromatic m-electronic system. Assignments of relevant peaks are shown in the lower part of the figure. (b) Titration of the 1. $\mathrm{Py}_{2} \mathrm{C}_{60}$ complex formed in (a) with fluoride anions (tetra-n-butylammonium fluoride solution in o-dichlorobenzene-d4). Asterisk denotes porphyrinogen $\mathrm{NH}$ resonance. Note the disappearance of the peak labeled with an asterisk due to hydrogen bonding to fluoride anions. 103

Figure 5.2.4: a) Cyclic voltammograms of (i) 1, (ii) 1. $\mathrm{Py}_{2} \mathrm{C}_{60}$ obtained by equimolar mixture of 1 and $\mathrm{Py}_{2} \mathrm{C}_{60}$, and (iii) 1(F-). $\mathrm{Py}_{2} \mathrm{C}_{60}$ obtained by equimolar mixture of 1 . $\mathrm{Py}_{2} \mathrm{C}_{60}$ and $\mathrm{F}^{-}$. b) Cyclic voltammograms of (i) OXP-(bz) $)_{2}$ and (ii) $\mathrm{Py}_{2} \mathrm{C}_{60}$ in Ar-saturated o-dichlorobenzene containing $0.1 \mathrm{M}\left(\mathrm{n}-\mathrm{Bu}_{4} \mathrm{~N}\right) \mathrm{ClO}_{4}$. Scan rate $=0.1 \mathrm{~V} / \mathrm{s}$. The asterisk $\left({ }^{*}\right)$ represents ferrocene redox couple added as an internal standard. c) Cyclic Voltammograms of $\mathrm{OXP}(\mathrm{Bz})_{2}$ on (i) 0 eq. (ii) 1.0 eq. and (iii) 2.0 eq. addition of ( $\mathrm{n}$ $\left.\mathrm{Bu}_{4} \mathrm{~N}\right) \mathrm{F}^{-}$and in o-dichlorobenzene containing $0.1 \mathrm{M}\left(\mathrm{n}-\mathrm{Bu}_{4} \mathrm{~N}\right) \mathrm{PF}_{6}$. Scan rate $=100$ $\mathrm{mV} / \mathrm{s}$. All measurements performed in o-dichlorobenzene containing $0.1 \mathrm{M}\left(\mathrm{n}-\mathrm{Bu}_{4} \mathrm{~N}\right) \mathrm{PF}_{6}$. 105

Figure 5.2.5: Steady-state fluorescence spectrum of (a) 1 in on increasing addition of $\mathrm{Py}_{2} \mathrm{C}_{60}$ to form the 1. $\mathrm{Py}_{2} \mathrm{C}_{60}$ complex (1.1 eq total), and (b) on addition of $\mathrm{F}^{-}$(1.1 eq. total) to the solution of $1 \mathrm{Py}_{2} \mathrm{C}_{60}$ to form the $\mathbf{1}(\mathrm{F}-)$. $\mathrm{Py}_{2} \mathrm{C}_{60}$ complex in o-dichlorobenzene. The samples were excited at $440 \mathrm{~nm}$ corresponding to $\mathrm{ZnP}$ Soret band and the initial concentration of 1 was held at $50 \mu \mathrm{M}$. Spectrum (i) represents that of 1 in the absence of guest entities, (ii) represents that of 1 . $\mathrm{Py}_{2} \mathrm{C}_{60}$ complex, and (iii) represents that of 1(F-). $\mathrm{Py}_{2} \mathrm{C}_{60}$ complex. 107

Figure 5.2.6: (a) Transient absorption spectrum of $\mathbf{1}\left(\mathrm{F}^{-}\right)$. $\mathrm{Py}_{2} \mathrm{C}_{60}$ in o-dichlorobenzene (concentration of each component: $1.5 \times 10^{-4} \mathrm{M}$ ) at $2 \mathrm{ps}$ after femtosecond laser excitation at $440 \mathrm{~nm}$. (b) Decay of absorbance at $910 \mathrm{~nm}$ corresponding to the singlet excited state of the $\mathrm{ZnP}$ moiety in the supramolecular complex. 109 
Figure 5.2.7: (a) Transient absorption spectrum of $1\left(\mathrm{CH}_{3} \mathrm{COO}^{-}\right)$. $\mathrm{Py}_{2} \mathrm{C}_{60}$ in 0 dichlorobenzene (concentration of each component: $1.5 \times 10-4 \mathrm{M}$ ) at 2 ps (black), 500 ps (red), 3000 ps (blue) after femtosecond laser excitation at $440 \mathrm{~nm}$. (b) Decay of absorbance at $910 \mathrm{~nm}$ corresponding to the singlet excited state of the ZnP moiety in the supramolecular complex. 110

Figure 5.2.8: (a) Transient absorption spectrum of $1\left(\mathrm{~F}^{-}\right) \cdot \mathrm{Py}_{2} \mathrm{C}_{60}$ in o-dichlorobenzene (concentration of each component: $3.0 \times 10^{-5} \mathrm{M}$ ) at $1 \mu \mathrm{s}$ after nanosecond laser excitation at $450 \mathrm{~nm}$. (b) Decay of absorbance for $1\left(\mathrm{~F}^{-}\right) \cdot \mathrm{Py}_{2} \mathrm{C}_{60}$ at $1000 \mathrm{~nm}$ corresponding to fullerene anion radical. Inset: 0-8 $\mu$ s time range (c) Decay of absorbance for $\mathbf{1}\left(\mathrm{CH}_{3} \mathrm{COO}^{-}\right) \cdot \mathrm{Py}_{2} \mathrm{C}_{60}$ at $1000 \mathrm{~nm}$. (d) Decay of absorbance for

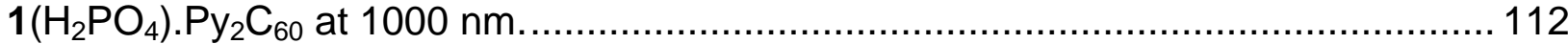

Figure 5.2.9: Plot of the quantum yield of the CS state of $1\left(\mathrm{~F}^{-}\right) . \mathrm{Py}_{2} \mathrm{C}_{60}[\Phi(\mathrm{CS})]$ vs concentration of equimolar $\mathbf{1}\left(\mathrm{F}^{-}\right)$and $\mathrm{Py}_{2} \mathrm{C} 60$ in o-dichlorobenzene 114

Figure 5.3.1: Normalized absorption spectra of (i) ZnP-OXP dyad, (ii) ZnTPP and (iii) OXP in DCB. 118

Figure 5.3.2: Absorption spectra of the ZnP-OXP dyad $(1.92 \mu \mathrm{M})$ upon increasing addition of (a) phenylimidazole axial coordinating ligand: (b) $\mathrm{F}^{-}(0.1-0.5$ eq.) in DCB: (c) Spectral changes observed during titration of $\mathrm{F}^{-}$into a solution of zinc tetraphenylporphyrin (ZnTPP) in DCB. The Benesi-Hildebrand plots constructed to obtain the binding constants for each equilibrium process are shown in the inset of the respective figures. 119

Figure 5.3.3: Differential pulse voltammograms of ZnP-OXP $(0.13 \mathrm{mM})$ (i) in the absence and (ii) and (iii) in the presence of $\mathrm{F}^{-}(1.3$ and $1.5 \mathrm{mM}$.) in deaerated $\mathrm{DCB}$ containing $0.10 \mathrm{M}(\mathrm{TBA}) \mathrm{ClO}_{4}$. Scan rate $=5 \mathrm{mV} / \mathrm{s}$, pulse width $=0.25 \mathrm{~s}$, pulse height $=$ $0.025 \mathrm{~V}$. 120

Figure 5.3.4: (a) $I-V$ and (b) IPCE(\%) characteristics revealing the effect of $\mathrm{F}^{-}$binding to $\mathrm{ZnP}$-OXP dyad (curves (i) in the absence and (ii) in the presence of $0.01 \mathrm{M} \mathrm{F}^{-}$). I-V curves were generated in DCB containing $\mathrm{I}_{3}^{-} / \mathrm{I}^{-}(0.50 \mathrm{M} / 0.050 \mathrm{M})$ redox mediator using an AM 1.5 simulated light source with a $340 \mathrm{~nm}$ UV-cut off filter. Figure b inset shows on-off light switching to demonstrate robustness of the studied electrode. 123

Figure 6.1: Absorption spectra of the (a) free-base and (b) zinc derivatives of (i) TMPyP, (ii) TAP and (iii) TMIP porphyrin derivatives normalized to the Soret band intensity 128

Figure 6.2: Absorption spectral changes observed for (TPA) $\mathrm{H}_{2}$ on increasing addition of colloidal $\mathrm{SnO}_{2}(5-10 \mu \mathrm{L}$ of $1.5 \mathrm{~g} / \mathrm{l}$ each addition) in water (left panel). Absorption spectra recorded with two-beam spectrometer during addition of aqueous solution of (TPA) $\mathrm{H}_{2}(5$ $\mu \mathrm{L}$ of $1.43 \mathrm{mM}$ each addition) to a $\mathrm{SnO}_{2}(3 \mathrm{ml}$ of $0.408 \mathrm{~g} / \mathrm{l})$ solution in water (right panel). $\delta A=A-A o$, where $A$ and $A o$ corresponds to the absorbance of (TPA) $\mathrm{H}_{2}$ in the presence and absence of $\mathrm{SnO}_{2}$. Figure inset in the right panel shows Benesi- 
Hildebrand plot showing linear dependence of the inverse $\delta \mathrm{A}$ at $427 \mathrm{~nm}$ on the inverse of the (TPA) $\mathrm{H}_{2}$ concentration

Figure 6.3: (a) Absorption spectral changes observed for (TMPyP)Zn on increasing addition of colloidal $\mathrm{SnO}_{2}(5-10 \mu \mathrm{L}$ of $1.2 \mathrm{~g} / \mathrm{l}$ each addition) in water. (b) Absorption spectra recorded with two-beam spectrometer during addition of aqueous solution of (TMPyP)Zn ( $5 \mu \mathrm{L}$ of $1.41 \mathrm{mM}$ each addition) to a $\mathrm{SnO}_{2}(3 \mathrm{ml}$ of $0.42 \mathrm{~g} / \mathrm{l})$ solution in water. $\delta A=A-A o$, where $A$ and Ao corresponds to the absorbance of (TMPyP) $Z n$ in the presence and absence of $\mathrm{SnO}_{2}$. Figure $2 \mathrm{~b}$ inset shows Benesi-Hildebrand plot showing linear dependence of the inverse $\delta A$ at $459 \mathrm{~nm}$ on the inverse of the (TMPyP)Zn concentration. 130

Figure 6.4: Absorption spectral changes observed for (TMIP) $\mathrm{H}_{2}$ on increasing addition of colloidal $\mathrm{SnO}_{2}(5-10 \mu \mathrm{L}$ of $1.5 \mathrm{~g} /$ l each addition) in water (left panel). Absorption spectra recorded with two-beam spectrometer during addition of aqueous solution of (TMIP) $\mathrm{H}_{2}\left(5 \mu \mathrm{L}\right.$ of $1.02 \mathrm{mM}$ each addition) to a $\mathrm{SnO}_{2}(3 \mathrm{ml}$ of $0.412 \mathrm{~g} / \mathrm{l})$ solution in water (right panel). $\delta \mathrm{A}=\mathrm{A}-\mathrm{Ao}$, where $\mathrm{A}$ and $\mathrm{Ao}$ corresponds to the absorbance of $(\mathrm{TMIP}) \mathrm{H}_{2}$ in the presence and absence of $\mathrm{SnO}_{2}$. Figure right inset shows Benesi-Hildebrand plot showing linear dependence of the inverse $\delta A$ at $428 \mathrm{~nm}$ on the inverse of the (TMIP) $\mathrm{H}_{2}$ concentration 131

Figure 6.5: Absorption spectral changes observed for (TAP)Zn on increasing addition of colloidal $\mathrm{SnO}_{2}(5-10 \mu \mathrm{L}$ of $1.2 \mathrm{~g} / \mathrm{l}$ each addition) in water (left panel). Absorption spectra recorded with two-beam spectrometer during addition of aqueous solution of (TAP) Zn (5 $\mu \mathrm{L}$ of $1.3 \mathrm{mM}$ each addition) to a $\mathrm{SnO}_{2}(3 \mathrm{ml}$ of $0.40 \mathrm{~g} / \mathrm{l})$ solution in water (right panel). $\delta \mathrm{A}=\mathrm{A}-\mathrm{Ao}$, where $\mathrm{A}$ and Ao corresponds to the absorbance of (TAP)Zn in the presence and absence of $\mathrm{SnO}_{2}$. Figure right inset shows Benesi-Hildebrand plot showing linear dependence of the inverse $\delta \mathrm{A}$ at $431 \mathrm{~nm}$ on the inverse of the (TAP)Zn concentration 131

Figure 6.6: (a) Absorption spectral changes observed for (TMPyP) $\mathrm{H}_{2}$ (3 $\mathrm{ml}$ of $2.17 \mathrm{mM}$ ) on increasing addition of colloidal $\mathrm{SnO} 2(5-10 \mu \mathrm{L}$ of $11.2 \mathrm{mM}$ each addition) in water. (b) Absorption spectra recorded with two-beam spectrometer during addition of aqueous solution of (TMPyP) $\mathrm{H}_{2}\left(5 \mu \mathrm{L}\right.$ of $1.4 \mathrm{mM}$ each addition) to a $\mathrm{SnO}_{2}(3 \mathrm{ml}$ of $0.85 \mathrm{~g} / \mathrm{L})$ solution in water. $\delta A=A-A o$, where $A$ and Ao corresponds to the absorbance of $(\mathrm{TMPyP}) \mathrm{H}_{2}$ in the presence and absence of $\mathrm{SnO}_{2}$. Figure $6.6 \mathrm{~b}$ inset shows BenesiHildebrand plot showing linear dependence of the inverse $\delta A$ at $449 \mathrm{~nm}$ on the inverse of the (TMPyP) $\mathrm{H}_{2}$ concentration. 132

Figure 6.7: Fluorescence spectra of (TMIP) $\mathrm{H}_{2}$ on increasing addition nanocrystalline $\mathrm{SnO}_{2}\left(5-20 \mu \mathrm{L}\right.$ of $1.5 \mathrm{~g} / \mathrm{l}$ solution addition) (left panel). $\lambda_{\mathrm{ex}}=517 \mathrm{~nm}$. Fluorescence decay profiles of (TMIP) $\mathrm{H}_{2}$ in the absence (i) and presence (ii) of nanocrystalline $\mathrm{SnO}_{2}$, excited at $561 \mathrm{~nm}$ using a nano-LED source (right panel). The lamp profile is shown in dashed line. 134 
Figure 6.8: (a) Fluorescence spectra of $(\mathrm{TAP}) \mathrm{H}_{2}$ on increasing addition nanocrystalline $\mathrm{SnO}_{2}\left(5-20 \mu \mathrm{L}\right.$ of $1.5 \mathrm{~g} / \mathrm{l}$ solution addition) (left panel). $\lambda_{\mathrm{ex}}=517 \mathrm{~nm}$. (b) Fluorescence decay profiles of $(\mathrm{TAP}) \mathrm{H}_{2}$ in the absence (i) and presence (ii) of nanocrystalline $\mathrm{SnO}_{2}$, excited at $561 \mathrm{~nm}$ using a nano-LED source (right panel). The lamp profile is shown in dashed line. 135

Figure 6.9: Fluorescence spectra of (TAP) $\mathrm{Zn}(2.17 \mu \mathrm{M})$ on increasing addition nanocrystalline $\mathrm{SnO}_{2}\left(5-20 \mu \mathrm{L}\right.$ of $1.2 \mathrm{~g} /$ solution addition) (left panel). $\quad \lambda_{\text {ex }}=563 \mathrm{~nm}$. Fluorescence spectra of (TMPyP)Zn $(2.17 \mu \mathrm{M})$ on increasing addition nanocrystalline $\mathrm{SnO}_{2}\left(5-20 \mu \mathrm{L}\right.$ of $1.2 \mathrm{~g} / \mathrm{l}$ solution addition) (left panel). $\lambda_{\mathrm{ex}}=563 \mathrm{~nm}$. (right panel)... 135

Figure 6.10: (a) Fluorescence spectra of $(\mathrm{TMPyP}) \mathrm{H}_{2}(3 \mathrm{ml}$ of $2.17 \mu \mathrm{M})$ on increasing addition nanocrystalline $\mathrm{SnO}_{2}$ (addition of $5-20 \mu \mathrm{L}$ solution of $1.1 \mathrm{~g} / \mathrm{L}$ concentration). $\lambda_{\text {ex }}$ $=517 \mathrm{~nm}$. (b) Fluorescence decay profiles of $(\mathrm{TMPyP}) \mathrm{H}_{2}$ in the absence (i) and presence (ii) of nanocrystalline $\mathrm{SnO}_{2}$, excited at $561 \mathrm{~nm}$ using a nano-LED source. The lamp profile is shown in dashed line. Time calibration factor for each channel $=8.77 \mathrm{x}$ $10^{-10} \mathrm{~s}$. 138

Figure 6.11: (a) (i) Fluorescence intensity of the $660 \mathrm{~nm}$ band of (TMPyP)Zn on increasing addition of nanocrystalline $\mathrm{SnO}_{2}$ in water (each addition is $15 \mu \mathrm{L}$ of $2.011 \mathrm{~g} / \mathrm{L}$ $\mathrm{SnO}_{2}$ ). (ii) Recovery of porphyrin emission on increasing addition of lithium perchlorate (each addition is $10 \mu \mathrm{L}$ of $1.0 \mathrm{M}$ solution) to a solution of (TMPyP) $\mathrm{Zn}: \mathrm{SnO}_{2}$ in water. (b) The picture shows fluorescence under UV irradiation of (i) (TMPyP)Zn, (ii) (TMPyP)Zn:SnO 2 in the presence of $\mathrm{LiClO}_{4}$ and (iii) (TMPyP)Zn:SnO${ }_{2}$ complex in water. 139

Figure 6.12: Fluorescence intensity of the $660 \mathrm{~nm}$ band of (TMIP)Zn on increasing addition of nanocrystalline $\mathrm{SnO}_{2}$ (dark) and lithium perchlorate (red) to a solution of (TMIP) Zn: $\mathrm{SnO}_{2}$ in water. The picture shows fluorescence under UV irradiation of (i)

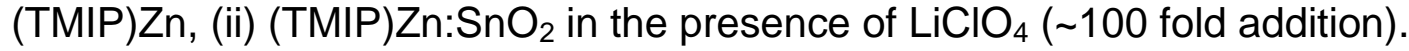
139

Figure 6.13: Corrected absorbance (background subtracted for $\mathrm{FTO} / \mathrm{SnO}_{2}$ ) spectrum of (a) zinc and (b) free-base derivatives of (i) TMPyP, (ii) TAP and (iii) TMIP derivatives electrostatically adsorbed onto the thin film $\mathrm{SnO}_{2}$ on FTO electrodes. The figure inset shows the picture of the respective $\mathrm{FTO} / \mathrm{SnO}_{2} /(\mathrm{P}) \mathrm{M}$ electrodes. 141

Figure 6.14: (a) $\mathrm{J}-V$ plots for $\mathrm{FTO} / \mathrm{SnO}_{2} /(\mathrm{P}) \mathrm{M}$ electrodes in acetonitrile containing $0.5 \mathrm{M}$ (TBA)I and $0.05 \mathrm{M} \mathrm{I}_{2}$ as redox mediator; (i) (TMPyP)Zn, (ii) (TAP)Zn, (iii) (TMPyP) $\mathrm{H}_{2}$, (iv) (TAP) $\mathrm{H}_{2}$ and (v) (TMIP) $\mathrm{H}_{2}$ surface modified. The dotted lines show the dark currents. Figure (b) shows light on-off switching of photocurrent revealing the robustness of the electrodes. The dotted line represents currents of electrode modified with only $\mathrm{SnO}_{2}$. 142

Figure 6.15: Incident photon-to-current conversion efficiency (IPCE) for $\mathrm{FTO} / \mathrm{SnO}_{2} /(\mathrm{P}) \mathrm{M}$ electrodes in acetonitrile containing (TBA) $1 / \mathrm{I}_{2}(0.5 \mathrm{M} / 0.05 \mathrm{M})$ redox mediator. The porphyrins electrostatically adsorbed are: (i) (TMPyP)Zn, (ii) (TAP)Zn, (iii) no porphyrin, (iv) $(\mathrm{TMPyP}) \mathrm{H}_{2},(\mathrm{v})(\mathrm{TAP}) \mathrm{H}_{2}$ and (vi) $(\mathrm{TMIP}) \mathrm{H}_{2}$, respectively on $\mathrm{FTO} / \mathrm{SnO}_{2}$ surface... 144 
Figure 6.16: (a) Calculated vs. measured $J_{s c}$ for different porphyrin adsorbed electrodes. Theoretical calculation were performed using the formula $J_{s c}=\int q \times F(\lambda) x$ IPCE $(\lambda) d \lambda$ where $q$ is the electron charge and $F(\lambda)$ is the incident photon flux density for AM 1.5 standard conditions at wavelength $(\lambda)$. (b) Light harvesting efficiency of (i) (TMPyP)Zn, (ii) (TMPyP) $\mathrm{H}_{2}$, (iii) (TAP) $\mathrm{H}_{2}$ and (iv) (TMIP) $\mathrm{H}_{2}$ electrodes. 145

Figure 6.17: Impedance spectra (Nyquist plots) measured at the respective $V_{O C}$ of (TMPyP)Zn and (TMPyP) $\mathrm{H}_{2}$ in dark (i and ii) and under AM1.5 light conditions (iii and iv), respectively. The figure inset shows equivalent circuit diagram used to fit the data.

Figure 7.1: (a) Absorption spectra of the compound in methanol-water solvent $\left(\mathrm{H}_{2} \mathrm{O}\right.$ $40 \% \mathrm{Vol})$. (b) Absorption spectral changes observed for ZnTMPyP $(3 \mathrm{ml}$ of $1.75 \mu \mathrm{M})$ on increasing addition $\mathrm{ZnPcS}(5-10 \mu \mathrm{L}$ of $0.489 \mathrm{mM}$ each addition) in methanol-water. (c) Mole ratio vs $\delta A$ at $430 \mathrm{~nm}$ to obtain 1:1 hetero dimer. (d) Corresponding fluorescent spectral changes observed for ZnTMPyP $(3 \mathrm{ml}$ of $\mu \mathrm{M})$ on increasing addition ZnPcS (5$10 \mu \mathrm{L}$ of $0.489 \mathrm{mM}$ each addition) in methanol-water excited at $430 \mathrm{~nm}$. The picture shows fluorescence under UV irradiation of (i) ZnTMPyP, (ii) ZnTMPyP:ZnPcS, (iii) $\mathrm{ZnPcS}$ in methanol-water solvent $\left(\mathrm{H}_{2} \mathrm{O}-40 \% \mathrm{Vol}\right)$. Figure $7.1 \mathrm{~b}$ inset shows BenesiHildebrand plot showing linear dependence of the inverse $\delta A$ at $430 \mathrm{~nm}$ on the inverse

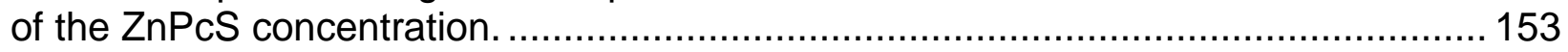

Figure 7.2: (a) Absorption spectral changes observed for $\mathrm{H}_{2}$ (TMPyP) ( $3 \mathrm{ml}$ of $1.45 \mu \mathrm{M}$ ) on increasing addition $\mathrm{Zn}(\mathrm{PcS})(5-10 \mu \mathrm{L}$ of $0.512 \mathrm{mM}$ each addition) in methanol-water. (b) Corresponding fluorescent spectral changes observed for $\mathrm{H}_{2} \mathrm{TMPyP}(3 \mathrm{ml}$ of 1.45 $\mu \mathrm{M})$ on increasing addition $\mathrm{Zn}(\mathrm{PcS})(5-10 \mu \mathrm{L}$ of $0.512 \mathrm{mM}$ each addition) in methanolwater excited at $438 \mathrm{~nm}$ Figure 1a inset shows Benesi-Hildebrand plot showing linear dependence of the inverse $\delta A$ at $438 \mathrm{~nm}$ on the inverse of the $\mathrm{Zn}(\operatorname{PcS})$ concentration. 154

Figure 7.3: (a) Absorption spectral changes observed for $\mathrm{H}_{2}$ TMPyP $(3 \mathrm{ml}$ of $1.45 \mu \mathrm{M}$ ) on increasing addition $\mathrm{H}_{2}(\mathrm{PcS})(5-10 \mu \mathrm{L}$ of $0.551 \mathrm{mM}$ each addition) in methanol-water. (b) Corresponding fluorescent spectral changes observed for $\mathrm{H}_{2}(\mathrm{TMPyP})$ ( $3 \mathrm{ml}$ of 1.45 $\mu \mathrm{M})$ on increasing addition $\mathrm{H}_{2}(\mathrm{PcS})(5-10 \mu \mathrm{L}$ of $0.551 \mathrm{mM}$ each addition) in methanolwater excited at $438 \mathrm{~nm}$ Figure 1a inset shows Benesi-Hildebrand plot showing linear dependence of the inverse $\delta \mathrm{A}$ at $423 \mathrm{~nm}$ on the inverse of the $\mathrm{H}_{2}(\mathrm{PcS})$ concentration. 155

Figure 7.4: (a) Corrected normalized absorbance (background subtracted for FTO/SnO ${ }_{2}$ ) spectrum of (i) $\mathrm{ZnPcS} / \mathrm{SnO}_{2}$ (ii) $\mathrm{ZnTMPyP}: \mathrm{ZnPcS} / \mathrm{SnO}_{2}$ and (iii) $\mathrm{H}_{2} \mathrm{TMPyP}$ : $\mathrm{ZnPcS} / \mathrm{SnO}_{2}$. The Figure inset shows the picture of the respective electrodes. (b) shows light on-off switching of photocurrent electrodes i) $\mathrm{ZnPcS} / \mathrm{SnO}_{2}$ (ii) $\mathrm{ZnTMPyP}$ : $\mathrm{ZnPcS} / \mathrm{SnO}_{2}$ and(iii) $\mathrm{ZnTMPyP} / \mathrm{SnO}_{2}$. (c) Incident photon-to-current conversion efficiency (IPCE) for (i) $\mathrm{ZnPcS} / \mathrm{SnO}_{2}$ (ii) $\mathrm{ZnTMPyP}: \mathrm{ZnPcS} / \mathrm{SnO}_{2}$ and (iii) $\mathrm{H}_{2} \mathrm{TMPyP}$ : $\mathrm{ZnPcS} / \mathrm{SnO}_{2}$. (d) IPCE for (iv) $\mathrm{H}_{2} \mathrm{PcS} / \mathrm{SnO}_{2}$ (v) $\mathrm{ZnTMPyP}: \mathrm{H}_{2} \mathrm{PcS} / \mathrm{SnO}_{2}$ and (vi) 
$\mathrm{H}_{2}$ TMPyP: $\mathrm{H}_{2} \mathrm{PcS} / \mathrm{SnO}_{2}$. All photoelectrochemical experiments were performed in acetonitrile containing $0.5 \mathrm{M}(\mathrm{TBA}) \mathrm{I}$ and $0.05 \mathrm{M} \mathrm{I}_{2}$ as redox mediator. 157

Figure 7.5: (a) $\mathrm{J}-\mathrm{V}$ plots of $\mathrm{ZnPcS} / \mathrm{SnO}_{2}$ and $\mathrm{ZnTMPyP}: \mathrm{ZnPcS} / \mathrm{SnO}_{2}$ electrodes in dark (i and ii) and under AM1.5 light conditions (iii and iv), respectively.(b) $J-V$ plots of (iii) $\mathrm{SnO}_{2} / \mathrm{H}_{2}$ (PcS) (i), $\mathrm{SnO}_{2} / \mathrm{Zn}(\mathrm{PcS}): \mathrm{H}_{2}$ (TMPyP) (ii), $\mathrm{SnO}_{2} / \mathrm{H}_{2}(\mathrm{PcS}): \mathrm{H}_{2}$ (TMPyP) (iii), and $\mathrm{SnO}_{2} / \mathrm{H}_{2}(\mathrm{PcS}): \mathrm{Zn}(\mathrm{TMPyP})$ under AM1.5 light conditions. The electrolyte used in this experiment consists of $0.5 \mathrm{M}(\mathrm{TBA}) \mathrm{I}$ and $0.05 \mathrm{M} \mathrm{I}_{2}$ as redox mediator....................... 158

Figure 7.6: Impedance spectra (Nyquist plots) measured at the respective $V_{o c}$ of $\mathrm{ZnPcS} / \mathrm{SnO}_{2}$ and $\mathrm{ZnTMPyP}: \mathrm{ZnPcS} / \mathrm{SnO}_{2}$ in dark (i and ii) and under AM1.5 light conditions (iii and iv), respectively. The Figure inset shows equivalent circuit diagram used to fit the data. 


\section{CHAPTER 1}

\section{INTRODUCTION}

\subsection{Introduction}

Supramolecular chemistry is the chemistry of non-covalent molecular bonds and it involves interactions between the two classes of compounds: host and guest molecules ${ }^{1}$. A host molecule binds the guest molecule to form a host-guest complex or a supramolecule. Hence it is also known as host-guest chemistry. The interactions within the supramolecules are based on two important concepts: complementarity and preorganization $^{1}$. Complementarity indicates that a host molecule should have binding sites with appropriate electronic character to complement the guest molecules. A host molecule is said to be preorganised if there are no significant conformational changes upon binding the guest molecule. Supramolecular chemistry deals with non-covalent interactions involving attractive and repulsive force in different forms.

Non-covalent interactions are classified based on the intermolecular forces as shown in the Figure 1.1. Designing a specific application based on non-covalent interactions requires interplay of more than one type of listed interactions. Applications of multiple-interaction-based supramolecular systems are already known in nature and they are part of our life. A few examples are: self-assembly of photosensitizer molecules in plant photosynthesis, uptake and transport of oxygen by haemoglobin, helical structure of DNA and various neurotransmitter hormones ${ }^{1}$. Understanding and mimicking the systems in nature have led to the development and utilization of new concepts to solve the critical issues. Currently, supramolecular chemistry is being 
explored for wider applications such as ion sensing, photodynamic therapy, molecular wires, rectifiers, logic gates, switches and catalysis ${ }^{1}$.

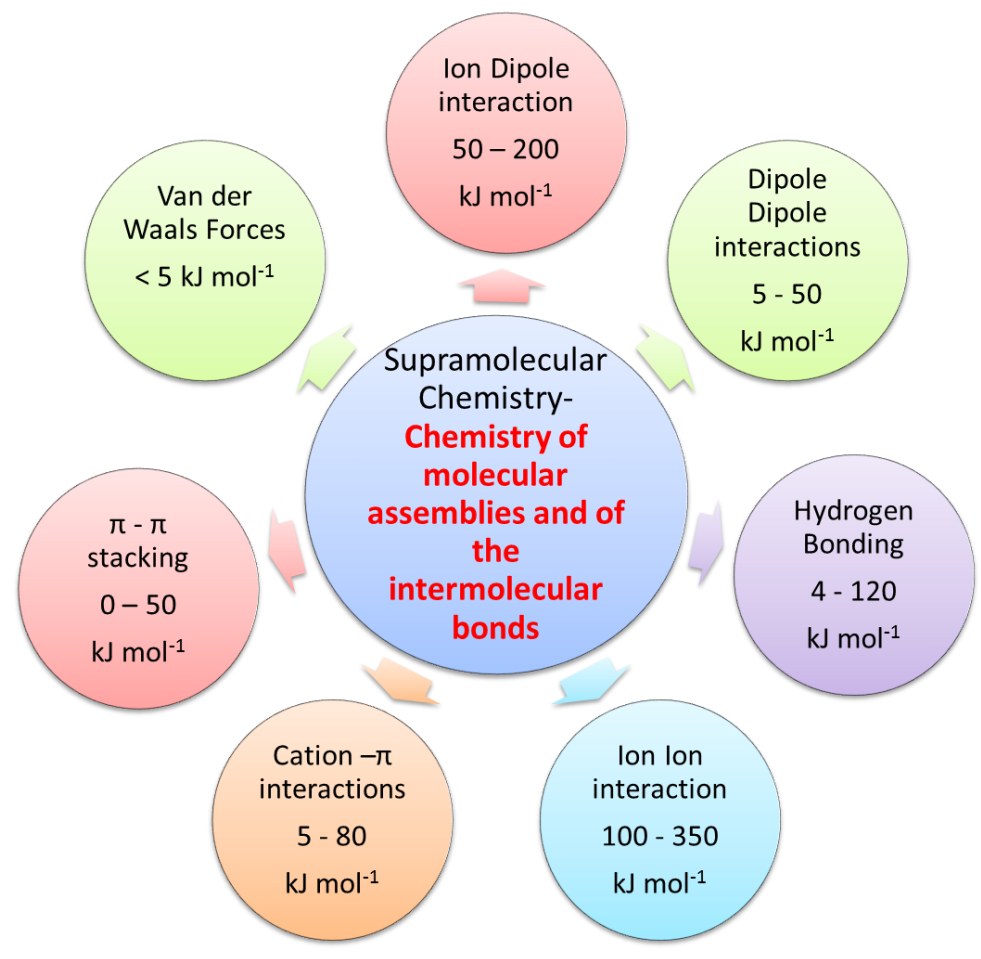

Figure 1.1: Classification of supramolecular interactions.

\subsection{Biosupramolecular Photosynthetic System}

Building novel molecular systems that can efficiently convert solar energy into fuels such as hydrogen is currently being studied due to various reasons: increase in the energy consumption due to industrial globalization, decrease in the fossil fuel reserves and also increased concern about the environment. In nature, the supramolecular system that converts solar energy to chemical energy is called the photosynthetic system. In this system, there are self-assembled dyes or pigment molecules in a protein matrix that can absorb wavelengths throughout the visible region of light. Their function is to absorb the sunlight and funnel the energy to the reaction center. This phenomenon is called energy transfer, occurs only if the emission of one 
dye (energy donor) overlaps with absorption of another dye (energy acceptor). Such emission overlapped dyes are required to be positioned in a specific orientation as well as arranged in a particular distance to ensure efficient energy transfer occurrence. Nature has already taken care of such factors utilizing supramolecular chemistry intelligently to perform the cascading event (energy transfer). Sequential energy transfer event in Porphyridium cruetum and the corresponding absorption is shown in Figure $1.2 \mathrm{~b}$ and Figure 1.2a respectively ${ }^{1}$.

(a)

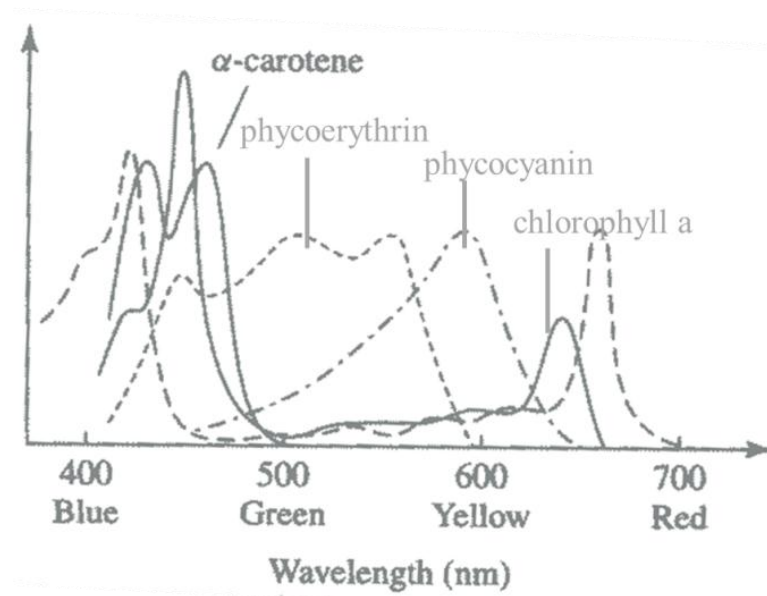

(b)

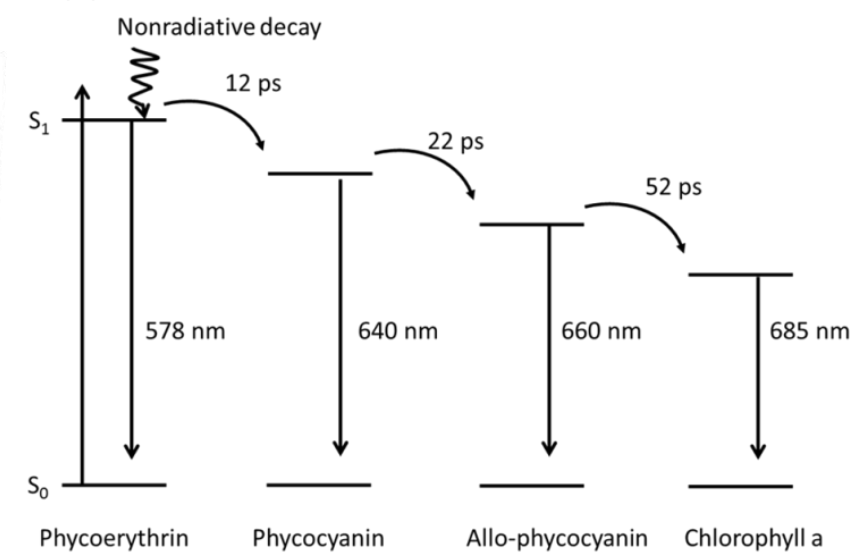

Figure 1.2: a) Absorption spectra of various energy capturing pigments from algae and plants. b) Energy-transfer cascade for antenna pigments in light-harvesting complexes of the algae Porphyridium cruetum.

In the photosynthetic reaction center, light induced multiple electron transfer processes result in the formation of long lived charge separated states. For example, in photosynthetic bacterium Rhodopseudomonas viridis, the photoinduced electron transfer (PET) process is initiated at the reaction center upon excitation of bacteriochlorophyll dimer $\left(D_{\mathrm{L}}\right)$ also known as $\mathrm{P}_{960}{ }^{2,3}$. Following $\mathrm{PET}$, a sequential electron transfer to bacteriochlorophyll a monomer $\left(B_{A}\right)$ and then to bacteriopheophytin $\left(\phi_{\mathrm{A}}\right)$ occurs as shown in Figure 1.3. Bacteriopheophytin is structurally similar to 
chlorophyll monomer but without metal atom $\left(\mathrm{Mg}^{+2}\right.$ ion) at the center - making it easier to reduce (lower reduction potential) thereby facilitating facile electron transfer event $(\mathrm{PET})^{3}$. From the reduced bacteriopheophytin, the electrons are being transferred to para-quinone $\left(Q_{A}\right)$ and then to meta-quinone $\left(Q_{B}\right)$, resulting in a long-lived charge separated state $D_{L}{ }^{+}-Q_{A}{ }^{-*}$ with a life time of about $1 s^{3}$. In smaller organisms, the positively charged cation is slowly regenerated through haeme centres of cytochrome proteins. In more complex systems it serves as a substrate for sequential hole (positive charge) transfer including manganese containing oxidation catalyst for water oxidation. Thus favorable energetics and orientation are needed for this type of vectorial photoinduced electron transfer to take place.



Figure 1.3: Charge separation at the photosynthetic reaction centre of $\underline{R}$. viridis. 
It is worth considering Bacteriochlorophyll-a as a supramolecule which has already complementary and preorganised factors to get self-assembled in the photosynthetic membrane. Bacteriochlorophyll a has a long aliphatic chain attached to a magnesium-containing tetrapyrrole macrocycle. The purpose of aliphatic chain is to anchor the pigment in the phospholipid photosynthetic membrane. It also contains two partially-hydrogenated pyrrole rings to extend its absorption to longer wavelength. Magnesium metal atom at the center plays a pivotal role in arrangement. Two axial coordinating sites of magnesium $\left(\mathrm{Mg}^{+2}\right)$ ion are coordinated to the polypeptide side chain ligand to increase the stability and control the orientation. Similar kind of axial coordination are found in other naturally-occurring metal atom containing tetra pyrrole complexes such as iron ( $\mathrm{Fe})$ in haemoglobin for oxygen-binding and cobalt (Co) in Coenzyme $B_{12}{ }^{1}$. So the structure and phenomenon occurring in natural systems serve as a protocol for designing and developing efficient energy harvesting and storage devices for the future.

1.3 Photoinduced Electron Transfer (PET) and Photoelectrochemical Cells (PECs)

\subsubsection{Photoinduced Electron Transfer (PET)}

Light assisted electron transfer reactions are called photoinduced electron transfer reactions. PET is the quenching of the emission of excited chromophore due to electron transfer in the presence of another molecule called a quencher. Depending on the type of quencher two types of PET are listed below ${ }^{4}$ as shown in the Figure 1.4.

In oxidative quenching the excited electrons of the chromophore are being transferred from lowest unoccupied molecular orbital (LUMO) to the LUMO of the quencher resulting in an oxidized chromophore and a reduced quencher. This type of 
quenching is possible only if the LUMO of the quencher is lower in energy compared to LUMO of the chromophore i.e. if the quencher is an acceptor (see Figure 1.4a).

If the quencher acts an electron donor, then second type of quenching called reductive quenching occurs. Upon the chromophore excitation, the electrons from the quencher $(\mathrm{HOMO})$ are quickly transferred to the $\mathrm{HOMO}$ of the chromophore resulting in an oxidized quencher and a reduced chromophore as shown in the Figure 1.4b. The electron transfer rate from the quencher to chromophore should be higher than the rate of relaxation of electrons from the excited state to the ground state of the chromophore. Depending on the selection of appropriate chromophore and quencher, the directionality in the electron transfer could be easily defined and used for construction of energy harvesting devices. Hence it is very important to understand the mechanisms of PET before designing specific energy conversion devices. Various factors that are need to be considered for designing the donor- acceptor systems based on PET are listed below ${ }^{5}$.

- Absorption and emission of the electron donor and acceptor molecules

- Redox potential of the donor and acceptor's molecules

- Excited state redox potentials i.e. negative free energy for electron transfer from the excited state of the donor to the acceptor

- Suitable orientation and distance between the chromophore to enable faster charge separation and slower recombination

- Solvent reorganization energy 
The free energy change for the electron transfer quenching reaction can be calculated by using Rehm-Weller equation. ${ }^{6,7}$ The driving force for charge recombination $\left(-\Delta G_{c r}\right)$ and charge separation $\left(-\Delta G_{c s}\right)$ are calculated according the following equations.

$$
\begin{gathered}
-\Delta G_{C R}=E_{O X}-E_{R E D}-\Delta G_{S} \\
-\Delta G_{C S}=\Delta E_{0-0}-E_{O X}+E_{R E D}+\Delta G_{S}
\end{gathered}
$$

Where $E_{o x}$ is the first oxidation potential of the donor, $E_{r e d}$ are the first reduction potential of the acceptor, $\Delta E_{0-0}$ is the energy of the $0-0$ transition between the lowest excited state and the ground state of the donor evaluated from the spectral studies, $\Delta G_{s}$ is static energy calculated by using the " dielectric continuum model"

$$
\Delta G_{S}=\frac{e^{2}}{4 \pi \varepsilon_{0} \varepsilon_{R} R_{C C}}
$$

where $\varepsilon_{0}$ and $\varepsilon_{s}$ represents vacuum permittivity and dielectric constant of the solvents respectively, $R_{\mathrm{cc}}$ is the value of the center-to-center distance between the donor and acceptor. The ideal system requires a substantial driving force for charge separation process (more negative $\Delta G_{c s}$ ) and lower energy for charge recombination process to ensure long lived charged separated states.

With detailed and systematic study on PET events using donor - acceptor dyads and establishing long-lived charged separated states; the main objective of studying PET is to generate fuels such as hydrogen, methane and methanol using solar energy ${ }^{8}$. Devices that are used for this purpose are called photoelectrochemical cells. 


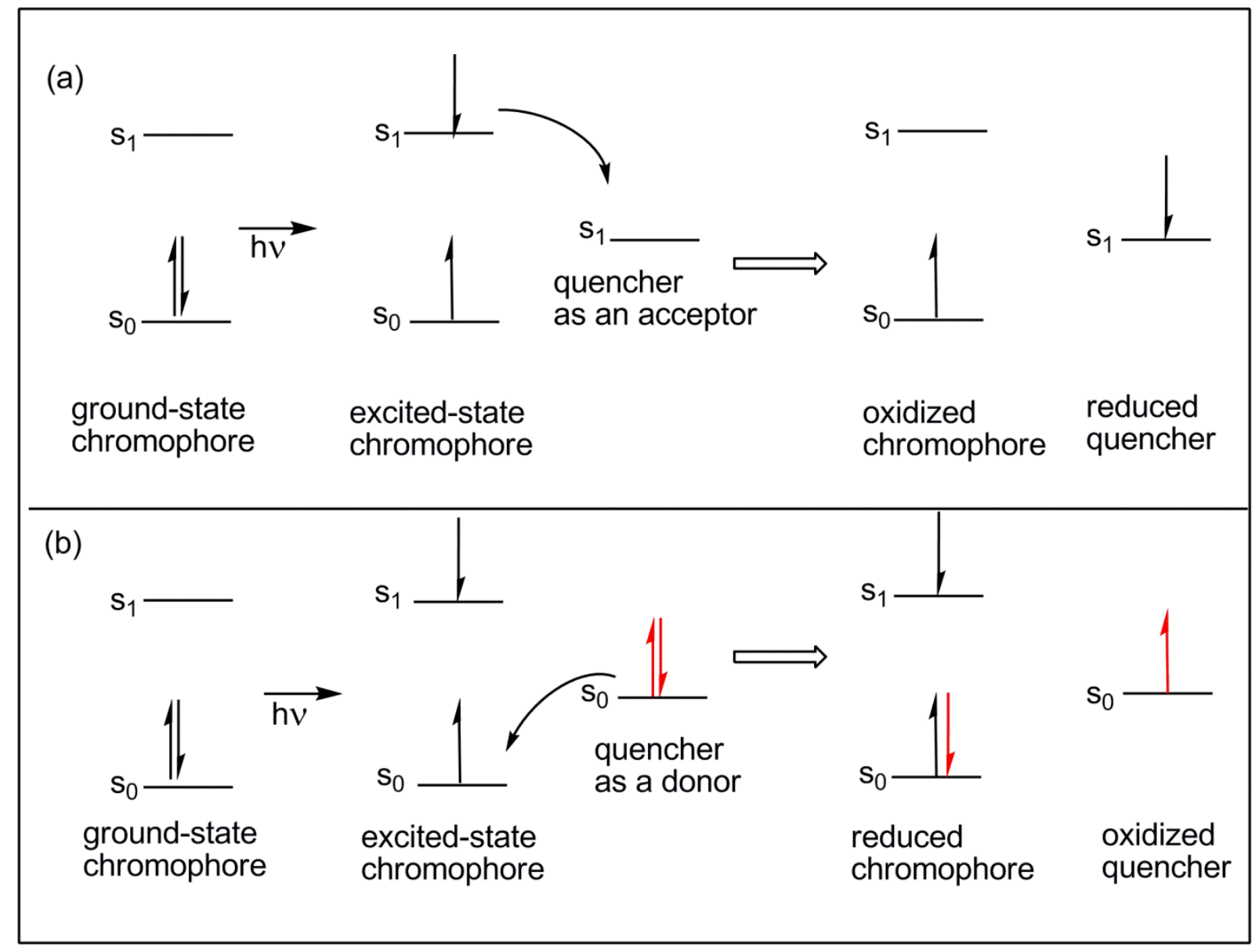

Figure 1.4: Mechanism of a) oxidative quenching b) reductive quenching. $S_{0}$ and $S_{1}$ are HOMOs and LUMOs respectively.

\subsubsection{Photoelectrochemical Cells (PECs)}

Photoelectrochemical cells are the electrochemical devices that can be used to convert light into current in presence of the redox mediating electrolyte. These cells are made up of a semiconductor-based working electrode, redox electrolyte and a counter electrode. PECs are classified into 3 types of cells based on the type of electrolyte ${ }^{9}$ as shown in Figure 1.5.

1. Photovoltaic cells

2. Photoelectrosynthetic cells

3. Photocatalytic cells 
In photovoltaic cells, the reaction at the counter electrode is in reverse of the photoredox reaction at the semiconductor working electrode. The redox electrolyte acts as mediator shuttling electrons between the two electrodes through ions, resulting in no net change in the solution composition and materials. These types of cells are mainly used to generate photocurrent from sunlight. Direction of electron flow is governed by the type of semiconductor that is being used. In n-type semiconductors, excited electrons move towards the electrode resulting in photoanodic current. In p-type semiconductors, the excited electrons travel towards the electrolyte and generating photocathodic current.

In photoelectrosynthetic cells, two different redox reactions occur at the semiconductor working electrode and counter electrode. As a result, the light energy is converted into chemical energy in these cells. Unlike photovoltaic cells, the redox electrolytes are different for each electrode and so a separator is usually employed. The position of the redox potential of the electrolyte with respect to conduction or valence bands of corresponding semiconductor is very crucial. For example, the oxidation potential of the electrolyte on the n-type semiconductor side should be above the valence band edge. Photocatalytic cells are similar to photoelectrosynthetic cells except the locations of the redox potentials are changed. 


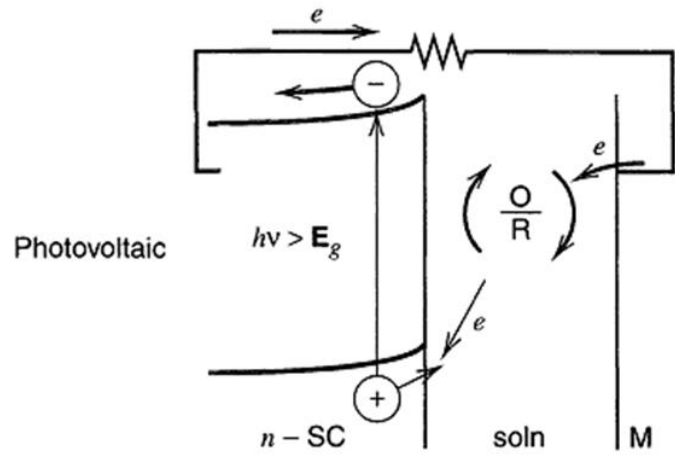

(a)

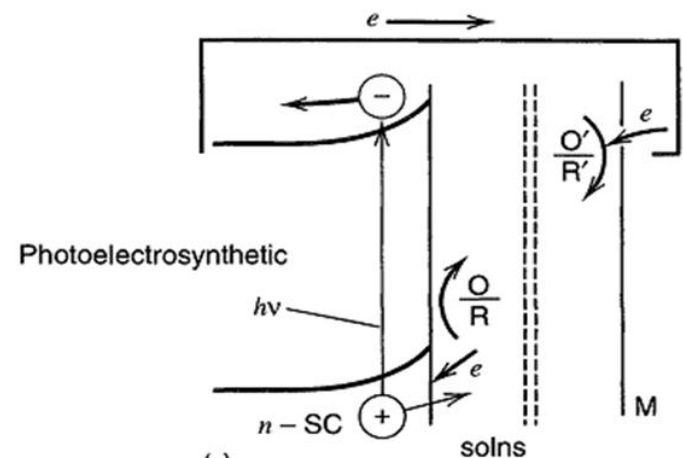

(c)

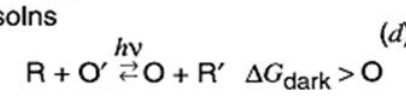

$(d)$ $(e)$

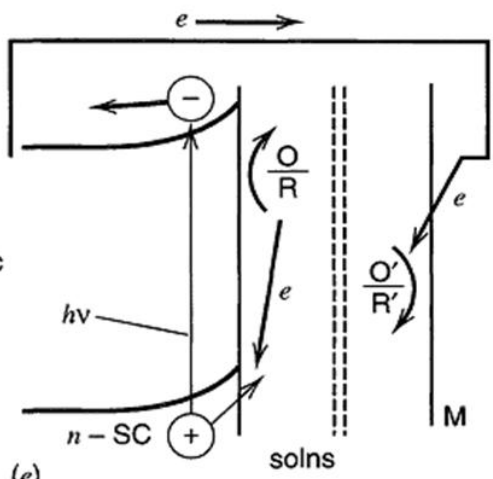

$$
\mathrm{R}+\mathrm{O}^{\prime} \stackrel{h v}{\rightleftarrows \mathrm{O}}+\mathrm{R}^{\prime} \quad \Delta G_{\text {dark }}<\mathrm{O}
$$
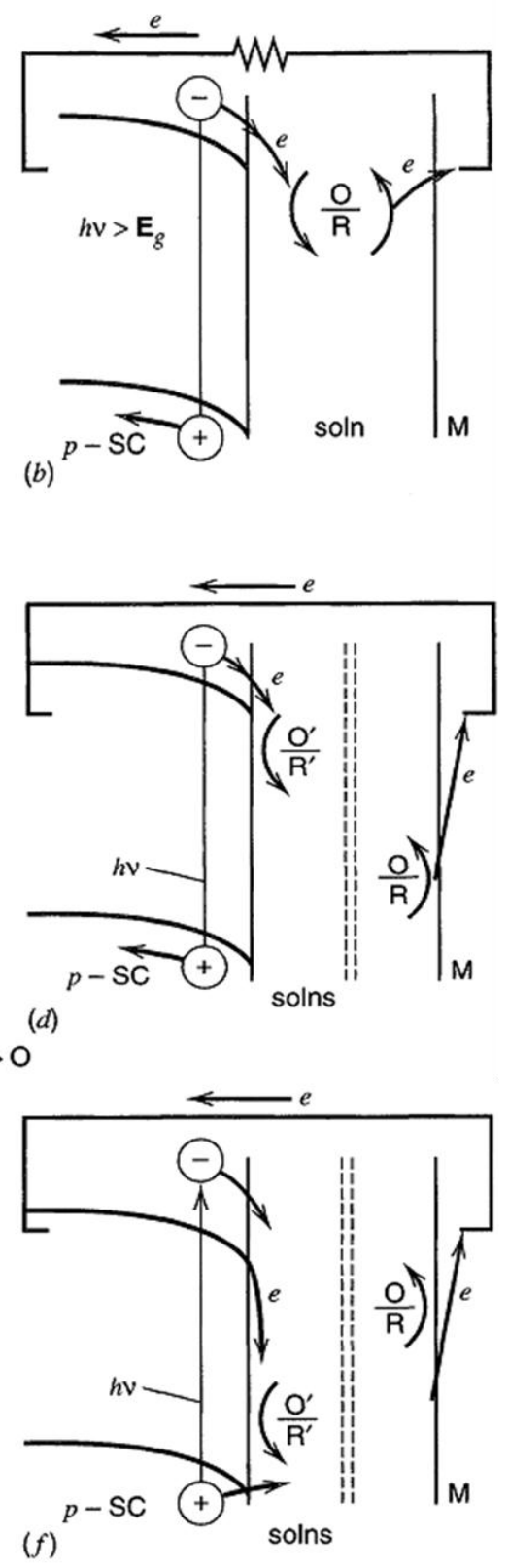

Figure 1.5: Types of photoelectrochemical cells( ntype, ptype) (a, b) photovoltaic cells $(c, d)$ photoelectrosynthetic cells $(d, e)$ photocatalytic cells ${ }^{9}$.

Examples for all 3 types of photoelectrochemical cells are shown in the Figure 1.5. In all 3 cases, the usage of semiconductors for photoredox reactions is limited by the corresponding position of band gap. The energy of the light needed for photoexcitation should be higher than the band gap of the semiconductors of the Photo 
Electrochemical Cells (PECs). For example, a $3 \mathrm{eV}$ band gap semiconductor such as n-type titanium dioxide $\left(\mathrm{TiO}_{2}\right)$ gets excited only with energy higher than $3 \mathrm{eV}$ corresponding to the UV portion of the radiation. Since the energy of sunlight in reaching the earth surface $(400-800 \mathrm{~nm})$ is lower than the band gap, sunlight is not useful for initiating photoredox reaction. A better approach in order to employ $\mathrm{TiO}_{2}$ for capturing longer wavelength energy involves dye sensitization of the semiconductor ${ }^{10}$. Here the dyes that are sensitized on $\mathrm{TiO}_{2}$ utilize the energy of sunlight and inject the electrons into the conduction band generating photocurrent. By controlling the position of excited states of the dye with respect to the conduction band of $\mathrm{TiO}_{2}$, the direction of electron flow could be controlled. Thus, it is very important to design a unidirectional electron movement in photoelectrochemical cells for efficient energy harvesting. Here we are interested in the photovoltaic cells for solar cell applications and the sequence of electron-transfer steps is explained using a photosensitizer $(\mathrm{S})$ absorbed on $\mathrm{TiO}_{2}$ with a redox mediator $(\mathrm{O} / \mathrm{R})$ and a counter electrode as shown in the Figure $1.6^{4}$.

At the anode the dye molecule (S) absorbs the light leading to the formation of excited dye molecule $\left(S^{*}\right)$. In other words, electrons are transferred from the ground state to the excited state, resulting in photoexcitation.

$$
S+h v \rightarrow S^{*} \text { (photoexcitation) }
$$

Excited electrons of the dye molecule are either injected into the conduction band of $\mathrm{TiO}_{2}$ or it could relax back to the ground state resulting in emission.

$$
\begin{gathered}
S^{*} \rightarrow S+h v^{\prime}(\text { emission }) \\
S^{*} \rightarrow S^{+}+e-c b\left(\mathrm{TiO}_{2} \text { Charge injection }\right)
\end{gathered}
$$




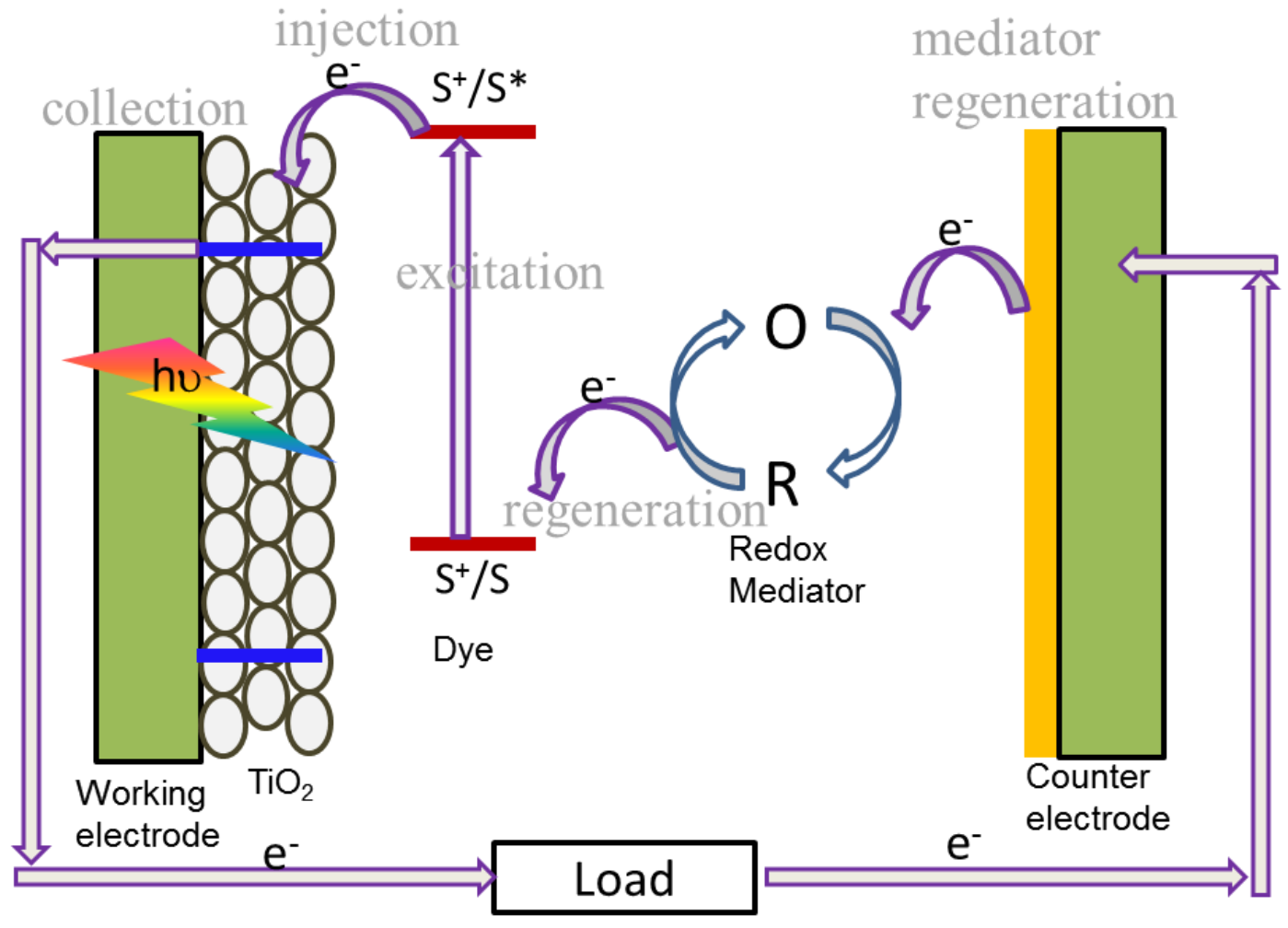

Figure 1.6: Schematic diagram of the photoelectrochemical cell composed of dye (S) sensitized $\mathrm{TiO}_{2}$ semiconductor (n-type), redox mediator $\left(O \rightleftharpoons R+e^{-}\right)$and counter electrode.

While the injected electrons passes through the $\mathrm{TiO}_{2}$ and reach the current collector, the oxidized dye molecule is regenerated or reduced quickly by the donor $(\mathrm{R})$ of the redox mediator. Unfavorable events, such as recombination of electrons from the $\mathrm{TiO}_{2}$ to the oxidized dye molecule, result in low photocurrent generation.

$$
S^{+}+R \rightarrow S+O(\text { dye regeneration) }
$$

The oxidized species $(\mathrm{O})$ of the the mediator then gets reduced at the counter electrode with electron from the external circuit. Thus sunlight acts as the source of energy to activate the flow of electrons leaving no net change in the overall process.

$$
O+e^{-} \rightarrow R \text { (regenertation of the mediator) }
$$




\subsubsection{Key Parameter of PECs}

Irrespective of the kind of photoelectrochemical cells, the electrochemical characterization parameters remain the same. Important characterization parameters measured under dark and light conditions are listed below ${ }^{4}$.

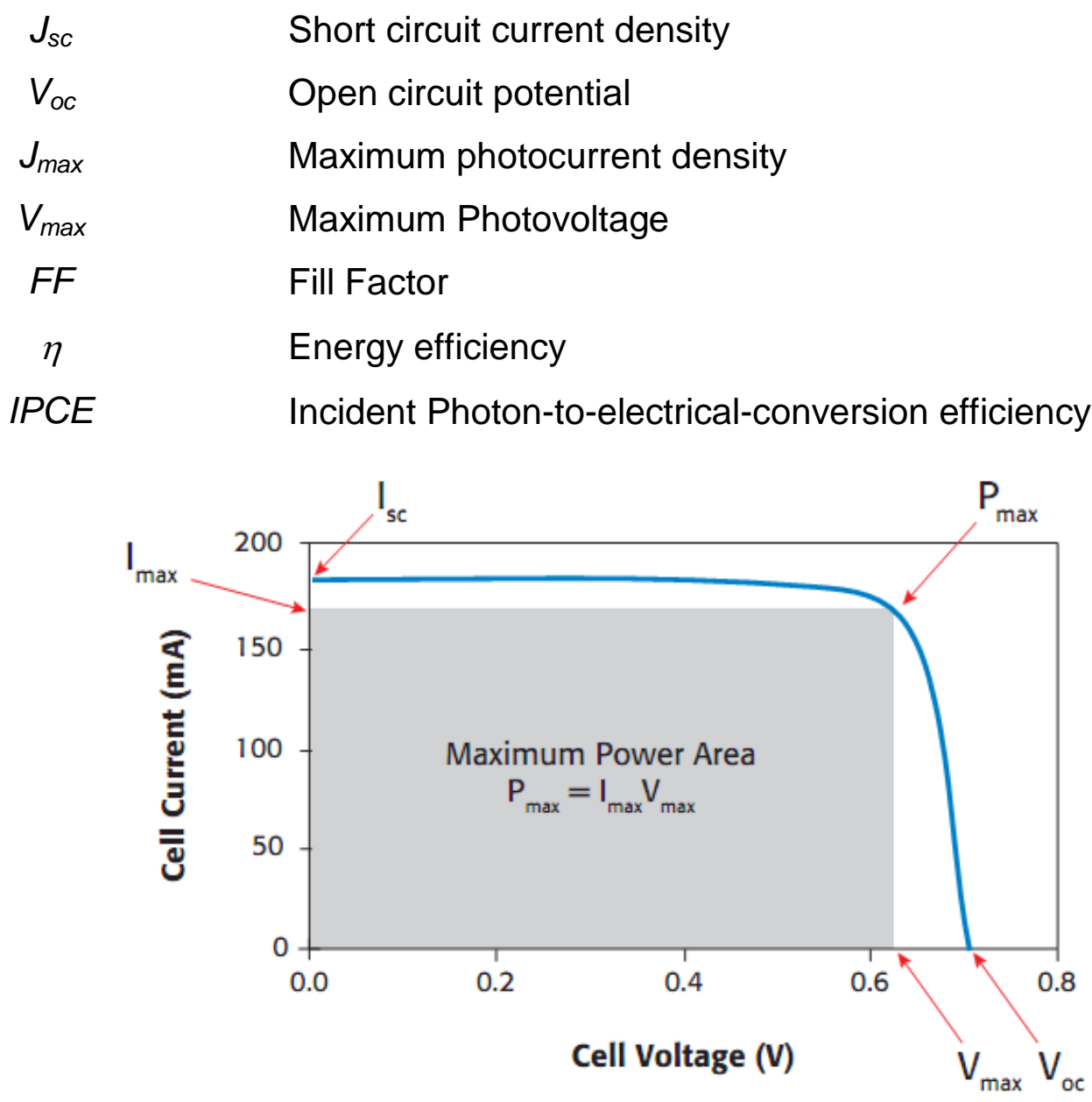

Figure 1.7: Model $I-V$ curve of the photoelectrochemical cell.

The overall sunlight-to-electric-power conversion efficiency or energy efficiency is calculated as shown in the equation. It is also quantifies the amount of useful energy that could be extracted from the given photocells. Typical $I-V$ curve and the parameters are shown in the Figure 1.7. 


$$
\eta=\frac{P_{\max }}{P_{\text {in }}}=\frac{J_{S c} \cdot V_{o c} \cdot F F}{P_{i n}}
$$

IPCE is the measure of adsorbed dye/metal oxide system's efficiency to inject photo current generating electrons under monochromatic light or individual wavelength of light. This is also called quantum efficiency. The IPCE spectrum is known as photoaction spectra. IPCE $(\lambda)$ can be calculated as shown in the equation.

$$
\operatorname{IPCE}(\lambda)(\%)=1240\left(\frac{J_{S C}}{\lambda * P_{\text {in }}}\right) * 100
$$

$\lambda$ is the monochromatic incident light wavelength $(\mathrm{nm}), P_{\text {in }}$ is the power density of the incident light intensity at that wavelength $\left(\mathrm{mW} / \mathrm{cm}^{2}\right)$ and $J_{s c}\left(\mathrm{~mA} / \mathrm{cm}^{2}\right)$ is the short circuit current density at that particular wavelength $(\lambda)$. IPCE of the dye sensitized metal oxide system is divided into three terms as shown below ${ }^{4}$.

$$
I P C E=\eta_{a b s} \cdot \eta_{i n j} \cdot \eta_{\text {coll }}
$$

Where $\eta_{a b s}$ is efficiency of the light absorption by the dye or called light harvesting efficiency; $\eta_{i n j}$ is the injection efficiency dealing with electrons injected from the excited state of the dye; and $\eta_{\text {coll }}$ is the charge collection efficiency dealing with the charge transport property of the semiconductor layer. An ideal dye molecule in dye sensitized systems would be the one which absorbs the sunlight in the visible and near IR region. The dye system could be one single molecule or composed of more than one dye based composite system. It is very important to establish good electronic coupling between electron accepting level of conduction band of the metal oxide and the lowest unoccupied molecular orbital (LUMO) of the dye molecule. The charge injection 
efficiency depends on the electronic interactions between the excited state of the dye molecule and the conduction-band edge of the semiconductor.

\subsection{PECs Based on Artificial Photosynthesis}

\subsubsection{Donor and Acceptor Molecules}

To mimic natural photosynthesis, several covalently and non-covalently linked light harvesting systems have been investigated in solutions till now ${ }^{11-16}$. Frequently used donor and acceptor molecules are listed below.

Donor

Porphyrin, Phthalocyanine, Ferrocene,

Tetrathiafulvalene, Oligo-(poly)-

phenylene vinylenes, Naphthalocyanine,

BODIPY,Oligothiophenes
Acceptor

Quinones, Fullerenes,

Perylenecarboxiimides,

Tetracyanoethylenes

To understand the covalent and non-covalent bio-mimicking systems, two donoracceptor models are discussed below.

A classical covalently linked donor- acceptor based triad system was (Fc-ZnP$\left.\mathrm{C}_{60}\right)$ designed by Imahori and coworkers ${ }^{17}$. It involves zinc porphyrin $(\mathrm{ZnP})$ as primary donor, fullerene $\left(\mathrm{C}_{60}\right)$ as acceptor and ferrocene $(\mathrm{Fc})$ as secondary donor as shown in the Figure 1.8a. In benzonitrile, upon excitation of porphyrin, PET occurs from both singlet and triplet state resulting fullerene anion radical and porphyrin cation radical (Fc-

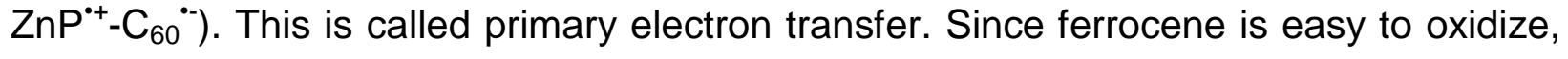
secondary electron transfer occurs resulting in the charge separated states of ferrocene

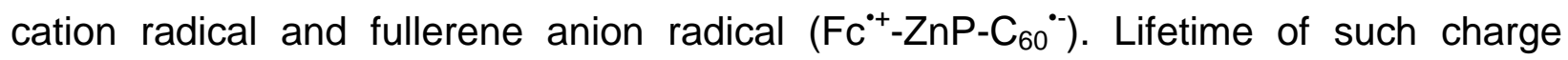
separated state for this triad was found to be $16 \mu \mathrm{s}$. In covalent linked system, there is a 
necessity for fairly intensive design resulting in complex synthesis. At the same time, there is no need to concern about other competing events.

(a)

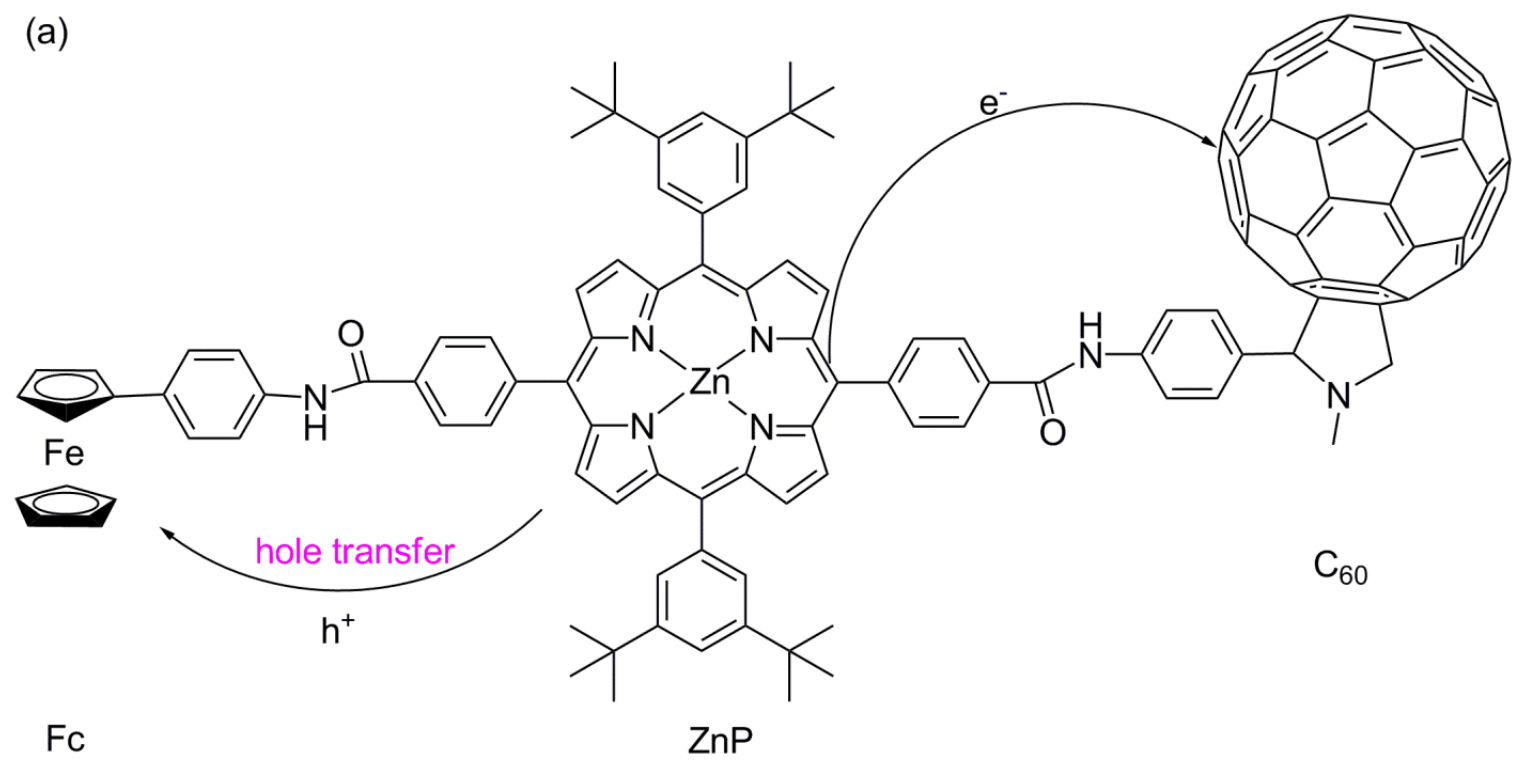

(b)

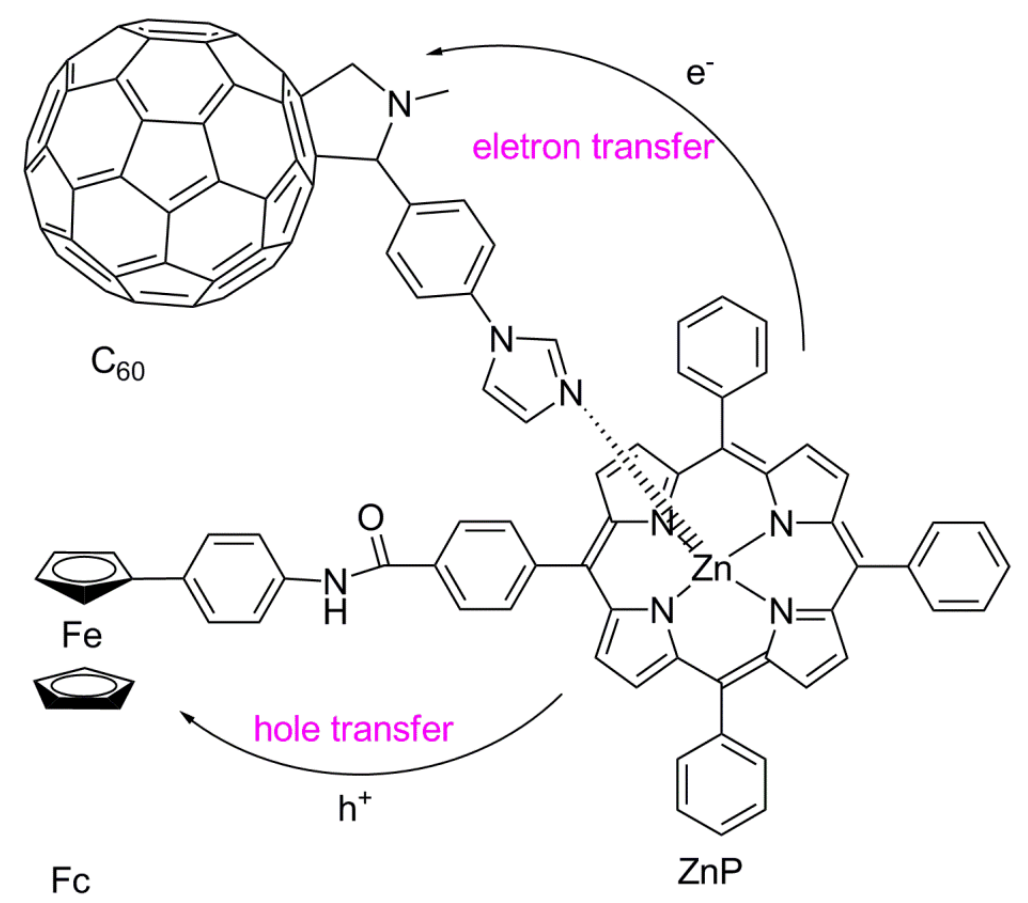

Figure 1.8: a) Covalently linked donor-acceptor triad b) Non-covalently linked donoracceptor dyad.

Supramolecular system with the same combination of $\mathrm{ZnP}, \mathrm{C}_{60}$ and $\mathrm{Fc}$ was reported by D'Souza and his coworkers. ${ }^{18}$ They reported the axial coordination between 
fullerene and zinc porphyrin - ferrocene dyad resulting in supramolecular triad as shown in Figure 1.8b. It is very clear that orientation of $\mathrm{C}_{60}$ in non-covalent system is different from that of the covalent linked triad. Upon excitation of porphyrin both primary and secondary electron transfer event occurs resulting in ferrocene cation and fullerene anion. The lifetime of the resulting similar charge separated states was smaller than covalently linked system due to faster electron recombination. The reported reason was closer orientation and interaction between the fullerene and the ferrocene. Thus with supramolecular systems, it is very necessary to consider multiple interaction possibilities between the host - guest complexes and other competing events. For example usage of the polar solvents, such as methanol, acetone should be prohibited as they interfere with axial coordination. However, the non-covalent systems provides room for easy expansion of the system with different acceptors molecules unlike covalent. ${ }^{13}$

To design PECs using the covalent and non-covalent donor-acceptor system, it is very important to orient them in a particular fashion to promote the unidirectional electron movement. Such carefully designed PECs are systematically discussed here.

\subsubsection{PECs Based on Covalent Systems}

Various techniques such as, Langmuir-Blodgett (LB) films, self-assembled monolayer on $\mathrm{Au}$ and Ito (SAM), electrophoretic deposition have been employed for forming donor-acceptor based PECs. Using an appropriate model system as an example the technique, design and the performance of the cells are explained in this section. 


\subsubsection{Langmuir-Blodgett (LB) Films}

In this technique amphiphilic donor - acceptor molecules in volatile organic solvents are spread evenly on an aqueous phase to form packed monolayer film on the surface after evaporation of organic solvent. Monolayer is formed such that hydrophilic layer point toward the aqueous layer and lipophilic layer point up into the gas/air phase ${ }^{1}$. Such monomolecular thick layer is formed using Langmuir trough and the layers could be easily transferred on to various material surfaces such as metal oxides, silicon, metals etc. Multilayer formation on the substrate could be controlled by repeating the film formation process. In order to build PECs, the donor and acceptor molecules are introduced into the monolayer using appropriate amphiphiles. It is found that fullerenes functionalized with polar atom such as nitrogen or oxygen could form stable Langmuir films and also showed improved transforming ability. Hence, Chunhui and his coworkers formed a monolayer fullerene films on the tin oxide surface ${ }^{19}$. They have used long alkyl chain such as arachidic acid and functionalized fullerene for this purpose. Based on their calculation it was shown that fullerenes were packed in between the arachidic acid groups due to strong hydrophobic interactions. PECs tested for fullerene modified electrode using nitrogen purged ascorbic acid as electrolyte showed upto 1.6 uA under $0.1 \mathrm{~V}$ (Figure 1.9a). Later Tommi ${ }^{20}$ and coworker formed LB bilayer film using a covalently linked porphyrin-fullerene dyad and regioregular poly-3-hexylthiophene (PHT) (Figure 1.9b). They found PET from porphyrin to fullerene followed by electron transfer from PHT to porphyrin resulted in photocurrent generation. For molecular level characterization of donor-acceptor films ${ }^{19-25}$, LB technique is suitable, however unfit for construction of large scale devices. 
(a)

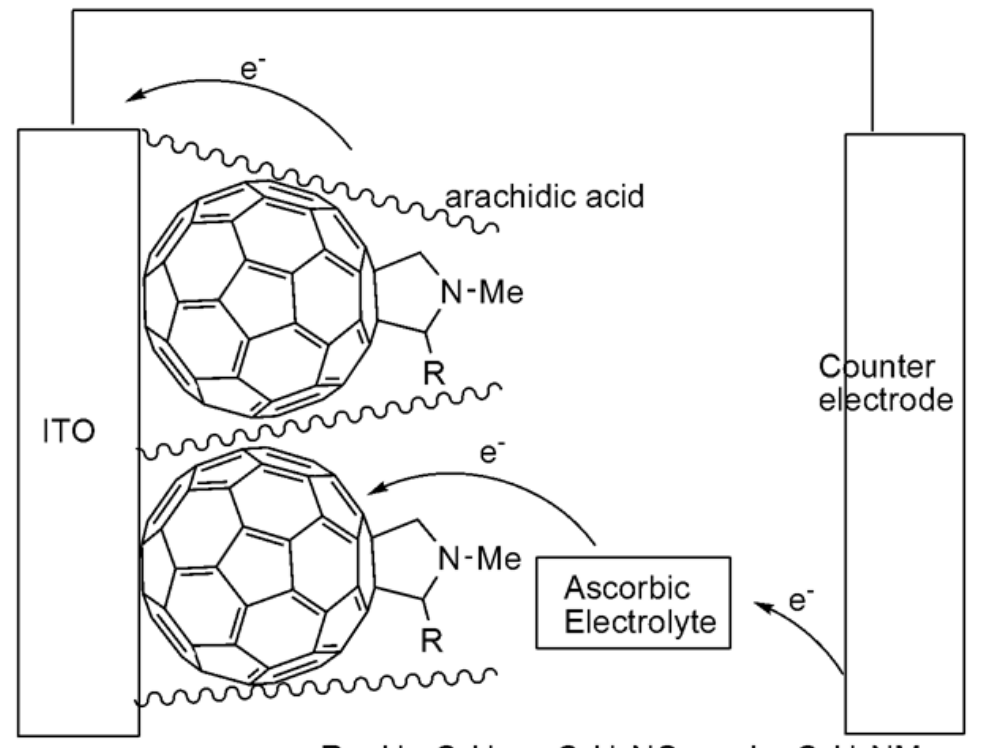

(b)



Figure 1.9: a) Photoelectrochemical cell based on LB film using fullerene derviatives and arachidic acid. b) Porphyrin fullerene dyad and Poly-Hexyl Thiophene (PHT) used for the formation of LB film.

\subsubsection{Self- Assembled Monolayer Films (SAMs)}

Commonly, SAMs are formed by chemisorption of thiol $(\mathrm{SH})$ group functionalized long- alkyl chain on a clean gold $(\mathrm{Au})$ surface. The driving forces are: i) the formation of S-Au bonds (40-50 kcal/mole) and ii) lateral Van der Waals interactions among the aliphatic chains of neighboring thiol molecules. In order to build SAM's layer for PECs, donor-acceptors have to be covalently linked to thiol groups ${ }^{26,27}$. Fullerenes functionalized with thiol groups were used to form SAM's on gold surface by Echegoyan and his coworkers ${ }^{28}$. The photoaction spectrum of the fullerene self-assembled gold electrode was very similar to absorbance of the electrode in $0.1 \mathrm{M} \mathrm{KCl}$ solution. Later SAM's of fullerene using thiophene spacers with one-armed or 3-armed thiol group have been reported as shown in the Figure $1.10 .^{29,30}$ The anodic photocurrent density of 
PECs based on Au/trithiol was about 6 times larger than Au/monothiol. Improved performance was attributed to enhanced surface coverage.

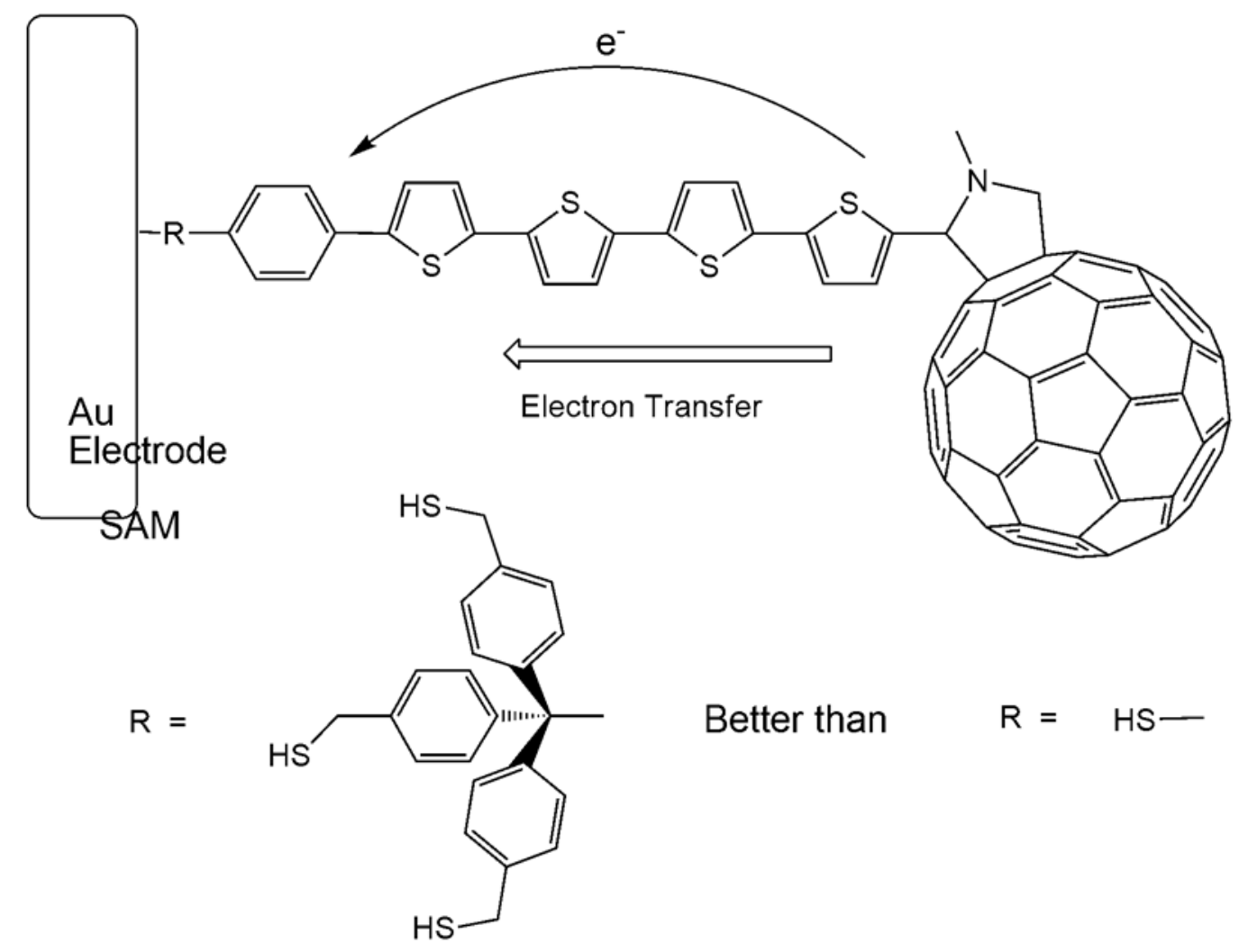

Figure 1.10: SAMs of fullerene appended monothiol and trithiol on gold electrode surface.

Imahori and coworkers reported cathodic photocurrent generation using SAMs of a covalently linked ferrocene-porphyrin-fullerene triads $\left(\mathrm{Fc}-\mathrm{P}-\mathrm{C}_{60}\right)$ on $\mathrm{Au}$ electrode ${ }^{31-34}$. PEC consisted of Au/SAMs as working electrode, methyl viologen as mediator and platinum counter electrode. Though the photosystem had highest photon quantum yield of more than $50 \%$, the IPCE of the system was still less than $1 \%$ with an applied potential of $200 \mathrm{mV}$ vs $\mathrm{Ag} / \mathrm{AgCl}$. They also showed enhanced performance due to better light capturing upon incorporating borondipyrrin (BDP) based thiol along with the triad as shown in Figure 1.11. In 2003, SAMs of porphyrin-fullerene dyad on indium tin oxide was reported to be more efficient than Au as a substrate. The same group also 
designed a system to reverse the direction of electron flow by switching the position of thiol group close to fullerene ${ }^{35}$. Thus SAMs have been very useful tool to manipulate the orientation of monolayered donor-acceptor films in a specific direction for PECs unlike LB films ${ }^{36}$.

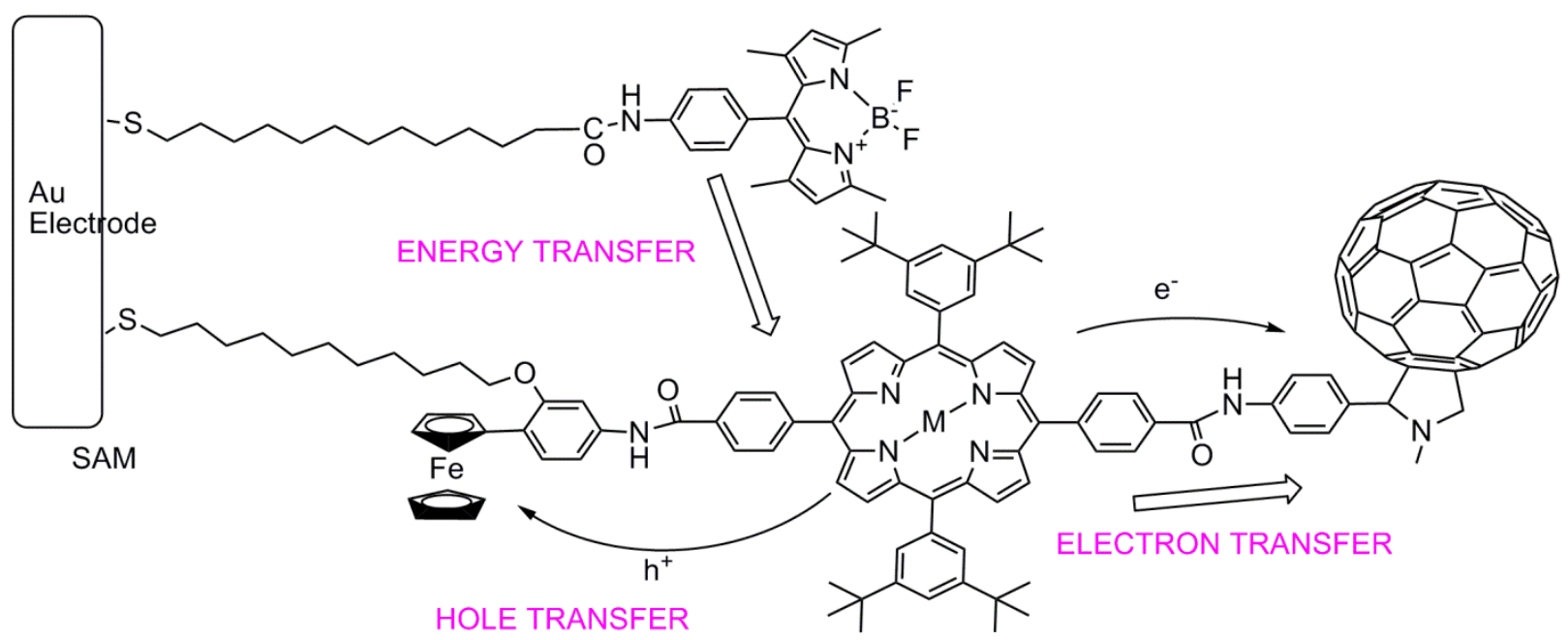

Figure 1.11: Co-self assembled monolayer of ferrocene-porphyrin-fullerene triad and boron dipyrin on gold surface.

\subsubsection{Electrophoretic Deposition}

Electrophoretic deposition is a simple technique to deposit charged macromolecule or colloids in solutions on the oppositely charged electrode surface in presence of a D.C potential. Kamat and his coworkers ${ }^{37,38}$ reported the electrophoretic deposition of fullerene nanoclusters on a tin oxide surface for the first time. The detailed steps are shown in the Figure 1.12. In short, fullerenes dissolved in toluene are injected into the acetonitrile solution. It results in the formation of a negatively-charged fullerene cluster, which then will be deposited on a surface by applying a dc electric field (100$200 \mathrm{~V})$. 


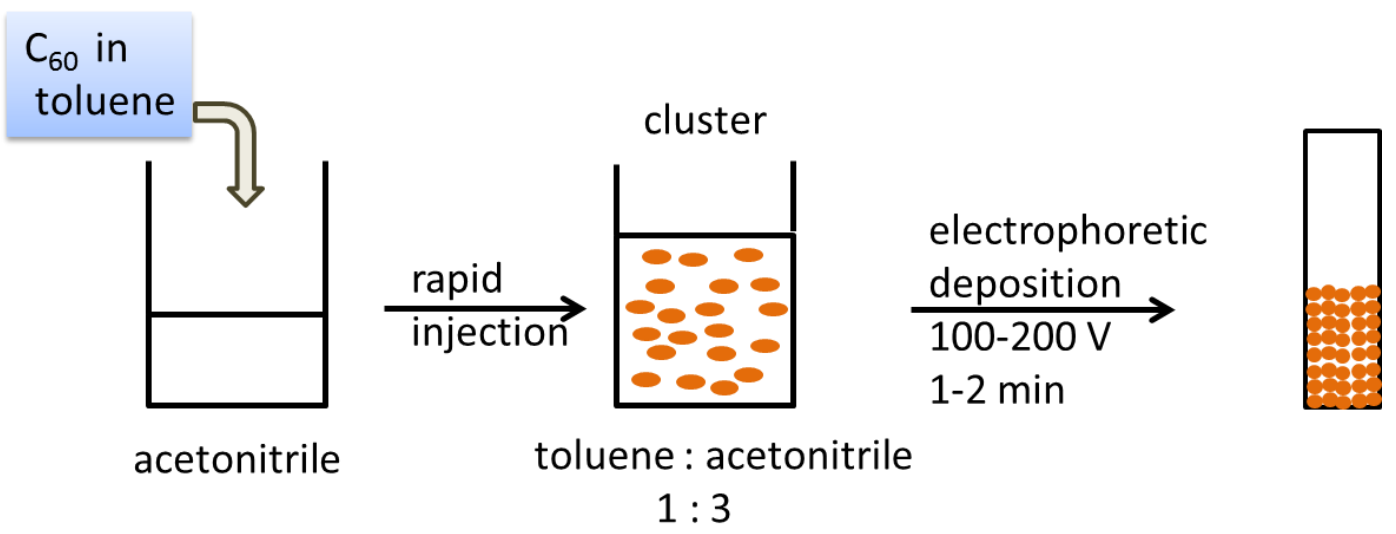

Figure 1.12: Schematic diagram electrophoretic deposition of fullerene on a conductive electrode surface.

Kamat and coworkers also reported the fullerene deposited electrode photoelectrochemistry with an IPCE of around $4 \% .{ }^{37,38}$ Similarly Imahori ${ }^{39-41}$ and his coworkers demonstrated electrophoretic deposition of porphyrin and phthalocyanine on $\mathrm{TiO}_{2}$. They reported an IPCE value of $2 \%$ using the iodide/iodine redox mediator for porphyrin/ $/ \mathrm{TOO}_{2}$ system. The phthalocyanine/ $/ \mathrm{TiO}_{2}$ electrode showed poor performance due to strong aggregation and resulted in self-quenching. Later different kinds of covalently bound donor - fullerene dyads were electrophoretically deposited on tin oxide surface to study the photoelectrochemical properties and compared with fullerene. Donor linked fullerene such as (N,N-dimethylaniline, N,N-dimethyl-p-toluidine, ferrocene, N-methylphenothiazine, and N,N-dimethyl-p-anisidine) and 1,2,5triphenylpyrrolidinofullerene and porphyrin-fullerene (Figure 1.13a) deposited electrode did not show significant improvement in performance compared pristine fullerene ${ }^{42}$. This might be due to uncontrollable molecular packing of the dyads on the electrode surface upon cluster formation for better electron injection and hole separation. But PECs based on composite clusters such as electrophoretic deposition of formanilide-anthraquinone dyad with $\mathrm{C}_{60}{ }^{43}$, adsorption of 9-mesityl-10-carboxymethylacridinum ion on $\mathrm{SnO}_{2}$ 
followed by $\mathrm{C}_{60}$ electrophoretic deposition showed improved performance ${ }^{44}$ with applied bias voltage. Electrophoretic deposition opened up the possibilities for increasing the surface coverage of donor-acceptor on metal oxide surface as in Figure 1.13b. In this technique ${ }^{45}$, covalently linked dyads must form charged cluster in a mixed solvent system in order to be deposited and employed for the application of PECs.

(a)

DONOR ACCEPTOR DYAD
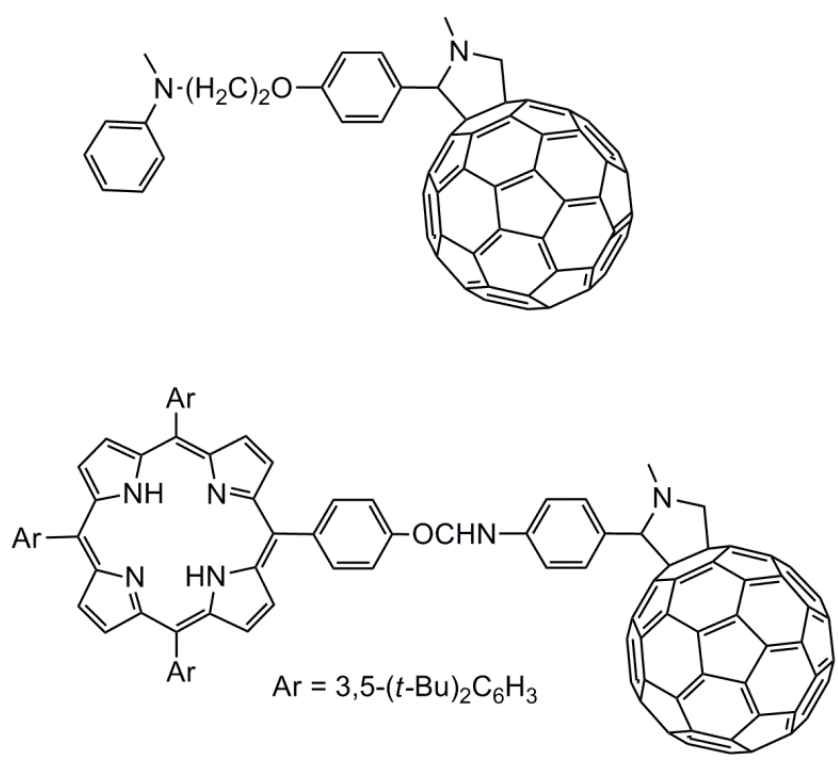

(b)

\section{COMPOSITE CLUSTERS}<smiles>O=C(c1ccccc1)c1ccc2c(c1)C(=O)c1ccccc1C2=O</smiles>

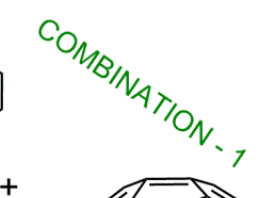

$+$
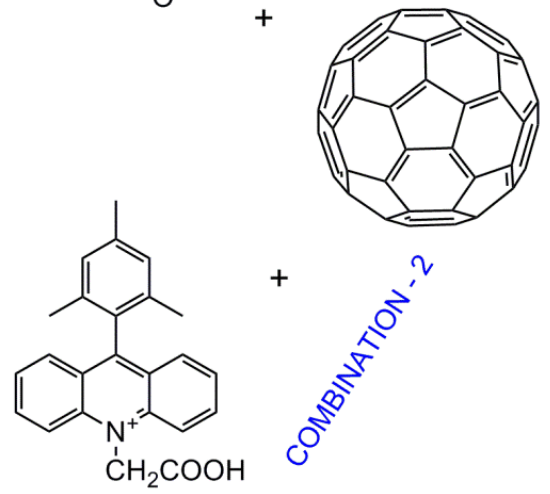

Figure 1.13: a) Model example of donor-acceptor dyad used for electrophoretic deposition. b) Composite cluster used in different combination along with electrophoretic deposition of fullerene.

\subsubsection{PECs Based on Non-Covalent Systems}

Various interaction such as ion-pairing, pi-pi stacking, hydrogen bonding and axial coordination have been used to self-assemble the donor and acceptor. ${ }^{12,46-48}$ Such interactions based PECs are briefly discussed below with appropriate film formation methods. 


\subsubsection{1 lon-Pairing}

Electrostatic attraction between two oppositely charged ions was cleverly utilized to form donor acceptor thin films using a layer-by-layer technique ${ }^{49-51}$. A classic example was the work of Guldi ${ }^{49,51}$ and his coworkers wherein they modified indium tin oxide (ITO) conducting surface with PDDA as shown in Figure 1.14. Van der Waals interactions between the $\mathrm{C}_{60}$ and PDDA facilitated the formation of first layer with net negative charge. The second step involved ion-pairing with positively charged octacationic porphyrin. This was followed by a third layer negatively charged metalated porphyrin. The fourth layer was formed using positively charged ferrocene. When light was shined, energy transfer between the free base and metalated porphyrin followed by sequential electron transfer between ferrocene-porphyrin and $\mathrm{C}_{60}$-porphyrin occurred. IPCE of $1.6 \%$ was reported for this layer without any bias voltage. Using the same concept ion pairing PECs based fullerene anion with positively charged cytochrome-c has been reported. ${ }^{52}$ Thus ion pairing serves as a tool for the formation of multilayered donor- acceptor system. ${ }^{50,53}$ The orientation was controlled by the using positive and negative charges incorporated in the molecules.

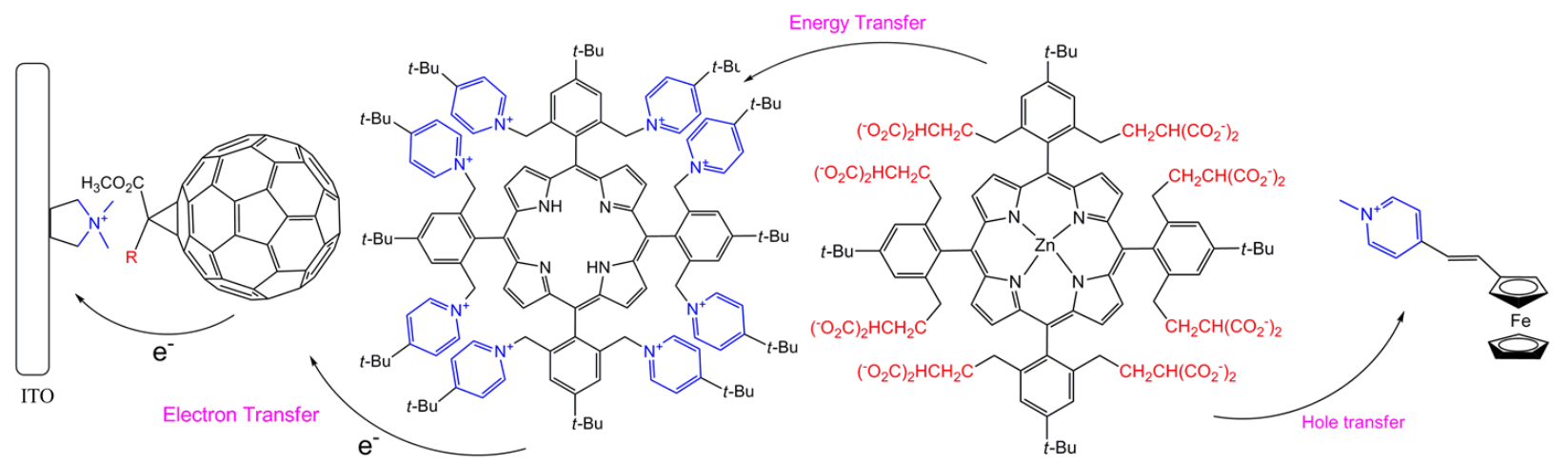

Figure 1.14: Schematic diagram of photoelectrochemical cell self assembled using ionion interaction between donor and acceptor. 


\subsubsection{Pi-Pi Stacking}

Pi-pi stacking is a weak electrostatic interaction occurring between the electron rich and electron poor aromatic rings. This attractive interaction is proportional to the contact surface area of the two pi-systems. Ground state and excited state interactions between porphyrin and fullerene upon pi-pi stacking have been reported. ${ }^{54,55}$ Hasobe $^{41}$ and his coworkers, electrophoretically deposited the pi stacked donor-acceptor on a tin oxide surface as shown in schematic Figure 1.15. Such an electrode showed an IPCE of close to $17 \%$ with an applied potential of $0.2 \mathrm{~V}$ in $\mathrm{Nal} / \mathrm{I}_{2}$ electrolyte system.

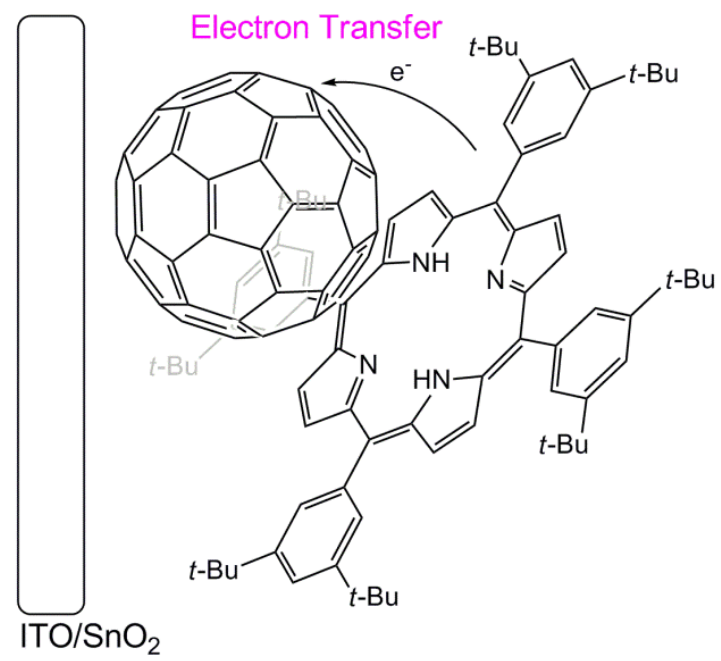

Figure 1.15: Schematic diagram of photoelectrochemical cell based on pi-pi interaction between donor and acceptor.

\subsubsection{Hydrogen Bonding}

It is a type of dipole-dipole interactions in which a hydrogen atom attached to an electronegative atom is attracted to a neighboring dipole on an adjacent molecule or functional group. Because it is highly directional and relatively strong, it is called 'master key interaction in supramolecular chemistry ${ }^{1}$. Tkachenko and his coworkers designed porphyrin-fullerene based hydrogen bonding system ${ }^{56}$. They spin coated the corresponding solution on an ITO electrode and formed the film. IR spectra for modified 
electrode revealed the presence of hydrogen bonding between the donor and the acceptor. Such a PECs in an air saturated $0.1 \mathrm{M} \mathrm{Na}_{2} \mathrm{SO}_{4}$ containing methyl viologen showed a quantum yield of $4 \%$. Basani ${ }^{57}$ and coworkers reported improved performance of 2.5 fold of the photovoltaic device based on hydrogen bonding between the melamine terminated donor oligomers and a complimentary barbiturate labeled electron acceptor fullerene. Imahori ${ }^{58}$ also have reported hydrogen bonded systems based on porphyrin containing carboxylic acid and appropriately functionalized fullerene spin coated on $\mathrm{TiO}_{2}$ surface. Compared to non-hydrogen bonded electrode, this photoelectrode showed higher IPCE and energy efficiency of $2.5 \%$. $\mathrm{Li}^{59}$ and his coworkers also reported hydrogen bonded supramolecular device based on perylene bisimide and fullerene derivatives shown below in Figure 1.16.

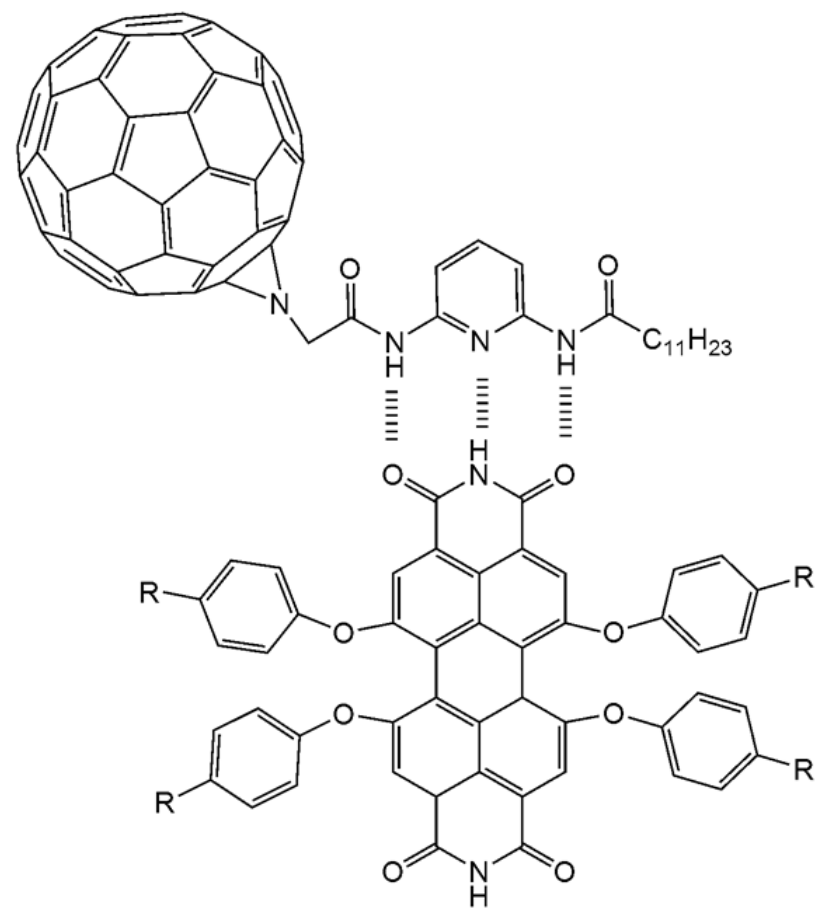

Figure 1.16: Schematic diagram of hydrogen bonding between the fullerene and perlyene diimide. 


\subsubsection{Coordination Bonding Systems}

Axial coordination involves a coordinating ligand and metal center usually in a tetrapyrrole macrocycle such as porphyrin, naphthalocyanine and phthalocyanine ${ }^{60}$. D'Souza $^{61}$ and his coworker reported LB film of axial coordinating imidazole functionalized fullerene with cationic zinc porphyrin as shown in Figure 1.17a. By switching the order of film formation, they were able to show both cathodic and anodic photocurrent using the same donor and acceptor. This shows advantage of supramolecular system: easy to modify the systems. Recently, Imahori ${ }^{62}$ reported axial coordinating vertically arranged donor-acceptor arrays based on porphyrin and functionalized fullerene on the tin oxide surface with an IPCE of $21 \%$. Kamat ${ }^{63}$ and his coworkers reported polypeptide chain with zinc porphyrin axially coordinating with $\mathrm{C}_{60}$ containing imidazole. The solution based studies revealed better charge separation, which was confirmed by the optical and transient techniques in solution. The dyad containing oligomers were electrophoretically deposited on $\mathrm{SnO}_{2}$. Such a system showed maximum IPCE of $56 \%$ at the porphryin Soret band ${ }^{63}$. Axial coordination between the oxygen atom and aluminium porphyrin has been reported by Hasobe and his coworker ${ }^{64}$. Electrophoretic deposition of aluminum porphyrin-fullerene dyad (Figure 1.17b) showed better IPCE performance from $400-600 \mathrm{~nm}$ compared to other zinc porphyrin-fullerene dyads. Also, supramolecular complexes of multiple porphyrin containing dendrimers, porphyrin appended gold nanoparticles combined with fullerene have been electrophoretically deposited. ${ }^{65-70}$ The dyad electrodes out performed in all the situations when compared to porphyrin alone or fullerene alone systems. 
(a)



(b)

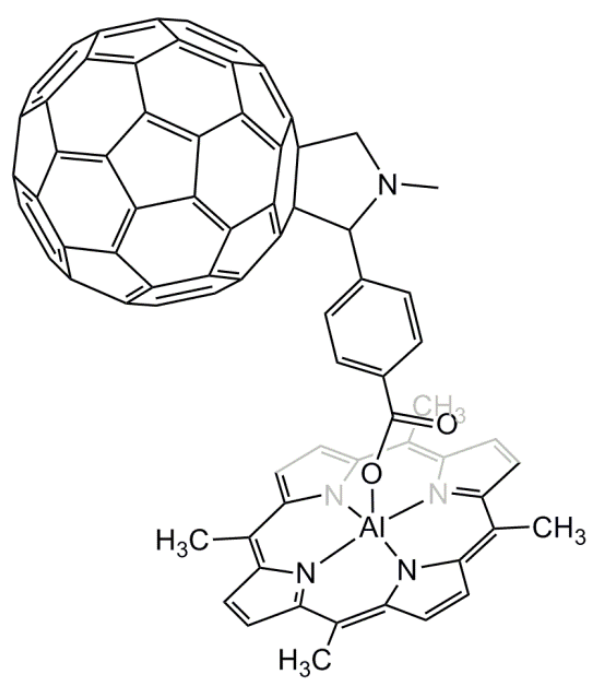

Figure 1.17: a) Axial coordination of fullerene derivative and water soluble zinc porphyrin. b) Axial coordination between fullerene and aluminum porphryin.

\subsection{Scope of the Present Work}

As mentioned in the preceding sections, with the techniques that were used for the construction of covalently linked PECs monolayer thick films could be formed effectively. ${ }^{19,32,34,71-73}$ In the case of multilayered films, lack of connection between the layers resulted in poor performance. To overcome, unconventional ways of forming donor-acceptor films are necessary.

Dye sensitized solar cells are the updated version of photosensitized PECs where the donor is the dye molecule and titanium dioxide nanoparticles could be considered as the acceptor instead of fullerene as discussed above. Detailed authoritative monographs, reviews and advance volumes on various aspects of DSSCs are available in literature. ${ }^{4,74-77}$ It all started after the most noticeable work published by Gratzel and his co-workers in $1993^{76}$. They reported nearly $10 \%$ energy efficient DSSC using an inorganic ruthenium dye (N3) adsorbed on mesoporous titanium dioxide film. Until now, no other inorganic complex could potentially replace the Ru dyes for DSSCs 
application. Although modified version of N3 showed better performance such as N719 and so called black dye. So to replace the expensive Ru based dyes, organic dye molecule of varying sizes have also been explored ${ }^{74}$. Of the plethora of organic molecule, dyes designed with the concept of donor-bridge-acceptor showed superior performance. An example of high performance dye ( $9.8 \%$ energy efficiency) is shown in the Figure 1.18 a where donor is triphenyl ${ }^{78}$ amino group and acceptor is cyano acrylic acid group. The acid group is used for adsorbing the dye on the electrode surface. Another important factor to be considered is aggregation of dye molecules specially when designing tetrapyrrole based dye. A well-designed zinc porphyrin ${ }^{79}$ based dye is shown in the Figure.1.18b whose reported efficiency $(11 \%)$ is superior to all other other organic dyes. Careful considerations on the design of an efficient system should include the following factors:

- Good electronic communication (triple bond) between the donor (zinc porphyrin) and acceptor anchoring group (carboxylic acid).

- Tert-butyl groups on the meso positioned phenyl groups to prevent the aggregation upon adsorption.

- N,N diaryl amino group on the opposite side of anchoring group to enable push pull electron mechanism and also extends the conjugation. 
(a)

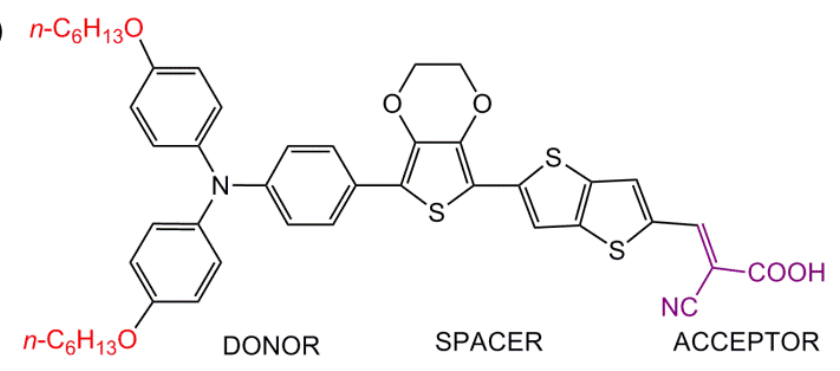

(b)

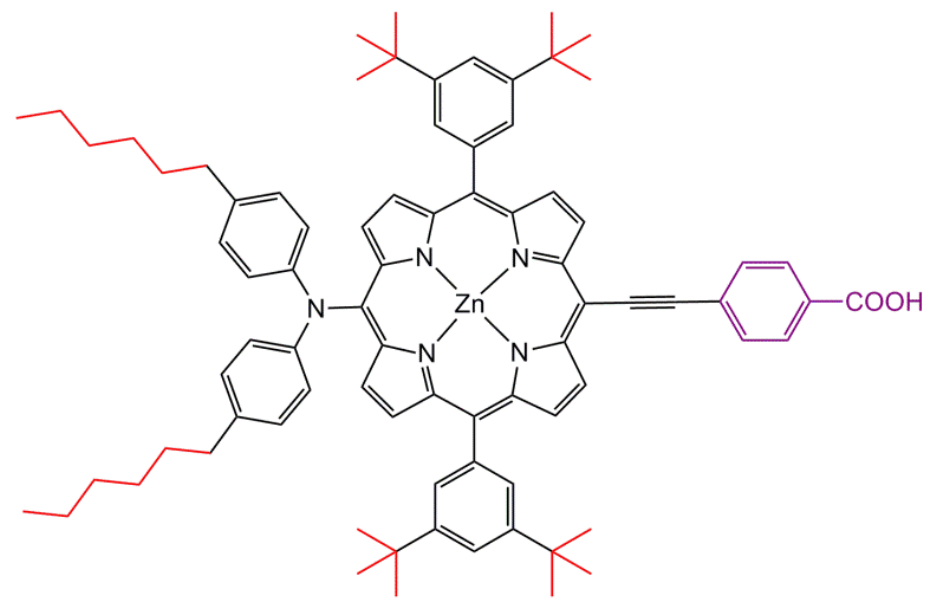

Figure 1.18 a) Donor-acceptor based organic dye for dye sensitization. b) Porphyrin based carboxylic acid dye used for dye sensitized solar cells.

Despite these high performances, continuous efforts are currently being made to build dye sensitized solar cells capable of using entire solar energy from visible to near IR region. This is possible with effective design of a dye that is being adsorbed on the metal oxide ${ }^{80-86}$. One magic broad band capturing dye with suitable above mentioned properties is yet to be synthesized. Multiple interactions between the dyes similar to that in photosynthetic reaction center pigment molecules could be used for designing broad band-capturing systems. Since supramolecular chemistry is the chemistry of interactions, it is more important to design, construct and understand solar cells based on supramolecular interactions.

Most of the dyes used for adsorption on $\mathrm{TiO}_{2}$ were having carboxylic acid as an anchoring group ${ }^{74}$. The carboxylic acid based dyes should be designed with the 
consideration of multiple interactions. Also, it is required to explore the other possibilities for dye sensitization in DSSCs to extend the energy capturing abilityy. With the help of supramolecular chemistry and with new biomimetic methods of sensitization unusual compounds, for example dimeric pigment molecules in photosynthetic systems, should be made available for solar applications

DSSCs' improved performance originated from the usage of nanoparticles to increase the surface area. As a result, the amount of dye that is adsorbed and the performance was enhanced. Similarly, supramolecular dye loading could be improved using metal oxide. This is an important factor to be considered for designing PECs based supramolecular system which was lacking in previously designed systems. In addition, suitable orientation and position of the supramolecules on the metal oxide surface is important to ensure unidirectional electron movement.

An attempt to address all of the points mentioned above forms the central theme of this thesis and systematically and discussed in 8 chapters.

In chapter 1, a brief introduction about the supramolecular chemistry and the bio supramolecular applications for energy harvesting was discussed. Then the concept of photoinduced electron transfer phenomenon and its significance are reviewed. The types of photoelectrochemical cells and the key parameter to characterize the solar cell are shown. A brief and effective literature review of photoelectrochemical systems based on covalent and non-covalent interactions is performed. Finally, it ends with discussing the scope of the present work.

Chapter 2 provides the information about the commonly used chemicals, solvents, materials and instrumentation methods. Also the preparation procedure of thin 
films including $\mathrm{TiO}_{2}, \mathrm{SnO}_{2}$ and their characterization are given. Detailed descriptions of the used calculations are also reported.

In chapter 3 , the photoelectrochemical behavior of $\mathrm{C}_{60}$ self-assembled via axial coordination to an electrochemically polymerized zinc porphyrin film is systematically investigated to unravel the importance of the coordinated fullerene in improving the photocurrent and photovoltage generation of the resulting donor-acceptor dyad. For this, tetrakis(4-(N,N-diphenylamino)-phenyl)porphyrinatozinc(II), $\left(\mathrm{Ph}_{3} \mathrm{~N}\right) \mathrm{ZnP}$, bearing electroactive triphenylamine peripheral substituents is first electropolymerized to form a film on the electrode surface. The resulting formation of the electrochemically active and dense film is confirmed by using an electrochemical quartz crystal microbalance (EQCM) and by AFM imaging. The optical absorption and emission studies revealed the characteristic absorption and emission bands of zinc porphyrin that suggest preserving the $\pi$-electron system of the porphyrin monomer in the polymer. Further, fullerene, derivatized with an axially coordinating imidazole ligand, is allowed to selfassemble via axial coordination to the zinc center of the $\left(\mathrm{Ph}_{3} \mathrm{~N}\right) \mathrm{ZnP}$ polymer film. The simultaneously performed piezoelectric microgravimetry and cyclic voltammetry studies using EQCM allowed us to prove this coordination and to evaluate the redox potential of both the donor, $\left(\mathrm{Ph}_{3} \mathrm{~N}\right) \mathrm{ZnP}$, and acceptor, $\mathrm{C}_{60}$, moiety. Systematic photoelectrochemical studies reveal cathodic photocurrent generation, a result unlike most of the dyesensitized photoelectrochemical cells reported in the literature. Moreover, the coordinated fullerene to the zinc porphyrin film improved both the photocurrent and photovoltage generation of the photoelectrochemical cell. An incident photon-to-current 
conversion efficiency (IPCE) of nearly $2 \%$ at the Soret region of maximum absorption is determined for the $\left[\left(\mathrm{Ph}_{3} \mathrm{~N}\right) \mathrm{ZnP}\right.$ polymer]-fullerene hybrid film.

Chapter 4 is divided into 2 parts. In Part 1 , to improve the performance of axially coordinating system, an elegant method of self-assembly for modification of a $\mathrm{TiO}_{2}$ surface using coordinating ligands followed by immobilization of variety of sensitizers and a dyad is reported. This highly versatile method, in addition to testing the photoelectrochemical behavior of different zinc tetrapyrroles has allowed the use of fairly complex structures involving more than one donor entity. Utilization of the zinc porphyrin-ferrocene dyad markedly improved the current-voltage performance of the photoelectrochemical cell through an electron transfer-hole migration mechanism. Incident photon-to-current efficiency values up to $37 \%$ are obtained for the electrode modified with the dyad, signifying the importance of photocells built on the basis of biomimetic principles for efficient harvesting of solar energy.

In part 2, effect of replacement of $\mathrm{Zn}$ metal atom with magnesium in porphyrin macrocycle is reported. The performance of magnesium porphyrin is almost equal to that of the zinc porphyrin-ferrocene dyad. Optimization of solvent conditions for axial coordination is also reported with improved fill factor from $49 \%$ to $71 \%$. Improvement was due to decrease in resistance of the electrolyte under mixed solvent system condition. Later is confirmed using electrochemical impedance spectroscopy.

Chapter 5 is based on multi-interaction supramolecular systems using oxoporphyrinogen, porphyrin and fullerene and it has 3 parts. The 2 interactions are axial coordination and anion binding. Detailed solution studies and photoelectrochemical experiments are discussed. 
Part 1 showed the effect of strongly binding fluoride $\left(\mathrm{F}^{-}\right)$anion stabilizing the photo-induced charge-separated states of a bis-fullerene-substituted oxoporphyrinogen due to the large shift in the oxidation potential of the oxoporphyrinogen moiety upon anion binding through hydrogen bonding at its core.

Part 2 involves a study of an interesting supramolecular oligochromophoric system possessing exclusive binding sites for both a guest electron acceptor and an anionic cofactor species. Anion-binding-induced stabilization of charge separated state is successfully demonstrated in this part. Towards this, intramolecular and intermolecular photochemical processes of a supramolecular complex of a bisporphyrinyl-substituted oxoporphyrinogen with a bis(4-pyridyl)-substituted fullerene are investigated by using spectral, electrochemical, femtosecond and nanosecond laser flash photolysis and transient absorption measurements. In the case of fluoride anion binding, anion-complexation-induced stabilization of charge separation resulted a 90 fold elongation of the CS state lifetime from $163 \mathrm{~ns}$ to $14 \mu \mathrm{s}$. Complexation of anions to the oxoporphyrinogen center lowers its oxidation potential by nearly $600 \mathrm{mV}$ creating an intermediate energy state for charge migration from the $\mathrm{ZnP} \cdot+$ to the oxoporphyrinogen anion complex.

Part 3 discusses a novel approach for improving photocurrent in a supramolecular solar cell, composed of zinc porphyrin-oxoporphyrinogen (ZnP-OXP) surface-modified $\mathrm{TiO} 2$, by redox tuning through fluoride anion binding to the redox active host, OXP. Anion bound (ZnP-OXP) system showed improvement in performance due to the shift in redox potential as in part 1 and 2 . This study particularly demonstrates the photoelectrochemical application of anion binding. 
In chapter 6, ion-ion pairing concept is used as method of sensitization of tin oxide nanoparticles. Thin transparent $\mathrm{SnO}_{2}$ films have been surface modified with cationic water soluble porphyrins for photoelectrochemical investigations. Free-base and zinc(II) derivatives of three types of cationic water soluble porphyrin are used. The negative surface charge and the porous structure of $\mathrm{SnO}_{2}$ facilitated binding of positively charged porphyrins such as tetrakis( $\mathrm{N}$-methylpyridyl)porphyrin chloride, (TMPyP)M via electrostatic interactions. The $\mathrm{SnO}_{2}$-porphyrin binding in solution is probed by absorption spectroscopy, steady-state and time-resolved fluorescence studies which revealed electron injection from singlet excited porphyrin to $\mathrm{SnO}_{2}$ conduction band. Photoelectrochemical studies performed on $\mathrm{FTO} / \mathrm{SnO}_{2} /(\mathrm{P}) \mathrm{M}$ electrodes reveal incident photon-to-current conversion efficiencies (IPCE) up to $91 \%$ at the peak maxima for the $\mathrm{SnO}_{2}$-dye modified electrodes, with very good on-off switching ability. The high IPCE values have been attributed to the strong electrostatic and electronic interactions between the dye, (TMPyP)M and $\mathrm{SnO}_{2}$ nanoparticles. Electrochemical impedance spectral measurements of electron recombination resistance calculations are supportive of this assignment.

Chapter 7 is the extension of chapter 6 wherein heterodimers are used for sensitization of tin oxide. Water soluble oppositely charged tetrapyrrole macrocycles such as zinc tetra $\mathrm{N}$-methyl pyridyl porphyrin and zinc phthalocyanine sulfonic acid are manipulated to form stable ion-paired hetero-dimers. Such dimers are formed due to coulombic and hydrophobic interactions. They have different characteristic absorbance than individual tetrapyrrole macrocycles as shown by absorption studies. Steady state emission studies revealed excellent electron transfer upon formation of dimer. Surface 
modification of tin (IV) oxide with negatively charged phthalocyanine sulfonic acid followed by ion pairing of positively charged porphyrin resulted in successful selfassembly of dimers on tin-oxide surface. Upon illumination Zinc Phthalocyanine Sulfonic acid (ZnPcS) - Zinc Tetra Methyl N-Pyridyl Porphyrin (ZnTMPyP) dimer resulted in higher shortcut circuit current compared to corresponding individuals. Higher Incident Photon Conversion Efficiency more than $50 \%$ from $400-700 \mathrm{~nm}$ and $80 \%$ at the 450 $\mathrm{nm}$ is reported for the supramolecular self- assembled ZnTMPyP-ZnPcS heterodimer. Improved performance upon dimer formation was attributed to the increase in recombination resistance and better light harvesting property as confirmed by electrochemical impedance spectroscopy.

Finally chapter 8 summarizes the obtained results and their significance. 


\section{CHAPTER 2}

\section{MATERIALS AND PHYSICAL METHODS}

This chapter presents list of chemicals, solvents, and materials employed at the various stages of research work: general procedure used for the purification of solvents, materials and chemicals, general synthetic procedure employed for the synthesis of porphyrin, fullerene derivatives, brief discussion of analytical methods employed and also calculations used during the course of studies are presented here.

\subsection{Materials}

Fullerene, $\mathrm{C}_{60}(+99.95 \%)$, was from SES Research, (Houston, TX). All the reagents were from Aldrich Chemicals (Milwaukee, WI) while the bulk solvents utilized in the syntheses were from Fisher Chemicals. Other chemicals including chloroplatinic acid, iodine, tert-butyl pyridine, tetra-n-butylammonium acetate, tetra-n-butylammonium fluoride, tetra-n-butylammonium bromide, tetra-n-butylammonium phosphate, tetra-nbutylammonium nitrate, benzoic acid, tetra- n-butylammonium nitrate, iodine, titanium tetrachloride and isonicotinic acid were from Sigma Aldrich. Colloidal $\mathrm{SnO}_{2}$ and fluoropyridine carboxylic acid was purchased from Alfa Aesar. Water soluble porphyrins and phthalocyanines were bought from Frontier scientific chemicals. Tetra-nbutylammonium perchlorate, (TBA) $\mathrm{ClO}_{4}$, and tetra-n-butylammonium iodide, (TBA)I, used as supporting electrolytes in electrochemical experiments were bought from Southwest Chemicals. Optically transparent conducting glass (FTO Tec7) electrodes were purchased from of Pilkington North America Inc. (Toledo, $\mathrm{OH}$ ). $\mathrm{TiO}_{2}$ pastes and N3 dye used to build DSSCs were purchased from Dyesol. 


\subsection{Optical Physical Methods}

\subsubsection{Spectroscopic Studies}

The UV-visible spectral measurements were carried out with the Model 1600 UVVis or UV2501PC UV-vis recording spectrophotometer of Shimadzu (Kyoto, Japan) or V670 UV-visible-near IR spectrophotometer of Jasco (Great Dunmow, Essex, UK). The fluorescence emission was monitored by using a Varian Eclipse spectrometer. Rightangle fluorescence was detected while placing the substrate at angle of $45^{\circ}$ to the excitation beam. Lifetime and solid state fluorescence emission were recorded using Horiba Jobin Yvon Nanolog UV-visible NIR spectrofluorometer with time correlated single photon counting (TCSPC) lifetime option with nano-LED excitation sources (excitation pulse width 100-200 ps). ${ }^{1} \mathrm{H}$ NMR spectra were obtained from chloroform$\mathrm{d}_{1}$ solutions using a Varian $400 \mathrm{MHz} \mathrm{NMR}$ spectrometer with tetramethylsilane as an internal standard.

\subsubsection{Transient Techniques}

Time-resolved transient absorption measurements were measured in Prof. Fukusuzumi's Lab at Osaka University, Japan. Femtosecond laser flash photolysis was conducted using a Clark-MXR 2010 laser system and an optical detection system provided by Ultrafast Systems (Helios). The source for the pump and probe pulses were derived from the fundamental output of Clark laser system $(775 \mathrm{~nm}, 1 \mathrm{~mJ} /$ pulse and $\mathrm{fwhm}=150 \mathrm{fs}$ ) at a repetition rate of $1 \mathrm{kHz}$. A second harmonic generator introduced in the path of the laser beam provided $410 \mathrm{~nm}$ laser pulses for excitation. A $95 \%$ of the fundamental output of the laser was used to generate the second harmonic, while $5 \%$ of the deflected output was used for white light generation. Prior to generating 
the probe continuum, the laser pulse was fed to a delay line that provided an experimental time window of $1.6 \mathrm{~ns}$ with a maximum step resolution of $7 \mathrm{fs}$. The pump beam was attenuated at $5 \mu \mathrm{J} /$ pulse with a spot size of $2 \mathrm{~mm}$ diameter at the sample cell where it was merged with the white probe pulse in a close angle $\left(<10^{\circ}\right)$. The probe beam after passing through the $2 \mathrm{~mm}$ sample cell was focused on a $200 \mu \mathrm{m}$ fiber optic cable which was connected to a CCD spectrograph (Ocean Optics, S2000-UV-vis for visible region and Horiba, CP-140 for NIR region) for recording the time-resolved spectra (450-800 and 800-1400 nm). Typically, 5000 excitation pulses were averaged to obtain the transient spectrum at a set delay time. The kinetic traces at appropriate wavelengths were assembled from the time-resolved spectral data.

Nanosecond time-resolved transient absorption measurements were carried out using the laser system provided by UNISOKU Co., Ltd. Measurements of nanosecond transient absorption spectrum were performed according to the following procedure. A deaerated solution containing a dyad was excited by a Panther OPO pumped by Nd:YAG laser (Continuum, SLII-10, 4-6 ns fwhm) at $\lambda=430 \mathrm{~nm}$. The photodynamics was monitored by continuous exposure to a xenon lamp (150 W) as a probe light and a photomultiplier tube (Hamamatsu 2949) as a detector. Transient spectra were recorded using fresh solutions in each laser excitation. The solution was deoxygenated by argon purging for 15 min prior to measurements.

\subsubsection{Cyclic Voltammetry}

CV experiments were performed using a PARSTAT 2273 Potentiostat/Galvanostat of AMETEK Princeton Applied Research (Oak Ridge, TN) controlled by the Powersuite software. A platinum disk of $0.5 \mathrm{~mm}$ diameter or ITO 
coated glass was used as the working electrode. A platinum wire or foil and an $\mathrm{Ag} / \mathrm{AgCl}$ electrode served as the counter and reference electrode, respectively. A ferrocene/ferrocenium $\left(\mathrm{Fe} / \mathrm{Fe}^{+}\right)$redox couple was used as the internal standard for potentials. The same potentiostat combined with Jasco spectrophotometer was used for recording spectroelectrochemistry.

\subsubsection{Electrochemical Quartz Crystal Nanobalance}

Simultaneous $\mathrm{C}$ and PM experiments were performed by using an EP-21 potentiostat of Elpan (Lubawa, Poland) connected to the EQCM 5710 electrochemical quartz crystal microbalance of the Institute of Physical Chemistry (Warsaw, Poland) under the EQCM 5710-S2 software control. This microbalance allowed for simultaneous batch measurements of changes of current, resonant frequency, and dynamic resistance as the function of potential or time of a $10-\mathrm{MHz}$, At-cut plano-plano quartz crystal resonator during potential scanning. The resonators featured $5-\mathrm{mm}$ diameter Au-over-Ti film circular electrodes. Piezoelectric microgravimetry experiments under FIA conditions were performed with the flow-through quartz crystal holder Model EQCM 5610 of the Institute of Physical Chemistry (Warsaw, Poland) hooked up to the EQCM 5710 controller, and connected with a stillness still tubing to a NE-500 syringe pump of New Era Pump Systems, Inc. (Wantagh, NY) under the RS-232 terminal emulator software control via a six-port injection valve Model 7725i of Rheodyne, (Cotati, CA). 


\subsubsection{Electrochemical Impedance Spectroscopy}

Electrochemical impedance measurements were performed using EG\&G PARSTAT 2273 potentiostat. Impedance data were recorded under forward bias condition from $100 \mathrm{kHz}$ to $100 \mathrm{mHz}$ with an A.C amplitude of $10 \mathrm{mV}$. Data were recorded under dark and A.M 1.5 illumination conditions applying corresponding $V_{\text {oc }}$ for each electrode. The data were analyzed using ZSimpwin software from Princeton Applied Research.

\subsection{Surface Characterization Techniques}

\subsubsection{Atomic Force Microscopy}

Tapping mode AFM imaging was performed with the Multimode NS3D microscope of Veeco Instruments, Inc. (Woodbury, NY) and thickness of the polymer films was determined using software of this manufacturer. AFM was recorded in Prof. Kutner's lab in Poland.

\subsection{Preparations of Metal Oxides}

\subsubsection{Cleaning of FTO}

FTO glass plates were cleaned using the detergent by gently rubbing over the micro cloth pads $(B A S)^{87}$. After washing the soap with DI water, they were further

cleaned using $0.1 \mathrm{M} \mathrm{HCl}$ in ethanol, acetone and 2-proapanol solutions in an ultrasonic bath for 15 min sequentially. The electrodes were then treated with $\mathrm{O}_{2}$ plasma cleaning for $10 \mathrm{~min}$ at medium power intensity to remove all the organic impurities and activate the surface. Plasma cleaning was performed using PDC - 32G from Harrick and Plasma using oxygen. 


\subsubsection{Preparation of Nanocrystalline $\mathrm{SnO}_{2}$ Electrodes}

These were prepared according to the literature procedure by Kamat and coworkers $^{88}$ with few changes. A $10 \mu \mathrm{L}$ of $\mathrm{SnO}_{2}$ colloidal solution (Alfa Aesar, 15\%) was dissolved in $10 \mathrm{~mL}$ of ethanol; a $500 \mu \mathrm{L}$ of $\mathrm{NH}_{4} \mathrm{OH}$ was added to this solution for stability. About $2 \mathrm{~mL}$ of colloidal solution placed on optically transparent electrode, fluorine doped indium tin oxide (FTO) (Pilkington TEC-8, 6-9 $\Omega /$ square) and dried in air on a warm plate. The electrodes were annealed in an oven for an hour in air at $673 \mathrm{~K}$.

\subsubsection{Preparation of $\mathrm{TiO}_{2}$}

Thin films of $\mathrm{TiO}_{2}$ on FTO was developed using according literature procedure. ${ }^{87}$ The electrodes were then heated at $450^{\circ} \mathrm{C}$ to remove all the organic impurities. Then, a layer of $\mathrm{TiO}_{2}(18 \mathrm{NRT})$ or (18NR AO) paste was coated on the FTO glass plates using Doctor Blade techniques. After leaving electrodes for $10 \mathrm{~min}$ to reduce the surface irregularity, they were heated gradually from $120^{\circ} \mathrm{C}$ to $550^{\circ} \mathrm{C}$. After annealing, the $\mathrm{TiO}_{2}$ films were treated with $40 \mathrm{mM} \mathrm{TiCl}_{4}$ solution at $70^{\circ} \mathrm{C}$ for $30 \mathrm{~min}$, rinsed with water and ethanol, followed by annealing at $520^{\circ} \mathrm{C}$. Then $\mathrm{TiO}_{2}$ electrodes were cooled to $80^{\circ} \mathrm{C}$ and used it for dye sensitization.

\subsubsection{Preparation of Counter Electrode}

The cleaned FTO was heated at $440{ }^{\circ} \mathrm{C}$ for 15 minutes ${ }^{87}$. Then the counter electrode was formed by depositing the Pt catalyst on the FTO glass (Tec 8, Pilkington) using a drop $\mathrm{H}_{2} \mathrm{PtCl}_{6}$ solution (5 $\mathrm{mg} \mathrm{Pt}$ in $1 \mathrm{ml}$ ethanol). Once the solvent was evaporated, it was heated at $400{ }^{\circ} \mathrm{C}$ for $15 \mathrm{~min}$. Cooled electrode was used for the preparation of DSSCs. 


\subsection{Photoelectrochemistry}

Two types of photoelectrochemical experiments were performed in a twoelectrode configuration. One type was similar to DSSC charatacterization, where the two electrodes were clamped together and a drop of electrolyte was added. The area of the electrode was controlled using a mask. Second one is a photoelectrochemical setup that involves $\mathrm{FTO} / \mathrm{SnO}_{2}$ electrode and a platinum foil as a counter electrode. The 2 electrodes are separated using $0.7 \mathrm{~cm}$ Teflon spacer and placed in a typical UV cuvette. The electrolyte was added in between the two electrodes and the area of electrode was controlled by using a mask on the photoelectrode side of cuvette.

The photocurrent-photovoltage $(I-V)$ characteristics ${ }^{89}$ of the solar cells were measured using a Model 2400 Current/Voltage Source Meter of Keithley Instruments, Inc. (Cleveland, $\mathrm{OH}$ ) under illumination with an $\mathrm{AM} 1.5$ simulated light source using a Model 9600 of 150-W Solar Simulator of Newport Corp. (Irvine, CA). A 340-nm filter was introduced in the light path to eliminate UV radiation. The light intensity was monitored by using an Optical Model 1916-C Power Meter of Newport. Incident photonto-current efficiency (IPCE) measurements were performed under $\sim 2.5 \mathrm{~mW} \mathrm{~cm}$ monochromatic light illumination conditions using a setup comprised of a $150 \mathrm{~W}$ Xe lamp with a Cornerstone 260 monochromator (Newport Corp., Irvine, CA).

\subsection{Calculations}

This section explains in detail the calculations that were used in these studies. Some of them were already discussed in the introduction chapter: Rehm - Weller equations, energy efficiency and quantum efficiency calculations. 


\subsubsection{Benesi-Hildebrand Plot}

Benesi-Hildebrand Plot was used to calculate the association constant or binding constant from the UV-Vis absorbance titrations. The equation used for calculation of binding constant is given below. ${ }^{90}$

$$
\frac{1}{\Delta A}=\frac{1}{\left[R^{0}\right] K \Delta \epsilon_{R S}\left[S^{0}\right]}+\frac{1}{\left[R^{0}\right] \Delta \epsilon_{R S}}
$$

$\left[R^{0}\right]$ and $\left[S^{0}\right]$ are the concentration of the receptor and substrate, $\Delta A$ is the difference in absorbance. The binding constant $K$ can be obtained from the values of slope and $y$ intercept in the plot between the difference absorbance $(\Delta \mathrm{A})$ and substrate concentration. $K$ is determined from (y-intercept) / (slope). Using the difference in absorbance and molar of ratio of substrate and receptor, the number of equivalents between the substrate and the receptor involved in the binding event was determined. This is called mole ratio plot.

\subsubsection{Computational Calculation}

To visualize the optimized structure and position of HOMO - LUMO of the supramolecular systems, geometry optimizations were performed by DFT B3LYP/3$21 \mathrm{G}\left({ }^{*}\right)$ methods with the GAUSSIAN 03 software package on a high-speed computer. The graphics of HOMO and LUMO coefficients were generated with the help of GaussView software.

\subsubsection{Light Harvesting Efficiency (LHE)}

LHE of the sensitized oxide layer is the efficiency of the electron injection from the sensitizer into the oxide layer. ${ }^{4}$ Here we used tin oxide as a model and it could be replaced with any oxide layer.

The LHE for the for photo-electro side illumination was given as 


$$
\text { L.H. } E_{P E}=\frac{(1-R) \alpha\left(1-e^{-\left(\alpha+\alpha_{I}\right) d}\right)}{\alpha+\alpha_{I}}
$$

where $R$ denotes the reflectance from the glass, $d$ is the thickness of the tin oxide layer and $\alpha$ is the absorption coefficient of the dye coated mesoporous film given by

$$
\alpha=-\left(\frac{1}{d}\right) \ln \left(\frac{T_{\mathrm{SnO}_{2}, \mathrm{Dye}}}{T_{\mathrm{SnO}_{2}}}\right)
$$

where $T_{\mathrm{SnO}_{2}, \mathrm{Dye}}$ and $T_{\mathrm{SnO}_{2}}$ are the transmittance of the electrode with and without the dye. Upon substituting (2) in (1)

$$
\text { L.H.E }=\frac{(1-R) \ln \left(\frac{T_{\mathrm{SnO}_{2}, \mathrm{Dye}}}{T_{\mathrm{SnO}_{2}}}\right) x\left(1-\frac{T_{\mathrm{SnO}_{2}, \mathrm{Dye}} * T_{\mathrm{SnO}_{2}, \text { Elec }}}{T_{\mathrm{SnO}_{2}}^{2}}\right)}{\ln \left(\frac{T_{\mathrm{SnO}_{2}, \mathrm{Dye}}}{T_{\mathrm{SnO}_{2}, \mathrm{Dye}}}\right)+\ln \left(\frac{T_{\mathrm{SnO}_{2}, \text { Elec }}}{T_{\mathrm{SnO}_{2}, \mathrm{Dye}}}\right)}
$$

where $T_{\mathrm{SnO}_{2}, \mathrm{Dye}}, \mathrm{TSnO}_{2}$ and $T_{\mathrm{SnO}_{2}, \text { Elec }}$ are the transmittance of the dye electrode, tin oxide alone and tin oxide, electrolyte. Assuming the transmittance of tin oxide with and without electrolyte to be same, the L.H.E. is calculated as shown below,

$$
\text { L.H.E }=(1-R)\left(1-\frac{T_{\mathrm{SnO}_{2}, \text { Dye }}}{T_{\mathrm{SnO}_{2}}}\right)
$$




\section{CHAPTER 3}

\section{SUPRAMOLECULAR DONOR-ACCEPTOR HYBRID OF ELECTROPOLYMERIZED ZINC PORPHYRIN WITH AXIALLY COORDINATED FULLERENE*}

\subsection{Introduction}

There has been a continuous interest in the synthesis and property studies of metalloporphyrins because of their widespread occurrence in nature, strong optical absorption and emission, and redox properties applicable for electron transfer reactions related to solar energy harvesting. ${ }^{11,12,60,91-99}$ Moreover, electronic properties of the porphyrin $\pi$-electron system can readily be altered by covalent attachment of electron donating or accepting axial or peripheral substituents, although these effects are more pronounced for substitution of the $\beta$-pyrrole macrocycles than the meso-phenyl rings. ${ }^{91,97-100}$ The diminished electronic effect in the latter derivatives is primarily due to the out-of-porphyrin-plane twisting of the meso-aryl groups that result in interruption of the $\pi$-conjugated macrocycle and alignment of the excited state dipoles.

Triphenylamine (TPA) $)^{101,102}$ is a strong chromophore, absorbing at around $300 \mathrm{~nm}$, due to the $\mathrm{n}$-to- $\pi^{*}$ electronic transition, and emitting at around $450 \mathrm{~nm}$. Both the monomer and polymer form of TPA, owing to the low oxidation potential and appreciable film-forming properties, have been widely used in the field of organic lightemitting diodes, information storage devices, hole-transporting materials, field-effect transistors, and photovoltaic cells ${ }^{103-106}$. In recent years, significant interest has been focused on the synthesis of hybrid molecules containing triarylamine as one of the

*Section 3 in its entirety is reproduced from the Journal of Physical Chemistry C, 2009, 113 (20), pp 89828989 with permission from the publisher. 
entities with other redox or photoactive molecular entities. ${ }^{107-109}$ In this context, only a few examples of porphyrin-triarylamine hybrids have been reported. ${ }^{110-114}$ Electronic properties of the porphyrin $\pi$-electron systems were significantly altered by the triarylamine meso-substituents. Recently, a TPA-porphyrin-fullerene multimodular donor-acceptor hybrid was constructed, ${ }^{115}$ in which sequential energy transfer, followed by electron transfer, was reported. In this hybrid, energy transfer from the singlet excited TPA to zinc porphyrin, $\mathrm{ZnP}$, followed by electron transfer from ${ }^{1} \mathrm{ZnP}{ }^{*}$ to the fullerene moiety, was efficient. Additionally, the TPA moieties were involved in stabilizing the (zinc porphyrin cation radical)-(fullerene anion radical), $\mathrm{ZnP}^{+\bullet}-\mathrm{C}_{60}{ }^{-\bullet}$, charge-separated state by a charge-dissipation mechanism. ${ }^{115}$ The final lifetime of the charge-separated state was around $1 \mu \mathrm{s}$.

Light harvesting polymer systems utilizing the photoinduced energy and/or electron transfer mechanisms are becoming more and more attractive. ${ }^{116,117}$ Among the different approaches, the donor-acceptor route has received significant attention due to the availability of a broad range of synthetic procedures now available. ${ }^{118-125}$ By appropriate selection of both the donor and acceptor, it is possible to tune the magnitude of the band gap and the absolute energies of the frontier orbitals, as well as the solubility and processability of the resulting polymer making them suitable for photovoltaic applications. In photovoltaic devices, the conjugated polymer film plays one or more crucial roles including light absorption, electron donation, and hole transportation. ${ }^{18-125}$ In the present study, we have exploited the rich spectral and electrochemical properties of both TPA and zinc porphyrin to develop a hybrid polymer. Further, with the use of a supramolecular (via metal-ligand axial coordination) 
approach, ${ }^{93,126-128}$ the $\left[\left(\mathrm{Ph}_{2} \mathrm{~N}\right)_{4} \mathrm{ZnP}\right.$ polymer film]-fullerene donor-acceptor hybrids have been constructed for photoelectrochemical applications. ${ }^{129,130}$

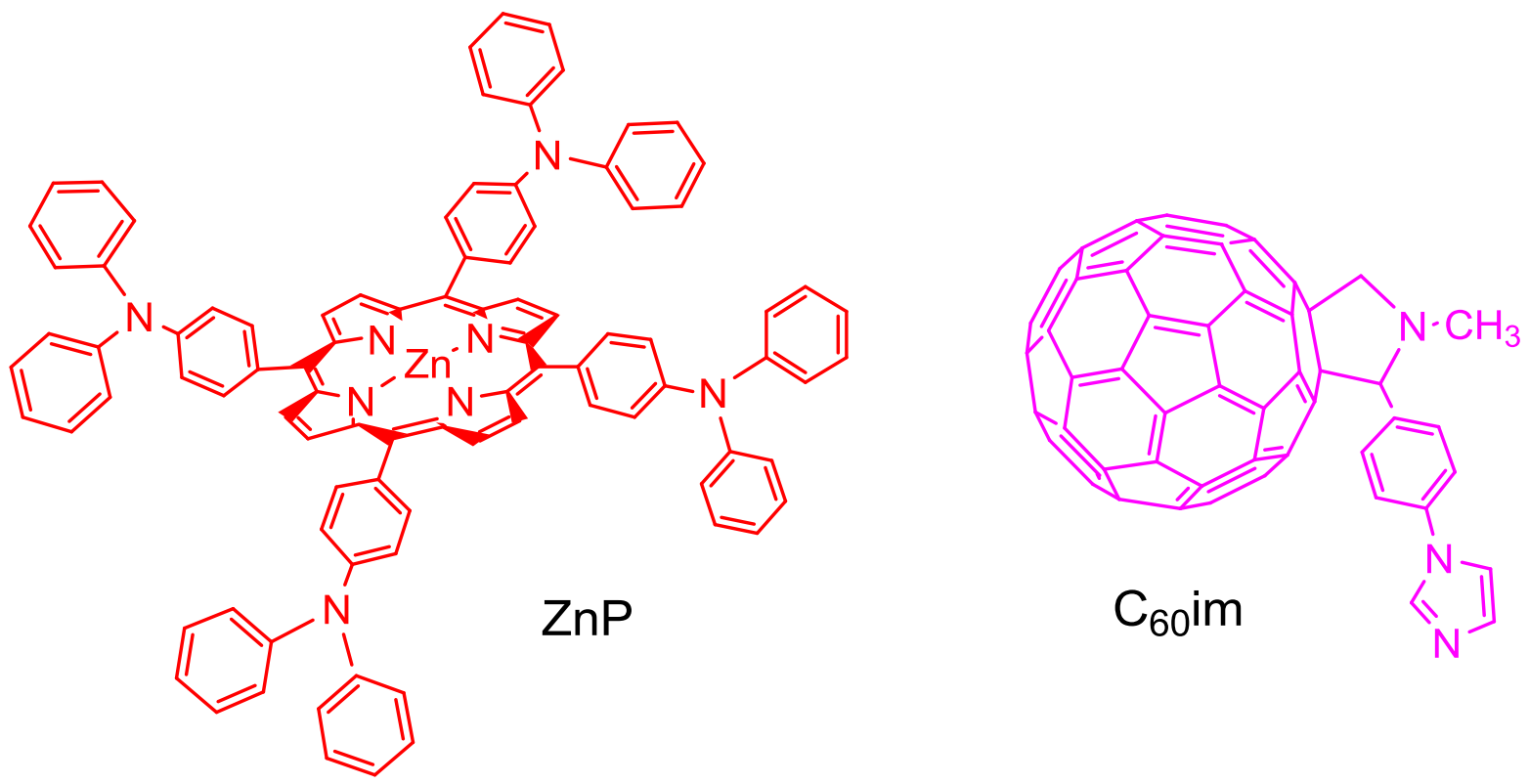

Scheme 3.1: Structural formula of tetrakis(4-(N,N-diphenylamino)phenyl)porphyrinato zinc(II), $\left(\mathrm{Ph}_{2} \mathrm{~N}\right)_{4} \mathrm{ZnP}$, used in forming a $\left(\mathrm{Ph}_{2} \mathrm{~N}\right)_{4} \mathrm{ZnP}$ polymer film electrode via electropolymerization, and imidazole-appended fullerene, C60im, to coordinate the zinc porphyrin in the polymer film to ultimately yield a $\left[\left(\mathrm{Ph}_{2} \mathrm{~N}\right)_{4} \mathrm{ZnP}\right.$ polymer film]-fullerene donor-acceptor hybrid.

To accomplish these tasks, tetrakis(4- $(N, N$ diphenylamino)phenyl)porphyrinatozinc(II), $\left(\mathrm{Ph}_{2} \mathrm{~N}\right)_{4} \mathrm{ZnP}$, was electropolymerized on the $\mathrm{Pt}$ and Au disk as well as ITO surface to obtain electrodes modified with the $\left(\mathrm{Ph}_{2} \mathrm{~N}\right)_{4} \mathrm{ZnP}$ polymer film. These electrodes were subsequently allowed to axially coordinate a $C_{60}$ fullerene derivatized with an imidazole moiety (see Scheme 3.1). This approach resulted in the formation of the electron donor-acceptor hybrid, in which the acceptor was coordinated to the surface-accessible zinc porphyrin sites. That way, the donoracceptor pair was suitably positioned to enable vectorial electron transfer. We have intentionally chosen fullerene ${ }^{131,132}$ as an electron acceptor because of its outstanding electron accepting properties, including favorable reduction potentials ${ }^{133,134}$ and 
relatively low reorganization energy. ${ }^{135-137}$ This newly formed donor-acceptor hybrid material was characterized by the simultaneous cyclic voltammetry (CV) and piezoelectric microgravimetry (PM) measurements using an electrochemical quartz crystal microbalance (EQCM), and by atomic force microscopy (AFM) imaging. Further, the optical absorption and fluorescence studies revealed preserving the $\pi$-electron system of the zinc porphyrin monomer in the polymer film, and quenching of the fluorescence upon binding the fullerene moiety. Photoelectrochemical studies ${ }^{10}$ using the $\left[\left(\mathrm{Ph}_{2} \mathrm{~N}\right)_{4} \mathrm{ZnP}\right.$ polymer film]-fullerene modified ITO electrodes, aided with the $\mathrm{I}_{3}{ }^{-} \mathrm{l}^{-}$ mediating redox couple, revealed cathodic photocurrent generation. Additionally, the incident photon-to-current conversion efficiency (IPCE) of the $\left[\left(\mathrm{Ph}_{2} \mathrm{~N}\right)_{4} \mathrm{ZnP}\right.$ polymer film]-fullerene electrode was higher than that for the film without the coordinated fullerene, thus signifying the importance of the donor-acceptor hybrid and the resulting vectorial electron transfer in light energy conversion.

\subsection{Experiments}

\subsubsection{Electropolymerisation}

Tetrakis(4-(N,N-diphenylamino)phenyl)-porphyrinatozinc(II) ${ }^{112}$ and imidazole derivatized fullerene ${ }^{128}$ were synthesized according to literature procedures. All the solutions purged with argon prior to electrochemical and spectral measurements. All the measurements were performed at ambient temperature $\left(25 \pm 1{ }^{\circ} \mathrm{C}\right)$. Electropolymerization was carried out in a three-electrode glass cell with an ITO, Pt disk or Au/quartz as the working electrode, a Pt foil or wire as the counter electrode and $\mathrm{Ag} / \mathrm{AgCl}$ as the reference electrode in $0.1 \mathrm{M}(\mathrm{TBA}) \mathrm{ClO}_{4}$, in 1,2-dichlorobenzene. The 
number of $\mathrm{CV}$ cycles completed or the potential scan rate controlled thickness of the film. An approximately $0.8 \mathrm{~cm}^{2}$ area of ITO was used for the film formation.

The $\mathrm{C}_{60} \mathrm{im}$ coordinated $\left(\mathrm{Ph}_{2} \mathrm{~N}\right)_{4} \mathrm{ZnP}$ polymer film for photoelectrochemical studies was obtained by soaking the $\mathrm{ITO} /\left[\left(\mathrm{Ph}_{2} \mathrm{~N}\right)_{4} \mathrm{ZnP}\right.$ polymer film] electrode in a 1,2 dichlorobenzene solution of $\mathrm{C}_{60} \mathrm{im}$ for ca. $1 \mathrm{~h}$. The electrode was then rinsed with the 1,2-dichlorobenzene solvent to remove excess of uncoordinated $\mathrm{C}_{60} \mathrm{im}$ and then immediately used for photoelectrochemical studies.

3.3 Results and Discussion

3.3.1 Electropolymerization of Tetrakis(4-(N,N-Diphenylamino)Phenyl)PorphyrinatoZinc(II) and its Axial Coordination of Imidazole-Appended Fullerene to Form a DonorAcceptor Hybrid

Simultaneously recorded with EQCM multi-scan cyclic voltammograms as well as the potential dependence of the resonant frequency change and dynamic resistance change, which led to electropolymeric deposition of the $\left(\mathrm{Ph}_{2} \mathrm{~N}\right)_{4} \mathrm{ZnP}$ polymer film on a quartz resonator, are presented in Figure 3.1. The film deposition was manifested by the decrease of the total resonant frequency, $\Delta f_{\text {total }}$, with the increase of the CV cycle number (Fig.3.1a). This decrease, measured for the mass-loaded quartz resonator immersed in a viscous medium, is caused both by the changes of its mass, $\Delta f_{\text {mass }}$, and visco-elasticity of a contacting medium, $\Delta f_{\text {vis }}$ :

$$
\Delta f_{\text {total }}=\Delta f_{\text {mass }}+\Delta f_{\text {vis }}=-\frac{2 f_{0}^{2} \Delta m}{A\left(\mu_{\mathrm{Q}} \rho_{\mathrm{Q}}\right)^{1 / 2}}+\left(-f_{0}^{3 / 2}\right)\left(\frac{\eta_{\mathrm{L}} \rho_{\mathrm{L}}}{\pi \mu_{\mathrm{Q}} \rho_{\mathrm{Q}}}\right)^{1 / 2}
$$




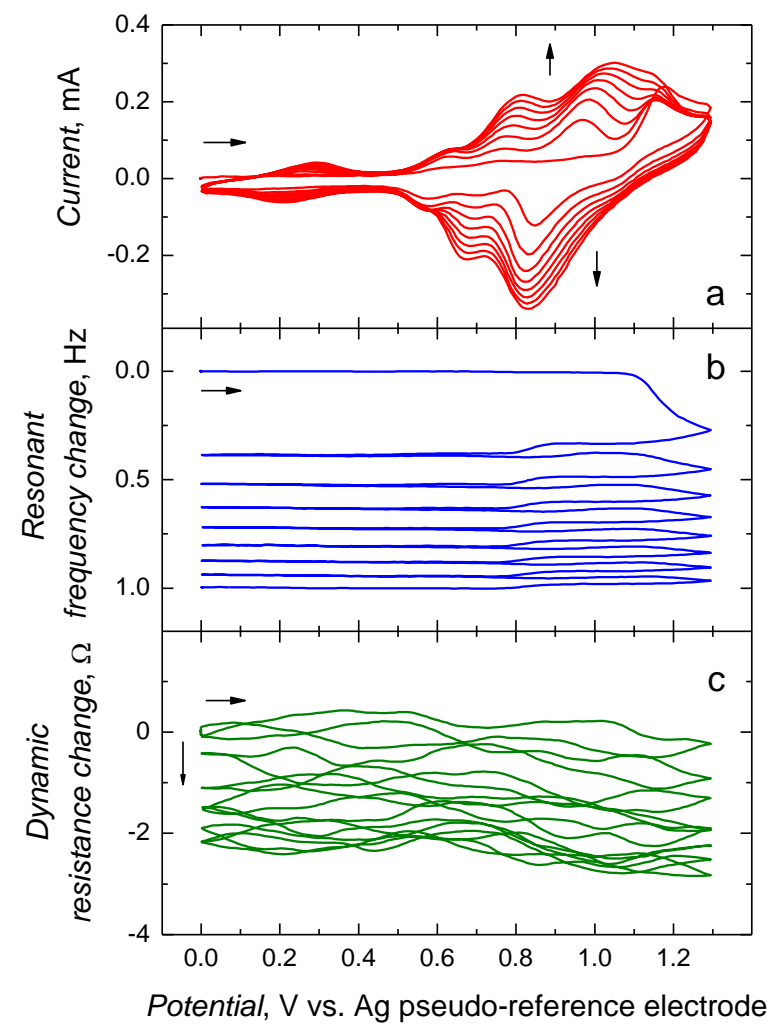

Figure 3.1: Curves of (a) multi-scan cyclic voltammetry as well as the potential dependence of (b) resonant frequency change, and (c) dynamic resistance changes for electropolymerization of a $\left(\mathrm{Ph}_{2} \mathrm{~N}\right)_{4} \mathrm{ZnP}$ polymer film from a 1,2-dichlorobenzene solution of $0.124 \mathrm{mM}$ tetrakis(4-(N,N-diphenylamino)phenyl)porphyrinatozinc(II) and $0.1 \mathrm{M}$ (TBA) $\mathrm{ClO}_{4}$. Potential sweep rate was $100 \mathrm{mV} / \mathrm{sec}$. A $5-\mathrm{mm}$ diameter gold-film electrode of the $10-\mathrm{mHz} \mathrm{Au} / \mathrm{Ti}$ quartz crystal resonator served as the working electrode.

where $\mu_{\mathrm{Q}}=2.947 \times 10^{11} \mathrm{~g} \mathrm{~cm}^{-1} \mathrm{~s}^{-2}$ is the shear modulus of quartz, $\rho_{\mathrm{Q}}=2.648 \mathrm{~g} \mathrm{~cm}^{-3}$ is the quartz density, $\eta\left\llcorner\left(\mathrm{g} \mathrm{cm}^{-1} \mathrm{~s}^{-1}\right)\right.$ and $\rho \mathrm{L}\left(\mathrm{g} \mathrm{cm}^{-3}\right)$ are the dynamic viscosity and density, respectively, of the contacting liquid, $A\left(\mathrm{~cm}^{2}\right)$ is the acoustically active area of a quartz resonator, and $f_{0}(\mathrm{~Hz})$ is the fundamental frequency of the quartz resonator. Qualitatively, considerations involving a quartz resonator in contact with viscous liquid can be extended to cover viscous film coats. Therefore, simultaneous measurement of a variable related to visco-elasticity of a system, like dynamic resistance of the quartz 
resonator $(R)$, was necessary to determine contribution of the visco-elasticity change to the total frequency change:

$$
R=\frac{A}{k^{2}}\left(2 \pi f_{0} \eta_{\mathrm{L}} \rho_{\mathrm{L}}\right)^{1 / 2}
$$

where $k^{2}=7.74 \times 10^{-3}\left(\mathrm{~A}^{2} \mathrm{~s}^{2} \mathrm{~cm}^{-2}\right)$ is the electromechanical coupling factor for the quartz resonator. Hence, from the second component of Equation (1) and Equation (2) it follows that the $\Delta f_{\text {vis }}$ frequency change is opposite to the dynamic resistance:

$$
\Delta f_{\mathrm{vis}}=-\frac{k^{2} R f_{0}}{\pi A\left(2 \mu_{\mathrm{Q}} \rho_{\mathrm{Q}}\right)^{1 / 2}}
$$

The $\Delta f_{\text {total }}$ value for the $\left(\mathrm{Ph}_{2} \mathrm{~N}\right)_{4} \mathrm{ZnP}$ polymer film, measured after six $\mathrm{CV}$ cycles, was 1.3 kHz (Fig.3.1b). The accompanying small changes in the dynamic resistance indicated only minor changes in the film rigidity. Presumably, tight packing of the $\left(\mathrm{Ph}_{2} \mathrm{~N}\right)_{4} \mathrm{ZnP}$ polymer is responsible for this effect. Based on Equation (3) $\Delta f_{\text {vis, }}$ calculated for the $\left(\mathrm{Ph}_{2} \mathrm{~N}\right)_{4} \mathrm{ZnP}$ polymer film, was equal to $\sim 0.2 \mathrm{~Hz}$. This change is negligibly small comparing to that of $\Delta f_{\text {total }}$. It means that the film coating the quartz resonator surface remains rigid during deposition and the change of its viscosity insignificantly influences the total frequency change. Moreover, visco-elasticity of the film stays virtually unchanged during each CV cycle like if it was intact to the counterion ingress and egress from the film during its charging and discharging. After subtraction of $\Delta f_{\text {vis }}$ from $\Delta f_{\text {total }}$, based on the first part of Equation (1) the mass of the deposited $\left(\mathrm{Ph}_{2} \mathrm{~N}\right)_{4} \mathrm{ZnP}$ polymer film was calculated as $(863 \pm 1) \mathrm{ng}$.

The quartz resonator, with its electrode coated with the $\left(\mathrm{Ph}_{2} \mathrm{~N}\right)_{4} \mathrm{ZnP}$ polymer film, was mounted in a flow-through EQCM 5610 holder and the resonant frequency changes accompanying injections of the $\mathrm{C}_{60} \mathrm{im}$ in 1,2-dichlorobenzene samples were recorded. 
Figure 3.2a shows a frequency response to the initial three consecutive injections. After each injection, frequency decreased and reached a plateau rather than formed a peak clearly indicating desirable irreversible $\mathrm{C}_{60} \mathrm{im}$ binding to the $\left(\mathrm{Ph}_{2} \mathrm{~N}\right)_{4} \mathrm{ZnP}$ polymer. With the use of the Sauerbrey equation ${ }^{100,138}$

$$
\Delta f_{\text {mass }}=-\frac{2 f_{0} \Delta m}{A\left(\mu_{\mathrm{Q}} \rho_{\mathrm{Q}}\right)^{1 / 2}}
$$

the frequency changes were converted into the mass changes. A total frequency change was $141 \mathrm{~Hz}$ corresponding to $122 \mathrm{ng}$ of $\mathrm{C}_{60} \mathrm{im}$ immobilized on the $\left(\mathrm{Ph}_{2} \mathrm{~N}\right)_{4} \mathrm{ZnP}$ polymer film after three injections. This value corresponds to $\sim 2.1$ monolayers of $\mathrm{C}_{60} \mathrm{im}$ at hexagonal packing. Most likely, the fullerene ligand only slightly penetrates the polymer film ligating the outermost ZnP sites exposed to solution.
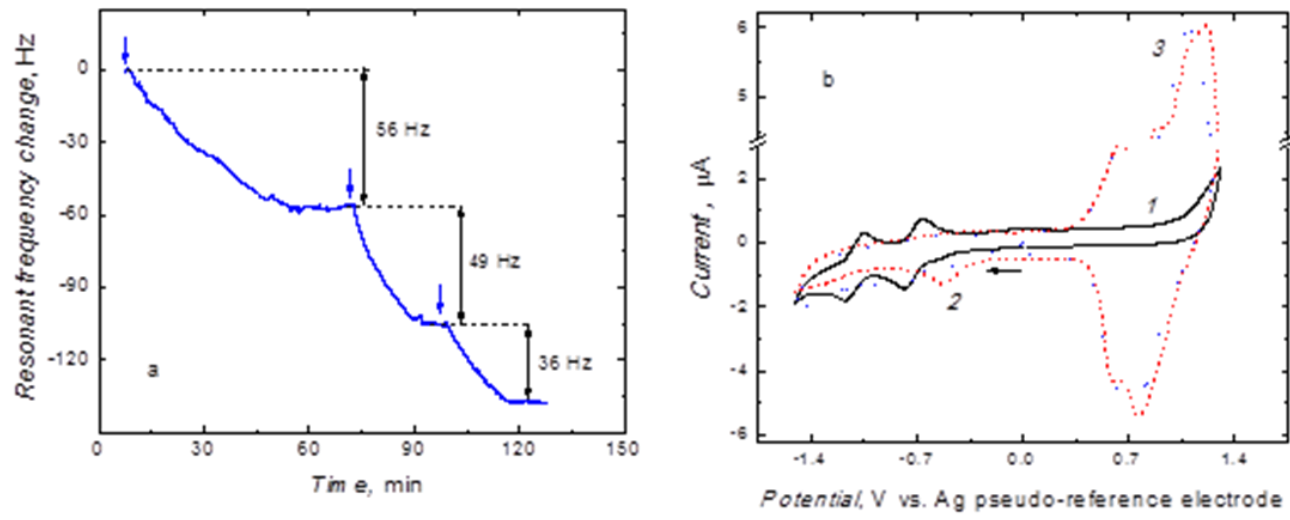

Figure 3.2: (a) Flow-injection analysis measurements with the EQCM detection of the $\mathrm{C}_{60} \mathrm{im}$ binding to the $\left(\mathrm{Ph}_{2} \mathrm{~N}\right)_{4} \mathrm{ZnP}$ polymer film to form the donor-acceptor dyad. Sample volume of each injection of $17.4 \mathrm{mM} \mathrm{C60im} \mathrm{in} \mathrm{1,2-dichlorobenzene} \mathrm{was} 1 \mathrm{~mL}$. Flow rate was $50 \mu \mathrm{L} / \mathrm{min}$. The frequency change accompanying initial three consecutive injections are shown. (b) Cyclic voltammograms at the 1.6- $\mathrm{mm}$ diameter Au disk electrode for (1) $0.5 \mathrm{mM} \mathrm{C60im,} \mathrm{(2)}\left(\mathrm{Ph}_{2} \mathrm{~N}\right)_{4} \mathrm{ZnP}$ polymer film and (3) C60im bound to the $\left(\mathrm{Ph}_{2} \mathrm{~N}\right)_{4} \mathrm{ZnP}$ polymer film demonstrating formation of an electro-active $\left[\left(\mathrm{Ph}_{2} \mathrm{~N}\right)_{4} \mathrm{ZnP}\right.$ polymer film]-C60im donor-acceptor dyad in $0.1 \mathrm{M}(\mathrm{TBA}) \mathrm{ClO}_{4}$, in 1,2-dichlorobenzene. Potential scan rate was $100 \mathrm{mV} / \mathrm{s}$. 
CV studies of the $\left[\left(\mathrm{Ph}_{2} \mathrm{~N}\right)_{4} \mathrm{ZnP}\right.$ polymer film]-fullerene modified electrode confirmed the presence of the coordinated fullerene (Figure 3.2b). The voltammogram for the $\left[\left(\mathrm{Ph}_{2} \mathrm{~N}\right)_{4} \mathrm{ZnP}\right.$ polymer film]-fullerene modified electrode (Figure 3.2b) revealed cathodic peaks at -0.70 and $-1.30 \mathrm{~V}$ being close to those for $\mathrm{C}_{60}$ im in solution. ${ }^{93,126-128}$ However, the peak currents were directly proportional to the potential scan rate, as expected for a surface-confined redox couple. ${ }^{9}$ In a control cyclic voltammogram, recorded for the $\left(\mathrm{Ph}_{2} \mathrm{~N}\right)_{4} \mathrm{ZnP}$ polymer film without $\mathrm{C}_{60}$ im (Curve 2 in Figure.3.2b), no such cathodic peaks were observed.
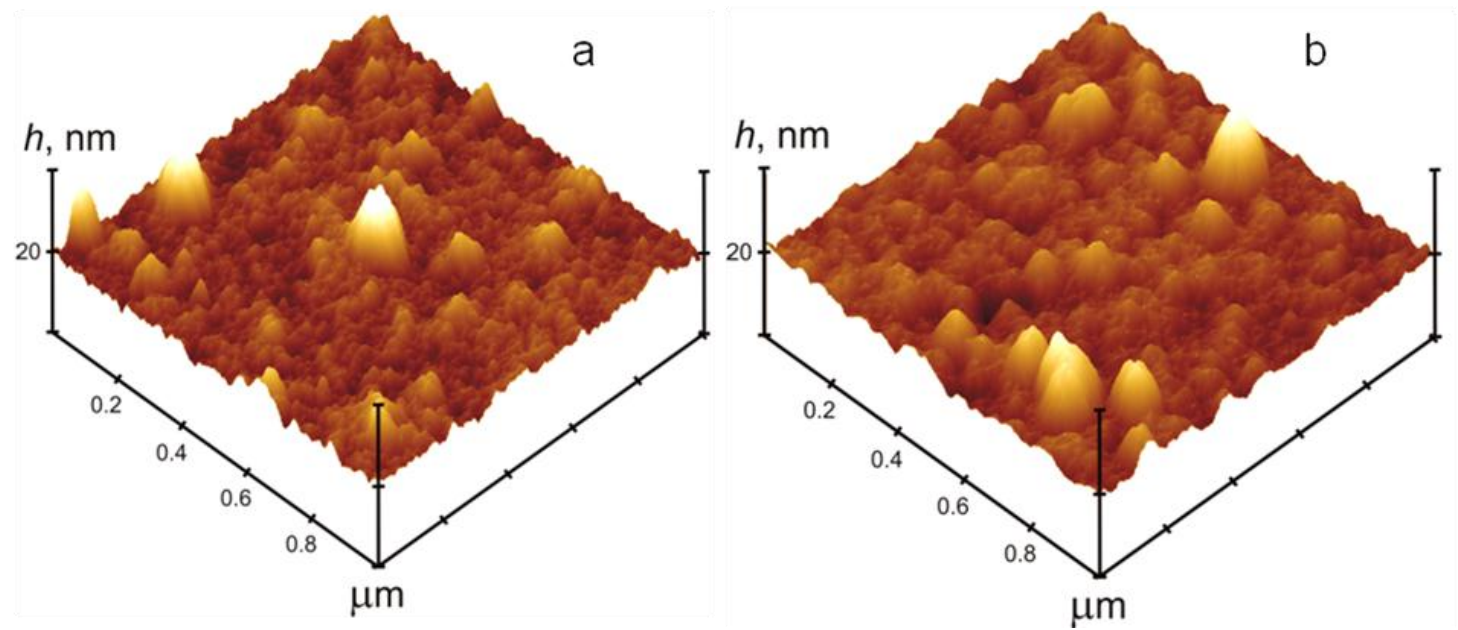

Figure 3.3: Atomic force microscopy tapping mode $1 \times 1 \mu \mathrm{m}$ image of (a) the $\left(\mathrm{Ph}_{2} \mathrm{~N}\right)_{4} \mathrm{ZnP}$ polymer film and (b) the $\left(\mathrm{Ph}_{2} \mathrm{~N}\right)_{4} \mathrm{ZnP}$ polymer film with the bound $\mathrm{C}_{60} \mathrm{im}$ ligand, deposited by electropolymerization on an Au/Cr-coated glass slide.

The AFM-imaged $\left(\mathrm{Ph}_{2} \mathrm{~N}\right)_{4} \mathrm{ZnP}$ polymer topography in the absence and presence of the coordinated $\mathrm{C}_{60} \mathrm{im}$ ligand is shown in Figure $3.3 \mathrm{a}$ and $3.3 \mathrm{~b}$, respectively. The $\left(\mathrm{Ph}_{2} \mathrm{~N}\right)_{4} \mathrm{ZnP}$ polymer film is uniform with microscopically distinguishable $150-200 \mathrm{~nm}$ clusters. Average thickness of this film prepared from the $0.12 \mathrm{mM}\left(\mathrm{Ph}_{2} \mathrm{~N}\right)_{4} \mathrm{ZnP}$ in $0.1 \mathrm{M}$ (TBA) $\mathrm{ClO}_{4}$ in 1,2-dichlorobenzene, in the course of $10 \mathrm{CV}$ cycles at $0.1 \mathrm{~V} / \mathrm{s}$, in the potential range 0 to $1.30 \mathrm{~V}$, was $(43 \pm 2) \mathrm{nm}$. Upon binding $\mathrm{C}_{60} \mathrm{im}$, the film morphology 
was not significantly changed (Fig. 3.3b), however, the image was more diffuse compared to that of the $\left(\mathrm{Ph}_{2} \mathrm{~N}\right)_{4} \mathrm{ZnP}$ polymer film alone. The roughness factor, $R_{\mathrm{sa}}$, for surface of the $\left(\mathrm{Ph}_{2} \mathrm{~N}\right)_{4} \mathrm{ZnP}$ polymer film before and after $\mathrm{C}_{60} \mathrm{im}$ binding was 1.010 and 1.022, respectively.

3.3.2 Optical Absorptions and Emission Behavior of the $\left(\mathrm{Ph}_{2} \mathrm{~N}\right)_{4} \mathrm{ZnP}$ Polymer Film]Fullerene Donor-Acceptor Hybrid

Figure 3.4a shows optical absorption spectrum for the $\left(\mathrm{Ph}_{2} \mathrm{~N}\right)_{4} \mathrm{ZnP}$ polymer film coated ITO electrode before and after $\mathrm{C}_{60}$ im binding. In the absence of $\mathrm{C}_{60} \mathrm{im}$, the $\left(\mathrm{Ph}_{2} \mathrm{~N}\right)_{4} \mathrm{ZnP}$ polymer film peaks at 443,565 , and $610 \mathrm{~nm}$ are seen (Trace 1 in Figure 3.4a). These peaks were broader than those for monomeric $\left(\mathrm{Ph}_{2} \mathrm{~N}\right)_{4} \mathrm{ZnP}$ used for electropolymerization (see Figure 3.5a for the spectrum for the $\left(\mathrm{Ph}_{2} \mathrm{~N}\right)_{4} \mathrm{ZnP}$ monomer in 1,2-dichlorobenzene). Upon binding $\mathrm{C}_{60} \mathrm{im}$, the entire spectrum was red shifted by 3-4 $\mathrm{nm}$ (Trace 2 in Figure 3.4a) typical of that induced by axial coordination of a nitrogenous base to the zinc central metal atom of porphyrin. ${ }^{4,39-}$ ${ }^{41}$ In addition, intensity of the peak at $310 \mathrm{~nm}$ increased due to the presence of fulleropyrrolidine. Importantly, the presence of the Soret and $Q$ bands of $\left(\mathrm{Ph}_{2} \mathrm{~N}\right)_{4} \mathrm{ZnP}$ indicates that the zinc porphyrin $\pi$-electron system is intact, i.e., it is not destroyed during electrochemical polymerization. Moreover, it is evident from these spectroscopic data that the peripheral TPA substituents and not the porphyrin ring participate in the electropolymerization. ${ }^{21}$

The steady-state fluorescence spectrum for the $\left(\mathrm{Ph}_{2} \mathrm{~N}\right)_{4} \mathrm{ZnP}$ polymer film also supports the polymer retaining its genuine porphyrin $\pi$-electron system. Two weak emission bands, located at 613 and $678 \mathrm{~nm}$ in the spectrum, (Trace 1' in Figure 3.4b) 
compare well with the emission bands for the $\left(\mathrm{Ph}_{2} \mathrm{~N}\right)_{4} \mathrm{ZnP}$ monomer in solution located at 623 and 662(sh) nm (see Figure 3.5a and 3.5b for optical absorption and emission spectrum for the $\left(\mathrm{Ph}_{2} \mathrm{~N}\right)_{4} \mathrm{ZnP}$ monomer). Coordination of $\mathrm{C}_{60} \mathrm{im}$ results in quenching of both bands (Trace 2 in Figure 3.4b) indicating occurrence of some excited-state
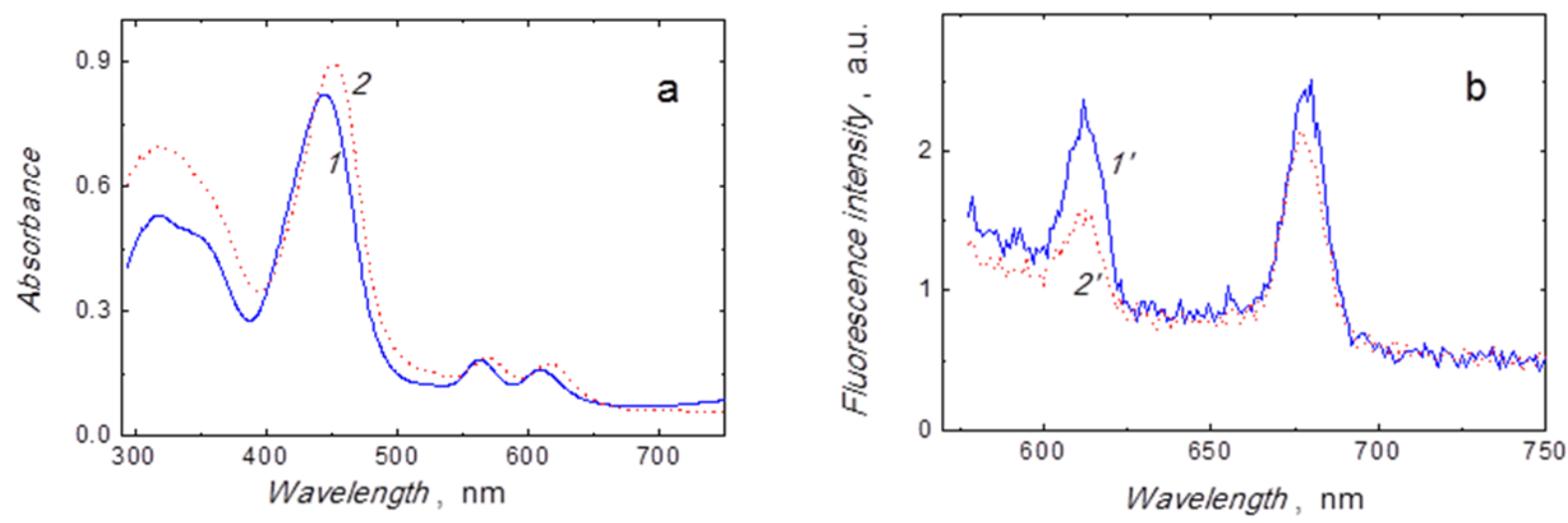

Figure 3.4: Spectra of (a) optical absorption and (b) fluorescence for the $\left(\mathrm{Ph}_{2} \mathrm{~N}\right)_{4} \mathrm{ZnP}$ polymer film deposited on the ITO electrode (1 and 1') before and (2 and 2') after coordinating $\mathrm{C}_{60} \mathrm{im}$. $\lambda_{\mathrm{ex}}=444 \mathrm{~nm}$
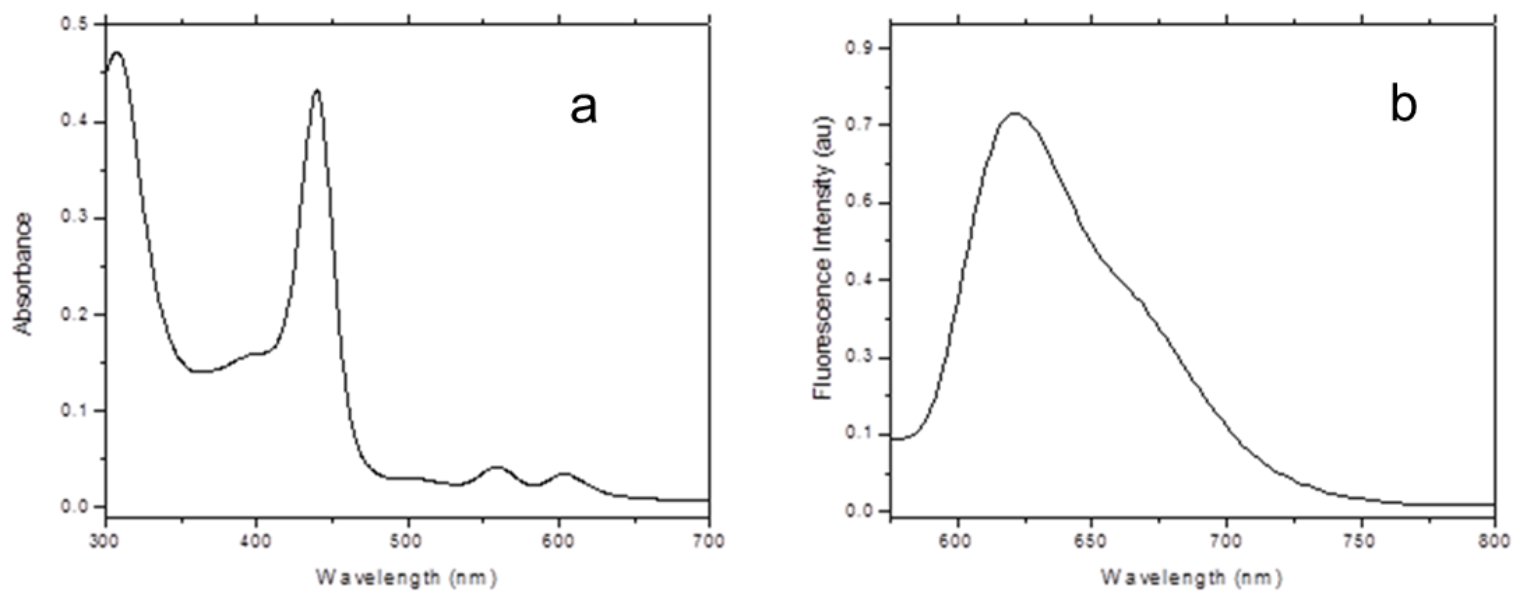

Figure 3.5: (a) Absorption spectrum of $\left(\mathrm{Ph}_{2} \mathrm{~N}\right)_{4} \mathrm{ZnP}$ in o-dichlorobenzene. (b) Emission spectrum of $\left(\mathrm{Ph}_{2} \mathrm{~N}\right)_{4} \mathrm{ZnP}$ in O-dichlorobenzene. $\lambda_{\mathrm{ex}}=560 \mathrm{~nm}$.

photochemical events. In the absence of any emission corresponding to $\mathrm{C}_{60} \mathrm{im}$ in the 700-750 nm range, energy transfer as a quenching mechanism could be ruled out. 
Therefore, based on energetic considerations, ${ }^{139}$ electron transfer is postulated to account for this mechanism.

3.3.3 Photoelectrochemical Studies of the $\left(\mathrm{Ph}_{2} \mathrm{~N}\right)_{4} \mathrm{ZnP}$ Polymer Film]-Fullerene DonorAcceptor Hybrid

Quenching of the electron transfer in the $\left[\left(\mathrm{Ph}_{2} \mathrm{~N}\right)_{4} \mathrm{ZnP}\right.$ polymer film]-fullerene hybrid, properly positioned for vectorial electron transfer, prompted us to investigate photoelectrochemical properties of the hybrid. Herein, three issues were mainly addressed, viz., (i) direction of the photocurrent flow in the photoelectrochemical cell, (ii) influence of the relative thickness of the $\left(\mathrm{Ph}_{2} \mathrm{~N}\right)_{4} \mathrm{ZnP}$ polymer film on the photovoltage and photocurrent generation, and (iii) the role of coordinated $\mathrm{C}_{60} \mathrm{im}$ in improving the light-to-energy conversion efficiency.

Conventional (organic dye)-sensitized ITO electrodes produce anodic photocurrents in the presence of the $\mathrm{I}_{3}^{-} / \mathrm{Il}^{-}$mediating redox couple. ${ }^{10,140-143}$ In this system, the general photoelectrochemical mechanism involves electron injection from a dye in its excited state into the photoelectrode conduction band, with subsequent neutralization of the cationic dye by the iodide mediator. In result, anodic photocurrent flows. Alternatively, cathodic photocurrent can be generated in a dye-sensitized photocell made of the ITO/porous multilayer porphyrinic molecular squares. ${ }^{144}$ In this photoelectrochemical cell, the mechanism of cathodic photocurrent generation involves the porphyrin-excited state quenching with the $\mathrm{I}_{3}{ }^{-}$mediator to produce an oxidized dye. Because of a ground-state donor (chromophore) - acceptor $\left(\mathrm{I}_{3}^{-}\right.$mediator) complex formation, electron transfer was possible despite the short excited-state lifetime of porphyrin. Following quenching, the oxidizing equivalent is delivered to the ITO 
electrode via a ground-state redox electron hopping through the multilayer porphyrin film. ${ }^{144}$ Such a mechanism also seems to occur in the currently investigated system of the ITO/[(Ph $\left.{ }_{2} \mathrm{~N}\right)_{4} \mathrm{ZnP}$ polymer film] and the $\mathrm{I}_{3}{ }^{-} / \mathrm{I}^{-}$mediating couple. ${ }^{145,146}$

Figure 3.6 shows the photocurrent-photovoltage characteristics recorded for the ITO/ $\left(\mathrm{Ph}_{2} \mathrm{~N}\right)_{4} \mathrm{ZnP}$ polymer film electrode, in the acetonitrile solution, for different $\mathrm{I}^{-}$-to- $\mathrm{I}_{2}$ concentration ratios. In the presence of only $\mathrm{I}^{-}$or small amounts of $\mathrm{I}_{3}^{-}$, the photocurrent was low. When the concentration of $I_{3}^{-}$was gradually increased by addition of $I_{2}$, the photocurrent increased at lower photovoltages. The photocurrent was appreciably high when analytical concentration of $\mathrm{I}^{-}$and $\mathrm{I}_{2}$ was $0.1 \mathrm{M}$ and $0.119 \mathrm{M}$, respectively, resulting in the $\mathrm{I}_{3}{ }^{-}$concentration of $0.119 \mathrm{M}$. The $\mathrm{I}_{3}{ }^{-}$concentration was calculated from the



Figure 3.6: Photocurrent-photovoltage characteristics for the $\left(\mathrm{Ph}_{2} \mathrm{~N}\right)_{4} \mathrm{ZnP}$ polymer film coated ITO electrode (in the absence of $\mathrm{C}_{60}$ ) in acetonitrile solutions, which were: (1) 92, (2) 96 , (3) 101 , (4) 105 , (5) 110 , and (6) $119 \mathrm{mM}$ in I 2 and $0.1 \mathrm{M}$ in iodide stability constant of $\sim 107 \mathrm{M}-1$ at room temperature of the 13- complex, whose equilibrium describes Equation (5). ${ }^{145}$

$$
\mathrm{I}^{-}+\mathrm{I}_{2} \rightleftharpoons \mathrm{I}^{-}
$$

The photocurrent increase and photovoltage decrease caused by the $\mathrm{I}_{3}{ }^{-}$ concentration increase is in agreement with the cathodic photovoltaic effect. It is likely 
that the $\mathrm{I}_{3}{ }^{-}$electron acceptor forms a ground-state complex with the $\left(\mathrm{Ph}_{2} \mathrm{~N}\right)_{4} \mathrm{ZnP}$ moiety and oxidatively quenches the excited state, as shown below by Equations (6) and (7).

$$
\begin{aligned}
& \left(\mathrm{Ph}_{2} \mathrm{~N}\right)_{4} \mathrm{ZnP}+\mathrm{I}_{3}^{-}=\left[\left(\mathrm{Ph}_{2} \mathrm{~N}\right)_{4} \mathrm{ZnP}: \mathrm{I}_{3}{ }^{-}\right] \\
& {\left[\left(\mathrm{Ph}_{2} \mathrm{~N}\right)_{4} \mathrm{ZnP}: \mathrm{I}_{3}^{-}\right]+h v \rightarrow\left(\mathrm{Ph}_{2} \mathrm{~N}\right)_{4} \mathrm{ZnP}^{+\bullet}+\mathrm{I}_{3}{ }^{2-} \rightarrow \text { Products }(7)}
\end{aligned}
$$

Here, (i) the electron from $\left[{ }^{1}\left(\mathrm{Ph}_{2} \mathrm{~N}\right)_{4} \mathrm{ZnP}^{*}: I_{3}\right]$ is transported by the $\mathrm{I}^{-}$mediator through the solution to the $\mathrm{Pt}$ counter electrode, (ii) the $\left(\mathrm{Ph}_{2} \mathrm{~N}\right)_{4} \mathrm{ZnP}$-localized hole travels through the redox conducting polymer film until it reaches the ITO surface, and (iii) the electron from the counter electrode moves through the external circuit, reaches the ITO photocathode and reacts with the $\left(\mathrm{Ph}_{2} \mathrm{~N}\right)_{4} \mathrm{ZnP}^{+\bullet}$ at the $\mathrm{ITO} /\left[\left(\mathrm{Ph}_{2} \mathrm{~N}\right)_{4} \mathrm{ZnP}\right.$ polymer film] interface to regenerate the light-absorbing neutral form of the $\left(\mathrm{Ph}_{2} \mathrm{~N}\right)_{4} \mathrm{ZnP}$ polymer.

Having established a suitable $\mathrm{I}^{-} / \mathrm{l}_{2}$ concentration ratio of $1.0 \mathrm{mM}: 1.0 \mathrm{mM}$ for optimum cathodic photocurrent generation, we next focused on visualizing the effect of the relative $\left(\mathrm{Ph}_{2} \mathrm{~N}\right)_{4} \mathrm{ZnP}$ polymer film thickness on the photocurrent generation. For this, $\left(\mathrm{Ph}_{2} \mathrm{~N}\right)_{4} \mathrm{ZnP}$ polymer film of different thickness was deposited onto the ITO electrodes by varying either the number of $\mathrm{CV}$ cycles or the potential scan rate. Optical absorption spectra were then recorded to estimate the relative concentration of zinc porphyrin in the polymer (Figure 3.7).

Figure 3.8 shows the photocurrent-photovoltage characteristics for the ITO electrode coated with the $\left(\mathrm{Ph}_{2} \mathrm{~N}\right)_{4} \mathrm{ZnP}$ polymer film of different thickness prepared at different potential scan rates. Clearly, thinner $\left(\mathrm{Ph}_{2} \mathrm{~N}\right)_{4} \mathrm{ZnP}$ polymer films are preferred for better photocell performance, a result that well agrees with those in literature where 
the multilayer thick films were less effective than the few-layer or monolayer thin films. $^{23,147}$

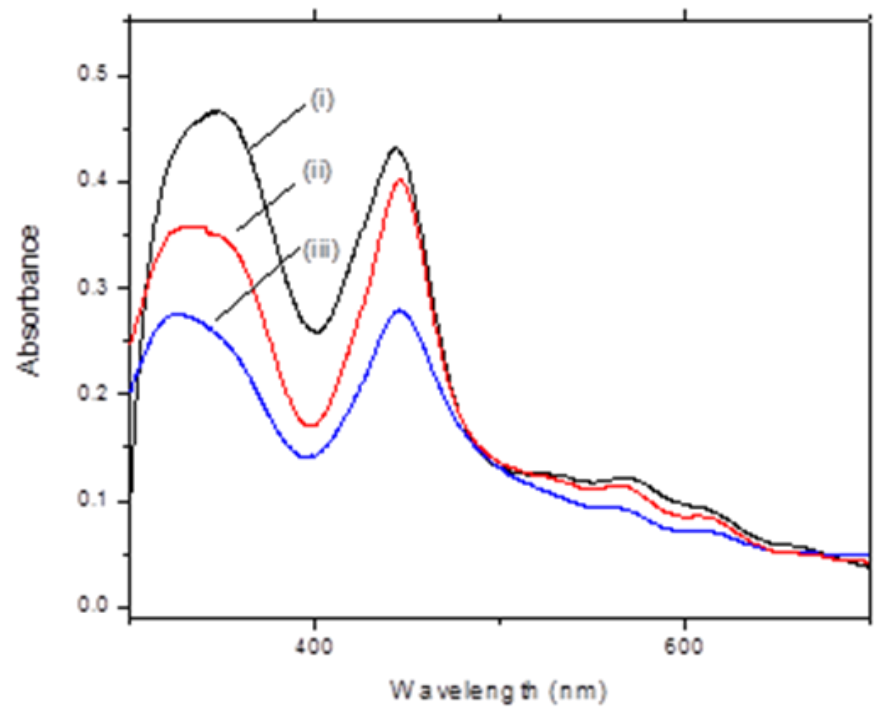

Figure 3.7: Absorbance spectrum of $\left(\mathrm{Ph}_{2} \mathrm{~N}\right)_{4} \mathrm{ZnP}$ polymer film on ITO electrode obtained under different scan rates; (i) $25 \mathrm{mV} / \mathrm{s}$, (ii) $50 \mathrm{mV} / \mathrm{s}$ and (iii) $75 \mathrm{mV} / \mathrm{s}$ in odichlorobenzene containing $0.1 \mathrm{M}(\mathrm{TBA}) \mathrm{ClO}_{4}$.

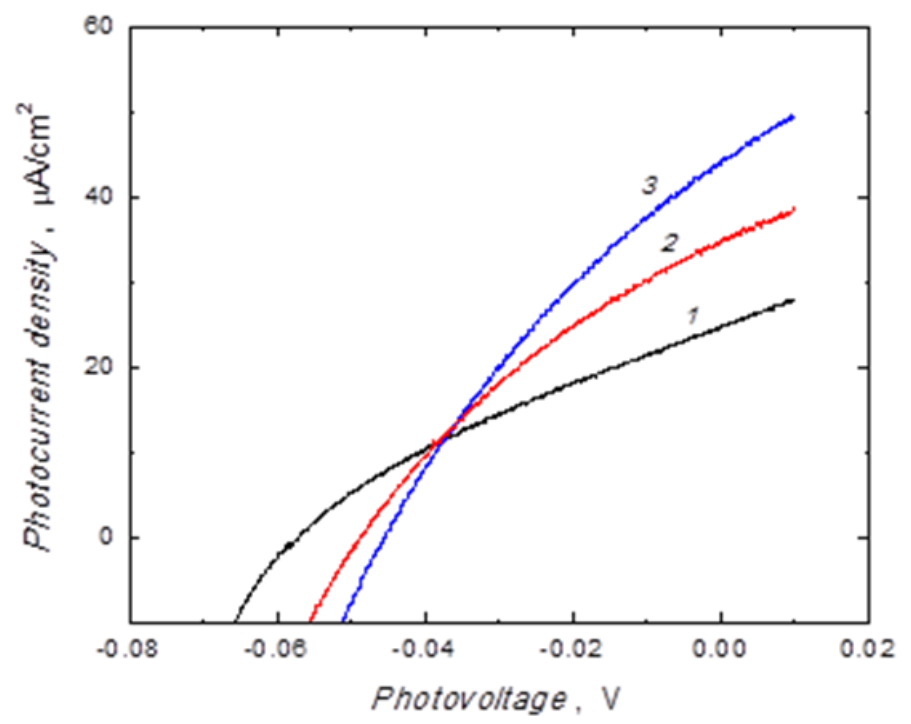

Figure 3.8: Photocurrent-photovoltage characteristics for electrodes modified with the $\left(\mathrm{Ph}_{2} \mathrm{~N}\right)_{4} \mathrm{ZnP}$ polymer film of different thickness, prepared by scanning the potential at (1) 25 , (2) 50 , and (3) $75 \mathrm{mV} / \mathrm{s}$ during film deposition (10 CV cycles at $25 \mathrm{mV} / \mathrm{s}$ ). The photocurrent-photovoltage curves were generated for the acetonitrile solution of the $\mathrm{I}_{3}$ / $\mathrm{I}^{-}(0.1 \mathrm{M} / 0.1 \mathrm{M})$ mediating redox couple. 
Open-circuit potential is lower and short-circuit current is slightly higher for the $\left(\mathrm{Ph}_{2} \mathrm{~N}\right)_{4} \mathrm{ZnP}$ polymer film deposited at the potential scan rate of $50 \mathrm{mV} / \mathrm{s}$ and higher. These results also support the photocurrent generation mechanism discussed above. That is, minimizing the hole transport distance through the film enhances the overall photovoltaic behavior.

Finally, the role of the fullerene, coordinated to the $\left(\mathrm{Ph}_{2} \mathrm{~N}\right)_{4} \mathrm{ZnP}$ polymer film in the photovoltaic behavior was investigated. For these studies, the $\left(\mathrm{Ph}_{2} \mathrm{~N}\right)_{4} \mathrm{ZnP}$ polymer film characteristics and the $\mathrm{I}^{-} / \mathrm{I}_{3}{ }^{-}$concentration were optimized based on the studies described above. Figure 3.9 shows the effect of light on photovoltaic performance of the electrode modified either with the $\left(\mathrm{Ph}_{2} \mathrm{~N}\right)_{4} \mathrm{ZnP}$ polymer film alone, or the $\left(\mathrm{Ph}_{2} \mathrm{~N}\right)_{4} \mathrm{ZnP}$ polymer film with the coordinated $\mathrm{C}_{60} \mathrm{im}$ ligand. Both the photocurrent and photovoltage generation was examined in a light-switching experiment. The photocurrents increased as much as three times while the photovoltage doubled in the presence of the coordinated fullerene. Moreover, these modulations were reversible with respect to both the amplitude of photocurrent and photovoltage. $I-V$ characteristics shown in the Figure 3.10 also demonstrate the significance of the coordinated $\mathrm{C}_{60} \mathrm{im}$ in enhancing the photoelectrochemical performance. The monochromatic IPCE, defined as the number of electrons generated by light in the outer circuit divided by the number of incident photons, ${ }^{148,149}$ was determined according to Equation (8)

$$
\operatorname{IPCE}(\%)=100 \times 1240 \times I_{\mathrm{sC}}\left(\mathrm{mA} \mathrm{cm} \mathrm{cm}^{-2}\right) /\left[\lambda(\mathrm{nm}) \times P_{\mathrm{in}}\left(\mathrm{mW} \mathrm{cm}^{-2}\right)\right]
$$

where $I_{\mathrm{sc}}$ is the short-circuit photocurrent generated by the incident monochromatic light and $\lambda$ is the wavelength of this light with intensity $P_{\text {in }}$. 


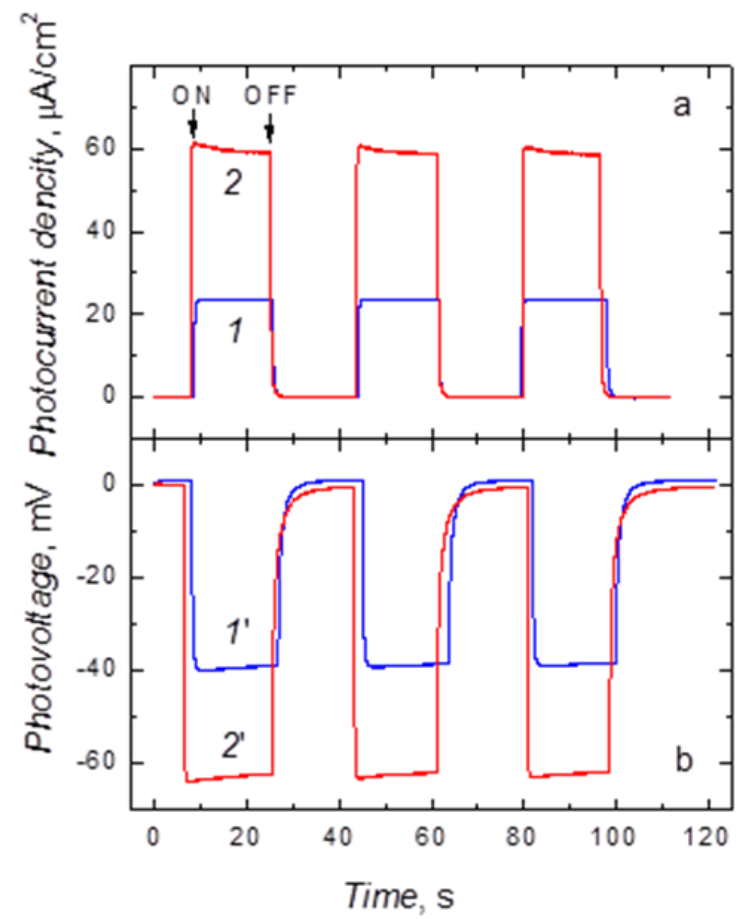

Figure 3.9: Effect of light switching on (a) photocurrent and (b) photovoltage of the $\left(\mathrm{Ph}_{2} \mathrm{~N}\right)_{4} \mathrm{ZnP}$ polymer film coated ITO electrode in the (1 and 1') absence and (2 and 2') presence of $\mathrm{C}_{60} \mathrm{im}$. The photocurrent and photovoltage switching were recorded in acetonitrile solution containing $\mathrm{I}_{3}^{-} / \mathrm{I}^{-}(0.1 \mathrm{M} / 0.1 \mathrm{M})$ mediating redox couple

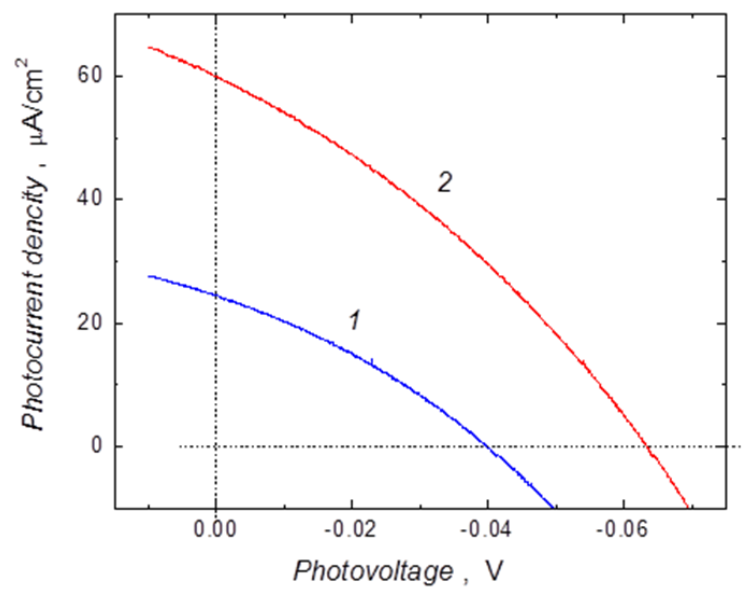

Figure 3.10: Photocurrent-photovoltage (I-V) characteristics for electrodes modified with the $\left(\mathrm{Ph}_{2} \mathrm{~N}\right)_{4} \mathrm{ZnP}$ polymer film (1) in the absence and (2) presence of $\mathrm{C}_{60} \mathrm{im}$. The $I-V$ curves were generated for the acetonitrile solution of the $\mathrm{I}_{3}{ }^{-} / \mathrm{I}^{-}(0.1 \mathrm{M} / 0.1 \mathrm{M})$ mediating redox couple. 
The photocurrent action spectrum of the $\left(\mathrm{Ph}_{2} \mathrm{~N}\right)_{4} \mathrm{ZnP}$ polymer film modified electrode in the absence and presence of coordinated $\mathrm{C}_{60} \mathrm{im}$, in a mediator solution of $0.1 \mathrm{M}(\mathrm{TBA}) \mathrm{I}$ and $0.1 \mathrm{M} \mathrm{I}_{2}$, in acetonitrile, with a Pt foil as the counter electrode, is shown in Figure 3.11a. Both these spectra resemble those of the absorption spectra shown in Figure 3.4a. However, photocurrents in the action spectrum, recorded in the presence of coordinated $\mathrm{C}_{60} \mathrm{im}$, are enhanced again signifying the importance of the coordinated fullerene. At the wavelength of maximum photocurrent, the IPCE was calculated as $\sim 2 \%$ for the $\left[\left(\mathrm{Ph}_{2} \mathrm{~N}\right)_{4} \mathrm{ZnP}\right.$ polymer film]-fullerene modified electrode.

Figure $3.11 \mathrm{~b}$ schematically illustrates the mechanism of photocurrent generation in the $\left[\left(\mathrm{Ph}_{2} \mathrm{~N}\right)_{4} \mathrm{ZnP}\right.$ polymer film]-fullerene modified electrode. As documented by the photochemical studies, photoexcitation of the $\left(\mathrm{Ph}_{2} \mathrm{~N}\right)_{4} \mathrm{ZnP}$ moiety in the donor-acceptor hybrid results in vectorial photoinduced electron transfer to generate radical ion pair, $\left(\mathrm{Ph}_{2} \mathrm{~N}\right)_{4} \mathrm{ZnP}^{+\bullet}-\mathrm{C}_{60}{ }^{-}$. The resulting $\mathrm{C}_{60^{-}}$transfers electron to the $\mathrm{I}^{-} / \mathrm{I}_{3}^{-}$mediating couple to close the circuit. The $\left(\mathrm{Ph}_{2} \mathrm{~N}\right)_{4} \mathrm{ZnP}$-localized hole travels through the conducting $\left(\mathrm{Ph}_{2} \mathrm{~N}\right)_{4} \mathrm{ZnP}$ polymer film until it reaches the ITO surface while the electron from the counter Pt electrode flows through the external circuit and, when reaching the photocathode, it neutralizes $\left(\mathrm{Ph}_{2} \mathrm{~N}\right)_{4} \mathrm{ZnP}^{+\bullet}$ in the polymer film.

The role of the coordinated fullerene in enhancement of the photocurrent and photovoltage generation is borne out from the present study. In the absence of fullerene, the excited $\left(\mathrm{Ph}_{2} \mathrm{~N}\right)_{4} \mathrm{ZnP}$ moiety donates electron within its excited state lifetime of $2.4 \mathrm{~ns}$ to the intermolecularly mediating $\mathrm{I}_{3}$. However, coordination of $\mathrm{C}_{60} \mathrm{im}$ to the $\left(\mathrm{Ph}_{2} \mathrm{~N}\right)_{4} \mathrm{ZnP}$ moiety in the polymer film results in the formation of the $\left(\mathrm{Ph}_{2} \mathrm{~N}\right)_{4} \mathrm{ZnP^{+ \bullet }}$ $\mathrm{C}_{60}{ }^{-\bullet}$ radical ion pair with lifetime in the microsecond range ${ }^{115}$ thus providing sufficient 
time for the charge transfer from $\mathrm{C}_{60}{ }^{-\bullet}$ to $\mathrm{I}_{3}^{-}$. This ion-pair formation eventually improves the photovoltaic response both in terms of the photocurrent and photovoltage generation.

(a)

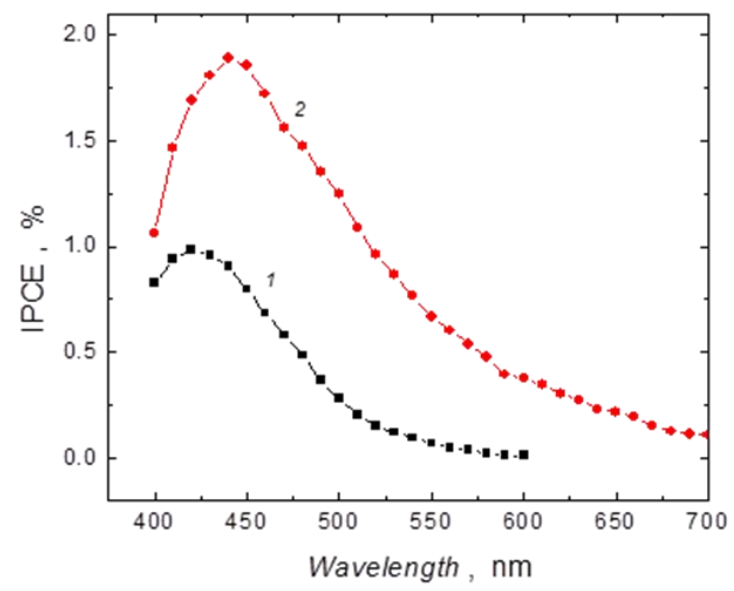

(b)

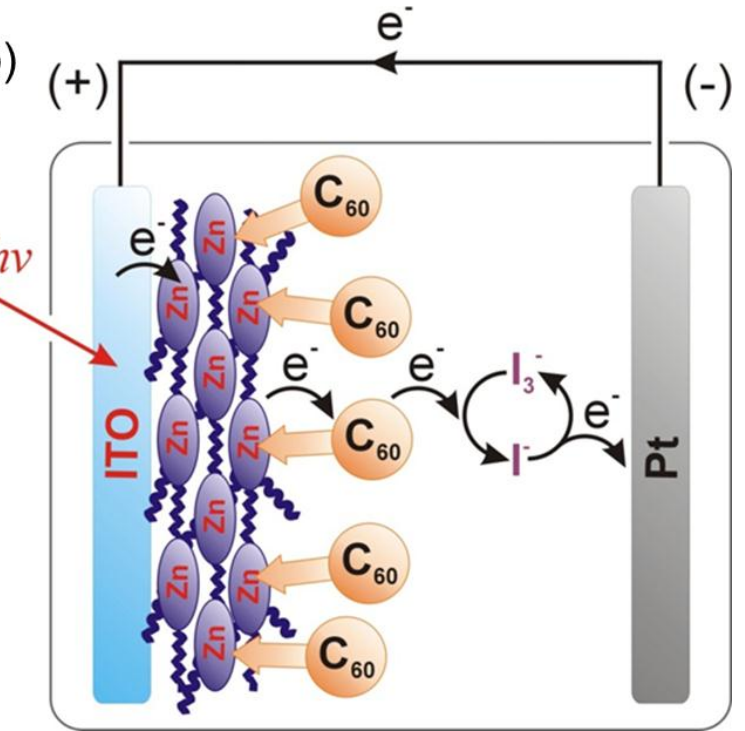

Figure 3.11: (a) Incident photon-to-current conversion efficiency (IPCE) for the $\left(\mathrm{Ph}_{2} \mathrm{~N}\right)_{4} \mathrm{ZnP}$ polymer film coated ITO electrode (10 cycles at $\left.25 \mathrm{mV} / \mathrm{s}\right)$ in (1) absence and (2) presence of the coordinated $\mathrm{C}_{60} \mathrm{im}$ ligand, in acetonitrile solution of the $\mathrm{I}_{3}{ }^{-} / \mathrm{I}^{-}(0.1$ $\mathrm{M} / 0.1 \mathrm{M}$ ) mediating redox couple. (b) Schematic view of the mechanism of the cathodic photocurrent generation by the $\left(\mathrm{Ph}_{2} \mathrm{~N}\right)_{4} \mathrm{ZnP}$ polymer film]-fullerene modified optically transparent ITO electrode

\subsection{Summary}

A tetrakis(4-(N,N-diphenylamino)phenyl)porphyrinatozinc(II) monomer bearing electroactive triphenylamine substituents was successfully electropolymerized to form thin films onto the $\mathrm{Pt}, \mathrm{Au}$, and ITO electrodes. This film formation was monitored with EQCM under both batch and FIA conditions and the $\left(\mathrm{Ph}_{2} \mathrm{~N}\right)_{4} \mathrm{ZnP}$ polymer film topography was imaged by using AFM in a tapping mode. These studies unraveled formation of a robust, dense and electrochemically active $\left(\mathrm{Ph}_{2} \mathrm{~N}\right)_{4} \mathrm{ZnP}$ polymer film. The zinc porphyrin $\pi$-electron system was preserved in the polymer, as confirmed by the presence of its characteristic optical absorption and emission bands. Further, fullerene, 
derivatized with an axially coordinating imidazole addend, was allowed to coordinate by self-assembly to the zinc atom center of the $\left(\mathrm{Ph}_{2} \mathrm{~N}\right)_{4} \mathrm{ZnP}$ moiety of the polymer film. The formation of the $\left[\left(\mathrm{Ph}_{2} \mathrm{~N}\right)_{4} \mathrm{ZnP}\right.$ polymer film]-fullerene hybrid was monitored by EQCM under FIA conditions. This hybrid was characterized by CV that allowed us to evaluate the formal redox potential of both the $\left(\mathrm{Ph}_{2} \mathrm{~N}\right)_{4} \mathrm{ZnP}$ donor and $\mathrm{C}_{60}$ acceptor moiety. The steady-state emission spectral data along with the free-energy calculations suggested occurrence of vectorial electron transfer from the singlet excited zinc porphyrin moiety of the polymer to the axially coordinated fullerene moiety. Moreover, the systematic photoelectrochemical studies unraveled (i) cathodic photocurrent generation in the $\left(\mathrm{Ph}_{2} \mathrm{~N}\right)_{4} \mathrm{ZnP}$ polymer film modified electrode, (ii) the opposite effect of thickness of the $\left(\mathrm{Ph}_{2} \mathrm{~N}\right)_{4} \mathrm{ZnP}$ polymer film on the generated photocurrent density and, finally, (iii) the photocurrent and photovoltage enhancing role of the coordinated fullerene ligand. The present study unambiguously revealed the importance of the fullerene in elevating the photochemical conversion efficiency of light into electricity. The IPCE value in the Soret region of the maximum absorption was nearly $2 \%$ for the present $\left[\left(\mathrm{Ph}_{2} \mathrm{~N}\right)_{4} \mathrm{ZnP}\right.$ polymer film]-fullerene hybrid film photoelectrochemical cell. 


\section{CHAPTER 4}

\section{SUPRAMOLECULAR DSSCs BASED ON AXIAL LIGATION}

4.1 Surface Modification of Nanocrystalline $\mathrm{TiO}_{2}$ with Coordinating Ligands to Immobilize Sensitizers and Dyads ${ }^{\dagger}$

\subsubsection{Introduction}

In nature, self-assembly through non-covalent binding motifs, ${ }^{150}$ such as hydrogen bonding, metal-ligand coordination, electrostatic, $\pi-\pi$ interactions and weak van der Waals interactions play dominant role. For example, photosynthetic antennareaction center pigments use such intermolecular forces to arrange precisely the donoracceptor entities in a protein matrix, exhibiting a cascade of vectorial energy and electron transfer processes. ${ }^{151}$ Inspired by this revelation, several groups have constructed supramolecular photosynthetic architectures to mimic the photoinduced energy and electron transfer processes, with an ultimate goal of building efficient light energy harvesting devices based on these bio-mimetic principles. ${ }^{152-159}$ The solar cells built based on the bio-mimetic principles could serve as an alternative to inorganic semiconductor based ones for renewable energy production. Here, the design and construction of electron- and hole-transporting, nanostructured architectures are pivotal for enhancing both charge separation efficiency and charge carrier mobility so as to improve the photovoltaic response. ${ }^{154}$ Although donor-acceptor type dyads have been frequently utilized for this purpose, ${ }^{14,152,153,155-157,159}$ examples of employing noncovalent methodology, especially the highly versatile metal-ligand binding

\footnotetext{
${ }^{\dagger}$ Section 4.1 in its entirety is reproduced from the Journal of American Chemical Society, 2009, 131(41), pp 14646- 14647 with permission from the publisher.
} 
approach $^{129,160,161}$ to assemble the different entities on the electrode surface have been scarce. $^{60}$

Here, we report a metal-ligand axial coordination approach ${ }^{129,160,161}$ to assemble zinc tetrapyrrole sensitizers as well as a (donor) $)_{1}$ (donor) $)_{2}$ type dyad instead of the frequently used donor-acceptor type dyads ${ }^{14,152,153,155-157,159}$ on semiconducting $\mathrm{TiO}_{2}$ surface. As demonstrated here, the present approach not only allows testing the photoelectrochemical behavior of different zinc tetrapyrroles but also allows us to utilize fairly complex structures involving more than one donor entity. Additionally, utilization of the dyad markedly improves the current-voltage (I-V) performance of the photoelectrochemical cell, due to an electron transfer-hole migration mechanism.<smiles>O=C(O)c1ccncc1</smiles>

1<smiles>O=C(O)c1ccncc1F</smiles>

2



3<smiles>O=C(O)c1ccc(-n2ccnc2)cc1</smiles>

$4 \prod_{N}$<smiles>C[13CH2]</smiles>

AND Enantiomer
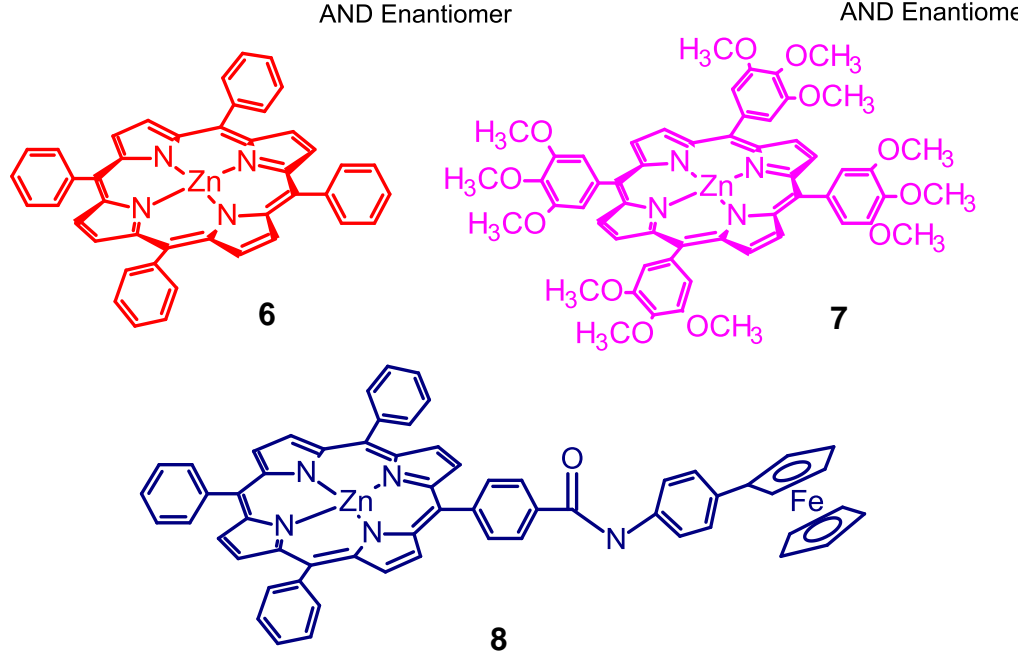<smiles>O=C(O)c1ccccc1</smiles>

AND Enantiomer

Scheme 4.1.1: Structures of the nitrogenous ligand possessing carboxylic acids used to modify the $\mathrm{TiO}_{2}$ surface (1-4); sensitizers (6-7) and the dyad (8) used to axially coordinate the immobilized nitrogenous bases on $\mathrm{TiO}_{2}$ surface. $\mathbf{5}$ is a control compound lacking the axial ligand entity. 


\subsubsection{Results and Discussion}

\subsubsection{Optical Studies}

In a typical experiment, a thin nanocrystalline $\mathrm{TiO}_{2}$ film coated $\mathrm{FTO}$ electrode ( 10-12 microns, tec7 from Pilkington) was surface modified with axial coordinating ligand bearing carboxylic acid (compounds 1-5 in scheme 4.1.1) by placing the electrode in an ethanolic solution of the desired compound overnight. After removal of the unbound molecules (2-3 ethanol washings), the $\mathrm{TiO}_{2}$ electrode was immersed in an o-dichlorobenzene (DCB) solution containing the desired zinc tetrapyrrole (6-8 in scheme 4.1.1) for 20-30 min. After this, the electrodes were rinsed with DCB to remove excess of uncoordinated zinc complex. Figure 4.1.2a shows the picture of the sensitizers in solution and upon surface modification. Corrected absorbance in the range of $0.14-0.37$ for the Soret band of the sensitizers was obtained (Figure 4.1.1a \& 4.1.1b).
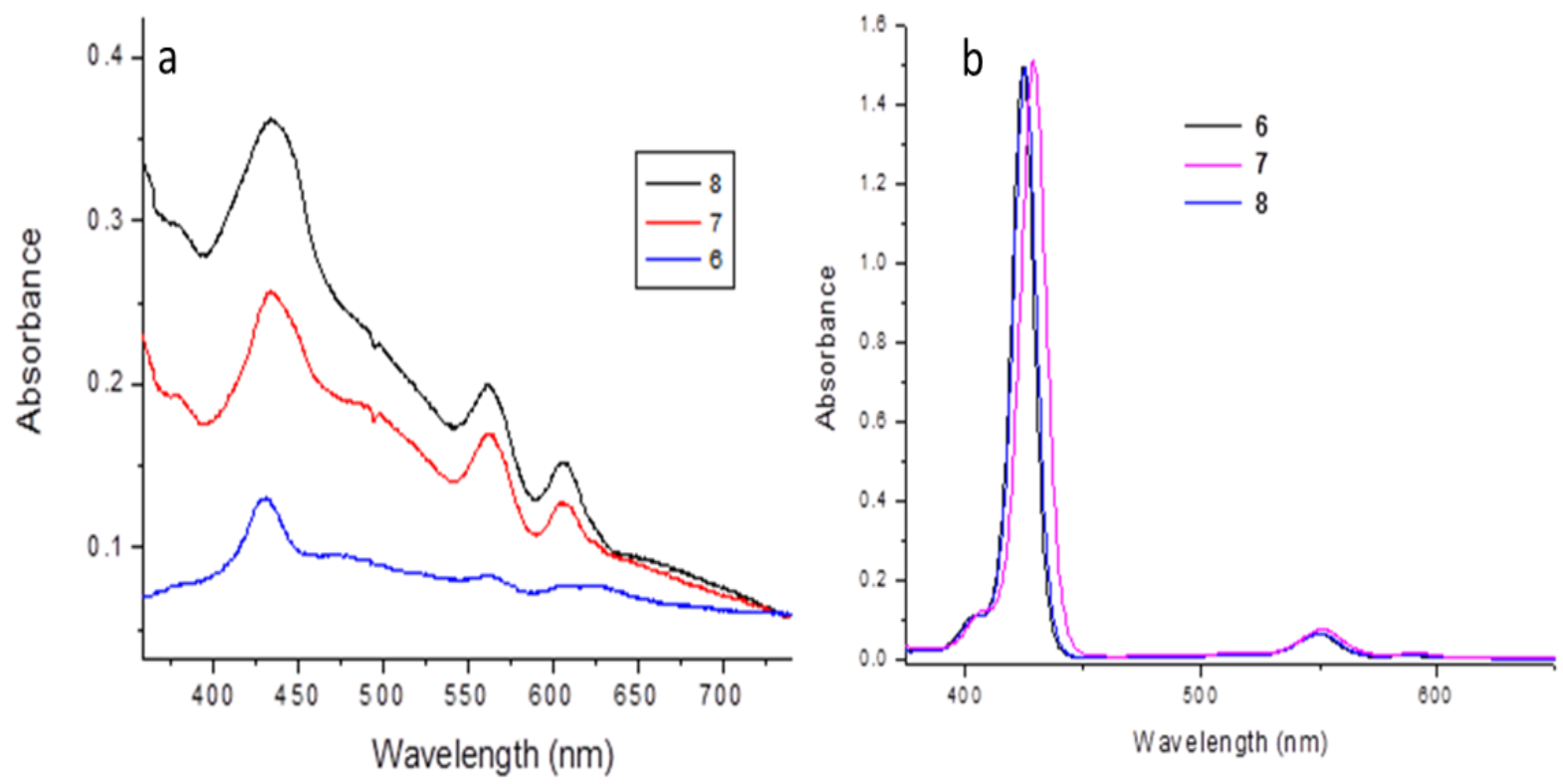

Figure 4.1.1: a) UV-visible spectrum of the sensitizer and dyad immobilized on 4 modified $\mathrm{FTO} / \mathrm{TiO}_{2}$ electrodes after correcting for $\mathrm{FTO} / \mathrm{TiO}_{2}$ absorbance. b) UV-Visible spectrum of the sensitizers and the dyad employed in the present study. 
When $\mathrm{TiO}_{2}$ was modified with $\mathbf{5}$ lacking axial coordinating ligand, some adsorption of the sensitizer occurred with optical density less than 0.05 (see Figure 4.2.1a). These results indicate effective immobilization via axial coordination of the zinc tetrapyrroles ${ }^{60,129,160-162}$ in the case of 1-4 modified $\mathrm{TiO}_{2}$ surface.

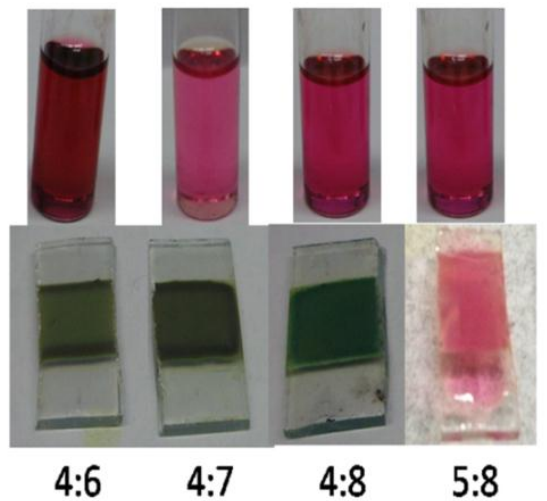

(a)

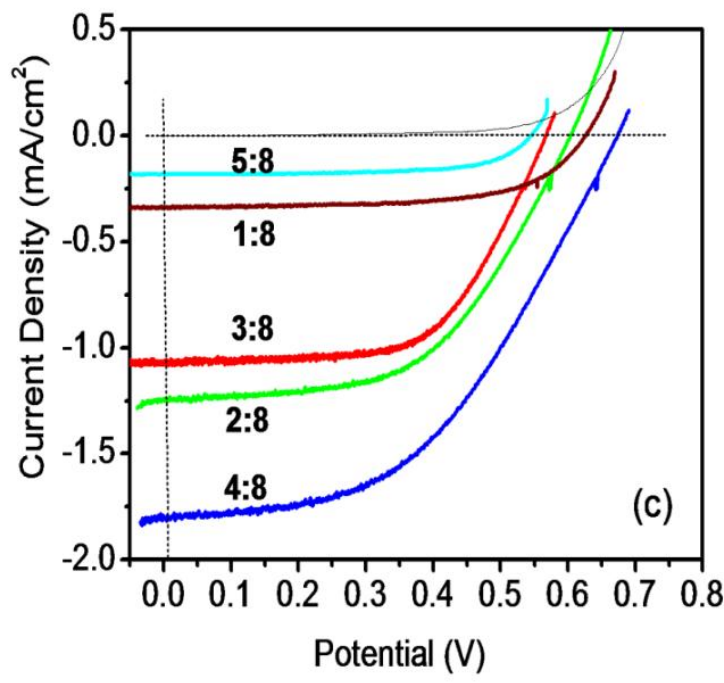

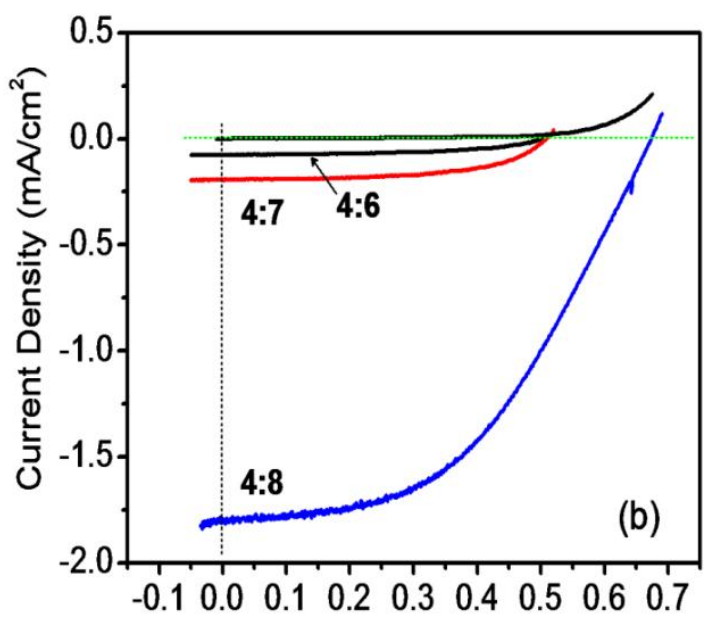

Potential (V)

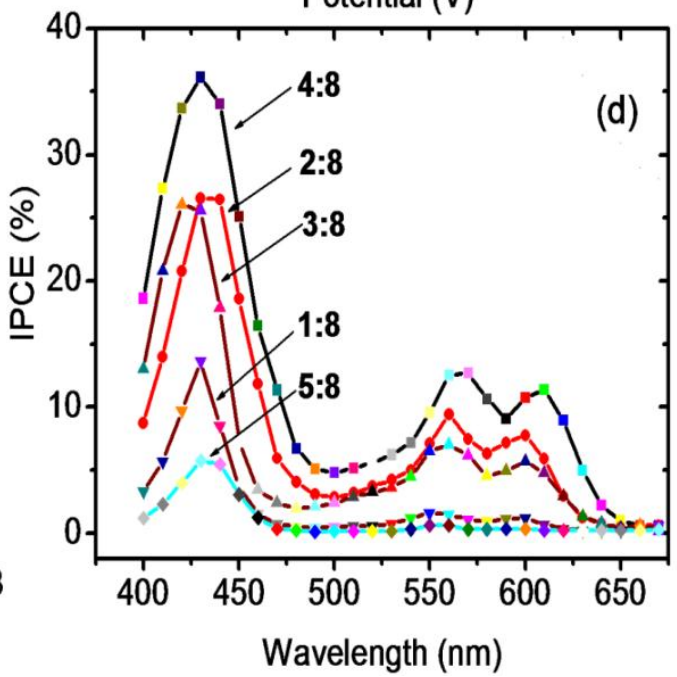

Figure 4.1.2: (a) Pictures of sensitizers in solution and on the $\mathrm{FTO}-\mathrm{TiO}_{2}$ modified with 4 or 5 electrodes, (b) $I-V$ characteristics showing the effect of different sensitizers bound to 4 modified $\mathrm{TiO}_{2}$, and (c) $I-V$ characteristics showing the effect of different surface modifiers on the photoelectrochemical behavior upon binding 8, and (d) IPCE curves of for the electrodes shown in (c). $\mathrm{I}-\mathrm{V}$ curves were generated in DCB containing $\mathrm{I}_{3}^{-} / \mathrm{I}^{-}(0.5$ $\mathrm{M} / 0.03 \mathrm{M}$ ) redox mediator using an AM 1.5 simulated light source with a $340 \mathrm{~nm}$ UVcut off filter. The black lines in $b$ and $c$ are dark currents recorded for the 4:8 modified electrodes 


\subsubsection{Photoelectrochemical Studies}

Photoelectrochemical cells were constructed using platinized FTO as counter electrode in non-coordinating DCB containing $0.5 \mathrm{M}\left(n-\mathrm{Bu}_{4}\right) \mathrm{NI}$ and $0.03 \mathrm{M} \mathrm{I}_{2}$ as redox mediator. We have chosen four nitrogenous bases of different pKa values ${ }^{128}$ to modify the $\mathrm{TiO}_{2}$ surface; two zinc tetrapyrroles, 6-7 having slightly different spectral and redox behavior to demonstrate the versatility of the present method; and a zinc porphyrinferrocene, 8, (donor) $)_{1}$-(donor) $)_{2}$ type dyad to exhibit improved photoelectrochemical behavior as a result of electron transfer-hole migration mechanism.

Figure 4.1.2b shows typical $I-V$ plots for sensitizers bound to 4 modified $\mathrm{TiO}_{2}$. Similar looking I-V plots, however with lower photocurrents, were obtained for electrodes modified with surface modifiers 1-3. The photocurrents, Isc generated for 4:6 and 4:7 modified electrodes were nearly an order of magnitude smaller compared to that obtained for the dyad 4:8 modified electrode. The photovoltage, $V_{O C}$ were also found to be smaller for the former electrodes. For the 4:8 modified electrode, the $V_{O C}$ and $I_{\mathrm{Sc}}$ were found to be $0.67 \mathrm{~V}$ and $1.6 \mathrm{~mA} / \mathrm{cm}^{2}$, respectively. Further, the performance of this dyad was tested for other surface modifiers. As shown in Figure 4.1.2c, higher currents were obtained for each of these electrodes compared to those modified with simple sensitizer. For a given zinc tetrapyrrole, the $I-V$ performance followed the order: $4>2>3>1>5$ surface modifier, that is, it largely followed the metalligand binding constants of a given zinc tetrapyrrole. ${ }^{128}$ The incident photon-to-current efficiency, IPCE plots for the electrodes described in Figure 4.1.2a are shown in Figure 4.1.2d. The shapes resembled that of absorbance spectra of the sensitizers (see Figures 4.1.1a for UV-Vis and 4.1.3a for IPCE of other sensitizer electrodes) suggesting 
that they are indeed responsible for the photovoltaic behavior. Additionally, photoswitching experiments revealed reproducible photocurrent generation (Figure 4.1.3b) indicating little or no dissociation of the coordinated sensitizer. The IPCE at the Soret band location were found to be $37 \%, 28 \%, 26 \%, 14 \%$ and $6 \%$, respectively for $4,2,3,1$ and 5 surface modified with the dyad, 8 electrodes. For the $4: 8$ modified photocell, the fill-factor and conversion efficiencies were found to be $47 \%$ and 0.56 respectively.
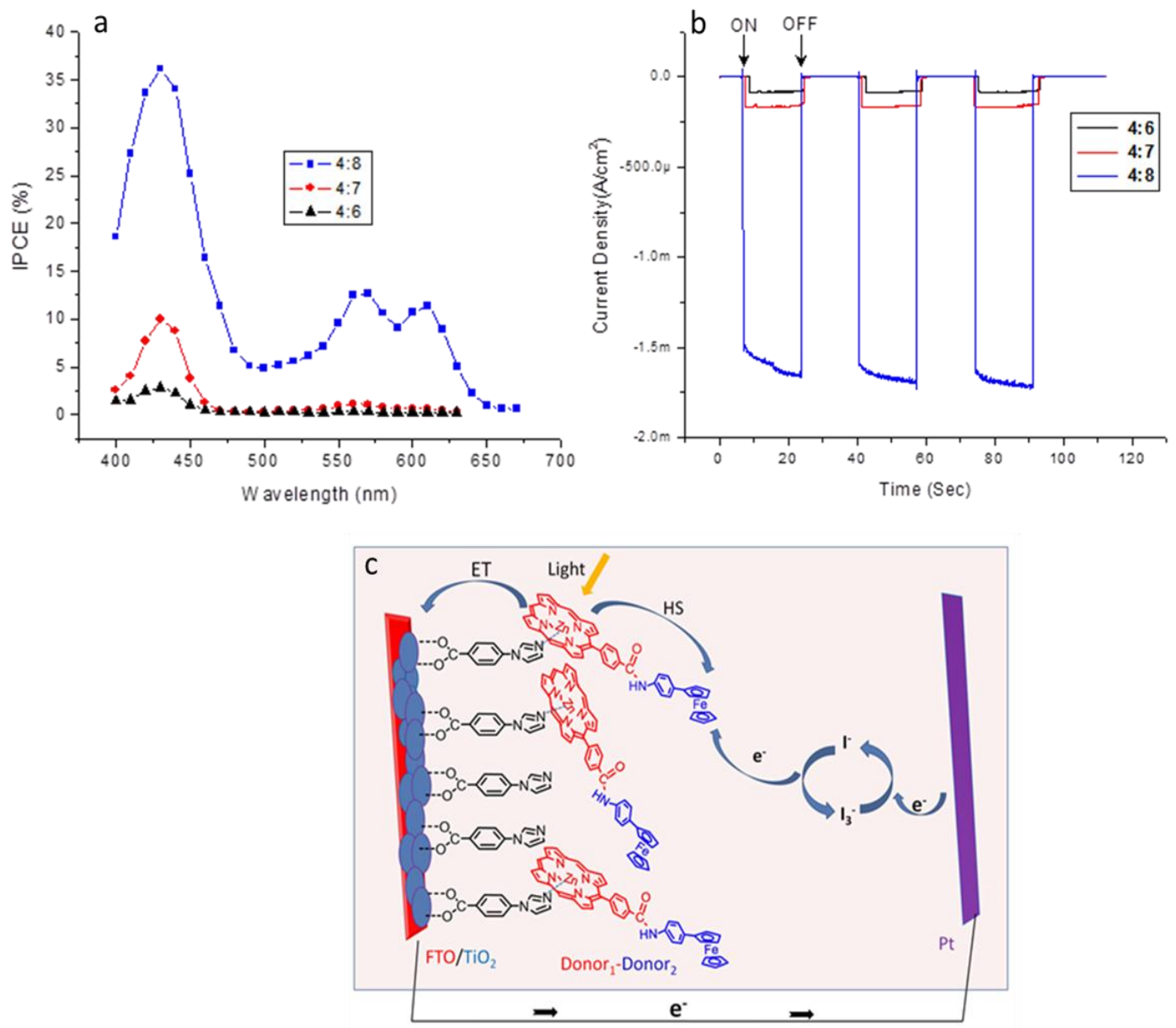

Figure 4.1.3: a) IPCE curves for 4:8, 4:7 and 4:6 modified $\mathrm{TiO}_{2} / \mathrm{FTO}$ in $\mathrm{DCB}$ containing $\mathrm{I}_{3}{ }^{-} / \mathrm{I}^{-}(0.5 \mathrm{M} / 0.03 \mathrm{M})$ redox mediator. b) Effect of light switching on photocurrent of the studied systems immobilized onto $\mathrm{TiO}_{2} /$ FTO surface. The switching experiments were performed in DCB containing a $0.5 \mathrm{M}(\mathrm{n}-\mathrm{Bu})_{4} \mathrm{NI}$ and $0.03 \mathrm{M} \mathrm{I}_{2}$ as redox mediator. c) Schematics of zinc porphyrin-ferrocene dyad (8) surface modified via axial coordination of 4 immobilized onto the $\mathrm{TiO}_{2}$ film on the FTO electrode. The photochemical and redox processes are shown by arrows. 
The photochemical events resulting in enhanced photocurrent in the case of ZnP-Fc (donor) $)_{1}$-(donor) $)_{2}$ dyad modified $\mathrm{TiO}_{2}$ electrode is shown in Figure 4.1.3c. Excitation of the donor zinc porphyrin of the dyad results in injecting an electron into $\mathrm{TiO}_{2}$ generating $\mathrm{ZnP}^{++}$. Owing to favorable redox potentials, ${ }^{18}$ a hole migration subsequently occurs to neutralize the $\mathrm{ZnP}^{++}$resulting into the formation of $\mathrm{Fc}^{+}$. The mediator, $\mathrm{I}^{-}$neutralizes the $\mathrm{Fc}^{+}$and forms $\mathrm{I}_{3}^{-}$which is subsequently reduced by the photo-ejected electron via the counter electrode thus closing the circuit. The present electron transfer-hole migration mechanism ${ }^{163}$ differs from the sequential electron transfer (photoexcited donor to the acceptor to the $\mathrm{TiO}_{2}$ ) often observed in the case of donor-acceptor modified electrodes ${ }^{14,152,153,155-157,159}$ signifying the importance of the presence biomimetic approach for superior photovoltaic performance.

\subsubsection{Summary}

We have devised an elegant method of self-assembly to modify $\mathrm{TiO}_{2}$ surface using coordinating ligands followed by immobilization of variety of sensitizers and a dyad. A maximum IPCE value of $37 \%$ was achieved for the $\mathrm{TiO}_{2}$ electrode modified with 4:8, a novel feature that was attributed to an electron transfer-hole migration mechanism of the dyad. Currently, utilization of this approach to modify nanocrystalline $\mathrm{TiO}_{2}$ and other semiconductor surfaces using different (donor) $)_{1}$-(donor) $)_{2}$, donor-acceptor and antenna-donor type dyads and triads, and evaluating their photoelectrochemical performance, kinetics of electron ejection and hole migration are in progress in our laboratory. 


\subsection{Effect of Metal Atom and Solvent Optimization}

\subsubsection{Introduction}

Zinc porphyrins and zinc phthalocyanines have been widely used as donors in the design and construction of donor-acceptor systems ${ }^{164,165}$. Several covalently and non-covalently linked zinc macrocyle - fullerene dyads have been synthesized and studied. Several zinc tetrapyrrole macrocycles have been used for the construction of photoelectrochemical cells using techniques such as: LB films, self-assembled monolayers, layer by layer ion pairing, spin coating hydrogen bonded systems and axial coordination ${ }^{13,14,62,130,156,164,166}$. Use of axially coordinated non carboxylic acid porphyrin compounds as sensitizer for DSSCs has been studied recently ${ }^{167}$. Using this approach, zinc tetraphenyl porphyrin (ZnTPP), substituted zinc porphyrins and fairly complex molecules have been used as sensitizer for DSSCs. Most of the axial coordination based DSSCs have employed only zinc metalated porphyrin macrocycle ${ }^{129,154,160,168}$. Axially coordinated zinc pthalocyanine based organic solar cells also showed improved performance than the uncoordinated systems ${ }^{130,169}$. Axially coordination based solar cells using ruthenium phthalocyanine ${ }^{170}$ showed poor performance due to lower recombination resistance.

To further understand the axial coordination interaction on metal oxide and also to improve the performance of the supramolecular system, varying the metal ion of the porphyrin cavity is another choice of interest. However only very few metalloporphyrins are fluorescent and could be found applicable for DSSCs application ${ }^{4,165,171,172}$. Among them, magnesium metalated porphyrin is known to be a fluorescent with diamagnetic metal ion at the centre. Magnesium atom is also present at the core of naturally 
occurring tetrapyrrole compounds cholorophylls ${ }^{3}$. Magnesium ion's abilty to axially coordinate helps to strengthen and improve the stability of the dimer in photosynthetic reaction center. Axial coordination of Maganesium ion containing porphyrin for DSSC applications have not been investigated, although, solution based studies have been reported ${ }^{173}$. Hence utilization of magnesium porphyrin without carboxylic acid as the dye has been explored here. Structures of zinc and magnesium tetraphenyl porphyrin compounds are shown in the Scheme 4.2.1. Various techniques were utilized to characterize the solar cells under similar conditions to understand photoelectrochemical events that are responsible for the generation of photocurrent.

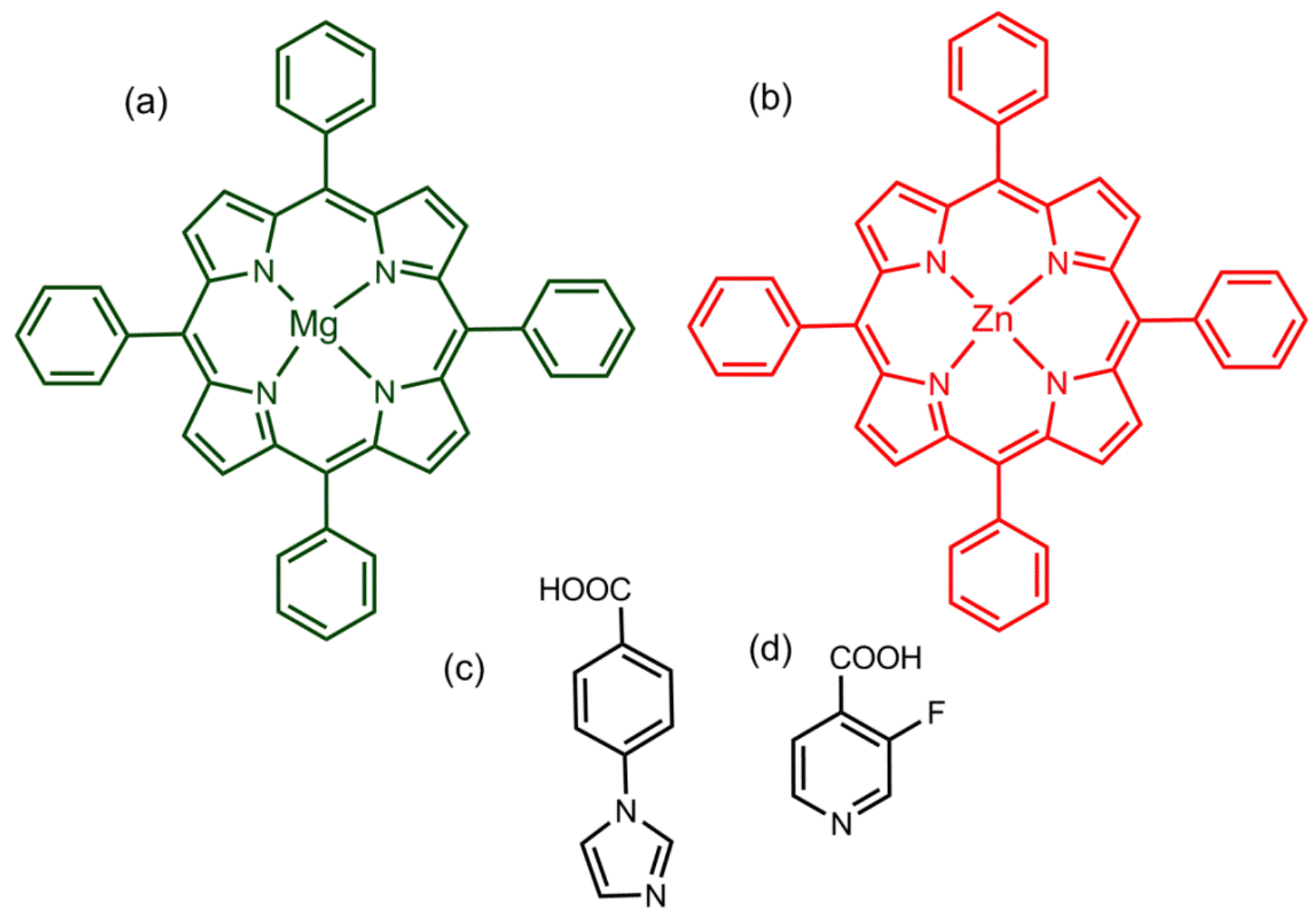

Scheme 4.2.1: Structure of a) Zinc tetraphenyl porphyrin (ZnTPP) and b) Magnesium tetraphenyl porphyrin (MgTPP) c) imidazole benzoic acid (im) d) 3-fluoropyridine 4carboxylic acid 


\subsubsection{Results and Discussion}

\subsubsection{Optical Studies}

Absorption spectrum of ZnTPP and MgTPP were very similar in odichlorobenzene (ODB) with an intense Soret band at $424 \mathrm{~nm}$ and $428 \mathrm{~nm}$ followed by two visible bands around $560 \mathrm{~nm}, 590 \mathrm{~nm}$ and $565 \mathrm{~nm}, 610 \mathrm{~nm}$ respectively as shown in Figure4.2.1a. It should be noted that the visible bands of MgTPP are 5-10 nm redshifted compared to ZnTPP. Both MgTPP $^{173}$ and ZnTPP ${ }^{174}$ upon axially coordinating with a ligand such as imidazole clearly showed isobestic points and red shift upon coordination in solution. To measure absorbance of coordinated metal complexes on a titanium dioxide surface, a thin layer of transparent $\mathrm{TiO}_{2}$ was formed on the FTO surface. $\mathrm{TiO}_{2}$ surface was modified with a carboxylic acid containing imidazole (c in scheme 4.2.1) coordinating ligand. Then the surface was immobilized with MgTPP and ZnTPP using axial coordination. Absorption spectra (Figure 4.2.1c) were recorded after cleaning the dye immobilized electrodes using Shimadzu Spectrometer. The absorption spectra were normalized for better comparison. As shown in the Figure there was clear red shift of $20 \mathrm{~nm}$ in the Soret bands in case of both porphyrins due to axial coordination. The visible bands of ZnTPP were also red shifted 5-10 nm, whereas MgTPP bands had very little effect. But the absorption intensity of MgTPP visible bands (500 nm - $600 \mathrm{~nm}$ region) was twice higher than ZnTPP. Thus both porphyrins were successfully immobilized and characterized using absorption spectroscopy. In order to understand the feasibility of electron injection into $\mathrm{TiO}_{2}$, excited state oxidation potential for both porphyrins were calculated using spectroscopic and electrochemical data listed on the Table 4.2.1. 

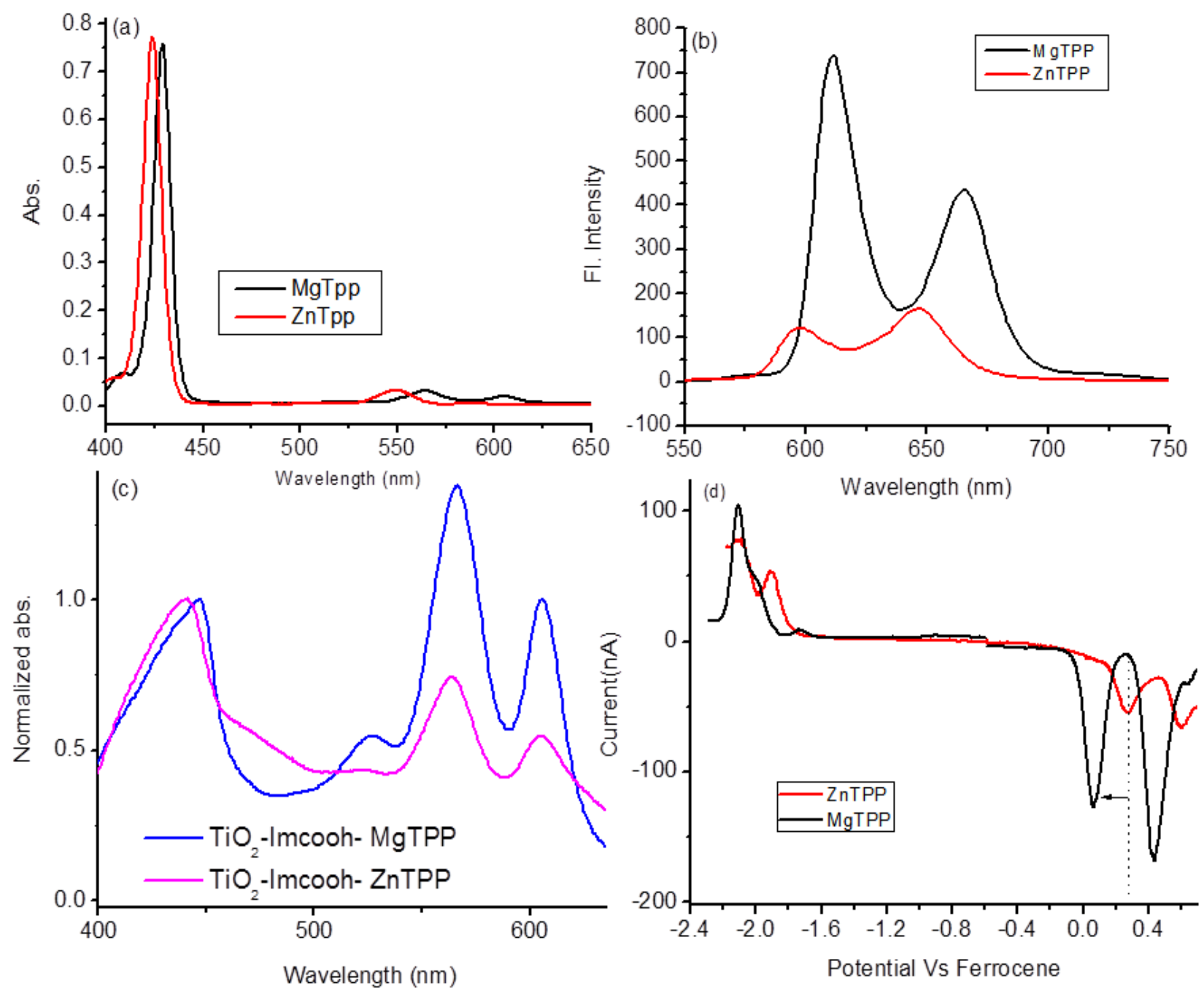

Figure 4.2.1: a) UV absorbance of the compounds measured in o-dichlorobenzene (DCB). b) Steady state emission spectrum of the compounds excited at their corresponding Soret band in ODB solvent. c) UV absorbance of the compounds after axially coordinating with the imidazole immobilized $\mathrm{TiO}_{2}$. d) Differential Pulse Voltammogram of the compounds measured in $0.1 \mathrm{M}$ Tetra butyl ammonium perchlorate in DCB.

From our previous studies, we found that the higher fluorescence quantum yield and longer life time of excited states for MgTPP compared to ZnTPP ${ }^{173}$. Cyclic Voltammogram of MgTPP and ZnTPP as shown in the Figure 4.2.1d, reveals the respective first oxidation potential to be at $0.07 \mathrm{~V}$ and $0.269 \mathrm{~V}$ vs ferrocene. So MgTPP is easier to oxidize than ZnTPP (200 mV). As a result, MgTPP would be expected to 
perform better compared to ZnTPP, due to lower driving force for charge recombination.

The free energy for charge recombination calculated as shown below ${ }^{175}$

$$
-\Delta G_{C R}=E_{O X}-E_{C B T i O 2}
$$

Under similar condition, the Gibbs' free energy change for recombination depends mainly on the oxidation potential of MgTPP and ZnTPP. Hence the driving force for charge recombination observed in case of ZnTPP will be higher compared to MgTPP. Also the calculated excited state oxidation potential of ZnTPP is about $300 \mathrm{mV}$ lower than that of MgTPP as shown in the Table 4.2.1. Both MgTPP (-1.403 V) and ZnTPP ($1.184 \mathrm{~V}$ ) have excited state oxidation potential higher than the $\mathrm{TiO}_{2}$ conduction band, but MgTPP could be expected to perform better than ZnTPP upon axial coordinating on $\mathrm{TiO}_{2}$ surface.

Table 4.2.1: Optical and electrochemical properties of MgTPP and ZnTPP. $E_{0-0}$ was calculated from the average of absorbance and emission peak. Excited state oxidation potential $E_{o x}^{*}$ was calculated using the electrochemical oxidation potential $\left(E_{o x}\right) . E_{o x}{ }^{*}=$ $E_{(0-0)}-E_{o x}$

\begin{tabular}{ccccccccc}
\hline & Soret & Extn & Q bands & Emi & lifetime & $\mathbf{E}_{0-0} / \mathbf{V}$ & Eox/V & $\mathbf{E}_{\text {ox }}^{*} / \mathbf{V}$ \\
\hline Dye & $\mathrm{nm}$ & $\times 10^{5} \mathrm{M}^{-1} \mathrm{~cm}^{-1}$ & $\mathrm{~nm}$ & $\mathrm{~nm}$ & $\mathrm{~ns}$ & {$[(\mathrm{abs} / \mathrm{Em}) / \mathrm{eV}]$} & Vs NHE & Vs NHE \\
ZnTPP & 424 & 5.74 & 588 & 597 & $2-2.5$ & 2.09 & 0.906 & -1.184 \\
& & & & & & & & \\
MgTPP & 428 & 5.38 & 605 & 611 & $5-10$ & 2.04 & 0.637 & -1.403 \\
\hline
\end{tabular}

\subsubsection{Computational Studies}

B3LYP/3-21G( $\left.{ }^{*}\right)$ calculations were performed to locate the position of HOMOLUMO orbital as well orientation of porphyrins with respect to $\mathrm{TiO}_{2}$. It is believed that bidentate mode is the most common mode of binding of carboxylic acid on $\mathrm{TiO}_{2}{ }^{4}$. If we assume a similar type of binding occurs when using coordinating ligand bearing carboxylic acid then we could predict the orientation of dye molecules on the surface 
using this calculation. Orientation of porphyrin macrocycles was shown to be very important for efficient electron injection. Calculated orientation angle of $46^{\circ}$ with respect to the plane of carboxylic acid was observed for ZnTPP and MgTPP upon axially coordinated on the $\mathrm{TiO}_{2}$ surface. Using the optimized structures, as shown in Figure 4.2.2, HOMO is located on the metal atom containing macrocyle whereas LUMO is on the carboxylic acid group. Thus computational calculations address the possibility of existence of good electronic communication between metal oxide and due to axial coordination.
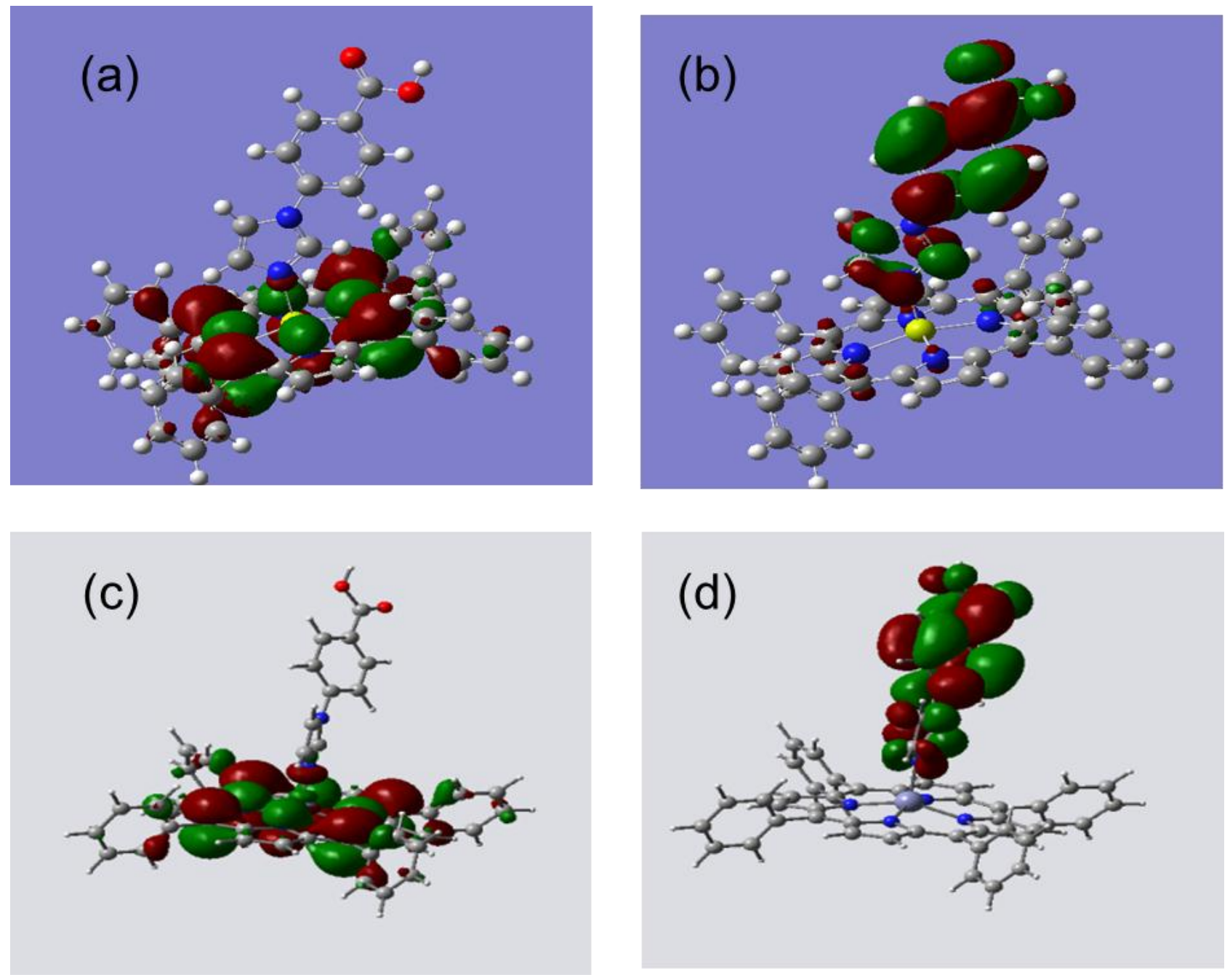

Figure 4.2.2: (a)-(b) HOMO-LUMO of ZnTPP and (c)-(d) HOMO- LUMO of MgTPP axially coordinating with imidazole benzoic acid. B3LYP/3-21G( $\left.{ }^{*}\right)$ optimized structures. 


\subsubsection{Photoelectrochemistry}

Supramolecular solar cells were constructed using the procedure for typical dye sensitized solar cells, with the modifications as described in previous chapter. Metal containing porphyrin compounds of similar concentrations in o-dichlorobenzene were used for dye immobilization with a soaking time of 30 minutes. After modification and careful washing, typical 2 electrode assembly were constructed using thermally deposited platinum on FTO as counter electrode. Then the electrolyte composed of 0.5 $\mathrm{M}$ TBAI and $0.05 \mathrm{M} \mathrm{I}_{2}$ in o-dichlorobenzene, was added in between the 2 electrodes using capillary forces.

As shown in the Figure 4.2.3a, current switching experiment showed short circuit current density $\left(I_{s c}\right)$ for MgTPP and ZnTPP of $1.5 \mathrm{~mA} / \mathrm{cm}^{2}$ and $0.19 \mathrm{~mA} / \mathrm{cm}^{2}$, respectively. Reproducible current indicates that dye molecules though axially coordinated stayed robust during photoelectrochemical measurement. In addition, the comparison of the results of MgTPP immobilized on different coordinating ligand modified $\mathrm{TiO}_{2}$ revealed phenyl imidazole as the best coordinating ligand, similar to our earlier report ${ }^{167}$ (see Figure 4.2.3b). Also absence of coordinating ligand on $\mathrm{TiO}_{2}$ surface resulted in poor physical adsorption and hence the performance as shown in Figure 4.2.3b. $\mathrm{J}-\mathrm{V}$ plot in Figure 4.2.3c, under similar conditions revealed MgTPP exhibits about $100 \mathrm{mV}$ higher open circuit potential $\left(V_{o c}\right)$ than ZnTPP in addition to higher short circuit current density. The fill factor and efficiency of MgTPP based cell were calculated to be $49 \%$ and $0.40 \%$ respectively. Incident photon to current conversion efficiency (IPCE) calculations also showed that MgTPP has better conversion efficiency compared to ZnTPP over the Soret band ( $28 \%$ to $3.8 \%$ ) as well as visible bands $(15 \%$ to $<1 \%$ ) as 
shown in Figure 4.2.3d. It is worth mentioning that the IPCE and absorption spectra in the Figure 4.2.1 matched well.
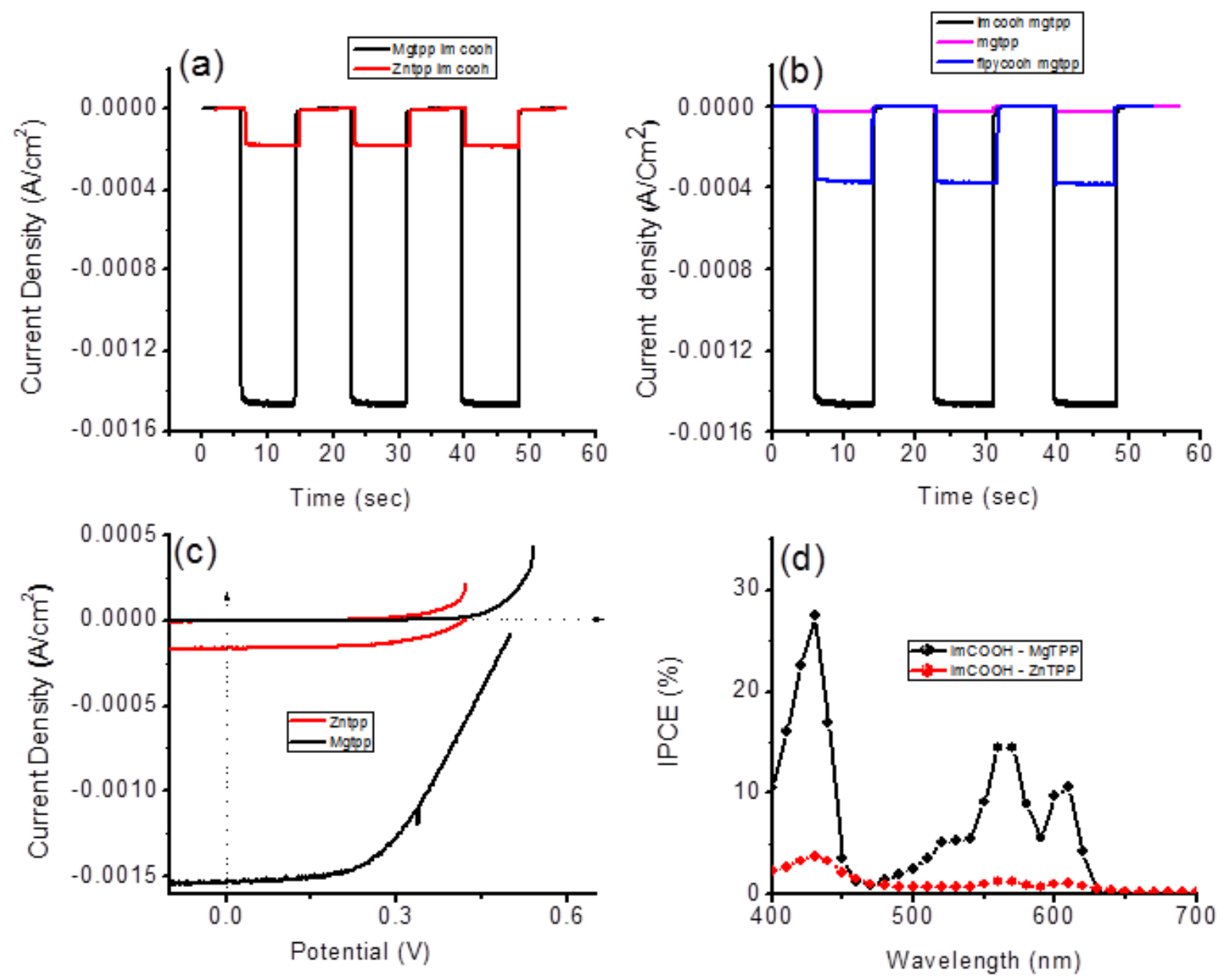

Figure 4.2.3: (a) Photocurrent switching of MgTPP and ZnTPP immobilized on $\mathrm{TiO}_{2}$. (b) Photocurrent switching of MgTPP adsorbed different coordinating ligands on $\mathrm{TiO}_{2}$. (c) $\mathrm{J}$ $V$ characeristics of MgTPP and ZnTPP immobilized on $\mathrm{TiO}_{2}$. (d) IPCE plot of MgTPP and $\mathrm{ZnTPP}$ immobilized on $\mathrm{TiO}_{2}$. All of the above experiments were performed in 0.5 TBAI $/ 0.05 \mathrm{M} \mathrm{I}_{2}$ in ODB.

The bigger difference in performance can be attributed to the strength of axial coordination between the metal centre and coordinating ligand, light absorption, and electrochemical properties of the immobilized dye. To understand the better performance in IPCE for MgTPP, Light Harvesting Efficiency ${ }^{176}$ for axially coordinated $\mathrm{ZnTPP} / \mathrm{TiO}_{2}-\mathrm{Im}$ and $\mathrm{MgTPP} / \mathrm{TiO}_{2}-\mathrm{Im}$ were calculated and shown in the Figure 4.2.4. 
LHE for MgTPP $(60 \%)$ at the Soret and $Q$ bands was almost twice as high when compared to that of ZnTPP (30\%). Thus, by changing the metal atom at the centre of tetrapyrrole macrocycle, significant improvement in light harvesting nature supramolecular solar cell was observed.

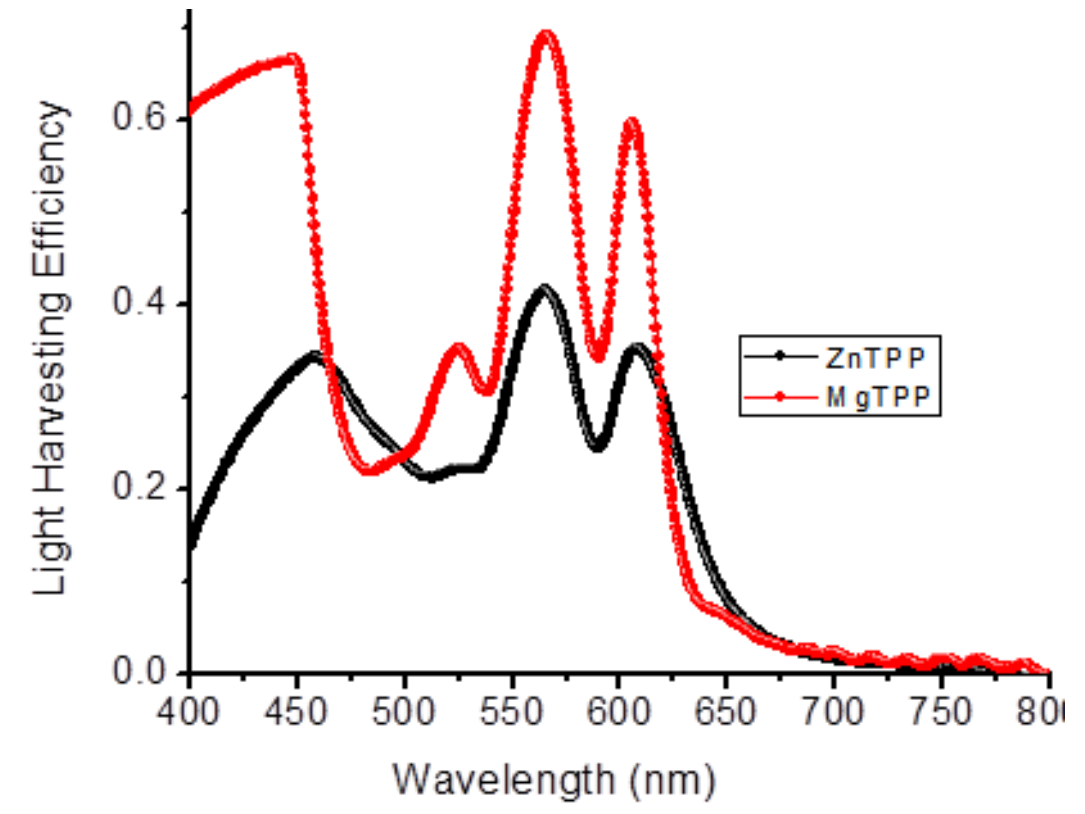

Figure 4.2.4: Light Harvesting Efficiency ${ }^{176}$ of the $\mathrm{MgTPP} / \mathrm{im}-\mathrm{TiO}_{2}$ and $\mathrm{ZnTPP} / \mathrm{im}-\mathrm{TiO}_{2}$ electrode.

\subsubsection{Fill Factor Optimization}

With preference of non-coordinating and high boiling point solvent for redox mediator solution, ODB still was the best choice. Since Lil or ionic liquids such as PMII were not soluble in ODB, we chose TBAI as our lodide source in the mediator. Compared to acetonitrile, the dielectric constant of ODB is lower. With the same concentration of iodide-iodine, using mixture of solvents the overall dielectric constant of the electrolyte could be varied. So, redox electrolytes were prepared using acetonitrile and ODB mixture at different ratios as shown in the Figure 4.2.5. Using identically prepared $\mathrm{MgTPP} / \mathrm{TiO}_{2}$ electrodes, the effect of addition of acetonitrile on the 
performance of supramolecular DSSC was recorded and shown below. As the volume of acetonitrile was increased, two significant changes were observed as shown in the Figure 4.2.5a and 4.2.5b. First, the fill factor for the cell increased effectively from $49 \%$ to $72 \%$ with little addition of acetonitrile to ODB and then decreased to $66 \%$ when acetonitrile alone was used.

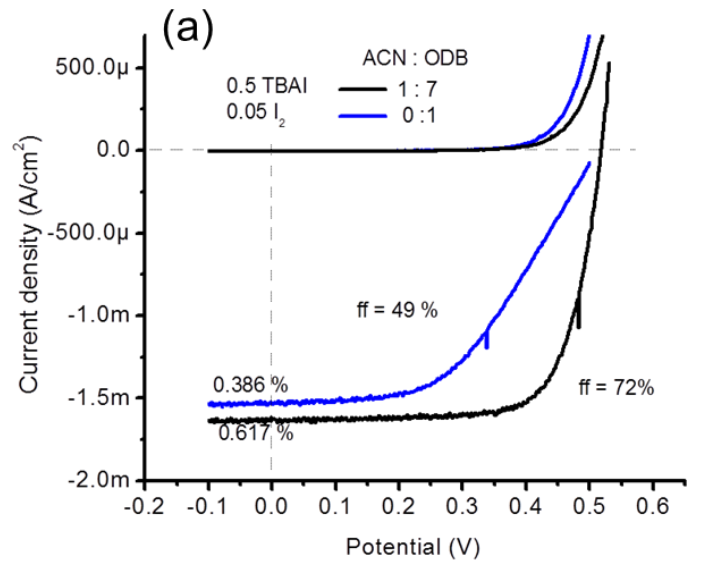

(c)

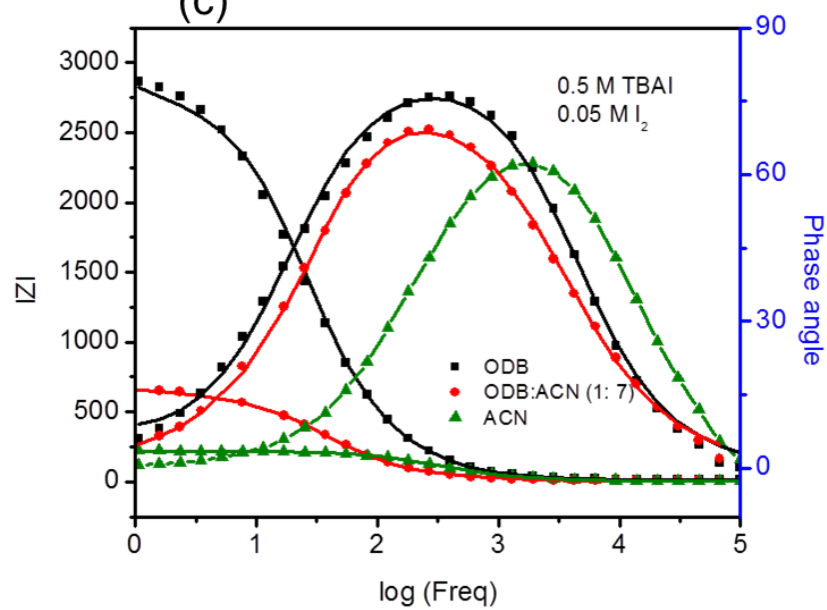

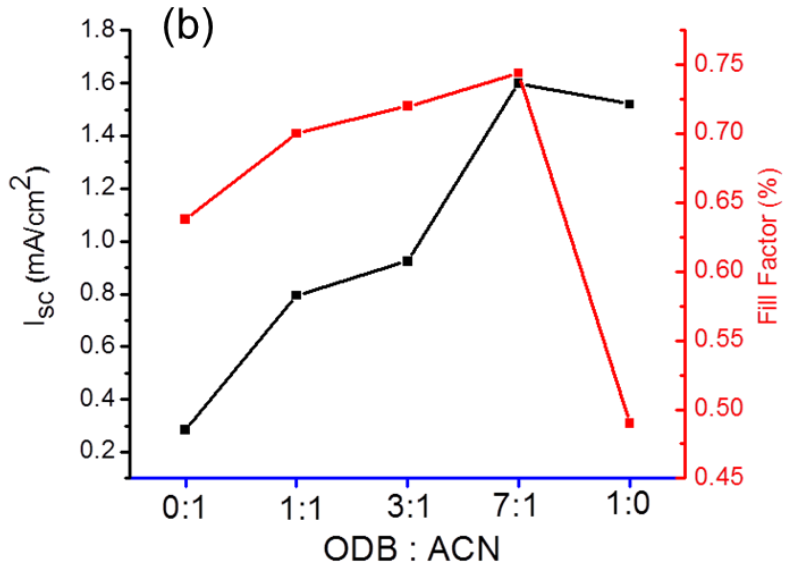

(d)

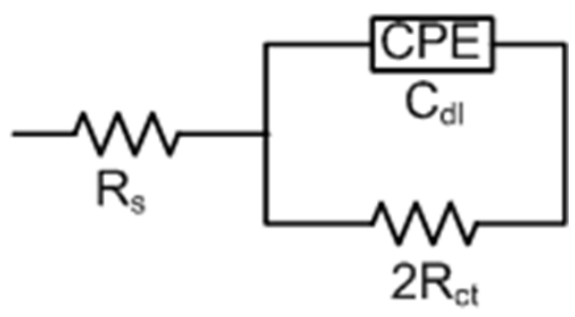

Figure 4.2.5: (a) $J-V$ plot of $\mathrm{MgTPP} / \mathrm{im}-\mathrm{TiO}_{2}$ in different electrolyte solvent conditions (b) Effect of electrolyte solvent conditions on the current density and fill factor of MgTPP/im$\mathrm{TiO}_{2}$ electrode. (c) Bode plot of impedance and phase angle vs log frequency of the two platinized FTO under symmetric electrode condition using different electrolyte compositions. (d) Equivalent circuit used for fitting the impedance data $R s$ - solution resistance, $R_{c t}$ - charge transfer resistance for at Pt/electrolyte interface and $C_{d l}$ is the double capacitance of the same interface. All of the above experiments were performed in mentioned solvents with $0.5 \mathrm{TBAl} / 0.05 \mathrm{M} \mathrm{I}_{2}$ as redox mediator. 
At the same time, short circuit current density $\left(J_{s c}\right)$ was around $1.6 \mathrm{~mA} / \mathrm{cm}^{2}$ initially and then decreased drastically to $0.2 \mathrm{~mA} / \mathrm{cm}^{2}$ with increase in the amount of acetonitrile in the electrolyte. Overall, performance of MgTPP sensitized improved with energy efficiency of $0.63 \%$ and corresponding fill factor of $72 \%, 1.6 \mathrm{~mA} / \mathrm{cm}^{2} J_{s c}$ and $0.519 \mathrm{~V}$ upon using mixed solvent based redox mediator. Low short circuit current density was expected in the case of acetonitrile alone conditions, as it is more polar compared to ODB and competes in axial coordination, resulting in desorption of the dye. The improvement in the performance (Figure 4.2.5a) due to smaller addition acetonitrile to ODB (1:7) is being attributed to significant decrease in the resistance at platinum electrolyte interface facilitating better iodide/iodine mediator and dye regeneration ${ }^{177}$. In order to prove, EIS spectra were recorded using corresponding electrolyte systems with a symmetric platinum electrode configuration ${ }^{178}$. As shown in the Nyquist plot (Figure 4.2.5c) with small addition of acetonitrile the charge transfer resistance $\left(R_{c t}\right)$ at the platinum electrolyte interface decreased by almost $50 \%$ from $1305 \Omega \mathrm{cm}^{2}$ to $775 \Omega \mathrm{cm}^{2}$ with the very small addition of acetonitrile.

Table 4.2.2: Solvent resistance $\left(R_{s}\right)$, charge transfer resistance $\left(R_{c t}\right)$ and double layer capacitance $\left(C_{d l}\right)$ of the symmetric $\mathrm{Pt}$ electrode configuration of different solvent containing $0.5 \mathrm{M}$ tetra butyl ammonium iodide and $0.05 \mathrm{M} \mathrm{I}_{2}$.

\begin{tabular}{cccc}
\hline Solvent & $R_{s}\left(\Omega \mathrm{cm}^{2}\right)$ & $2 R_{c t}\left(\Omega \mathrm{cm}^{2}\right)$ & $C_{d l}$ \\
\hline ODB (1) & 15.08 & 2610 & 3.6 \\
ACN:ODB(1:7) & 7.016 & 1558 & 3.6 \\
ACN (1) & 6.051 & 213 & 3.35 \\
\hline
\end{tabular}


The solution resistance also decreased from 15 to $7 \Omega \mathrm{cm}^{2}$. The least resistance was found to be for acetonitrile $213 \Omega \mathrm{cm}^{2}$ as shown in the Table 4.2.2. Because of the bigger cation in tetrabutyl ammonium iodide compared to Lil, the charge transfer resistance was very high. Bigger size cation and solvent conditions impeded us to further study the effect of metal centre on the recombination resistance of the corresponding metal oxide interface.

\subsubsection{Summary}

Supramolecular approaches to build dye sensitized solar cells enables alternative biomimetic route to be explored for improving the overall performance. Using axial coordination, non-carboxylic acid dye molecules could be easily self-assembled. Compared to zinc porphyrin as supramolecular dye, replacement of the metal ion to $\mathrm{Mg}$ atom showed significantly improved performance. Lower driving force for charge recombination followed by better light harvesting efficiency of MgTPP led to the overall improvement. The charge transfer resistance at the Pt/electrolyte interface decreased by two folds upon using mixed solvent. This in turn improved the overall fill factor of the system from $49 \%$ to $71 \%$ with an overall energy efficiency of $0.6 \%$. 


\section{CHAPTER 5}

\section{ANION BINDING BASED SUPRAMOLECULAR SYSTEMS}

5.1 Effect of Anion Binding on Charge Stabilization in a Bis-FullereneOxoporphyrinogen Conjugate ${ }^{\ddagger}$

\subsubsection{Introduction}

Control of electron-transfer processes is an important issue in molecular species that may lead to their application in synthetic organic light-harvesting systems or molecular electronic devices ${ }^{15,47,92,152,156,166,179-182}$. Control of electron-transfer processes is often made possible by adding external ionic cofactors, which favor either charge separation or recombination. ${ }^{183}$ For example, in the $\mathrm{O}_{2}$ evolving complex of photosystem II (PSII), $\mathrm{Ca}^{2+}$ and $\mathrm{Cl}^{-}$are known to be essential cofactors for efficient water oxidation. ${ }^{184,185}$ In this context, our labs previously reported a supramolecular oligochromophoric model system containing sites for binding of a reagent species and an anionic species $\left(\mathrm{F}^{-}\right)$in order to probe the effect of the binding of $\mathrm{F}^{-}$on the identities of products from photoinduced electron-transfer processes that occur within the resulting complex. ${ }^{186}$ However, the effects of anionic species on photoinduced electrontransfer processes via the singlet excited state in covalently linked electron donoracceptor systems, which are more chemically robust, have yet to be clarified.

We report herein the effect of binding of $\mathrm{F}^{-}$to an appropriately structured tetrapyrrole chromophore, which is covalently linked with electron acceptor units, on the photoinduced electron-transfer (PET) processes via both the singlet and triplet excited states. The chemical structure of the subject molecule $\operatorname{OXP}\left(\mathrm{C}_{60}\right)_{2}$ contains two

\footnotetext{
${ }^{\ddagger}$ Section 5.1 its entirety is reproduced from the Chemical Communication, 2010, 46, pp 7933- 7935 with permission from the publisher.
} 
fulleropyrrolidine $\left(\mathrm{C}_{60}\right)$ units substituted through $4,4^{\prime}$-biphenylmethylene groups at the nitrogen atoms of an oxoporphyrinogen (OXP) unit as depicted in Scheme 5.1.1. Both OXP and $\mathrm{C}_{60}$ of the triad are usually considered electron-accepting agents ${ }^{92,179,181,186}$ except that in this case the OXP unit is expected to be an electron donor given its ease of oxidation relative to the fulleropyrrolidine groups. Both OXP and $\mathrm{C}_{60}$ components are fluorescent with the doubly substituted OXP being more strongly emissive. ${ }^{92,179,181,187}$ Excitation of either of the entities is expected to induce photochemical processes in the triad. Significantly, the two imino hydrogens of the OXP unit possess the ability to bind anions with high stability ${ }^{188}$ resulting in modulation of the OXP unit redox properties. ${ }^{189}$ That is, the one-electron oxidation potential of the OXP unit, with four hemiquinone entities, is expected to exhibit large cathodic shifts upon binding of an anion. Consequently one should expect substantial changes in the energy levels which should alter the overall photochemical quenching processes.

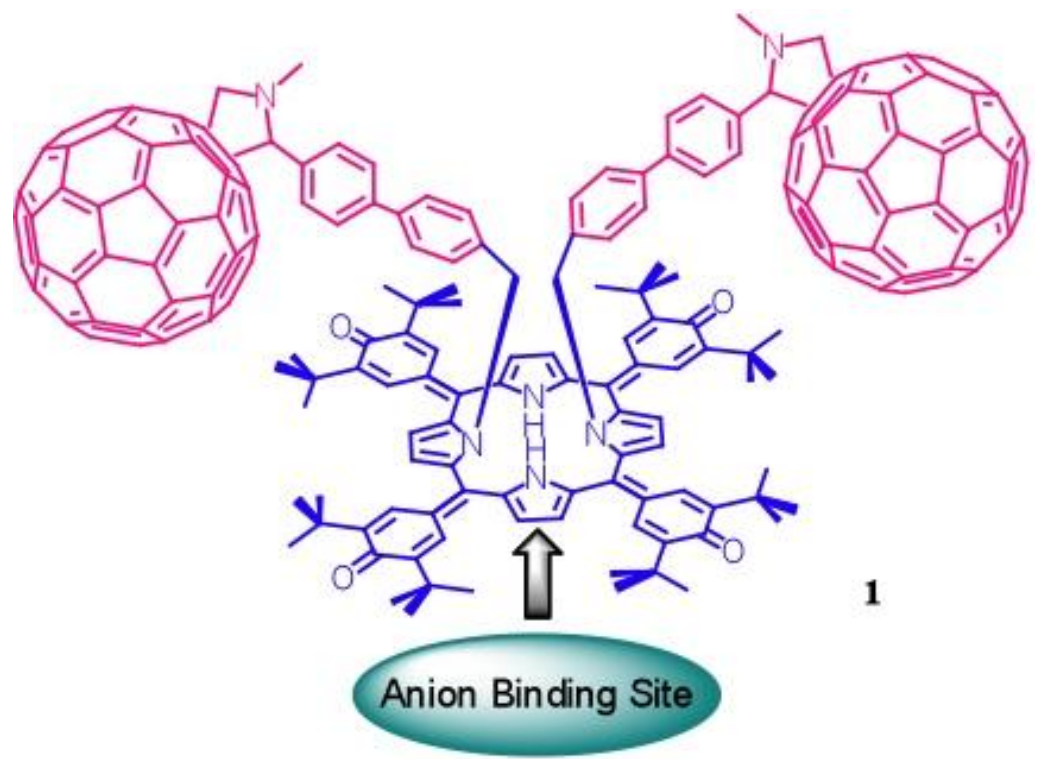

Scheme 5.1.1: Structure of the newly synthesized $\operatorname{OXP}\left(\mathrm{C}_{60}\right)_{2}$ triad, to probe anion binding effect on charge separation. 
This phenomenon has been tested in the present study by using the newly synthesized model compound $\mathrm{OXP}\left(\mathrm{C}_{60}\right)_{2}$.

\subsubsection{Experiments}

The following synthesis was done by students in Prof. Jonathan Hill's lab.

5.1.2.1 Synthesis of $N_{21}, N_{23}$-Bis[4-(4-formylphenyl)benzyl]-5,10,15,20-tetrakis(3,5-di-tbutyl-4-oxocyclohexa-2,5-dienyl)porphyrinogen

Synthesis of $\quad N_{21}, N_{23}$-Bis(4-bromobenzyl)-5,10,15,20-tetrakis(3,5-di-t-butyl-4oxocyclohexa-2,5-dienyl)- porphyrinogen $\left[(4 \mathrm{BrBz})_{2} \mathrm{OXP}\right]$ was prepared by a literature method. [1,1'-Bis(diphenylphosphino)ferrocene]dichloropalladium(II) dichloromethane complex (15 mg, 10 mol\%) was added to a degassed mixture of $(4 \mathrm{BrBz})_{2} \mathrm{OXP}(137$ $\left.\mathrm{mg}, 9.35 \times 10^{-5} \mathrm{~mol}\right)$, 4-formylphenylboronic acid (45 mg, $\left.3 \times 10^{-4} \mathrm{~mol}\right)$ and $\mathrm{K}_{2} \mathrm{CO}_{3}(0.1$ $\mathrm{g}$ dissolved in $\left.\mathrm{H}_{2} \mathrm{O}(1 \mathrm{~mL})\right)$ in a mixture of toluene $(10 \mathrm{~mL})$ and ethanol $(2 \mathrm{~mL})$. The resulting mixture was stirred at $60^{\circ} \mathrm{C}$ for $48 \mathrm{~h}$ under an atmosphere of dry nitrogen. The mixture was allowed to cool to room temperature and poured into water. The mixture was extracted with dichloromethane $(2 \times 40 \mathrm{~mL})$ and the combined organic fractions were dried over anhydrous $\mathrm{Na}_{2} \mathrm{SO}_{4}$. Solvents were subsequently evaporated under reduced pressure and the residue chromatographed on silica gel eluting with dichloromethane. Product containing fractions were combined and the solvents removed under reduced pressure yielding the desired product as a dark green solid, which was used without further purification. Yield: $104 \mathrm{mg}, 74 \%$. UV/Vis $\left(\mathrm{CH}_{2} \mathrm{Cl}_{2}\right): \lambda_{\max }$ $=508.5 \mathrm{~nm} .{ }^{1} \mathrm{H}-\mathrm{NMR}\left(\mathrm{CDCl}_{3}, 25^{\circ} \mathrm{C}, 300 \mathrm{MHz}\right): \delta=1.18\left(\mathrm{~s}, 36 \mathrm{H},{ }^{\mathrm{t}} \mathrm{Bu}-\mathrm{H}\right), 1.36(\mathrm{~s}, 36 \mathrm{H}$, $\left.{ }^{\mathrm{t}} \mathrm{Bu}-\mathrm{H}\right), 4.59$ (s, 4H, benzyl $\left.-\mathrm{CH}_{2}-\right), 6.58\left(\mathrm{~s}, 4 \mathrm{H}, \beta\right.$-pyrrole-H), $6.92\left(\mathrm{~d},{ }^{3} \mathrm{~J}=8.06 \mathrm{~Hz}, 4 \mathrm{H}\right.$, biphenylyl-H), 6.97 (s, 4H, cyclohexa-2,5-dienyl-H), 7.06 (s, 4H, $\beta$-pyrrole-H), 7.41 (d, ${ }^{3} \mathrm{~J}$ 
$=8.25 \mathrm{~Hz}$, biphenylyl- $\mathrm{H}$ ), $7.56\left(\mathrm{~d},{ }^{3} \mathrm{~J}=8.07 \mathrm{~Hz}, 4 \mathrm{H}\right.$, biphenylyl- $\left.\mathrm{H}\right), 7.63$ (s, 4H, cyclohexa-2,5-dienyl-H), 7.90 (d, ${ }^{3} \mathrm{~J}=8.07 \mathrm{~Hz}, 4 \mathrm{H}$, biphenylyl-H), 9.87 (br. s. $2 \mathrm{H}$, pyrrole-NH), 10.02 (s, 2H, formyl-H) ppm. ${ }^{13} \mathrm{C}-\mathrm{NMR}\left[\mathrm{CDCl}_{3}: d_{6}\right.$-DMSO (50:50 v/v), 25 $\left.{ }^{\circ} \mathrm{C}, 300 \mathrm{MHz}\right]: \delta=29.31,29.41,35.10,35.41,120.59,121.51,127.26,127.78,130.07$, 131.26, 131.55, 132.37, 135.31, 135.99, 137.69, 139.02, 145.65, 146.15, 147.12, 185.55 (formyl-C) ppm. FT-IR: v = 2997.8 (w, C-H str.), 2955.2 (m, C-H str.), 2919.6 (m), C-H str.), 2864.1 (m, C-H str.), 1702.9 (m, C=O str.), 1594.8 (s, C=C str.), 1563.0 (m), $1550.9(\mathrm{~m}), 1530.0$ (m), 1492.4 (s, 'Bu, asym. def.), 1453.3 (s, 'Bu, sym. bend.), 1405.7 (w), 1387.9 (w), 1360.4 (s, 'Bu, sym. bend.), 1334.2 (w), $1317.6(\mathrm{~m}), 1264.0(\mathrm{~m})$, $1228.5(w), 1202.7(w), 1169.4(w), 1088.4(m), 1040.2(w), 1028.4(m), 1005.4(w)$, $949.0(m), 929.6,(w), 886.6(w), 840.5(w), 818.7(w), 801.2(m), 757.7(m), 743.7(m)$ $\mathrm{cm}^{-1}$. MALDI-TOF-MS (dithranol): $[\mathrm{M}+2 \mathrm{H}]^{+}\left(\mathrm{C}_{104} \mathrm{H}_{114} \mathrm{~N}_{4} \mathrm{O}_{6}\right)$ : calcd. 1516.88; found 1516.99.

\subsubsection{Synthesis of $\operatorname{OXP}\left(\mathrm{C}_{60}\right)_{2}$ Triad}

$N_{21}, N_{23}$-Bis[4-(4-formylphenyl)benzyl]-5,10,15,20-tetrakis $\quad$ (3,5-di-t- $\quad$ butyl-4oxocyclohexa-2,5-dienyl) porphyrinogen $\left(51 \mathrm{mg}, 3.37 \times 10^{-5} \mathrm{~mol}\right) \mathrm{C}_{60}(50 \mathrm{mg}, 6.94 \times$ $10^{-5} \mathrm{~mol}$ ) and $\mathrm{N}$-methylglycine $\left(62 \mathrm{mg}, 6.97 \times 10^{-4} \mathrm{~mol}\right.$ ) were added to dry toluene (50 $\mathrm{mL}$ ) and heated at reflux with stirring under an atmosphere of dry nitrogen for $20 \mathrm{hrs}$. The reaction mixture was allowed to cool to room temperature then solvents were removed under reduced pressure. The residue was first chromatographed on silica gel eluting with chloroform. Product containing fractions were combined and the solvents removed under reduced pressure. The residue was subsequently further purified by gel permeation chromatography on BioBeads SX-1 eluting with tetrahydrofuran giving the 
product as a dark green solid after removal of solvents. Yield: $28 \mathrm{mg}, 28 \%$. UV/Vis $\left(\mathrm{CH}_{2} \mathrm{Cl}_{2}\right): \lambda_{\max }=525.5,258.0 \mathrm{~nm} .{ }^{1} \mathrm{H}-\mathrm{NMR}\left(\mathrm{CDCl}_{3}, 55^{\circ} \mathrm{C}, 300 \mathrm{MHz}\right): \delta=1.13,1.15$ (overlapping s, 36H, $\left.{ }^{t} \mathrm{Bu}-\mathrm{H}\right), 1.33\left(\mathrm{~s}, 36 \mathrm{H},{ }^{t} \mathrm{Bu}-\mathrm{H}\right), 2.79\left(\mathrm{~s}, 6 \mathrm{H}, \mathrm{N}-\mathrm{CH}_{3}\right), 4.25\left(\mathrm{~d},{ }^{2} \mathrm{~J}=9.37\right.$ $\mathrm{Hz}, 2 \mathrm{H}$, pyrrolidine-H), 4.53 (s, 4H, benzyl $\left.-\mathrm{CH}_{2}-\right), 4.93(\mathrm{~s}, 2 \mathrm{H}$, pyrrolidine- $\mathrm{H}), 4.97(\mathrm{~d}$, ${ }^{2} \mathrm{~J}=9.54 \mathrm{~Hz}, 2 \mathrm{H}$, pyrrolidine-H), $6.54(\mathrm{~s}, 4 \mathrm{H}, \beta$-pyrrole- $\mathrm{H}), 6.84\left(\mathrm{~d},{ }^{3} \mathrm{~J}=7.89 \mathrm{~Hz}, 4 \mathrm{H}\right.$, biphenylyl-H), 6.91 (s, 4H cyclohexa-2,5-dienyl-H), 7.03 (d, ${ }^{3} J=4.40 \mathrm{~Hz}, 4 \mathrm{H}, \beta$-pyrroleH), 7.35 (d, ${ }^{3} \mathrm{~J}=8.07 \mathrm{~Hz}, 4 \mathrm{H}$, biphenylyl-H), 7.46 (d, ${ }^{3} \mathrm{~J}=7.87 \mathrm{~Hz}, 4 \mathrm{H}$, biphenylyl-H), 7.59 (s, 4H cyclohexa-2,5-dienyl-H), 7.81 (d, ${ }^{3} \mathrm{~J}=7.70 \mathrm{~Hz}, 4 \mathrm{H}$, biphenylyl-H), 9.21 (br. s, $2 \mathrm{H}$, pyrrole $\mathrm{NH}$ ) ppm. The bis- $\mathrm{C}_{60}$-adduct is too insoluble to obtain ${ }^{13} \mathrm{C}-\mathrm{NMR}$ data. FTIR: $v=2951.1$ (m, C-H str.), 2920.6 (m, C-H str.), 2860.4 (m, C-H str.), 2779.8 (w, C-H str.), 1637.3 (w, C=C str.), 1597.5 (s, C=C str.), 1538.9 (w), 1492.6 and 1485.4 (s, ${ }^{\mathrm{t}} \mathrm{Bu}$, asym. def.), 1461.2 and 1453.1 (s, 'Bu, sym. bend.), 1419.4 (w), 1404.9 (w), 1387.5 (m), 1360.2 (s, 'Bu, sym. bend.), $1333.0(\mathrm{~m}), 1307.8(\mathrm{~m}), 1257.4(\mathrm{~m}), 1230.5(\mathrm{w}), 1204.6(\mathrm{w})$, $1178.5(m), 1106.9(w), 1087.7(m), 1027.9(s), 1006.2(m), 946.9(m), 930.1(w), 885.9$ $(\mathrm{m}), 865.6(\mathrm{w}), 840.2(\mathrm{~m}), 816.8(\mathrm{~m}) \mathrm{cm}^{-1}$. MALDI-TOF-MS positive ion mode (dithranol): $[\mathrm{M}+2 \mathrm{H}]^{+}\left(\mathrm{C}_{228} \mathrm{H}_{124} \mathrm{~N}_{6} \mathrm{O}_{4}\right):$ calcd. 3011.97; found 3012.14. MALDI-TOF-MS negative ion mode (dithranol/TnBAF): $[\mathrm{M}+\mathrm{F}]^{-}\left(\mathrm{C}_{228} \mathrm{H}_{122} \mathrm{~N}_{6} \mathrm{O}_{4} \mathrm{~F}\right)$ : calcd. 3028.96; found 3029.47.

\subsubsection{Results and Discussions}

\subsubsection{Optical Studies}

Synthesis of bis(4-bromobenzyl)-substituted-OXP ${ }^{188,189}$ was followed by SuzukiMiyaura coupling with 4-formylphenylboronic acid to yield the bisformyl compound. ${ }^{190}$ The latter was used as a substrate for the Prato reaction ${ }^{10}$ yielding a $\operatorname{OXP}\left(\mathrm{C}_{60}\right)_{2}$ triad. 
Figure 5.1.1a shows the optical absorption spectral changes of $\operatorname{OXP}\left(\mathrm{C}_{60}\right)_{2}$ triad during increasing addition of $\mathrm{F}^{-}$in $\mathrm{o}$-dichlorobenzene (DCB). The main peak of $\operatorname{OXP}\left(\mathrm{C}_{60}\right)_{2}$ triad corresponding to OXP entity located at $511 \mathrm{~nm}$ revealed diminished intensity with the concurrent appearance of two new bands at 603 and $756 \mathrm{~nm}$.
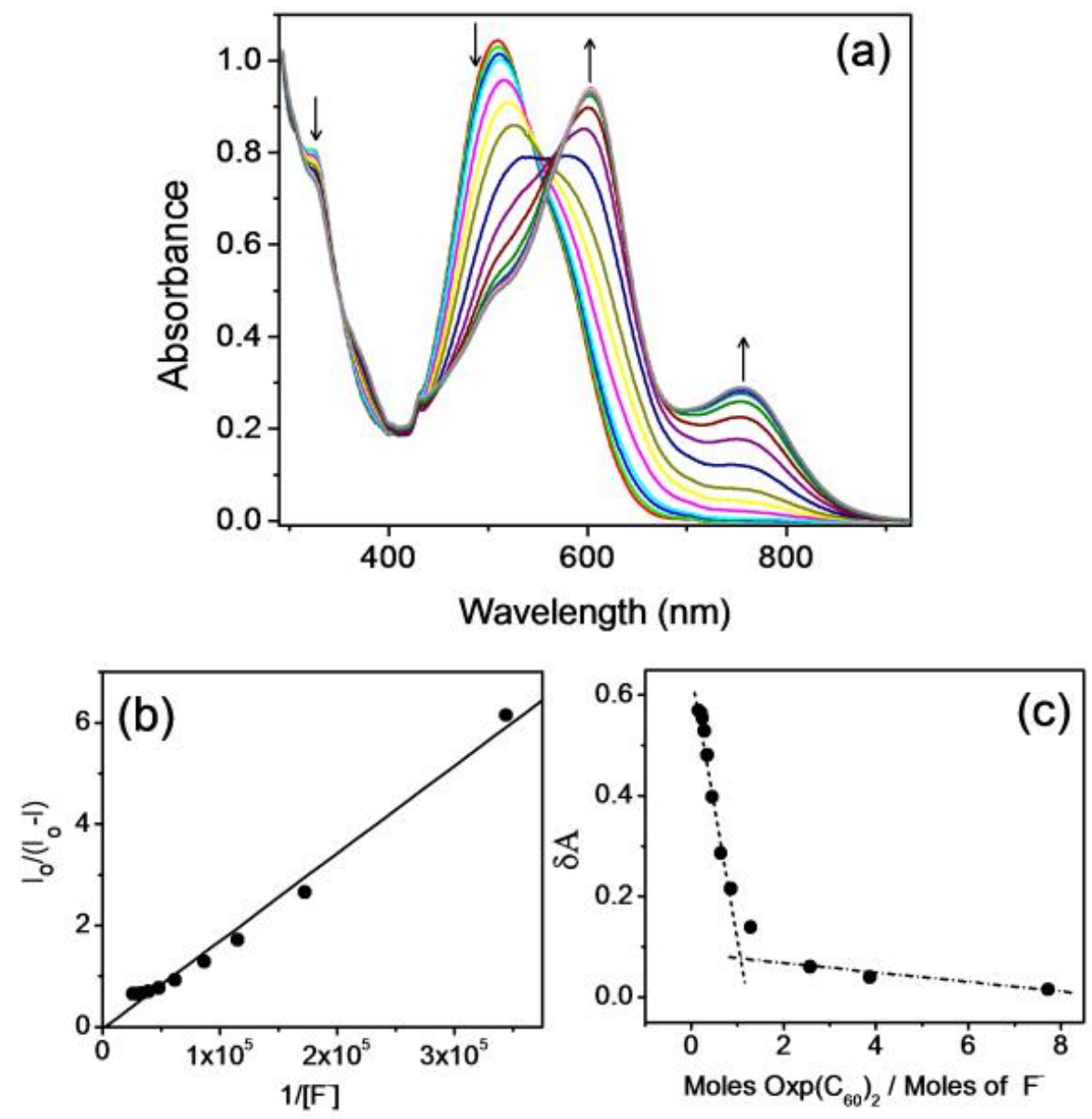

Figure 5.1.1: (a) Absorption spectra of $\operatorname{OXP}\left(\mathrm{C}_{60}\right)_{2}$ triad $(7.5 \mu \mathrm{M})$ upon increasing addition of $\mathrm{F}^{-}(0.1-0.5$ eq.). (b) Benesi-Hildebrand plot constructed to obtain the binding constant, and (c) mole ratio plot to obtain the molecular stoichiometry of the $\operatorname{OXP}\left(\mathrm{C}_{60}\right)_{2}$ triad: $\mathrm{F}^{-}$complex in DCB.

Isosbestic points at 309, 348 and $426 \mathrm{~nm}$ are observed suggesting the existence of only one equilibrium process in solution. The sharp peak at $430 \mathrm{~nm}$ of fulleropyrrolidine had no visible effect upon addition of $\mathrm{F}^{-}$in solution. Plots of mole ratio against absorbance change (Figure. 5.1.1c) suggest 1:1 complex formation. The binding constant evaluated 
by constructing Benesi-Hildebrand plot $^{90}$ (Figure. 5.1.1b) is found to be $5.8 \times 10^{4} \mathrm{M}^{-1}$, suggesting stable complex formation.

Both $\mathrm{OXPR}_{2}(\mathrm{R}=\text { alkyl or aryl })^{187}$ and fulleropyrrolidine $\mathrm{e}^{92,179,181}$ are known to emit in the $720 \mathrm{~nm}$ region and this is also the case for the investigated compound $\operatorname{OXP}\left(\mathrm{C}_{60}\right)_{2}$ triad, albeit with much lower quantum yields. That is, a broad emission spanning the $600-850 \mathrm{~nm}$ range was observed for $\operatorname{OXP}\left(\mathrm{C}_{60}\right)_{2}$ triad (Figure. 5.1.2a). The weak emission of fullerene is hidden under the relatively strong emission of OXP. Addition of $\mathrm{F}^{-}$further decreased the emission intensity resulting in formation of an almost non-emitting complex (>98\% quenching) likely caused by occurrence of very efficient photochemical events.
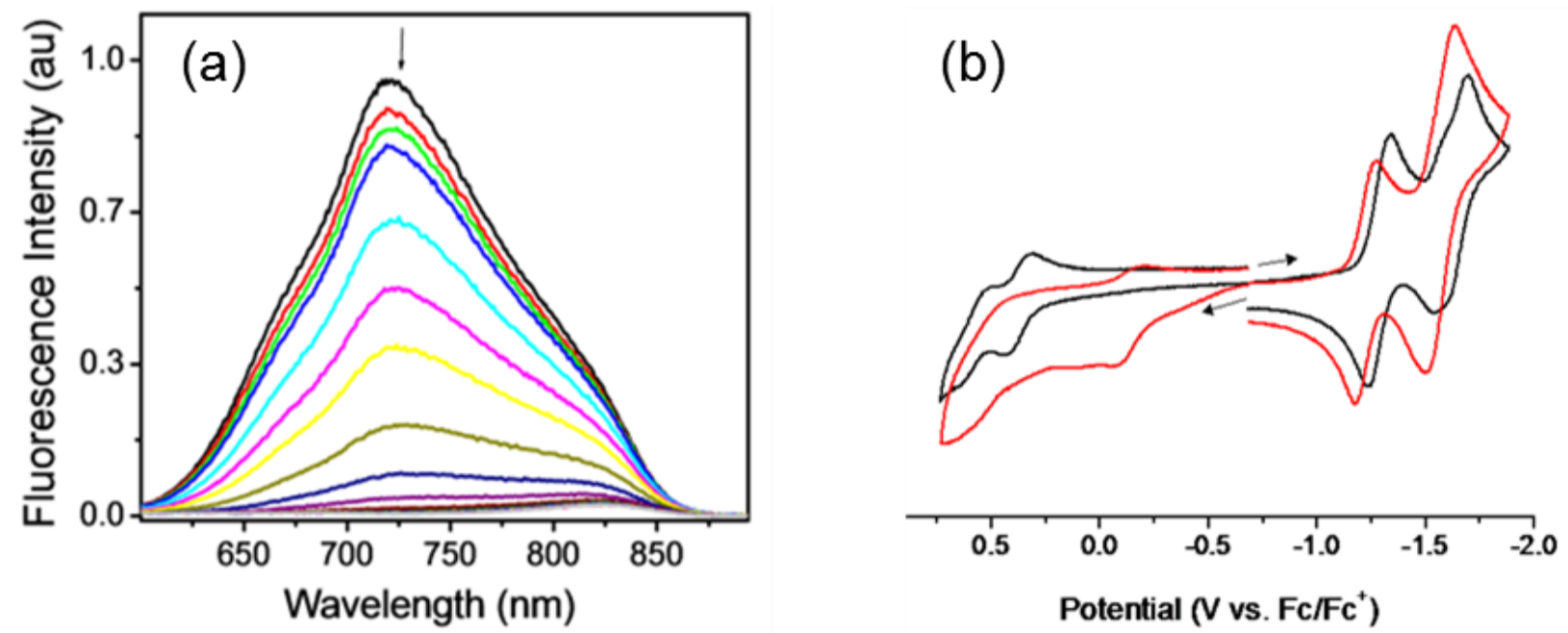

Figure 5.1.2: (a) Fluorescence spectra of $\operatorname{OXP}\left(\mathrm{C}_{60}\right)_{2}$ triad $(7.5 \mu \mathrm{M})$ on increasing addition of $\mathrm{F}^{-}\left(0.1\right.$ - 0.5 eq.) in DCB. $\lambda_{\mathrm{ex}}=509 \mathrm{~nm}$. (b) Cyclic voltammograms of $\operatorname{OXP}\left(\mathrm{C}_{60}\right)_{2}$ triad in the absence (dark line) and presence (red line) of $\mathrm{F}^{-}$in deaerated DCB containing $0.1 \mathrm{M}(\mathrm{TBA}) \mathrm{ClO}_{4}$. Scan rate $=100 \mathrm{mV} / \mathrm{s}$.

\subsubsection{Electrochemical Studies}

Electrochemical studies are performed to probe the effect of anion binding on the redox potentials of $\operatorname{OXP}\left(\mathrm{C}_{60}\right)_{2}$ triad and their subsequent effect on the energy levels of photoinduced processes. The first oxidation of $\operatorname{OXP}\left(\mathrm{C}_{60}\right)_{2}$ triad is a reversible process 
located at $E_{1 / 2}=0.37 \mathrm{~V}$ vs. $\mathrm{Fc} / \mathrm{Fc}^{+}$in $\mathrm{DCB}$ as shown in the Figure 5.1.2b. The potential value is similar to that reported earlier for $\operatorname{OXP}(b z)_{2}\left(b z=\right.$ benzyl) derivative ${ }^{191}$ lacking the fullerene suggesting that the site of oxidation involves the OXP unit. A second oxidation is also observed at higher anodic potentials $\left(E_{1 / 2}=0.58 \mathrm{~V}\right)$. The fullerene reduction of $\operatorname{OXP}\left(\mathrm{C}_{60}\right)_{2}$ triad is located at $E_{1 / 2}=-1.28 \mathrm{~V}$ vs. $\mathrm{Fc} / \mathrm{Fc}^{+}$, whereas the first reduction of OXP and the second reduction of fullerene are close and appeared at -1.56 and $-1.64 \mathrm{~V}$, respectively. Interestingly, addition of $\mathrm{F}^{-}$revealed drastic changes in the oxidation potentials of the OXP entity. That is, addition of 1.1 eq. of $\mathrm{F}^{-}$to a solution of $\operatorname{OXP}\left(\mathrm{C}_{60}\right)_{2}$ triad resulted in a cathodic shift of nearly $510 \mathrm{mV}\left(E_{1 / 2}=-0.14 \mathrm{~V}\right)$ as a result of $\mathrm{F}^{-}$binding to the OXP unit. On the reduction side, the fullerene reduction underwent a small anodic shift of $60 \mathrm{mV}\left(E_{1 / 2}=-1.22 \mathrm{~V}\right)$ due to ion-pairing effect. ${ }^{192}$ However, no appreciable shifts are seen for OXP reduction. Free-energy calculations for charge separation (CS) and charge recombination (CR) were performed according to Weller's approach. ${ }^{179}$ The driving forces for charge recombination $\left(-\Delta G_{C R}\right)$ and charge separation $\left(-\Delta G_{C S}\right)$ processes of $\operatorname{OXP}\left(C_{60}\right)_{2}$ triad via the singlet excited state of OXP and $\mathrm{C}_{60}$ are evaluated as 1.65 and $0.10 \mathrm{eV}$, respectively, taking into consideration that the energy of the singlet excited state of OXP and $\mathrm{C}_{60}$ are $1.75 \mathrm{eV} .{ }^{15,187}$ In case of $\operatorname{OXP}\left(\mathrm{C}_{60}\right)_{2}$ triad: $\mathrm{F}^{-}$, the $-\Delta \mathrm{G}_{\mathrm{CR}}$ value was determined to be $1.14 \mathrm{eV}$. The PET processes are exothermic from the singlet $\left(-\Delta G_{C S}=0.61 \mathrm{eV}\right)$ and triplet $\left(-\Delta G_{C S}=0.41 \mathrm{eV}\right)$ excited states of the OXP unit.

\subsubsection{Transient Absorption Studies}

Spectroscopic signature for the formation of radical ion pair species and kinetics of CS and CR are obtained from the transient absorption studies in the femtosecond 
and nanosecond time regimes by utilizing the $430 \mathrm{~nm}$ laser light, which selectively excites the OXP entity (Figure 5.1.3).

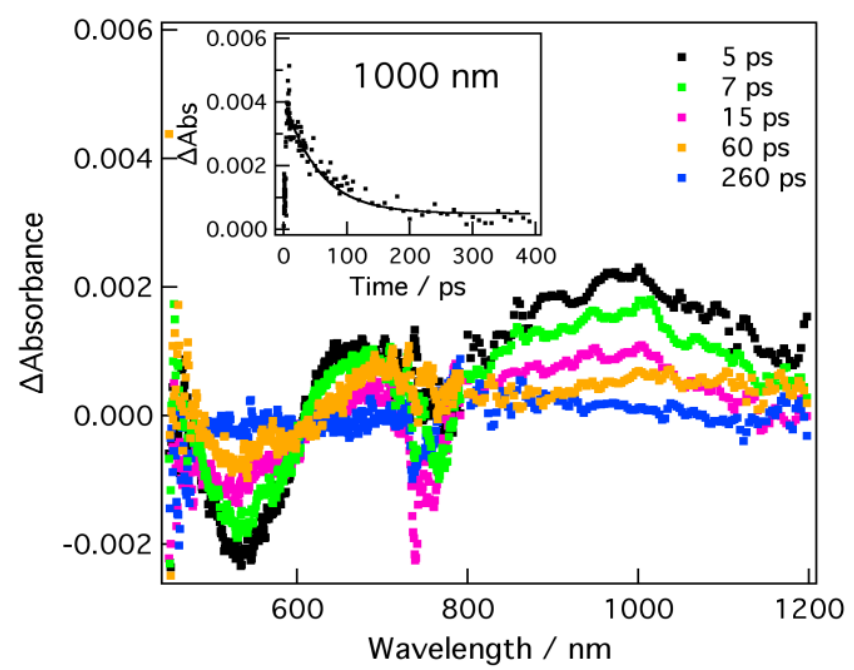

Figure 5.1.3: Femtosecond transient absorption spectra of $\operatorname{OXP}\left(\mathrm{C}_{60}\right)_{2}$ triad: $\mathrm{F}^{-}$in deaerated DCB. $\lambda_{\mathrm{ex}}=430 \mathrm{~nm}$. The figure inset shows decay of the $1000 \mathrm{~nm}$ transient band corresponding to fullerene radical anion.

The femtosecond transient absorption spectrum of $\operatorname{OXP}\left(\mathrm{C}_{60}\right)_{2}$ triad and $\operatorname{OXP}\left(\mathrm{C}_{60}\right)_{2}$ triad: $\mathrm{F}^{-}$revealed absorption bands in the visible and near infrared (NIR) regions with maxima at 870 and $1000 \mathrm{~nm}$ due to $\mathrm{OXP}^{*+}$ and $\mathrm{C}_{60}{ }^{-}$species, respectively, offering clear evidence for the occurrence of PET from the electron donating OXP to the electron accepting $\mathrm{C}_{60 .}{ }^{186,193}$ The rate of CS via the singlet excited state is found to be very fast $\left(\sim 10^{12} \mathrm{~s}^{-1} \mathrm{ps}\right)$. From fitting the decay of the radical ion pair species with clean first-order kinetics, the rates of charge recombination $\left(k_{\mathrm{CR}}\right)$ were found to be $6.6 \times 10^{10}$ and $1.40 \times 10^{10} \mathrm{~s}^{-1}$ for $\operatorname{OXP}\left(\mathrm{C}_{60}\right)_{2}$ triad and $\operatorname{OXP}\left(\mathrm{C}_{60}\right)_{2}$ triad: $\mathrm{F}^{-}$, respectively. Using these $k_{\mathrm{CR}}$ values, lifetimes of the charge-separated states $\left(\tau_{\mathrm{CS}}=1 / k_{\mathrm{CR}}\right)$ via the singlet state were calculated to be 15 and 72 ps for $\operatorname{OXP}\left(\mathrm{C}_{60}\right)_{2}$ triad and $\operatorname{OXP}\left(\mathrm{C}_{60}\right)_{2}$ triad: $\mathrm{F}^{-}$, respectively, revealing the effect of $\mathrm{F}^{-}$on stabilization of the charge-separated state. 

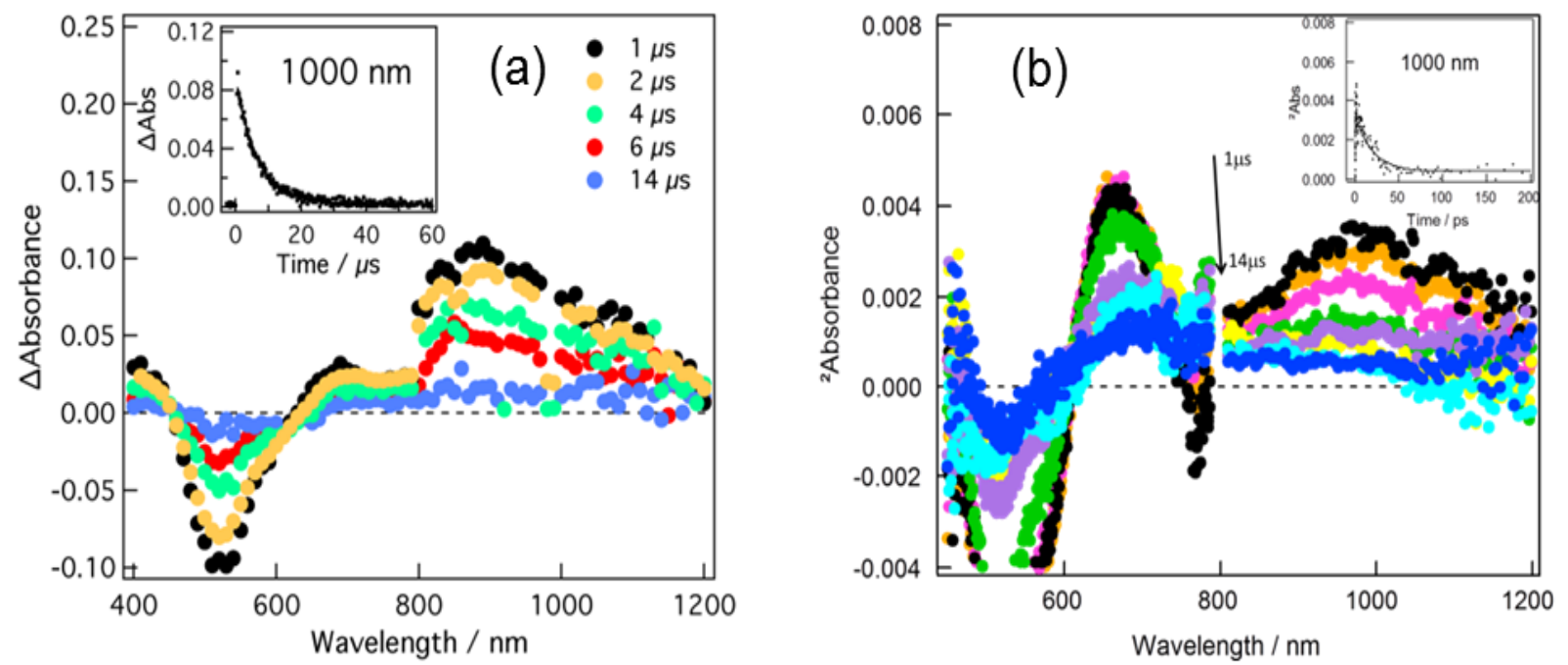

(c)

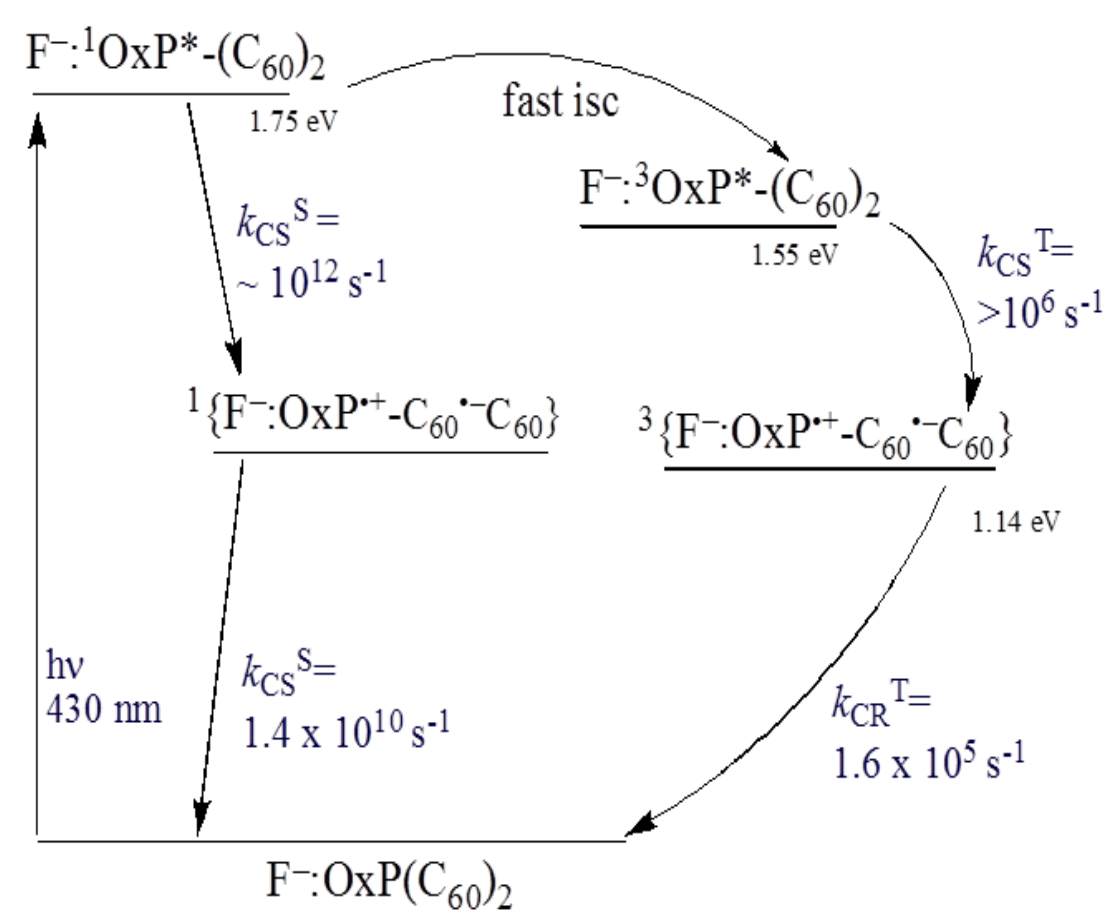

Figure 5.1.4: (a) Nanosecond transient absorption spectra of $\operatorname{OXP}\left(\mathrm{C}_{60}\right)_{2}$ triad: $\mathrm{F}^{-}$in deaerated DCB. Inset shows the time profile of the $\mathrm{C}_{60^{\circ}}{ }^{-}$at $1000 \mathrm{~nm}$. (b) Nanosecond transient absorption spectra obtained by $430 \mathrm{~nm}$ laser light of 1 in deaerated DCB.inset shows time profile of $\mathrm{C}_{60}{ }^{-}$band at $1000 \mathrm{~nm}$. (c) Energy level diagram showing the electron transfer processes of $\operatorname{OXP}\left(\mathrm{C}_{60}\right)_{2}$ triad: $\mathrm{F}^{-}$via the singlet and triplet $\mathrm{OXP}$ in deaerated DCB. 
Nanosecond transient absorption spectral studies provided evidence of PET from the triplet excited state of the OXP unit, taking into account the thermodynamic feasibility of electron transfer of $\operatorname{OXP}\left(\mathrm{C}_{60}\right)_{2}$ triad: $\mathrm{F}^{-}$via the triplet state of $\mathrm{OXP}$, as mentioned earlier. As shown in Fig. 5.1.4a, the transient absorption spectrum exhibited peaks at 860 and $1000 \mathrm{~nm}$ due to $\mathrm{OXP}^{*+}$ and $\mathrm{C}_{60}{ }^{-{ }^{-}}$species. ${ }^{186,193}$ The rate of CS from the triplet excited state was too fast to detect within the time resolution of the nanosecond laser pulse. By monitoring the decay of the $\mathrm{C}_{60}$ radical anion band, $k_{\mathrm{CR}}{ }^{\top}$ was determined as $1.6 \times 10^{5} \mathrm{~s}^{1}$. Based on $k_{\mathrm{CR}}{ }^{\top}$, the lifetime of the triplet CS state was evaluated as (6.3 $\mu \mathrm{s})$, which is a relatively long-lived species compared to the widely investigated porphyrin-fullerene dyads in the literature. ${ }^{15,47,92,152,156,166,179-182}$ The photochemical events via the singlet and triplet excited states of OXP are summarized as shown in Figure 5.1.4c.

\subsubsection{Summary}

We have synthesized a novel molecular triad comprised of a redox-active anionreceptor (OXP) and $\mathrm{C}_{60}$ entities. The OXP entity binds $\mathrm{F}^{-}$through the imino-hydrogens leading to a large cathodic shift of its oxidation potential. As a result, the donor-acceptor pair reveals very efficient PET from both the singlet and triplet excited states of OXP. Presence of $\mathrm{F}^{-}$in the OXP pocket slows down the CR process thus generating the much desired long-lived CS state. 


\subsection{Anion-Complexation-Induced Stabilization of Charge Separation ${ }^{\S}$}

\subsubsection{Introduction}

Complex oligochromophoric molecular systems containing porphyrins are essential specimens for study of a variety of photoinduced reactions. ${ }^{11,194-200}$ Thus, many chromophoric compounds have been prepared and analyzed for the lifetimes of their charge-separated (CS) states, ${ }^{17,97,201-211}$ which have relevance to photosynthesis, or for their photonic applications. ${ }^{14,181,212-214}$ Molecular donor-acceptor systems composed of tetrapyrroles (porphyrins) and electron acceptors such as fullerenes have attracted special attention. ${ }^{94,215-227}$ In certain cases, activity of naturally occurring systems is optimum only in the presence of an ionic cofactor either due to resulting structural stabilization ${ }^{228-231}$ or because of charge-balancing by the ion..$^{232-234}$ This situation can be referred to as heterotropic allosteric regulation and is observed in many enzyme systems using organic or inorganic ions. For example, Nature uses chloride anions as a cofactor of the oxygen-evolving complex (OEC). ${ }^{184,235-241}$ In order to accommodate both reagent(s) and cofactor(s) in locations appropriate for their functions it is necessary that a biological enzyme or synthetic species should possess at least two different binding sites.

Inspired by the above concept, here, we have built a supramolecular oligochromophoric model system containing sites for binding of a reagent species and an anionic species in order to probe the effect of the binding of an anionic cofactor on the identities of products from photoinduced processes that occur within the resulting

${ }^{\S}$ Section 5.2 in its entirety is reproduced from the Journal of American Chemical Society, 2009, 131 (44), pp 16138- 16146 with permission from the publisher. 
complex. This is important because investigation of the identity and stability of photoinduced CS complexes provides insights into methods for modulating potentially useful electronic properties of synthetic systems.

The concept of the dual binding mode of compound $\mathbf{1}$ is illustrated in Figure 5.2.1. The bis-porphyrin-substituted oxoporphyrinogen 1 (Figure 5.2.1b) has two different binding sites as required. One site (composed of two porphinatozinc(II) units) capable of binding bis(4-pyridyl)-substituted guests through coordination to the central zinc cations ${ }^{187}$ while the other site (composed of two pyrrole-type amine groups of the oxoporphyrinogen unit) interacts with anionic species through hydrogen bonding. ${ }^{188}$ The anion binding site, oxoporphyrinogen (OXP) unit, prepared by the 2-electron oxidation of tetrakis(3,5-di-t-butyl-4-hydroxyphenyl)porphyrin, can be multiple substituted at central nitrogen atoms in a stepwise and regioselective way permitting the synthesis of $\mathbf{1} .^{191,242}$
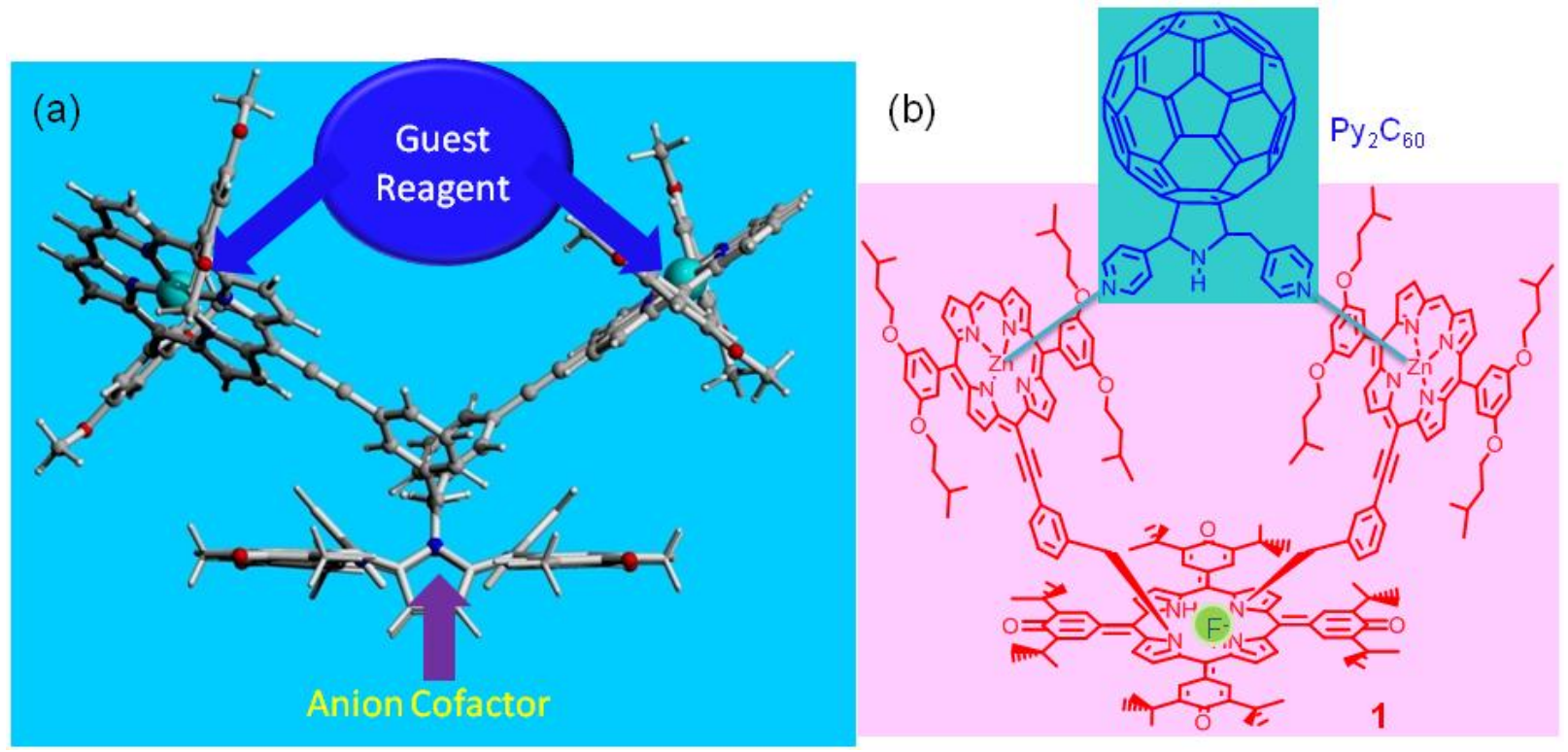

Scheme 5.2.1: Structural origin of two-guest reagent/anion cofactor complexation (left figure, peripheral substituents are omitted for clarity) and chemical structure of the bisporphyrin- substituted oxoporphyrinogen, 1 and bis(4-pyridyl)-substituted fullerene used in this work. Axial coordination and anion binding site are also shown for clarity 
The effect of anion binding on the intramolecular photoinduced electron-transfer processes has been probed by the femtosecond and nanosecond laser flash photolysis measurements of the supramolecular complex between 1 and $\mathrm{Py}_{2} \mathrm{C}_{60}$ in the absence and presence of different anions. As demonstrated, complexation of anions to the oxoporphyrinogen center lowers its oxidation potential by nearly $600 \mathrm{mV}$ creating an intermediate energy state for charge migration from the $\mathrm{ZnP}^{\circ+}$ to the oxoporphyrinogen:anion complex. A decrease in charge recombination (CR) driving force caused by anion binding combined with an increase in reorganizational energy of electron transfer results in the desired increase in the lifetime of the CS state. The present study provides valuable insight into the role of anion binding on the control of the photodynamics of both photoinduced electron transfer and charge recombination reactions.

\subsubsection{Experimental Section}

Chemicals

The following synthesis was done by students in Prof. Jonathan Hill's lab. The syntheses of OXP-(ZnP $)_{2}, 1$ and fullerene were carried out according our earlier published methods. ${ }^{126,187}$

\subsubsection{Results and Discussion}

\subsubsection{Optical Studies - Formation and Characterization of the Two-Host Bound} Supramolecular Guest Complex

The formation of two-host bound supramolecular guest system was followed in two steps, viz., $\mathrm{Py}_{2} \mathrm{C}_{60}$ binding to the host system, 1 to form 1.Py $\mathrm{C}_{60}$, and binding of anion to the OXP anion binding site to yield $\mathbf{1}\left(\mathrm{F}^{-}\right) \cdot \mathrm{Py}_{2} \mathrm{C}_{60}$ supramolecular complex. Optical 
absorption and $\mathrm{H}^{1} \mathrm{NMR}$ studies were systematically performed to arrive at the stoichiometry and binding constants of the two-host bound complex. Electronic spectral changes during binding of $\mathrm{Py}_{2} \mathrm{C}_{60}$ to 1 are shown in Figure 5.2.1a. These spectra illustrate complexation of $\mathrm{Py}_{2} \mathrm{C}_{60}$ with zinc porphyrin ${ }^{126}$ as revealed by the red shift of absorbance bands of porphyrinatozinc of 1 located at 439, 559 and 601 to 443,571 and $621 \mathrm{~nm}$, respectively. The unique topology of 1 with a $V$-alignment of the porphyrin macrocycles allowed supramolecular complex formation by adopting the wellestablished 'two-point' coordinative binding of bis pyridine functionalized fullerene. ${ }^{187}$ The binding constant calculated from Benesi-Hildebrand method ${ }^{90}$ was found to be $1.9 \mathrm{x}$ $10^{5} \mathrm{M}^{-1}$ (Figure 5.2.1a inset). This value is nearly two orders of magnitude higher compared to mono pyridine fulleropyrrolidine binding to zinc porphyrin ${ }^{128}$ mainly due to the cooperative effect induced by the bis pyridine functionalized fullerene to appropriately positioned zinc porphyrin macrocycles. ${ }^{126,187}$ Job's plot confirmed 1:1 stoichiometry of the 1. $\mathrm{Py}_{2} \mathrm{C}_{60}$ complex. It is important to note that the oxoporphyrinogen band located at $513 \mathrm{~nm}$ exhibited no shift in the band position suggesting lack of direct interaction with $\mathrm{ZnP}$ or $\mathrm{Py}_{2} \mathrm{C}_{60}$. It may also be mentioned here that the geometry of 1.Py $\mathrm{C}_{2} \mathrm{C}_{60}$ deduced from B3LYP/3-21G $\left(^{*}\right)$ method yielded a stable structure on BornOppenheimer potential energy surface. ${ }^{187}$ 

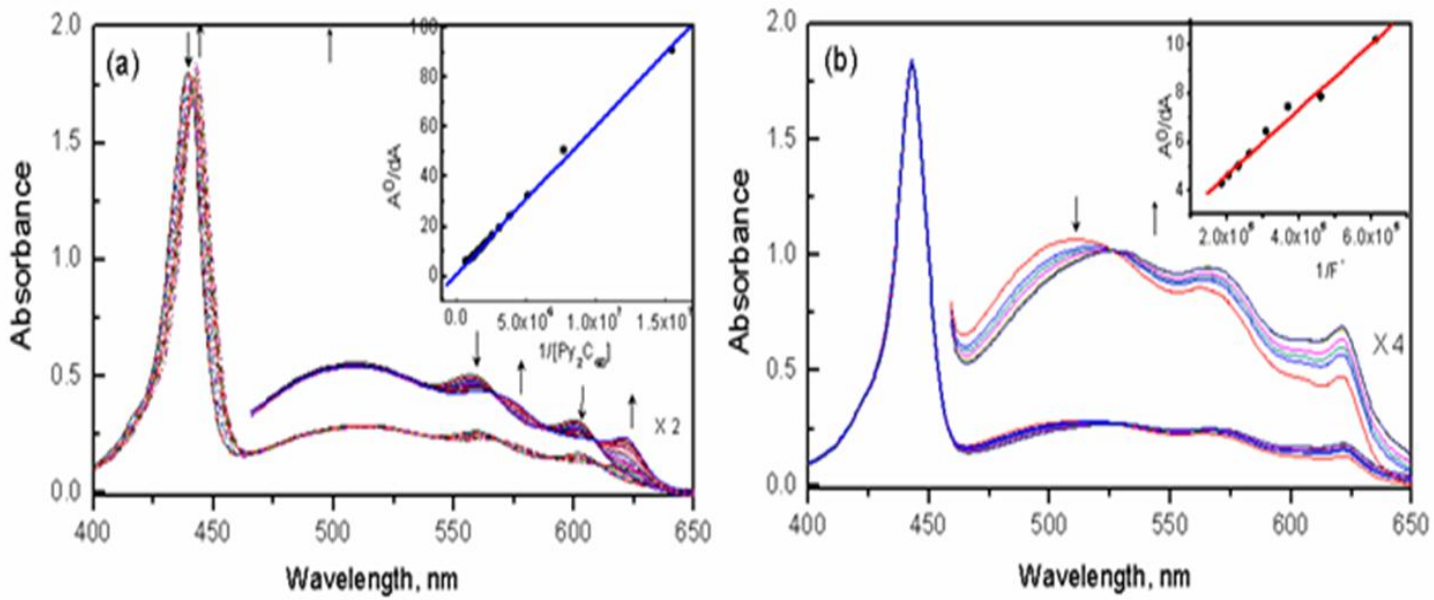

Figure 5.2.1: Optical absorption spectral changes observed during (a) binding of $\mathrm{Py}_{2} \mathrm{C}_{60}$ to 1 to form 1. $\mathrm{Py}_{2} \mathrm{C}_{60}$ complex, and (b) $\mathrm{F}$ - binding to OXP of the $1 \mathrm{Py}_{2} \mathrm{C}_{60}$ complex to form the $1\left(\mathrm{~F}^{-}\right)$. $\mathrm{Py}_{2} \mathrm{C}_{60}$ complex in o-dichlorobenzene. The figure insets show BenesiHildebrand plots constructed to obtain the binding constants; $A_{\circ}$ and $d A$ represent respectively absorbance values in the absence of added guest and change in absorbance upon guest binding.

The anion binding to the OXP anion binding site was subsequently performed as shown for $\mathrm{F}^{-}$binding to $1 . \mathrm{Py}_{2} \mathrm{C}_{60}$ complex in Figure 5.2.1b. The fluoride anion binding to the oxoporphyrinogen indicated by the red shift of its absorbance band from $513 \mathrm{~nm}$ to $530 \mathrm{~nm} .{ }^{188}$ Notably, no further shifts of the porphyrin absorbance bands occur on addition of fluoride anions to a solution of the 1.Py $\mathrm{C}_{60}$ complex indicating that fluoride anions do not interact with the porphyrinatozinc entities. This is due in part to the preferential binding of $\mathrm{F}^{-}$to OXP as revealed by the higher $K$ values, and strong binding of $\mathrm{Py}_{2} \mathrm{C}_{60}$ to the porphyrinatozinc moieties through the two-point binding motif ${ }^{187}$ which maintains the guest exclusivity of the two binding sites. The binding constant calculated was found to be (Figure 5.2.1b inset) $7.4 \times 10^{4} \mathrm{M}^{-1}$ and the molecular stoichiometry from the Job's plot was found to be one equivalent of $\mathrm{F}^{-}$binding to the OXP pocket of the 1. $\mathrm{Py}_{2} \mathrm{C}_{60}$ complex. 

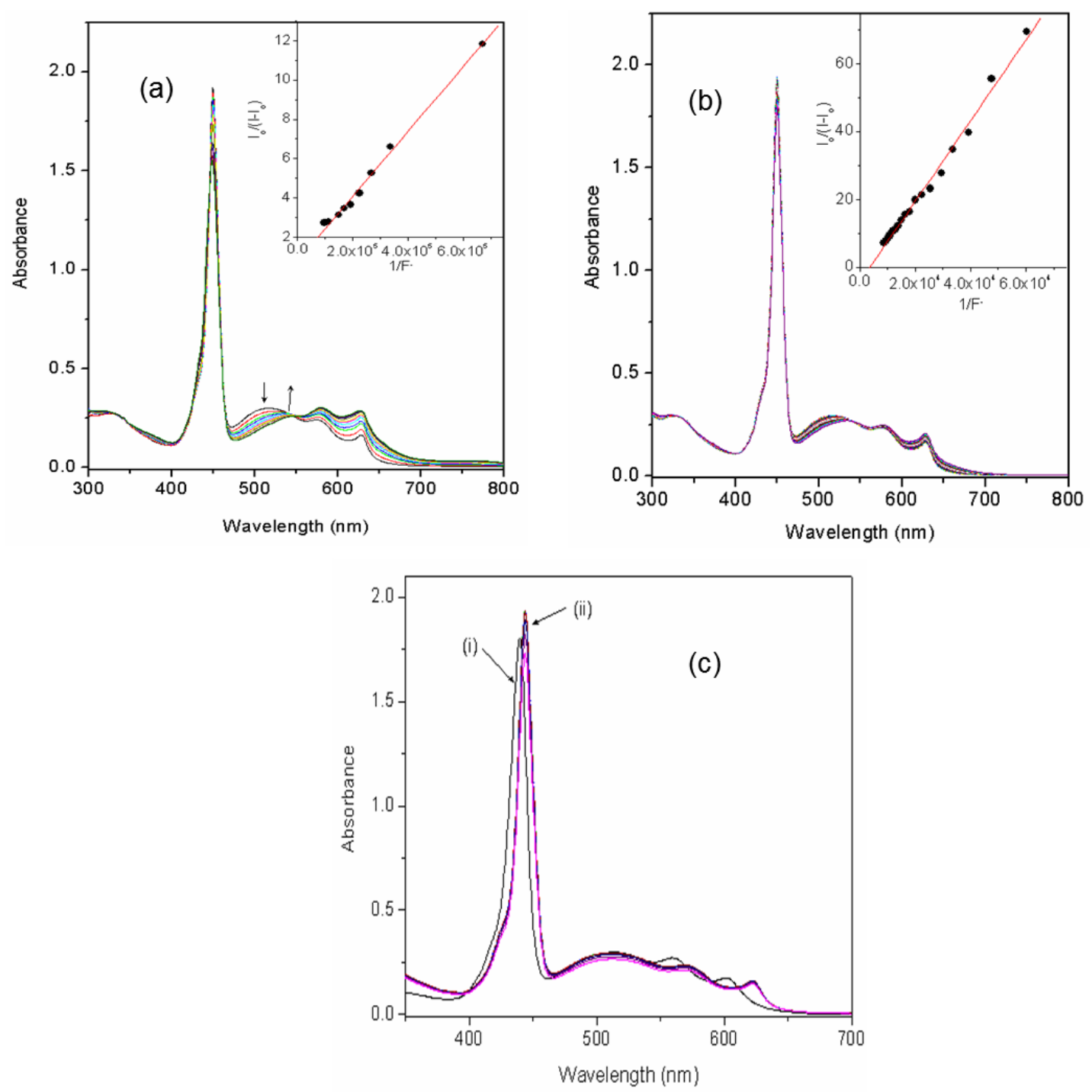

Figure 5.2.2: (a) Optical absorption spectral changes observed during acetate binding to $1-\mathrm{Py}_{2} \mathrm{C}_{60}$ to form the $1\left(\mathrm{CH}_{3} \mathrm{COO}^{-}\right)$. $\mathrm{Py}_{2} \mathrm{C}_{60}$ complex in o-dichlorobenzene.(b) Optical absorption spectral changes observed during dihydrogen phosphate binding to 1 . $\mathrm{Py}_{2} \mathrm{C}_{60}$ to form the $1\left(\mathrm{H}_{2} \mathrm{PO}^{-}\right)$. $\mathrm{Py}_{2} \mathrm{C}_{60}$ complex in o-dichlorobenzene. The figure inset shows a Benesi-Hildebrand plot constructed to obtain the binding constant. (c) Optical absorption spectral changes observed during perchlorate binding to 1 . $\mathrm{Py}_{2} \mathrm{C}_{60}$ to form the 1 (ClO4-). $\mathrm{Py}_{2} \mathrm{C}_{60}$ complex. Spectrum (i) represents that of 1 and (ii) represents that of 1. $\mathrm{Py}_{2} \mathrm{C}_{60}$ in o-dichlorobenzene. No change in the $513 \mathrm{~nm}$ band of OXP upon addition of $\mathrm{ClO}^{-}$indicate little or no interaction.

Similar spectral changes were observed for acetate and dihydrogen phosphate binding to 1 (see Figures 5.2.2a and 5.2.2b for spectral changes and Benesi-Hildebrand plots); the binding constants are given in Table 5.2.1. Addition of $\mathrm{ClO}_{4}{ }^{-}$to the 1.Py $\mathrm{C}_{60}$ complex solution caused no detectable spectral changes, suggesting little or no binding of this 
anion to the OXP (Figure 5.2.2c). The magnitude of the anion binding constants were in the same order of magnitude compared to the earlier reported values for anion binding to bis benzyl OXP (ZnP entities replaced with benzyl groups), abbreviated as $\mathrm{Ox}(\mathrm{bz})_{2}{ }^{188}$ Binding constants of thebis-(4-pyridyl)fullerene guest to 1 represent the binding of the mixture of diastereomers obtained at synthesis of $\mathrm{Py}_{2} \mathrm{C}_{60}$. The minor differences in geometry introduced to the host-guest complexes were consequently neglected.

Table 5.2.1: Binding constants $(K)$, rate constants of charge separation $\left(k_{c s}\right)$, charge recombination $\left(k_{\mathrm{CR}}\right)$ and lifetimes of CS states $\left(\tau_{\mathrm{RIP}}\right)$ for the investigated supramolecular complexes in o-dichlorobenzene

\begin{tabular}{|c|c|c|c|c|}
\hline Compound & $\boldsymbol{K}\left[\mathbf{M}^{-1}\right]$ & $\boldsymbol{k}_{\mathbf{C s}}{ }^{\mathbf{S}}\left[\mathbf{s}^{-1}\right]$ & $\boldsymbol{k}_{\mathbf{C R}}{ }^{\top}\left[\mathbf{s}^{-1}\right]$ & $\tau_{\text {RIP }}[\boldsymbol{\mu s}]$ \\
\hline $\mathbf{1}+\mathrm{Py}_{2} \mathrm{C}_{60}$ & $1.9 \times 10^{5}$ & $1.6 \times 10^{10}$ & $6.1 \times 10^{6}$ & 0.16 \\
\hline $\mathbf{1}: \mathrm{Py}_{2} \mathrm{C}_{60}+\mathrm{F}^{-}$ & $7.4 \times 10^{4}$ & $2.9 \times 10^{10}$ & $7.1 \times 10^{5}$ & 14 \\
\hline $\mathbf{1}: \mathrm{Py}_{2} \mathrm{C}_{60}+\mathrm{CH}_{3} \mathrm{COO}^{-}$ & $6.1 \times 10^{4}$ & $1.2 \times 10^{10}$ & $9.1 \times 10^{5}$ & 11 \\
\hline $\mathbf{1}: \mathrm{Py}_{2} \mathrm{C}_{60}+\mathrm{H}_{2} \mathrm{PO}_{4}^{-}$ & $8.4 \times 10^{2}$ & $1.5 \times 10^{10}$ & $1.3 \times 10^{5}$ & 7.5 \\
\hline $\mathbf{1}: \mathrm{Py}_{2} \mathrm{C}_{60}+\mathrm{ClO}_{4}^{-}$ & No binding & $1.7 \times 10^{10}$ & $5.8 \times 10^{6}$ & 0.17 \\
\hline
\end{tabular}

\subsubsection{NMR Studies}

Further evidence for the structure of the $\mathrm{F}^{-}$bound $1 . \mathrm{Py}_{2} \mathrm{C}_{60}$ complex was obtained by using ${ }^{1} \mathrm{H}$ NMR spectroscopy (see Figure 5.2.3). ${ }^{1} \mathrm{H}$ NMR spectra of 1 revealed gradual spectral changes during titration with up to one equivalent of $\mathrm{Py}_{2} \mathrm{C}_{60}$ in o-dichlorobenzene- $\mathrm{d}_{4}$ (Figure 5.2.3a). 
(a)

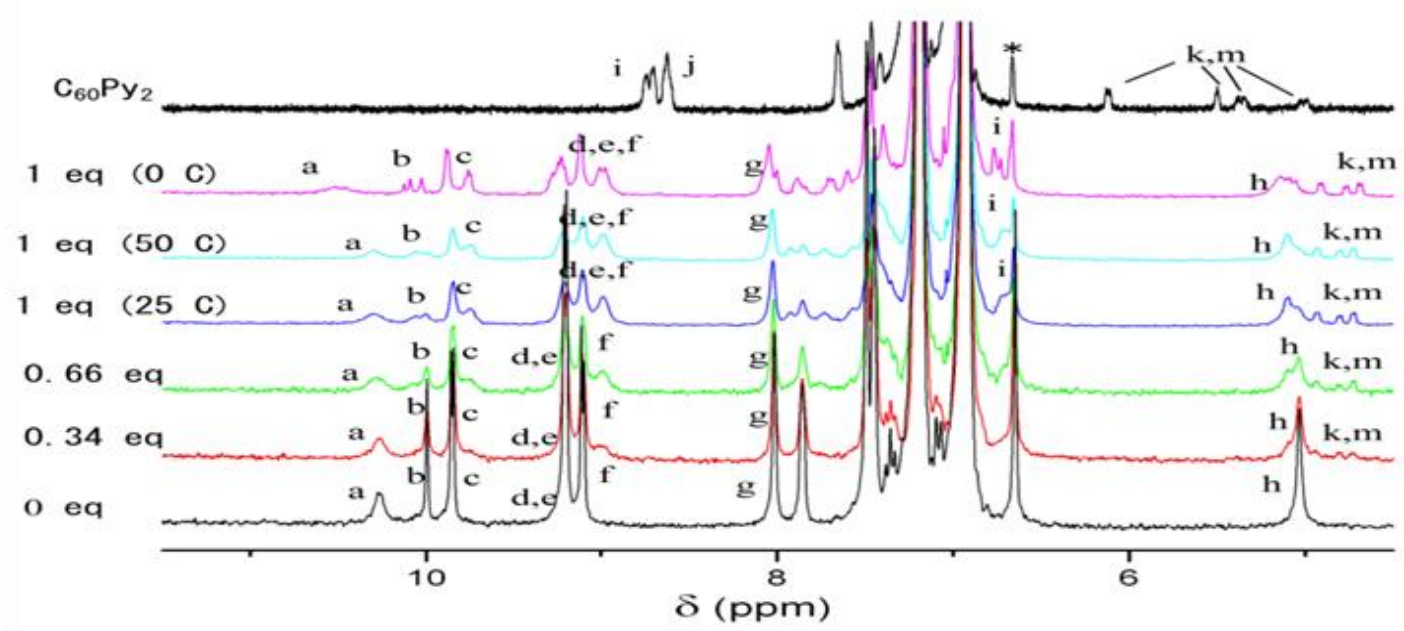

(b)
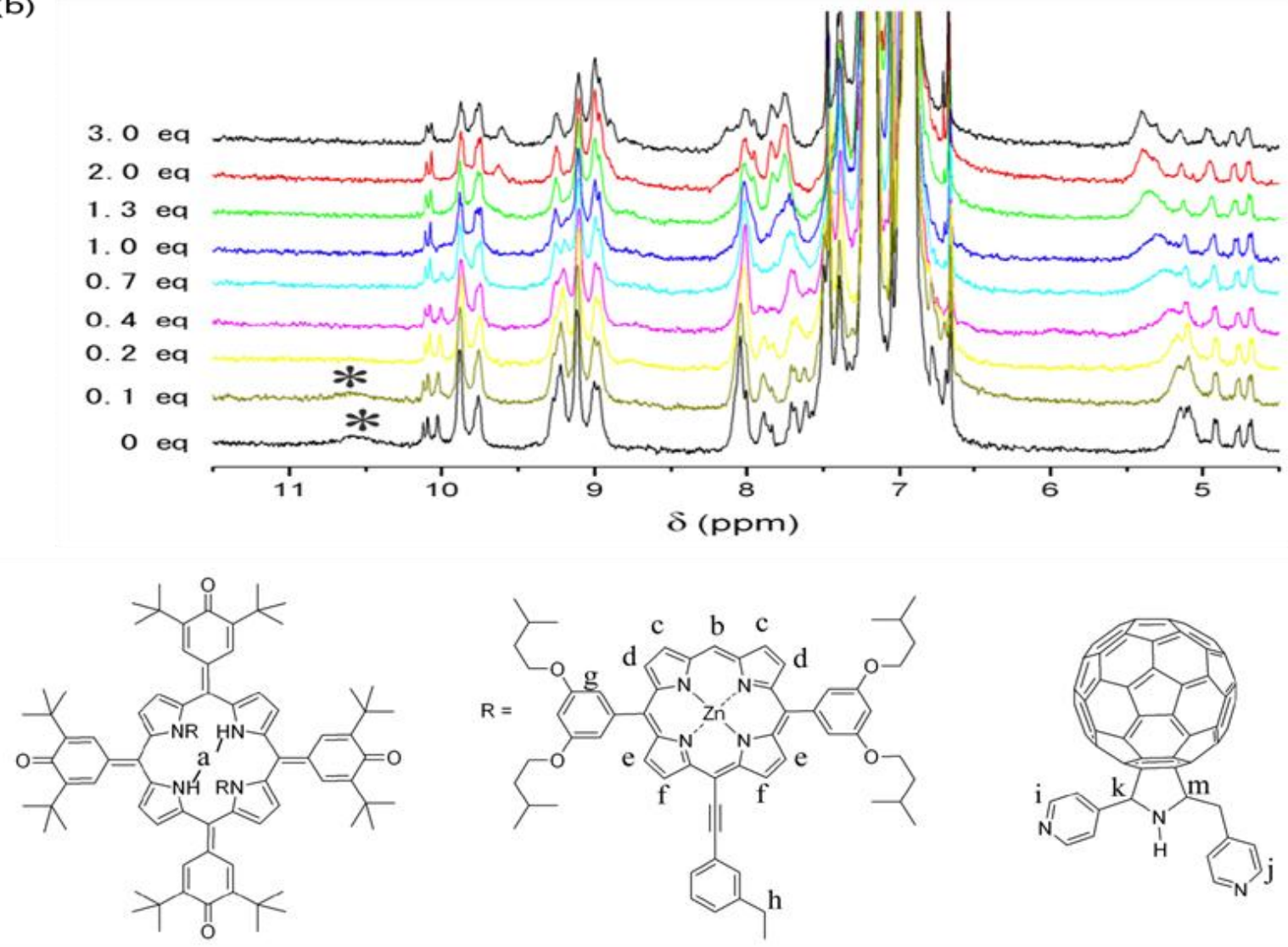

Figure 5.2.3: (a) ${ }^{1} \mathrm{H}$ NMR titration of 1 with up to 1 equivalent of $\mathrm{Py}_{2} \mathrm{C}_{60}$ in odichlorobenzene-d4. Note upfield shift of resonances of $\mathrm{Py}_{2} \mathrm{C}_{60}$ due to shielding by porphyrin aromatic m-electronic system. Assignments of relevant peaks are shown in the lower part of the figure. (b) Titration of the 1. $\mathrm{Py}_{2} \mathrm{C}_{60}$ complex formed in (a) with fluoride anions (tetra-n-butylammonium fluoride solution in o-dichlorobenzene-d4). Asterisk denotes porphyrinogen $\mathrm{NH}$ resonance. Note the disappearance of the peak labeled with an asterisk due to hydrogen bonding to fluoride anions. 
That is, the resonance due to the axially coordinated pyridyl protons experiences an upfield shift (of around $2 \mathrm{ppm}$ ) as a result of their proximity to the porphyrin aromatic system while shifts in the frequency of the resonances due to the $\mathrm{ZnP}$ or OXP entities were minimal. Addition of $\mathrm{Py}_{2} \mathrm{C}_{60}$ beyond one equivalent resulted in a gradual shift of the pyridyl proton resonances to their expected position due to exchange, suggesting a 1:1 stoichiometry for the $1 . \mathrm{Py}_{2} \mathrm{C}_{60}$ complex.

The complex appears to be stable even at $50{ }^{\circ} \mathrm{C}$. Subsequent addition of one equivalent of $\mathrm{F}^{-}$to the solution of $1 . \mathrm{Py}_{2} \mathrm{C}_{60}$ resulted in the disappearance of the broad peak due to the $\mathrm{NH}$ protons of OXP (see Figure 5.2.3b) indicating $\mathrm{F}^{-}$binding at the OXP anion binding site. The fullerene complexation appears not to be affected even in the presence of up to four equivalents of fluoride anions. These results, along with the earlier discussed optical absorption studies, prove the structural integrity of the supramolecular complex depicted in scheme 5.2.1.

\subsubsection{Electrochemical Studies}

Cyclic voltammetry studies were performed to evaluate the redox potentials of the individual entities and that of the supramolecular complex to estimate the freeenergy change associated with the photo-induced electron transfer events. As reported earlier, the OXP and its $\mathrm{N}$-substituted derivatives possess extended $\pi$-conjugation with four hemiquinone substituents at its periphery, so that it is expected to undergo facile oxidation and reduction processes. ${ }^{191,242}$ Furthermore, the redox-active N-substituents are expected to influence the redox potentials of the OXP core in addition to exhibiting their own redox processes. As shown in Figure 5.2.4b (i) the first oxidation and first reduction processes of $\operatorname{OXP}(\mathrm{bz})_{2}$ are located at 0.48 and $-1.37 \mathrm{~V}$, respectively, vs 
$\mathrm{Fc} / \mathrm{Fc}^{+}$. Replacing the $\mathrm{N}$-benzyl substituents with zinc porphyrin entities leads to the appearance of additional redox processes corresponding to the $\mathrm{ZnP}$ entities (Figure $5.2 \cdot 4 a)$.

(a)

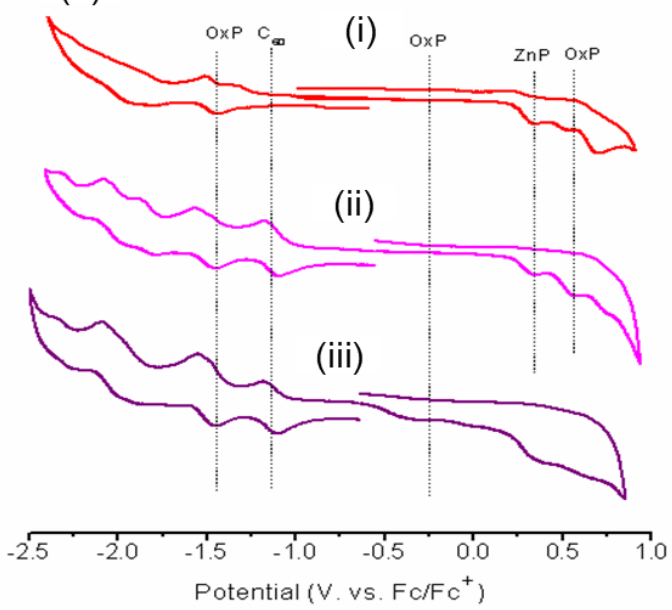

Potential (V.vs. Fc/FC ${ }^{+}$)

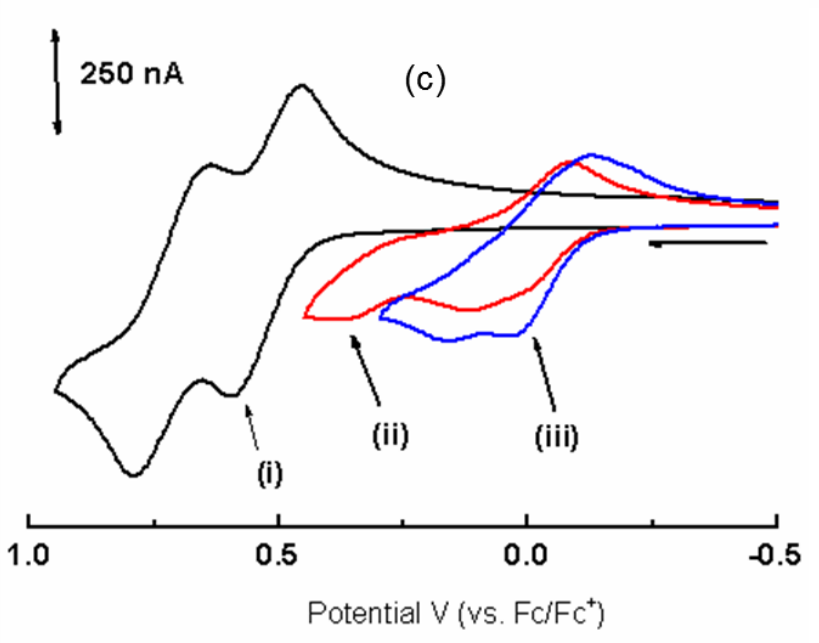

(b)
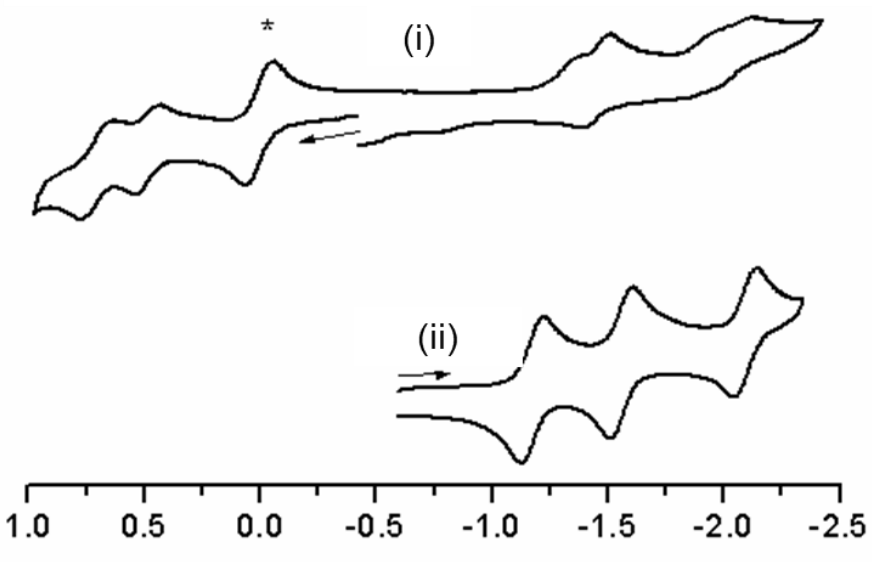

Potential V (vs. $\left.\mathrm{Fc} / \mathrm{Fc}^{+}\right)$

Figure 5.2.4: a) Cyclic voltammograms of (i) 1, (ii) 1. $\mathrm{Py}_{2} \mathrm{C}_{60}$ obtained by equimolar mixture of 1 and $\mathrm{Py}_{2} \mathrm{C}_{60}$, and (iii) 1(F-). $\mathrm{Py}_{2} \mathrm{C}_{60}$ obtained by equimolar mixture of 1 . $\mathrm{Py}_{2} \mathrm{C}_{60}$ and $\mathrm{F}^{-}$. b) Cyclic voltammograms of (i) OXP-(bz) $)_{2}$ and (ii) $\mathrm{Py}_{2} \mathrm{C}_{60}$ in Ar-saturated o-dichlorobenzene containing $0.1 \mathrm{M}\left(\mathrm{n}-\mathrm{Bu}_{4} \mathrm{~N}\right) \mathrm{ClO}_{4}$. Scan rate $=0.1 \mathrm{~V} / \mathrm{s}$. The asterisk $\left.{ }^{*}\right)$ represents ferrocene redox couple added as an internal standard. c) Cyclic Voltammograms of $\mathrm{OXP}(\mathrm{Bz})_{2}$ on (i) 0 eq. (ii) 1.0 eq. and (iii) 2.0 eq. addition of ( $\mathrm{n}$ $\left.\mathrm{Bu}_{4} \mathrm{~N}\right) \mathrm{F}^{-}$and in o-dichlorobenzene containing $0.1 \mathrm{M}\left(\mathrm{n}-\mathrm{Bu}_{4} \mathrm{~N}\right) \mathrm{PF}_{6}$. Scan rate $=100$ $\mathrm{mV} / \mathrm{s}$. All measurements performed in o-dichlorobenzene containing $0.1 \mathrm{M}\left(\mathrm{n}-\mathrm{Bu}_{4} \mathrm{~N}\right) \mathrm{PF}_{6}$. 
The first oxidation involving $\mathrm{ZnP}$ is located at $0.28 \mathrm{~V}$ while the first reduction involving the electron deficient OXP macrocycle is located at $-1.37 \mathrm{~V}$ vs $\mathrm{Fc} / \mathrm{Fc}^{+}$.

The first three reductions of fullerene, $\mathrm{Py}_{2} \mathrm{C}_{60}$, are located at $-1.12,-1.50$ and $-2.04 \mathrm{~V}$ vs. $\mathrm{Fc} / \mathrm{Fc}^{+}$(see Figure 5.2.4 (ii)). Upon forming $1 . \mathrm{Py}_{2} \mathrm{C}_{60}$ by equimolar addition of 1 to the solution of $\mathrm{Py}_{2} \mathrm{C}_{60}$ exhibited additional reduction processes corresponding to the fulleropyrrolidine entity (see Figure 5.2.4 (ii)). The reduction potentials of the fullerene and $\mathrm{ZnP}$ entities revealed a small $(\sim 20 \mathrm{mV})$ potential shift upon axial coordination. The electrochemically evaluated HOMO (ZnP)-LUMO $\left(\mathrm{py}_{2} \mathrm{C}_{60}\right)$ gap for 1. $\mathrm{Py}_{2} \mathrm{C}_{60}$ was found to be $\sim 1.40 \mathrm{~V}$.

Anion binding to OXP is expected to exhibit large cathodic shifts of the oxidation potentials since the binding site is contained within the extended $\pi$-conjugated system. For example, $\mathrm{F}^{-}$binding to $\mathrm{OXP}(\mathrm{bz})_{2}$ exhibited an cathodic shift of nearly $600 \mathrm{mV}$ for the first oxidation process ${ }^{189}$ (see Figure 5.2.4a(iii)). Similar potential shifts were observed for other strongly binding anions; however, weakly binding anions such as $\mathrm{ClO}_{4}{ }^{-}$caused no substantial potential shifts. ${ }^{189}$ This seems also to be the case when strongly interacting anions bind to the 1.Py $\mathrm{C}_{60}$ complex, as shown in Figure 5.2.4a (iii). The OXP oxidation peak of the $1 . \mathrm{Py}_{2} \mathrm{C}_{60}$ complex located at $0.48 \mathrm{~V}$, although irreversible, anodically shifted by over $600 \mathrm{mV}$ upon binding to $\mathrm{F}^{-}$. It is important to note that in the presence of $\mathrm{F}^{-}$, both the redox potentials of both $\mathrm{ZnP}$ and $\mathrm{C}_{60}$ did not show significant changes, once again indicating no direct interaction of these entities with the anions. Similar voltammetric behavior was observed for strongly interacting acetate and dihydrogen phosphate anions but not for the weakly interacting perchlorate anions. The easier oxidation of strongly interacting anion bound OXP compared to $\mathrm{ZnP}$ suggests it 
to act as a terminal electron donor in the supramolecular complex, $1\left(\mathrm{~F}^{-}\right) \cdot \mathrm{Py}_{2} \mathrm{C}_{60}$ affording the final CS state via photoinduced electron transfer from singlet excited porphyrinatozinc $\left.(1 \mathrm{ZnP})^{*}\right)$ to the coordinated fullerene electron acceptor $\left(\mathrm{Py}_{2} \mathrm{C}_{60}\right)$, followed by electron transfer from $\mathbf{1}\left(\mathrm{F}^{-}\right)$to $\mathrm{ZnP} \cdot+$.

\subsubsection{Steady-State Fluorescence Studies}

The $\mathrm{ZnP}$ utilized in the construction of 1 emits at 613 and $667 \mathrm{~nm}$ while the OXP entity shows a broad emission with a peak maxima at $724 \mathrm{~nm}$, respectively. Owing to the presence of electron deficient OXP, the emission bands of $\mathbf{1}$ are quenched substantially as shown in Figure 5.2.5a. ${ }^{187} \mathrm{The} \mathrm{ZnP}$ emission bands appear as shoulder bands to the OXP emission bands. The fluorescence spectrum of 1 revealed additional quenching
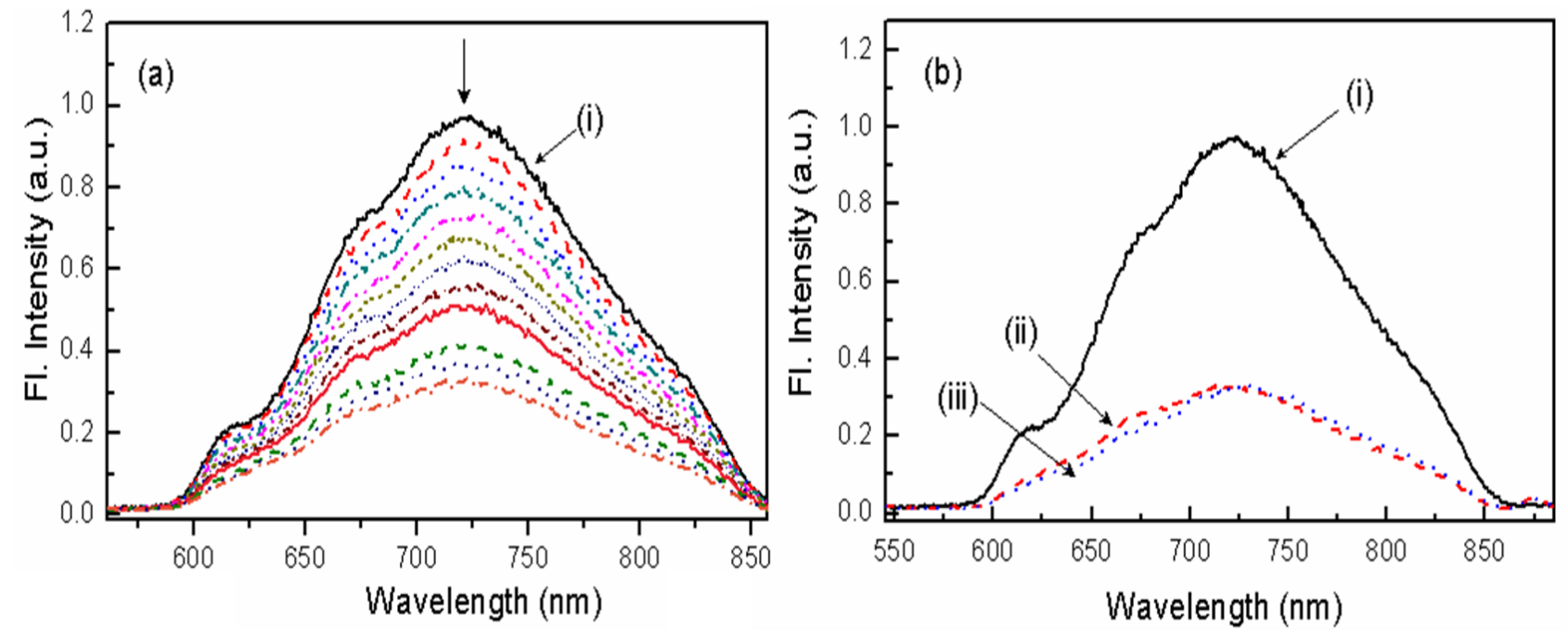

Figure 5.2.5: Steady-state fluorescence spectrum of (a) 1 in on increasing addition of $\mathrm{Py}_{2} \mathrm{C}_{60}$ to form the 1. $\mathrm{Py}_{2} \mathrm{C}_{60}$ complex (1.1 eq total), and (b) on addition of $\mathrm{F}^{-}$(1.1 eq. total) to the solution of $1 \mathrm{Py}_{2} \mathrm{C}_{60}$ to form the $\mathbf{1}(\mathrm{F}-)$. $\mathrm{Py}_{2} \mathrm{C}_{60}$ complex in o-dichlorobenzene. The samples were excited at $440 \mathrm{~nm}$ corresponding to $\mathrm{ZnP}$ Soret band and the initial concentration of 1 was held at $50 \mu \mathrm{M}$. Spectrum (i) represents that of 1 in the absence of guest entities, (ii) represents that of 1 . $\mathrm{Py}_{2} \mathrm{C}_{60}$ complex, and (iii) represents that of 1( $\left.\mathrm{F}^{-}\right) . \mathrm{Py}_{2} \mathrm{C}_{60}$ complex. 
upon addition of $\mathrm{Py}_{2} \mathrm{C}_{60}$ to form 1.Py $\mathrm{C}_{60}$ as shown in Figure 5.2.5a. Interestingly, addition of $\mathrm{F}^{-}$to the $1 . \mathrm{Py}_{2} \mathrm{C}_{60}$ solution had little or no changes in the peaks corresponding to both $\mathrm{ZnP}$ and $\mathrm{OXP}$ entities as shown in Figure 5.2.5b. Based on energy calculations, quenching in $1 . \mathrm{Py}_{2} \mathrm{C}_{60}$ could be attributed to photoinduced electron transfer from singlet excited $\mathrm{ZnP}$ to coordinated fullerene (vide infra). Further, timeresolved emission and transient absorption spectral studies were performed to unravel the quenching mechanism and effect of $\mathrm{F}^{-}$on kinetics of electron transfer in the supramolecular complex.

\subsubsection{Transient Studies}

Femtosecond Laser Flash Photolysis of the Supramolecular Complex between 1 and $\mathrm{Py}_{2} \mathrm{C}_{60}$

Femtosecond transient absorption spectroscopy of the supramolecular complex $\left(\mathbf{1}\left(\mathrm{F}^{-}\right) \cdot \mathrm{Py}_{2} \mathrm{C}_{60}\right)$ with excitation at $440 \mathrm{~nm}$ reveals the transient absorption band at $900 \mathrm{~nm}$ due to the singlet excited state of the $\mathrm{ZnP}$ moiety of the supramolecular complex with the bleaching of the $\mathrm{Q}$ band of the $\mathrm{ZnP}$ moiety at $600 \mathrm{~nm}$ as shown in Figure 5.2.6a. To our surprise the transient absorption disappears completely at $100 \mathrm{ps}$ and the lifetime of the singlet excited state is determined to be 34 ps by the single exponential curve fitting in Figure 5.2.6b No triplet absorption due to $\mathrm{ZnP}$ or $\mathrm{Py}_{2} \mathrm{C}_{60}$ was observed. Under the experimental conditions in Figure 5.2.6 the concentration of $1, \mathrm{Py}_{2} \mathrm{C}_{60}$ and $\mathrm{F}^{-}$is $1.5 \times 10^{-}$

${ }^{4} \mathrm{M}$ when $97 \%$ of each component forms the supramolecular complex judging from the $K$ value in Table 5.2.1 $\left(1.9 \times 10^{5} \mathrm{M}^{-1}\right)$. This is consistent with the fast decay of the singlet excited state of the $\mathrm{ZnP}$ moiety $\left({ }^{1} \mathrm{ZnP}{ }^{*}\right)$ in the supramolecular complex because there would be the long-lived component of the singlet excited state of the $\mathrm{ZnP}$ moiety of free 
1 molecules $(\tau=1.9 \mathrm{~ns} \text { for } \mathrm{ZnP})^{187}$ if free 1 molecules remained in solution were excited. The fast decay of ${ }^{1} \mathrm{ZnP}{ }^{*}$ may result from the fast electron transfer from ${ }^{1} \mathrm{ZnP}{ }^{*}$ to $\mathrm{Py}_{2} \mathrm{C}_{60}$ in the supramolecular complex.

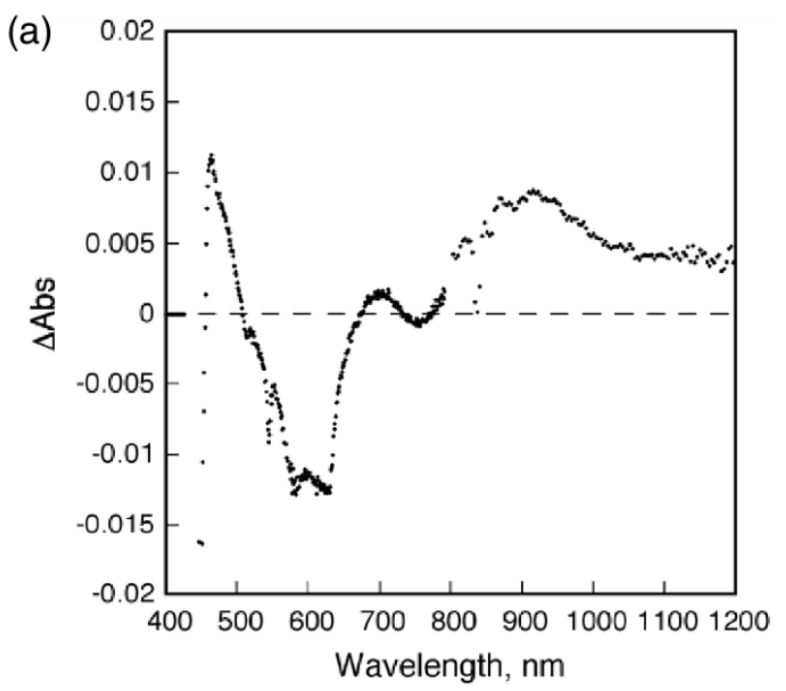

(b)

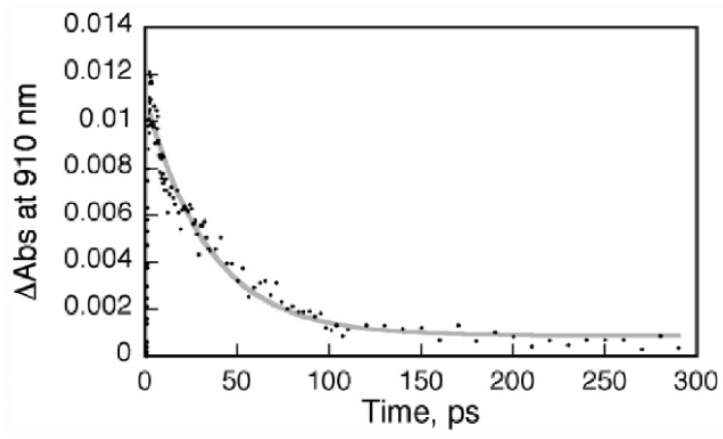

Figure 5.2.6: (a) Transient absorption spectrum of $\mathbf{1}\left(\mathrm{F}^{-}\right)$. $\mathrm{Py}_{2} \mathrm{C}_{60}$ in o-dichlorobenzene (concentration of each component: $1.5 \times 10^{-4} \mathrm{M}$ ) at $2 \mathrm{ps}$ after femtosecond laser excitation at $440 \mathrm{~nm}$. (b) Decay of absorbance at $910 \mathrm{~nm}$ corresponding to the singlet excited state of the $\mathrm{ZnP}$ moiety in the supramolecular complex

The absence of the absorption at $1000 \mathrm{~nm}$ due to $\mathrm{Py}_{2} \mathrm{C}_{60}{ }^{-}{ }^{-}$in the transient absorption spectra suggests that back electron transfer from $\mathrm{Py}_{2} \mathrm{C}_{60}{ }^{\cdot-}$ to $\mathrm{ZnP}{ }^{\cdot+}$ to the ground state is much faster than the forward electron transfer. In such a case, the rate constant of electron transfer from ${ }^{1} \mathrm{ZnP}{ }^{*}$ to $\mathrm{Py}_{2} \mathrm{C}_{60}$ in the supramolecular complex to produce the $\mathrm{CS}$ state $\left(k_{\mathrm{CS}}\right)$ is determined to be $2.9 \times 10^{10} \mathrm{~s}^{-1}$ from the single exponential curve fitting in Figure 5.2.6b. Similar results were obtained for the supramolecular complexes with binding of different anions. Figure 5.2.7a shows the transient absorption spectra of the supramolecular complex with binding of acetate ion. The $k_{\mathrm{CS}}$ value is determined to be $1.2 \times 10^{10} \mathrm{~s}^{-1}$ from the single exponential curve fitting in Figure 5.2.7b. This value is smaller than the value obtained for $\mathrm{F}^{-}$binding. The $k_{\mathrm{CS}}$ values determined for other 
anions are listed in Table 5.2.1. The $k_{\mathrm{CS}}$ value decreases in order: $2.9 \times 10^{10}\left(\mathrm{~F}^{-}\right)>1.5 \mathrm{x}$ $10^{10}\left(\mathrm{H}_{2} \mathrm{PO}_{4}^{-}\right)>1.2 \times 10^{10}\left(\mathrm{CH}_{3} \mathrm{COO}^{-}\right)>1.2 \times 10^{9}\left(\mathrm{ClO}_{4}^{-}\right)>6.3 \times 10^{8}$ (none) $\mathrm{s}^{-1}$. Generally, the stronger is the binding, the faster becomes the electron transfer rates.

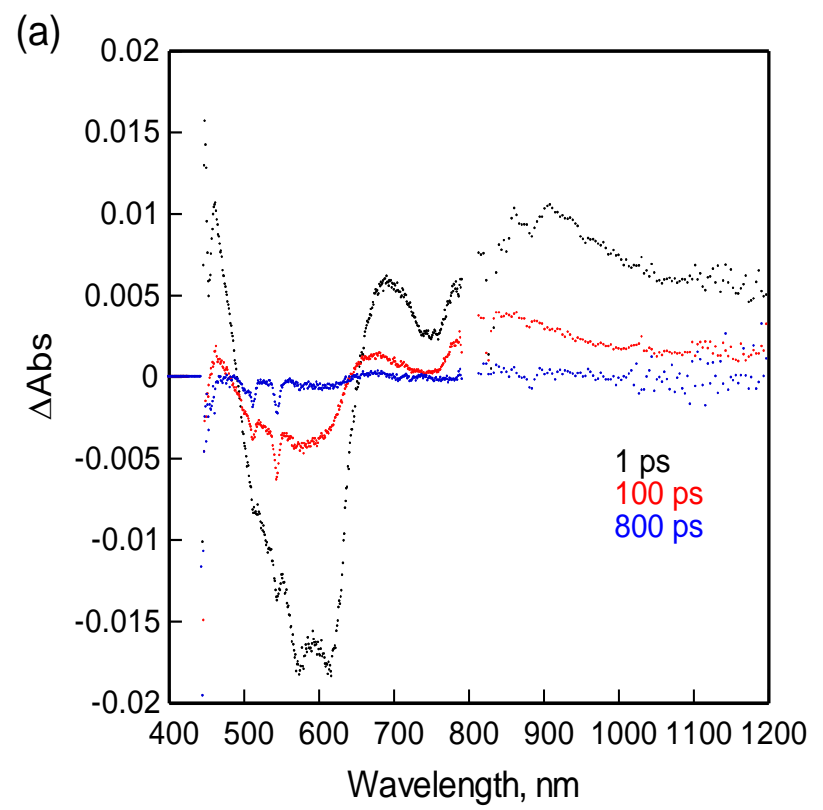

(b)

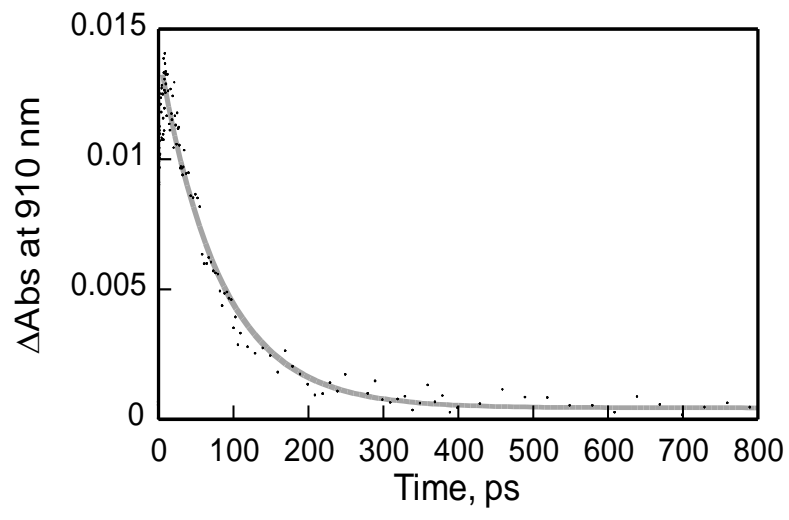

Figure 5.2.7: (a) Transient absorption spectrum of $1\left(\mathrm{CH}_{3} \mathrm{COO}^{-}\right)$. $\mathrm{Py}_{2} \mathrm{C}_{60}$ in 0 dichlorobenzene (concentration of each component: $1.5 \times 10-4 \mathrm{M}$ ) at 2 ps (black), 500 ps (red), 3000 ps (blue) after femtosecond laser excitation at $440 \mathrm{~nm}$. (b) Decay of absorbance at $910 \mathrm{~nm}$ corresponding to the singlet excited state of the ZnP moiety in the supramolecular complex.

The CS State of the Supramolecular Complex between 1 and $\mathrm{Py}_{2} \mathrm{C}_{60}$ detected by Nanosecond Laser Flash Photolysis.

In contrast to the case of femtosecond laser flash photolysis in Figure 5.2.8a, the nanosecond transient absorption spectroscopy of $\mathbf{1}\left(\mathrm{F}^{-}\right) \cdot \mathrm{Py}_{2} \mathrm{C}_{60}$ with excitation at $450 \mathrm{~nm}$ (Figure 5.2.8a) revealed the absorption band due to the fulleropyrrolidine anion radical at $1000 \mathrm{~nm}$ together with the broad absorption in the region of $700-800 \mathrm{~nm}$. The absorption band at $700 \mathrm{~nm}$ is assigned to the triplet excited state of $\mathrm{Py}_{2} \mathrm{C}_{60}{ }^{15}$ The additional broad absorption band may be assigned to the radical cation of $\mathbf{1}\left(\mathrm{F}^{-}\right)$. $^{191,242}$ 
This indicates the presence of a CS species while kinetic measurements performed by monitoring the decay of the fullerene anion radical at $1000 \mathrm{~nm}$ giving a surprisingly long lifetime for this species $(14 \mu \mathrm{s})$ as shown in Figure 5.2.8b.

Since the singlet excited state $\left({ }^{1} \mathrm{ZnP} \mathrm{P}^{*}\right)$ in the supramolecular complex decays completely at $100 \mathrm{ps}$ as shown in Figure 5.2.6b, the triplet excited state $\left({ }^{3} \mathrm{ZnP}{ }^{*}\right)$ rather than the singlet excited state $\left({ }^{1} \mathrm{ZnP}^{*}\right)$ should be involved for the formation of the CS state. Under the experimental conditions in Figure 5.2.8, concentration of each component $\left(1, \mathrm{Py}_{2} \mathrm{C}_{60}\right.$ and $\left.\mathrm{F}^{-}\right)$is $3.0 \times 10^{-5} \mathrm{M}$, which is smaller than that employed for the femtosecond laser flash photolysis, because a $1 \mathrm{~cm}$ path length cell was used for the nanosecond laser flash photolysis, whereas a $2 \mathrm{~mm}$ cell was used for the femtosecond laser flash photolysis). In such a case $15 \%$ molecules of each component remain as uncomplexed and the $\mathrm{ZnP}$ moiety of the free molecules was excited with nanosecond laser excitation at $450 \mathrm{~nm}$ to produce the triplet excited state $\left({ }^{3} \mathrm{ZnP}^{*}\right)$ via intersystem crossing from ${ }^{1} \mathrm{ZnP}^{*}$ at the microsecond time scale in Figure 5.2.8. Then, intermolecular electron transfer from ${ }^{3} \mathrm{ZnP}^{*}$ to $\mathrm{Py}_{2} \mathrm{C}_{60}$ may occur to afford the triplet supramolecular CS state. The dynamics of electron transfer from ${ }^{3} \mathrm{ZnP}^{*}$ to $\mathrm{Py}_{2} \mathrm{C}_{60}$ is too fast to be monitored by nanosecond laser flash photolysis (inset of Figure 5.2.8b). On the other hand, the process of electron transfer from $\mathrm{ZnP}$ to lifetime of ${ }^{3} \mathrm{Py}_{2} \mathrm{C}_{60}{ }^{*}$ could be observed as shown in the inset of Figure 5.2.8b. The ${ }^{3} \mathrm{C}_{60}{ }^{\star}$ is generated via energy transfer from ${ }^{1} \mathrm{ZnP}^{*}$ to $\mathrm{C}_{60}$ and the intersystem crossing. The intermolecular second-order rate constant of electron transfer from $\mathrm{ZnP}$ to ${ }^{3} \mathrm{C}_{60}{ }^{*}$ was determined to be $1.5 \times 10^{10} \mathrm{M}^{-1} \mathrm{~s}^{-1}$ from the rise of absorbance at $1000 \mathrm{~nm}$ due to $\mathrm{C}_{60}{ }^{-}$. The single exponential decay of the CS state (Figure 5.2.8b) indicates the formation of 
the supramolecular complex following the intermolecular photoinduced electron-transfer reaction. When the concentration of $1\left(\mathrm{~F}^{-}\right) \cdot \mathrm{Py}_{2} \mathrm{C}_{60}$ was decreased, the quantum yield of CS state $[\Phi(\mathrm{cs})]$, determined from comparative method, ${ }^{243}$ increased to 0.14 at $3.0 \times 10^{-}$ ${ }^{5} \mathrm{M}$ of equimolar $\mathbf{1}\left(\mathrm{F}^{-}\right)$and $\mathrm{Py}_{2} \mathrm{C}_{60}$ as shown in Figure 5.2.9.

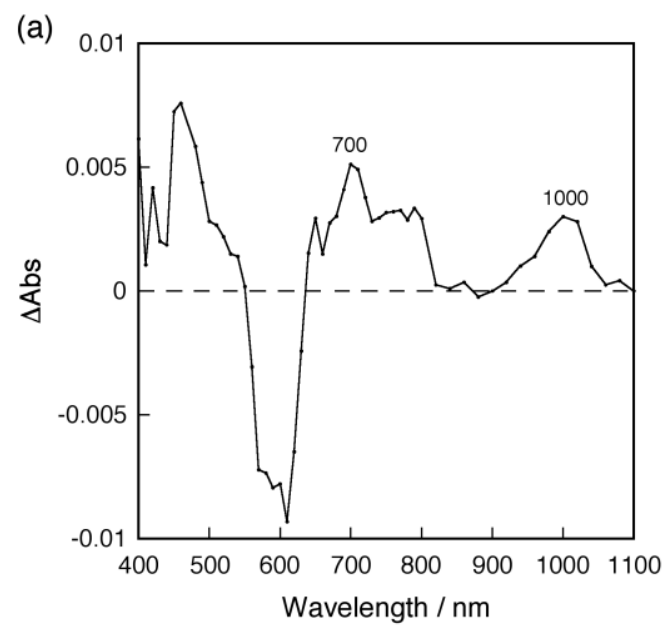

(b)
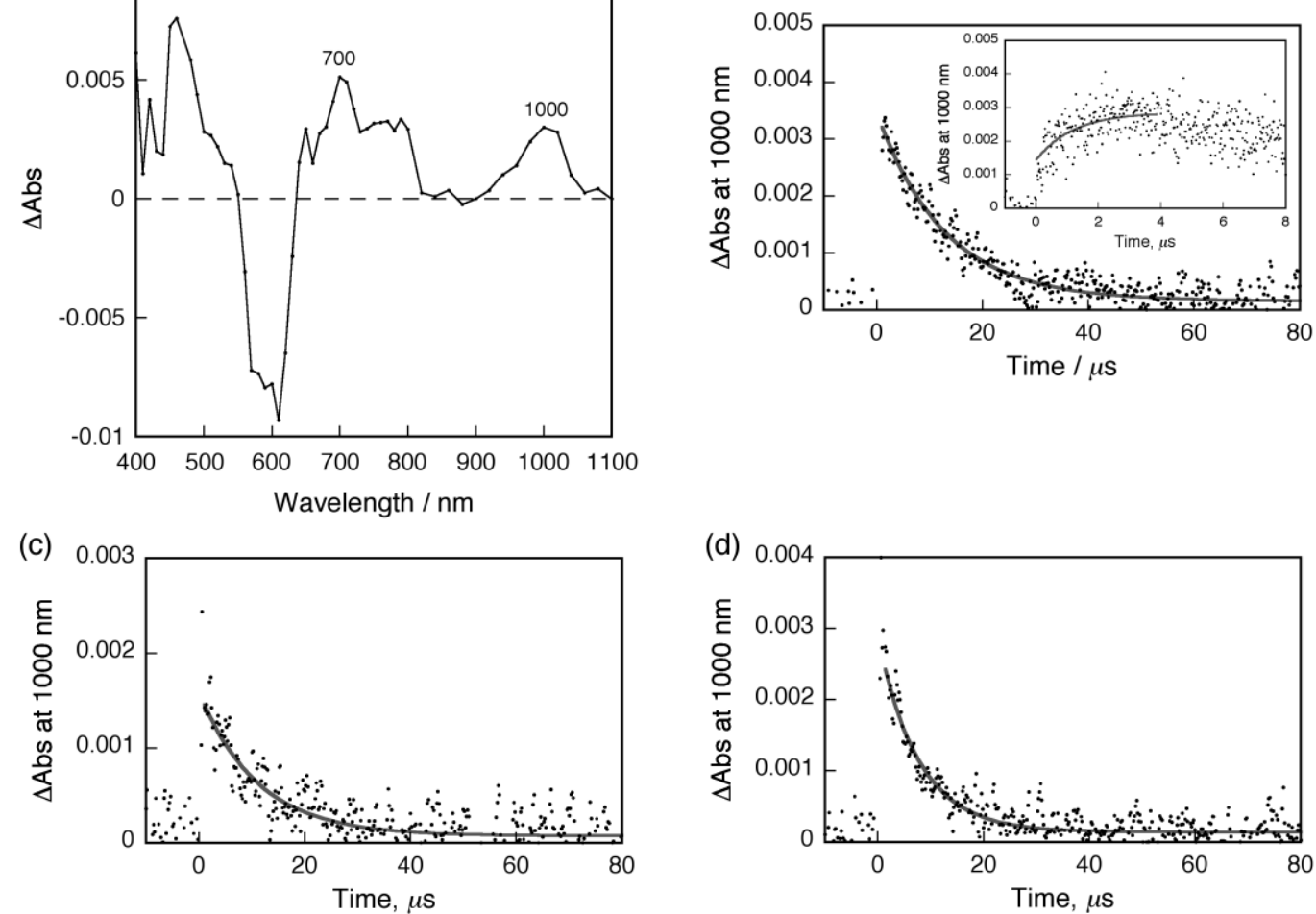

Figure 5.2.8: (a) Transient absorption spectrum of $1\left(\mathrm{~F}^{-}\right) \cdot \mathrm{Py}_{2} \mathrm{C}_{60}$ in o-dichlorobenzene (concentration of each component: $3.0 \times 10^{-5} \mathrm{M}$ ) at $1 \mu \mathrm{s}$ after nanosecond laser excitation at $450 \mathrm{~nm}$. (b) Decay of absorbance for $\mathbf{1}\left(\mathrm{F}^{-}\right) \cdot \mathrm{Py}_{2} \mathrm{C}_{60}$ at $1000 \mathrm{~nm}$ corresponding to fullerene anion radical. Inset: 0-8 $\mu$ s time range (c) Decay of absorbance for $\mathbf{1}\left(\mathrm{CH}_{3} \mathrm{COO}^{-}\right) \cdot \mathrm{Py}_{2} \mathrm{C}_{60}$ at $1000 \mathrm{~nm}$. (d) Decay of absorbance for $\mathbf{1}\left(\mathrm{H}_{2} \mathrm{PO}_{4}\right) \cdot \mathrm{Py}_{2} \mathrm{C}_{60}$ at $1000 \mathrm{~nm}$.

The observed quantum yields depending on concentration of equimolar $\mathbf{1}\left(\mathrm{F}^{-}\right)$and $\mathrm{Py}_{2} \mathrm{C}_{60}$ agreed with the ratio of the free porphyrin component determined from the formation constant of the supramolecular complex $\left(K_{a}=1.9 \times 10^{5} \mathrm{M}^{-1}\right.$ in Table 5.2.1). The observed long lifetime of the CS state may be a consequence of the persistence of 
the triplet-correlated radical-ion pair character in the CS state, which results in the corresponding forbidden charge recombination back to the singlet ground state in contrast to the case of the singlet CS state in Figure 5.2.6. The triplet multiplicity of the CS state, which is populated from the intermolecular electron transfer from ${ }^{3} \mathrm{ZnP}$ to $\mathrm{Py}_{2} \mathrm{C}_{60}$ or from $\mathrm{ZnP}$ to ${ }^{3} \mathrm{Py}_{2} \mathrm{C}_{60}{ }^{*}$, plays a crucial role in determining its very long lifetime of $14 \mu \mathrm{s}$. The spin conversion from the triplet supramolecular CS state to the singlet CS state may be prohibited because of the short distance between the radical ions in the supramolecular complex, which results in a large magnitude of the spin exchange interaction (J). ${ }^{244-247}$ Additionally, we have examined nanosecond laser flash experiments in the presence of $\mathrm{O}_{2}$ to estimate the spin state of the CS state. No transient absorption due to the CS state of $\mathbf{1}(\mathrm{F}-)$. $\mathrm{Py}_{2} \mathrm{C}_{60}$ was observed in aerated odichlorobenzene. The CS state was efficiently quenched by $\mathrm{O}_{2}$ via energy transfer since electron transfer from $\mathrm{C}^{\circ} 0^{\circ-}$ of the CS state would be relatively slow. ${ }^{248}$ This confirms that the long-lived CS state is a triplet radical ion pair. The triplet supramolecular CS lifetime decreases with weaker binding of anions as indicated by the decay time profile of absorbance at $1000 \mathrm{~nm}$ for $\mathbf{1}\left(\mathrm{CH}_{3} \mathrm{COO}^{-}\right) \cdot \mathrm{Py}_{2} \mathrm{C}_{60}(11 \mu \mathrm{s}), \mathbf{1}\left(\mathrm{H}_{2} \mathrm{PO}_{4}{ }^{-}\right.$ ). $\mathrm{Py}_{2} \mathrm{C}_{60}(7.5 \mu \mathrm{s})$ in Figure $5.2 .8 \mathrm{c}$ and $5.2 .8 \mathrm{~d}$, respectively. In the case of $\mathrm{ClO}_{4}{ }^{-}$, no anion binding was observed when the CS lifetime $(0.17 \mu \mathrm{s})$ is the same as that without anion $(0.16 \mu \mathrm{s})$. Thus, the introduction of the fluoride cofactor into the $1 . \mathrm{Py}_{2} \mathrm{C}_{60}$ complex results in an around 90-fold enhancement of the lifetime of the CS species over the noncomplexed 1.Py $\mathrm{C}_{60}$.

In the absence of $\mathrm{F}^{-}$, excitation of the porphyrinatozinc results primarily in charge separation leading to the formation of $\mathrm{ZnP}^{++}-\mathrm{C}_{60}{ }^{--}$(energy $=1.40 \mathrm{eV}$ ) that persists for a 
few hundred nanoseconds before relaxing to the ground state. Complexation of $\mathrm{F}^{-}$to the OXP center lowers its oxidation potential by nearly $600 \mathrm{mV}^{191,242}$ (see Figure 5.2.4) thus creating an intermediate energy state for charge migration from the $\mathrm{ZnP}^{+\cdot}$ to the $\mathrm{F}^{-}$ :OXP center (energy $=<0.83 \mathrm{eV}$ ). The increase in the reorganization energy of electron transfer combined with the decrease in $\mathrm{CR}$ driving force caused by $\mathrm{F}^{-}$binding results in a reduction in the rate of the CR process and an increase in the lifetime of the triplet supramolecular CS state.

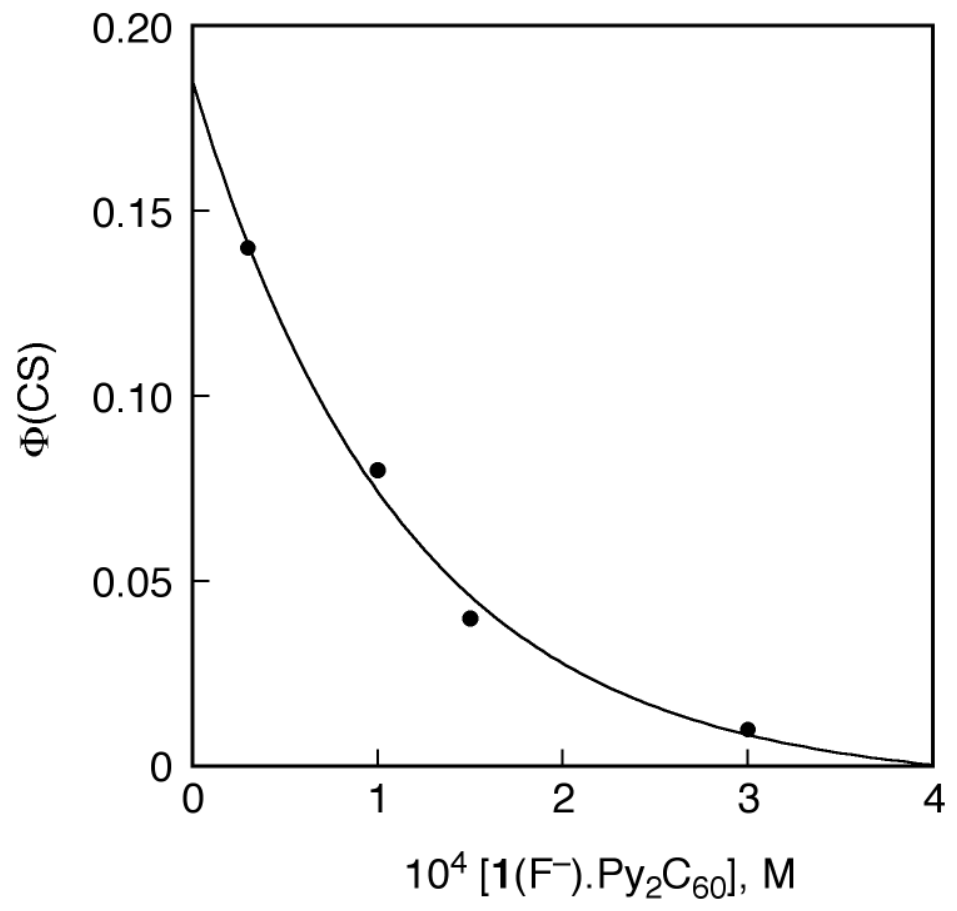

Figure 5.2.9: Plot of the quantum yield of the CS state of $1\left(\mathrm{~F}^{-}\right) \cdot \mathrm{Py}_{2} \mathrm{C}_{60}[\Phi(\mathrm{CS})]$ vs concentration of equimolar $\mathbf{1}\left(\mathrm{F}^{-}\right)$and $\mathrm{Py}_{2} \mathrm{C} 60$ in o-dichlorobenzene

\subsubsection{Summary}

We have observed the first example of anion-complexation-induced enhancement of the charge separation in a supramolecular complex to produce the singlet CS states and also stabilization of the triplet CS states in an oligochromophoric molecule possessing exclusive binding sites for both a guest electron acceptor and an 
anionic cofactor species. This work not only illustrates a novel method for controlling persistence of CS states but also demonstrates the great utility of the supramolecular assembly route to complex functional systems. Finally, it is important to note the indispensability of the oxoporphyrinogen framework in this system since it allows for the selective positioning of substituents by virtue of its special reactivity towards alkylating reagents and permits electrochemical control of its redox potentials in the presence of inorganic anions.

\subsection{Porphyrin-Oxoporphyrinogen Surface Modified $\mathrm{TiO}_{2}$ Supramolecular Solar Cell ${ }^{\star *}$}

\subsubsection{Introduction}

Events triggered by anion binding to redox- or photo-active synthetic host molecules have been put to a variety of applications in recent years especially in the area of anion recognition and sensing. ${ }^{249-254}$ Extensive research effort has been made to understand how the host structures undergo physico-chemical changes upon anion binding with an ultimate goal of discovering applications beyond sensing, in particular for catalysis, separation, and energy harvesting. ${ }^{249-254}$

Stabilization of charge-separated states in donor-acceptor molecules in the form of long-lived radical ion-pair species is crucial for these molecules to be useful for photocatalytic and solar energy harvesting applications. ${ }^{11,47,92,152,156,179,181}$ Anion binding to a redox active receptor could facilitate such a process by modulating the redox potentials of the donor and/or acceptor entities thereby altering the free-energy change associated with electron-transfer events. Recently, we demonstrated photochemical

\footnotetext{
** Section 5.3 in its entirety is reproduced from the Chemical Communication, 2011, 47, pp 6003- 6005 with permission from the publisher.
} 
charge stabilization in an oxoporphyrinogen-bisfullerene dyad ${ }^{255}$ and in an oxoporphyrinogen-bis-zinc porphyrin-fullerene triad upon $\mathrm{F}^{-}$binding to the redox-active OXP anion binding site. ${ }^{186}$ In these supramolecular systems, $\mathrm{F}^{-}$binding shifts cathodically the oxidation potential of OXP by as much as $500 \mathrm{mV}^{189}$ thereby facilitating electron transfer (in the dyad) or hole transfer (in the triad), resulting in generation of charge stabilized states. However, the effect of anion binding induced charge stabilization on the performance of light energy harvesting devices has not been evaluated. In the present study, we have verified the benefit of the latter by constructing a novel supramolecular solar cell.

Scheme 5.3.1 depicts the design of the supramolecular solar cell constructed for this purpose based on biomimetic principles. ${ }^{167}$ The sensitizing ZnP-OXP dyad was immobilized by metal-ligand axial coordination onto a thin-film semiconducting $\mathrm{TiO}_{2}$ surface functionalized with phenylimidazole coordinating ligand on an FTO transparent electrode. The $\mathrm{ZnP}$ entity of the dyad is coordinated to the imidazole ligand leaving the OXP site available for anion binding. The $\mathrm{ZnP}-\mathrm{OXP}: \mathrm{F}^{-}$species formed upon $\mathrm{F}^{-}$binding revealed a cathodic shift of the OXP: $\mathrm{F}^{-}$oxidation by about $450 \mathrm{mV}$, that is, about 380 $\mathrm{mV}$ easier than the first oxidation potential of $\mathrm{ZnP}$. The easier oxidation of OXP: $\mathrm{F}^{-}$ compared to the primary electron donor, $\mathrm{ZnP}$ makes the former a hole- transfer agent to $\mathrm{ZnP}^{+.}$during electron-transfer events thus slowing down the back electron transfer from the one-electron reduced $\mathrm{TiO}_{2}$ to the $\mathrm{ZnP}^{++} \cdot{ }^{167}$ As demonstrated here, consequently, this should improve the overall photo-conversion efficiency of the photocell. ZnP-OXP was synthesized by a reported method. ${ }^{256,257}$ This involved initial synthesis of bromomethyl-substituted tetraphenylporphyrin and its subsequent use in $\mathrm{N}$-alkylation of 
oxoporphyrinogen. Finally, the free-base porphyrin was metallated using zinc acetate followed by chromatographic purification to obtain the desired ZnP-OXP dyad.

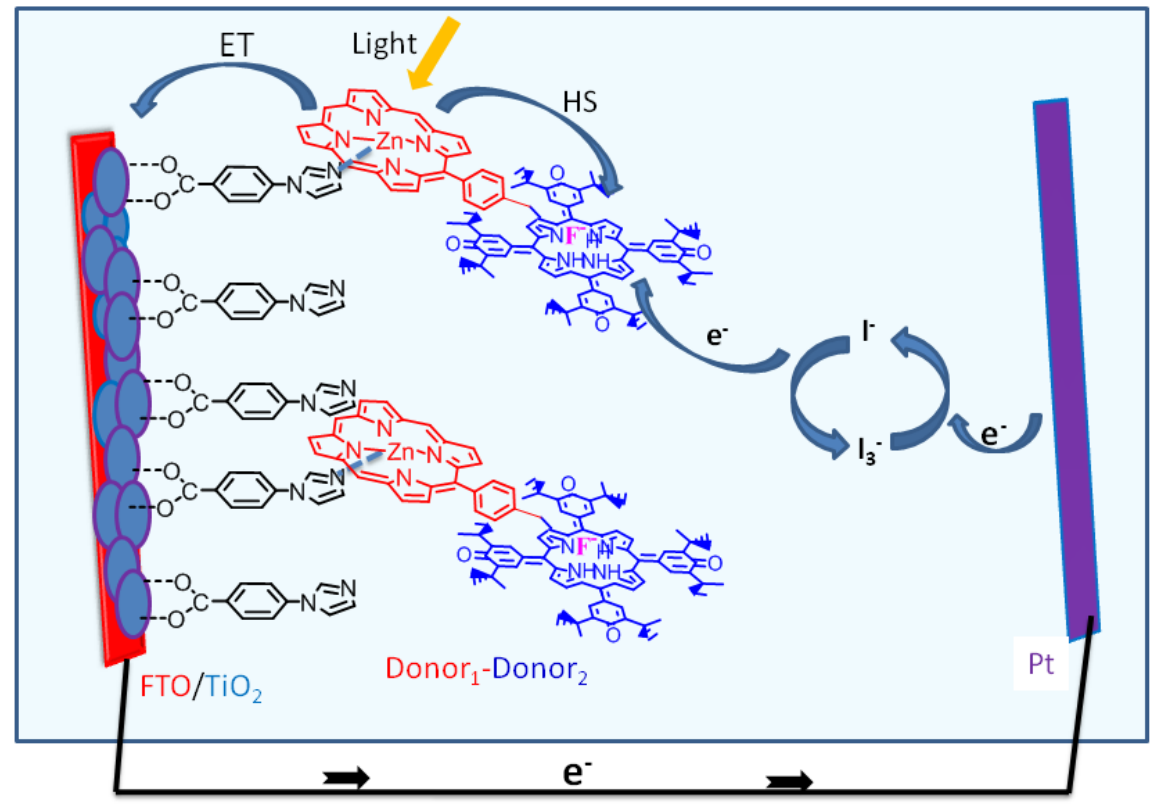

Scheme 5.3.1: Schematic figure of the zinc porphyrin-oxoporphyrinogen (ZnP-OXP) surface modification using axial coordination onto the $\mathrm{TiO}_{2} / \mathrm{FTO}$ solar cell to demonstrate enhanced photocurrent generation upon $\mathrm{F}^{-}$binding to OXP. The photochemical electron- and hole- transfer processes are shown by the arrows

\subsubsection{Results and Discussion}

\subsubsection{Optical Studies}

The preferential metal-ligand binding through the $\mathrm{ZnP}$ entity, and $\mathrm{F}^{-}$binding to the OXP entity of the dyad were established from optical absorption studies involving additional control compounds. The dyad ZnP-OXP exhibits bands at 423, 549 and 588 $\mathrm{nm}$ corresponding to the $\mathrm{ZnP}$ entity while the OXP entity leads to a relatively broad band at $515 \mathrm{~nm}$. Comparison of the spectrum with those of control compounds, zinc tetraphenylporphyrin (ZnTPP) and oxoporphyrinogen revealed no changes in the peak positions (Figure. 5.3.1) suggesting little or no electronic interactions between the entities of the covalently linked dyad. 




Figure 5.3.1: Normalized absorption spectra of (i) ZnP-OXP dyad, (ii) ZnTPP and (iii) OXP in DCB.

Figure 5.3.2a shows phenylimidazole binding to $\mathrm{ZnP}$-OXP in o-dichlorobenzene (DCB). Addition of phenylimidazole resulted in red shifts of the absorption bands of ZnP with isosbestic points at 428,447 and $567 \mathrm{~nm}$, suggesting existence of one equilibrium process. In addition, the peak position of OXP did not change indicating the preference of the added ligand for binding to $\mathrm{ZnP}$ and not OXP. The data was analyzed using the Benesi-Hildebrand method ${ }^{90}$ (Figure 5.3.2a inset) and a binding constant of $3.25 \times 10^{4}$ $\mathrm{M}^{-1}$ was obtained for the 1:1 complex. Interestingly, addition of $\mathrm{F}^{-}$(in the form of tetra- $n$ butylammonium fluoride) to a solution of ZnP-OXP lead to a red shift of the $515 \mathrm{~nm}$ band of OXP with an isosbestic point at $531 \mathrm{~nm}$ indicating $\mathrm{F}^{-}$binding to OXP. BenesiHildebrand analysis (Figure 5.3.2b inset) gave a binding constant of $2.36 \times 10^{5} \mathrm{M}^{-1}$ for the $1: 1$ complex, close to that reported for $\mathrm{F}^{-}$binding to the control compound, $\mathrm{N}, \mathrm{N}-$ bisalkyl substituted OXP $\left(1.2 \times 10^{5} \mathrm{M}^{-1}\right)$ but significantly larger than the value reported for $\mathrm{I}^{-}$binding to the control compound, being $3.5 \times 10^{3} \mathrm{M}^{-1} .{ }^{188}$ In another control 
experiment, ZnTPP was titrated with $\mathrm{F}^{-}$and a binding constant of $3.6 \times 10^{3} \mathrm{M}^{-1}$ was obtained (Figure 5.3.2c), which was nearly two orders of magnitude smaller than the value obtained for $\mathrm{F}^{-}$binding to OXP. These results collectively suggest preferential binding of nitrogenous ligand to the $\mathrm{ZnP}$ entity and $\mathrm{F}^{-}$to the OXP entity of the dyad; and binding preference of $\mathrm{F}^{-}$over $\mathrm{I}^{-}$to OXP.
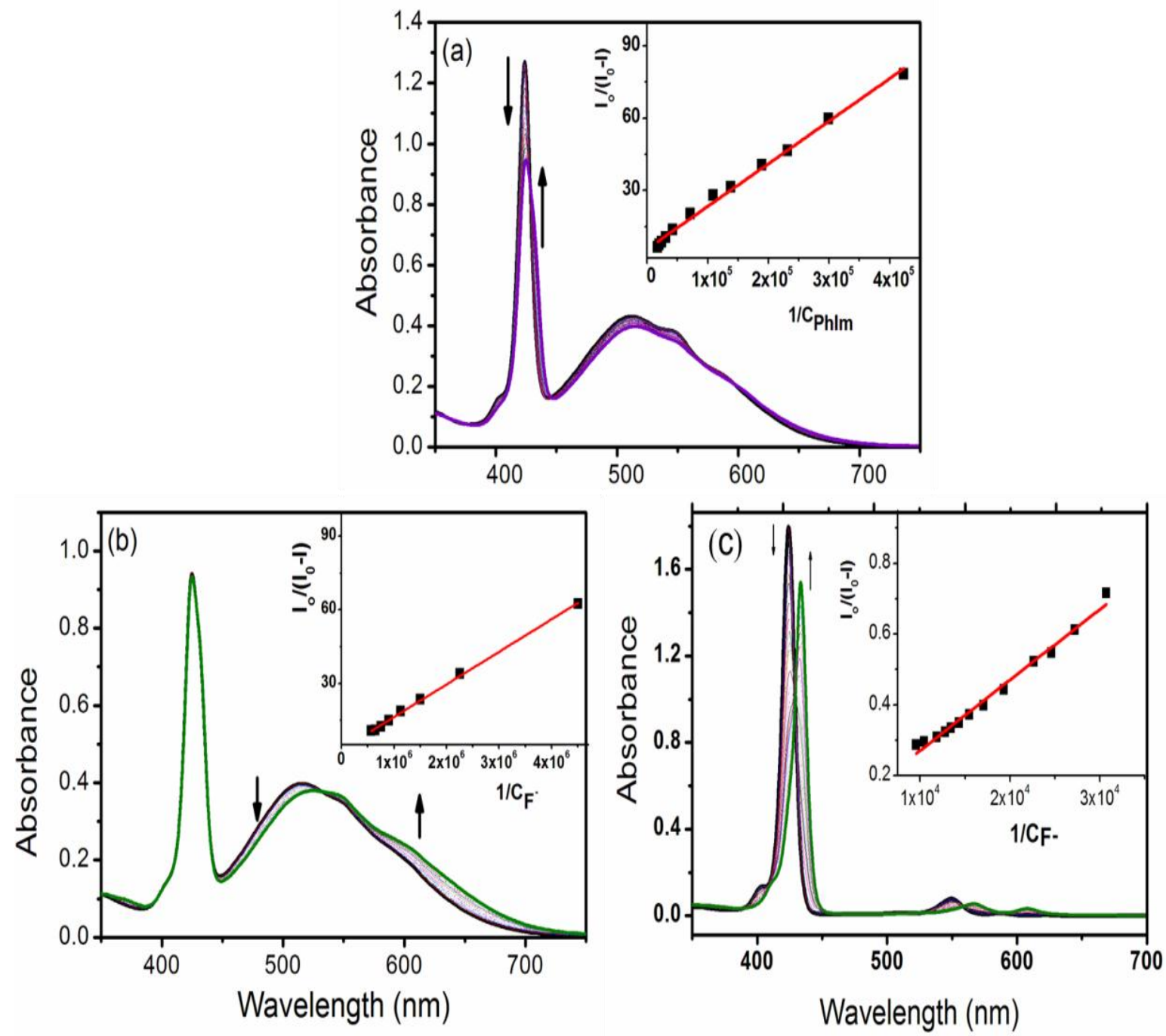

Figure 5.3.2: Absorption spectra of the ZnP-OXP dyad $(1.92 \mu \mathrm{M})$ upon increasing addition of (a) phenylimidazole axial coordinating ligand: (b) $\mathrm{F}^{-}(0.1-0.5$ eq.) in DCB: (c) Spectral changes observed during titration of $\mathrm{F}^{-}$into a solution of zinc tetraphenylporphyrin (ZnTPP) in DCB. The Benesi-Hildebrand plots constructed to obtain the binding constants for each equilibrium process are shown in the inset of the respective figures. 


\subsubsection{Electrochemical Studies}

Figure 5.3.3 shows differential pulse voltammograms (DPV) of ZnP-OXP dyad in DCB containing $0.10 \mathrm{M}(\mathrm{TBA}) \mathrm{ClO}_{4}$ in the presence and absence of added $\mathrm{F}^{-}$. Redox processes corresponding to both $\mathrm{ZnP}$ and $\mathrm{OXP}$ were observed. The site of electron transfer was determined by control experiments involving pristine ZnTPP and OXP-R $\left(\mathrm{R}_{1}=\mathrm{N} \text {-pyren-1-ylmethyl }\right)^{258}$ controls.

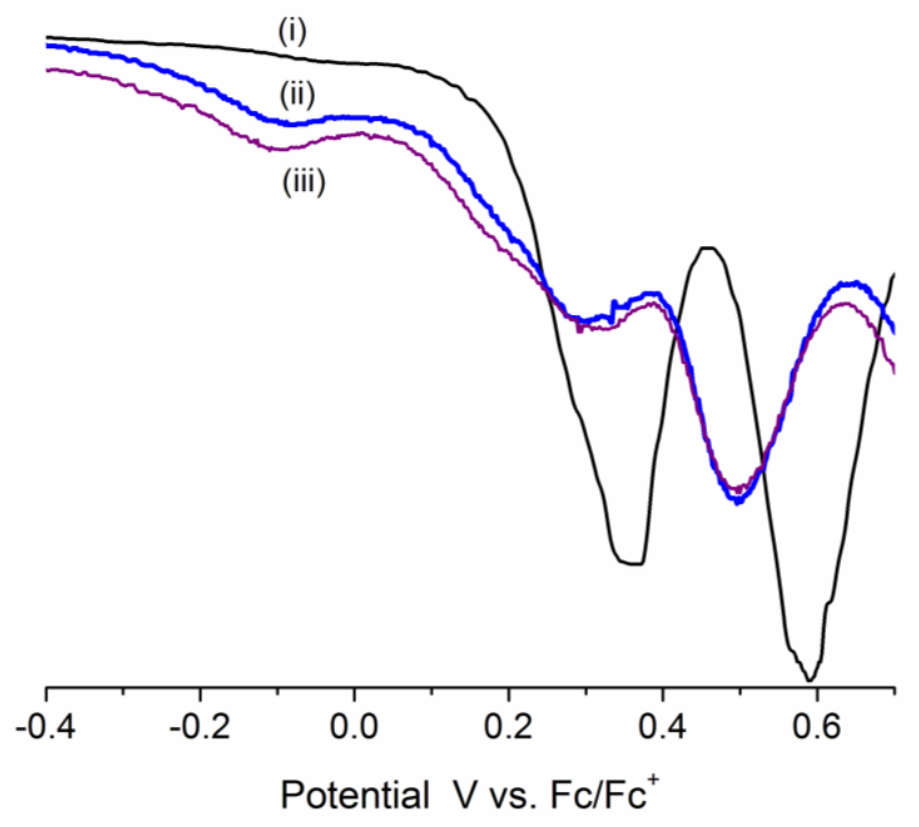

Figure 5.3.3: Differential pulse voltammograms of ZnP-OXP $(0.13 \mathrm{mM})$ (i) in the absence and (ii) and (iii) in the presence of $\mathrm{F}^{-}(1.3$ and $1.5 \mathrm{mM}$.) in deaerated DCB containing $0.10 \mathrm{M}(\mathrm{TBA}) \mathrm{ClO}_{4}$. Scan rate $=5 \mathrm{mV} / \mathrm{s}$, pulse width $=0.25 \mathrm{~s}$, pulse height $=$ $0.025 \mathrm{~V}$.

For OXP- $\mathrm{R}_{1}$, the first oxidation was located at $0.36 \mathrm{~V}$ vs. Fc/Fc ${ }^{+}$which shifted to $-0.20 \mathrm{~V}$ vs. $\mathrm{Fc} / \mathrm{Fc}^{+}$upon binding to $\mathrm{F}^{-}$. That is, a $560 \mathrm{mV}$ shift as a result of $\mathrm{F}^{-}$binding to OXP was observed. For the dyad, redox processes corresponding to first oxidation of ZnP was located at 0.28 (as a shoulder peak) while that corresponding to the mono $\mathrm{N}$ substituted OXP it was located at $0.35 \mathrm{~V}$ vs. $\mathrm{Fc} / \mathrm{Fc}^{+}$, and were not significantly different from pristine ZnTPP and OXP-R $\mathrm{R}_{1}$ control compounds. 
Notably, the first oxidation of $\mathrm{ZnP}$ was easier by $70 \mathrm{mV}$ than that of OXP. Addition of $\mathrm{F}^{-}$to the solution of the dyad gradually shifted the peak corresponding to OXP oxidation from $0.35 \mathrm{~V}$ to $E_{\mathrm{pa}}=-0.10 \mathrm{~V}$. That is, a nearly $450 \mathrm{mV}$ cathodic shift of OXP oxidation was observed upon $\mathrm{F}^{-}$addition. The second oxidation of the dyad located at $0.59 \mathrm{~V}$ (likely overlap of both $\mathrm{OXP}$ and $\mathrm{ZnP}$ oxidations) also revealed a cathodic shift with broadening and was shifted to $0.49 \mathrm{~V}$ vs. $\mathrm{Fc} / \mathrm{Fc}^{+}$. It may be noted here that no significant perturbation of the peak potential of the $\mathrm{ZnP}$ entity was observed upon $\mathrm{F}^{-}$addition to the dyad solution. The easier oxidation of $\mathrm{OXP}: \mathrm{F}^{-}$compared to $\mathrm{ZnP}$ by about $380 \mathrm{mV}$ makes it a hole shifting agent in the electron-transfer events as depicted in Scheme 5.3.1.

\subsubsection{Photoelectrochemical Studies}

Finally, photoelectrochemical cells were constructed to evaluate the effect of the bound $\mathrm{F}^{-}$on the current-voltage $(I-V)$ performance of $\mathrm{ZnP}-\mathrm{OXP}$ modified $\mathrm{TiO}_{2}$. For the cell construction, a thin non-crystalline $\mathrm{TiO}_{2}$ film coated fluorine doped indium tin oxide (FTO) electrode ( 10-12 microns, tec7 grade from Pilkington) was surface modified by placing the electrode in an ethanolic solution of 4-carboxyphenyl-imidazole $(0.20 \mathrm{M})$ overnight, according to our earlier published method. ${ }^{167}$ After removal of unbound material (2-3 ethanol washings), the $\mathrm{TiO}_{2}$ electrode was immersed in $\mathrm{DCB}$ solution containing ZnP-OXP for about 30 min. After this, the electrode was rinsed with DCB to remove excess of uncoordinated dyad. Photoelectrochemical cell assembly was completed using platinized FTO as counter electrode in non-coordinating DCB containing $0.50 \mathrm{M}(n-\mathrm{Bu})_{4} \mathrm{NI}$ and $0.030 \mathrm{M} \mathrm{I}_{2}$ as redox mediator Figure 5.3.4a shows typical $I-V$ plots for $\mathrm{ZnP}-\mathrm{OXP}$ modified $\mathrm{TiO}_{2}$ in the presence and absence of $\mathrm{F}^{-}$. In the 
case of $\mathrm{ZnP}-\mathrm{OXP}: \mathrm{F}^{-}$modified electrode, the photocurrent $I_{\mathrm{SC}}$ generated was nearly three times greater than that observed for ZnP-OXP. Although the reason is not very clear at present, the photovoltage $V_{\mathrm{OC}}$ was found to be slightly larger for the $\mathrm{ZnP}$ OXP: $\mathrm{F}^{-}$electrode by about $100 \mathrm{mV}$. The percent incident photon-to-current efficiency, ${ }^{4}$ IPCE(\%) plots for the electrodes are shown in Figure 5.3.4b. The shapes were found to be different from the absorbance spectra of the dyad in which only peaks corresponding to $\mathrm{ZnP}$ entity are observed. This indicates that the $\mathrm{ZnP}$ entity of the dyad is solely responsible for photocurrent generation and that the OXP: $\mathrm{F}^{-}$entity acts only as a hole transferring agent to slow down the charge recombination process, as discussed in the preceding section. The IPCE(\%) at the Soret band position was found to be $2 \%$ and $4 \%$ for $\mathrm{ZnP}-\mathrm{OXP}$ and $\mathrm{ZnP}-\mathrm{OXP}: \mathrm{F}^{-}$electrodes. Additionally, photo-switching on-off experiments revealed reproducible photocurrent generation (Figure 5.3.4b inset) for the electrode both in the absence and presence of added $\mathrm{F}^{-}$. These results indicate the robustness of the photocells constructed based on this supramolecular approach involving more than one equilibrium process (axial coordination and anion binding). As a control, a photocell was constructed using ZnTPP instead of ZnP-OXP, and photoelectrochemical behavior was recorded in the absence and presence of $\mathrm{F}^{-}$and no improvements in the photocurrents were observed indicating that the photocurrent enhancement is indeed due to redox modulating as a result of $\mathrm{F}^{-}$binding to OXP. 

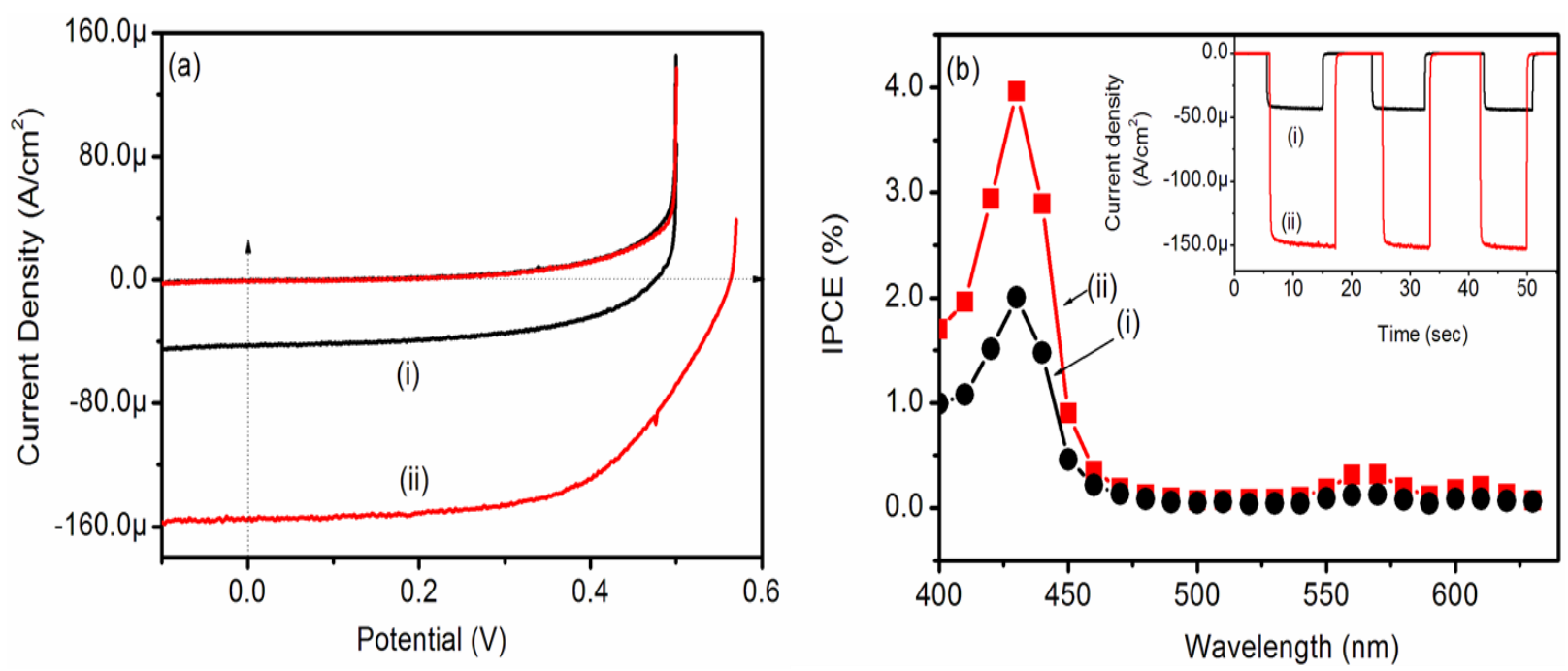

Figure 5.3.4: (a) $I-V$ and (b) IPCE(\%) characteristics revealing the effect of $\mathrm{F}^{-}$binding to $\mathrm{ZnP}-\mathrm{OXP}$ dyad (curves (i) in the absence and (ii) in the presence of $0.01 \mathrm{M} \mathrm{F}$ ). I-V curves were generated in DCB containing $\mathrm{I}_{3}^{-} / \mathrm{I}^{-}(0.50 \mathrm{M} / 0.050 \mathrm{M})$ redox mediator using an AM 1.5 simulated light source with a $340 \mathrm{~nm}$ UV-cut off filter. Figure b inset shows on-off light switching to demonstrate robustness of the studied electrode.

\subsubsection{Summary}

We have designed novel supramolecular solar cells to demonstrate anion binding induced enhancement of photocurrent generation. For this, thin-film nanocrystalline $\mathrm{TiO}_{2}$ was surface modified through metal-ligand axial coordination of a ZnP-OXP dyad. The photocells exhibited improved photocurrent conversion efficiency upon $\mathrm{F}^{-}$binding to the OXP entity. The easier oxidation of $\mathrm{OXP}: \mathrm{F}^{-}$compared to $\mathrm{ZnP}$ facilitated the electron transfer and hole migration to improve the phoelectrochemical performance. 


\section{CHAPTER 6}

\section{ION-PAIRED DYE-METAL OXIDE SUPRAMOLECULAR SYSTEM ${ }^{\dagger \dagger}$}

\subsection{Introduction}

Dye sensitized solar cells (DSSCs) $)^{259,260}$ have long been regarded as a promising alternative to conventional solid-state semiconductor solar cells, on the basis of their relatively high efficiency at a competitively low cost. ${ }^{46,154,156,261-270}$ A considerable number of inorganic/organic structures have been designed, synthesized and studied as molecular sensitizers ${ }^{74,176,265,271-276}$ with a combination of various band gap semiconductor nanoporous materials.$^{75,277-281}$ DSSCs based on Ru-complex dyes chemisorbed onto mesoporous titania with the help of carboxylate functionalities are shown to produce a photoelectric conversion yield of $12 \%$ under standard AM 1.5 light irradiation conditions. ${ }^{259,260}$ Lately, organic DSSCs have received more attention because of their ease of synthesis, high molar extinction coefficient and low cost in comparison to Ru-complexes. ${ }^{74,176,272-274,282-286}$ Additionally, by modifying the macrocycle periphery of the dye molecules it is possible to derive mechanisms for easy immobilization of the sensitizer molecules on nanocrystalline surface.

In recent years, researchers are also looking into replacing the commonly employed mesoporous $\mathrm{TiO}_{2}$ with other semiconducting materials. ${ }^{75,277-281}$ Among the possibilities, nanocrystalline $\mathrm{SnO}_{2}$ semiconductor is an attractive candidate as it possesses the conduction band lower by $\sim 0.4 \mathrm{~V}$ than $\mathrm{TiO}_{2}$, facilitating relatively efficient charge injection from the photoexcited dye molecule to semiconductor; ${ }^{88,287-293}$ although

\footnotetext{
${ }^{\dagger \dagger}$ Section 6 in its entirety is reproduced from the ACS Applied Materials and Interfaces, 2011, 3(7), pp
} 2368- 2376 with permission from the publisher. 
lower open-circuit voltage $\left(V_{O C}\right)$ could be expected due to the low lying conduction band. ${ }^{286,294}$ In this regard, some efforts to improve the $V_{\mathrm{OC}}$ of $\mathrm{SnO}_{2}$ based photoelectrochemical cells by surface treatment of $\mathrm{MgO}, \mathrm{ZnO}, \mathrm{TiCl}_{4}$ and several insulator oxides have been explored. ${ }^{295-297}$

Exploitation of the surface properties of nanocrystalline materials to immobilize appropriately functionalized photosensitizers in a self-assembled supramolecular approach is a relatively easy yet an elegant and versatile approach. ${ }^{167}$ Here, we report self-assembling cationic water soluble porphyrins onto thin-film nanocrystalline $\mathrm{SnO}_{2}$ surface via electrostatic interactions, and photoelectrochemical studies to evaluate their photon-to-current conversion efficiencies. The nanocrystalline $\mathrm{SnO}_{2}$ colloidal particles with negatively charged surface used in the present study have a particle diameter in the range of $30-50 \AA .^{88,287-293}$ Free-base and zinc (II) derivatives of three types of water soluble cationic porphyrins in which the positive charges progressively move far from the porphyrin $\pi$-ring (Scheme 1) have been used to decorate the $\mathrm{SnO}_{2}$ surface. The binding of cationic porphyrins onto the $\mathrm{SnO}_{2}$ surface were investigated by optical absorption studies while the excited state events from the singlet excited porphyrin was monitored using both steady-state and time-resolved emission techniques. The reversibility of electrostatic binding of $(\mathrm{P}) \mathrm{M}$ to $\mathrm{SnO}_{2}$ was established by varying ionic strength of the solution. Finally, photoelectrochemical studies were performed to evaluate $I-V$ characteristics and incident photon-to-current conversion (IPCE) efficiencies. As demonstrated here, the DSSC built using $\mathrm{FTO} / \mathrm{SnO}_{2} /$ cation porphyrin electrodes with $1^{-} / l_{3}^{-}$redox mediator in acetonitrile showed very high IPCE values, in some cases, as high as $91 \%$. Electrochemical impedance studies revealed decrease in 
recombination resistance under illumination due to increased local concentration of $\mathrm{I}_{3}{ }^{-}$ ions near to the dye electrode-electrolyte interface as a result of regeneration of dye molecule.

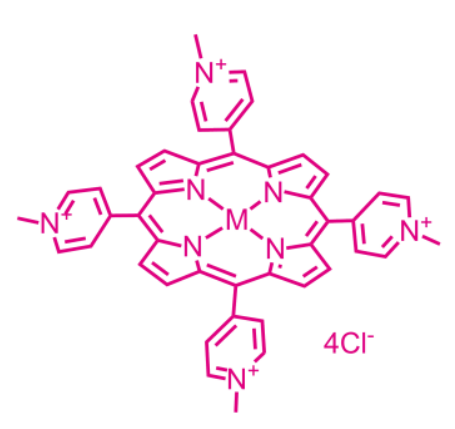

(TMPyP)M; M = 2H, Zn

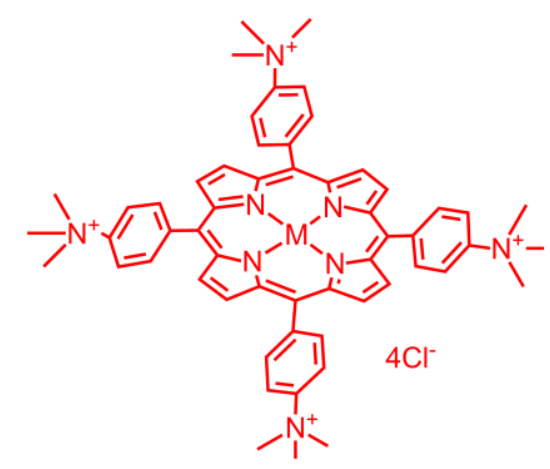

(TAP)M; $\mathrm{M}=2 \mathrm{H}, \mathrm{Zn}$

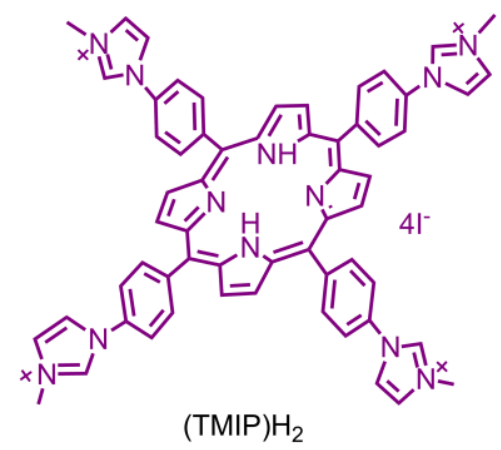

Scheme 6.1: Structures of the cationic water soluble porphyrins employed to surface modify nanocrystalline $\mathrm{SnO}_{2}$ for photoelectrochemical studies in the present study.

\subsection{Experimental Methods}

\subsubsection{Chemicals}

The free-base and zinc(II) derivatives of tetrakis( $\mathrm{N}$-methyl pyridyl)porphyrin chloride, (TMPyP)M, and tetrakis(trimethylanilinium)porphyrin chloride, (TAP)M were procured from Frontier Scientific, Inc. (Logan UT) and used as received. The synthesis of tetrakis(4'-N-methylimidazolylphenyl)porphyrin iodide, (TMIP) $\mathrm{H}_{2}$ is given below and was prepared by Dr. Eranda Maligaspe. Millipore water was used in all of the experiments. 
5,10,15,20-tetrakisphenylimidazolium porphyrin (1). To a $200 \mathrm{~mL}$ of propionic acid, $5.8 \mathrm{mmol}(1.0 \mathrm{~g})$ of $4-(1 \mathrm{H}$-imidazol-1-yl)benzaldehyde and $5.8 \mathrm{mmol}$ of pyrrole (452 $\mathrm{mL}$ ) were added. The solution was refluxed for $6 \mathrm{hrs}$ and the solvent was removed under reduced pressure. The crude was purified on a basic alumina column chromatography with $\mathrm{CHCl}_{3} / \mathrm{MeOH}(92: 8 \mathrm{v} / \mathrm{v})$ as eluent. ${ }^{1} \mathrm{H} \mathrm{NMR}\left(400 \mathrm{MHz}, \mathrm{CDCl}_{3}\right)$ (in ppm): $\delta 8.82$ (br s, $8 \mathrm{H}, \beta$ pyrrole), $8.28(\mathrm{~d}, 8 \mathrm{H}$, phenyl $H$ ) $8.16(\mathrm{~s}, 4 \mathrm{H}$, imidazole $H$ ), 7.78 $(\mathrm{d}, 8 \mathrm{H}$, phenyl $H$ ), $7.58(\mathrm{~s}, 4 \mathrm{H}$, imidazole $H), 7.25(\mathrm{~s}, 4 \mathrm{H}$, imidazole $H$ ). Mass (APCl mode in $\mathrm{CH}_{2} \mathrm{Cl}_{2}$ ): calcd, 879.5; found, 880.4.

5,10,15,20-tetrakis $\left(N\right.$-methylimidazolium-3-yl)porphyrin, (TMIP) $\mathrm{H}_{2}$. Compound 1 (0.025 mmol) was treated with $\mathrm{CH}_{3} \mathrm{l}(2.50 \mathrm{mmol})$ in $\mathrm{THF}(4 \mathrm{~mL})$, and reaction mixture was refluxed for 3 days. The solvent was removed, and the residue was recrystallized $\left(\mathrm{CH}_{3} \mathrm{OH} /\right.$ acetone). The obtained purple solid was gel chromatographed on a Sephadex $\mathrm{LH}-60$ using $\mathrm{CH}_{3} \mathrm{OH}$ as the eluent to give a purple product. ${ }^{1} \mathrm{H} \mathrm{NMR}(400 \mathrm{MHz}, \mathrm{MeOD})$ (in ppm): $\delta 8.95$ (br s, $8 \mathrm{H}, \beta$ pyrrole), 8.28 (d, $8 \mathrm{H}$, phenyl $H) 8.18(\mathrm{~s}, 4 \mathrm{H}$, imidazole $H$ ), $7.82(\mathrm{~d}, 8 \mathrm{H}$, phenyl $H$ ), $7.75(\mathrm{~s}, 4 \mathrm{H}$, imidazole $H),-7.21(\mathrm{~s}, 4 \mathrm{H}$, imidazole $H), 3.45(\mathrm{~s}$, $\left.12 \mathrm{H},-\mathrm{CH}_{3}\right)$.

\subsection{Results and Discussion}

\subsubsection{Optical Studies}

Binding of cationic water soluble porphyrins to nanocrystalline $\mathrm{SnO}_{2}$. Porphyrins, (TMPyP)M and (TAP)M ( $\mathrm{M}=2 \mathrm{H}$ or $\mathrm{Zn}(\mathrm{II}))$, used in the present study were procured from commercial sources while $(\mathrm{TMIP}) \mathrm{H}_{2}$ was newly synthesized. The optical absorption spectra of the investigated porphyrins in water are shown in Figure 6.1. The free-base porphyrin derivatives revealed the anticipated intense Soret and four visible 
(Q) bands while for the corresponding zinc derivatives, only two visible bands in addition to the intense Soret was observed due to the increased symmetry upon metal ion insertion into the ring cavity (Table 6.1).

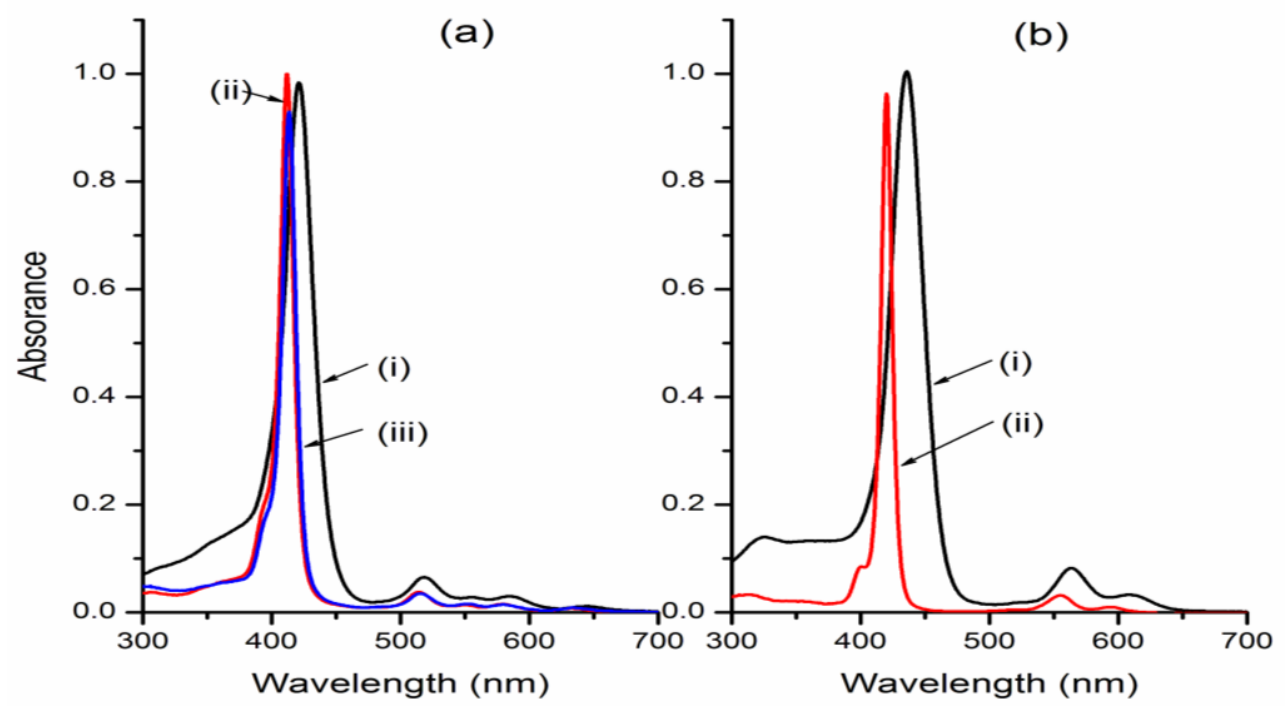

Figure 6.1: Absorption spectra of the (a) free-base and (b) zinc derivatives of (i) TMPyP, (ii) TAP and (iii) TMIP porphyrin derivatives normalized to the Soret band intensity

The peak maxima of (TMPyP)M derivatives revealed a red shift of up to $6 \mathrm{~nm}$ compared to the peak maxima of (TAP)M and (TMIP)M derivative. Additionally, the full width at half maxima (FWHM) values for the former derivatives was found to be over 3 times as much as that of the latter derivatives, that is, substantial broadening of the absorption peaks were observed for the (TMPyP)M derivatives. The red shift accompanied by broadening of absorption bands for (TMPyP)M derivatives has earlier been ascribed to aggregate formation with substantial interaction between the porphyrin ring $\pi$-system with peripheral positive charges. ${ }^{298,299}$ Based on the spectral features of (TAP)M and (TMIP)M derivatives one could conclude presence of little or absence of aggregation and interactions with the peripheral positive charges. This may primarily due to unfavorable geometry and distant location of the positive charges from the 
macrocycle $\pi$-system. As mentioned earlier, the diameter of the $\mathrm{SnO}_{2}$ nanocrystalline colloidal particles used here are in the range of $30-50 \AA$ suggesting their ability to accommodate one or more relatively large photosensitizer porphyrin molecules on the surface. Figure 6.6a shows spectral changes observed during increased addition of $\mathrm{SnO}_{2}$ colloidal to a solution of $(\mathrm{TMPyP}) \mathrm{H}_{2}$ in water. Similar spectral changes were observed for the investigated free-base and zinc porphyrin derivatives (see Figures 6.2 to 6.5). The absorbance of $\mathrm{SnO}_{2}$ was seen at wavelength less than $300 \mathrm{~nm}$. Addition of $\mathrm{SnO}_{2}$ caused diminished intensity of both the Soret and the visible bands. As shown in Figure $1 \mathrm{~b}$ formation of interaction between porphyrin and $\mathrm{SnO}_{2}$ was accompanied by a red shift of 22-26 $\mathrm{nm}$ for the TMPyP derivatives, and 12-17 $\mathrm{nm}$ for the TAP and TMIP derivatives, respectively. Earlier, the large red-shift and broadening of TMPyP upon adsorption on laponite surface (negatively charged polyionic platelets) was attributed to the flattening of the porphyrin macrocycle due to pyridyl torsion. ${ }^{300}$ Such a structural change is also expected for (TMPyP)M binding to $\mathrm{SnO}_{2}$ particles. Additionally, 2-4 isosbestic points were observed indicating the existence of one equilibrium process in solution. 

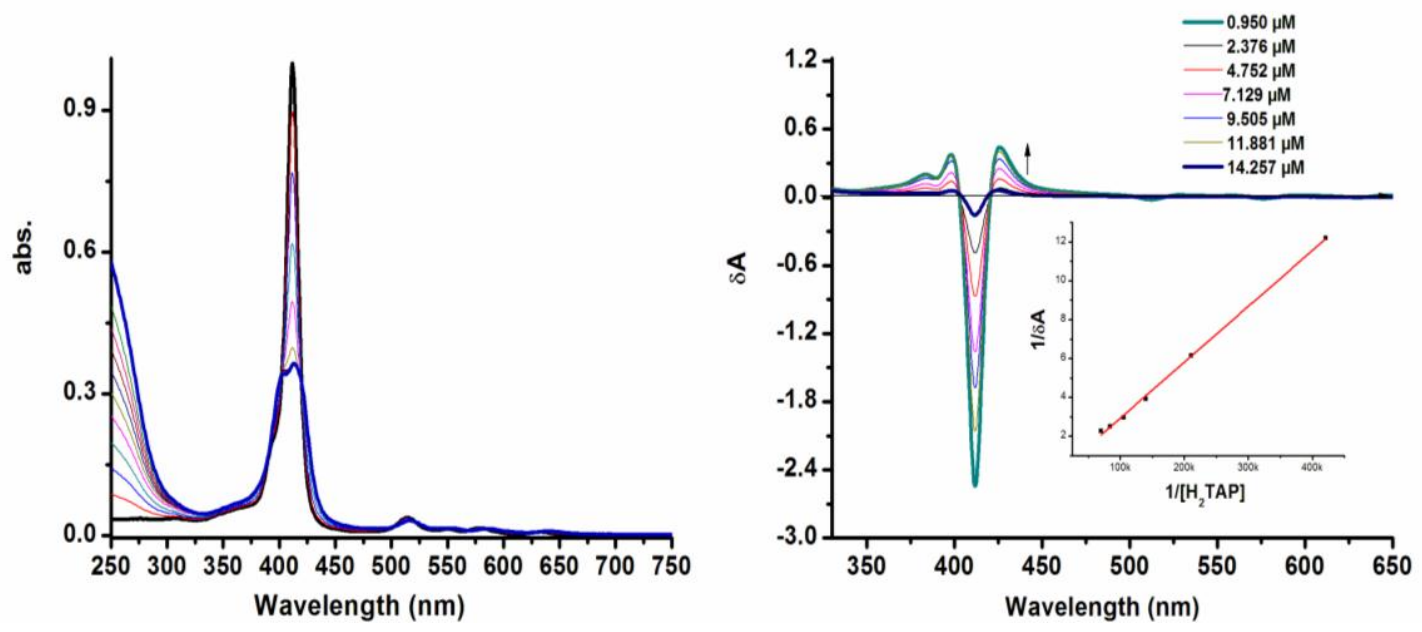

Figure 6.2: Absorption spectral changes observed for (TPA) $\mathrm{H}_{2}$ on increasing addition of colloidal $\mathrm{SnO}_{2}$ (5-10 $\mu \mathrm{L}$ of $1.5 \mathrm{~g} / \mathrm{l}$ each addition) in water (left panel). Absorption spectra recorded with two-beam spectrometer during addition of aqueous solution of (TPA) $\mathrm{H}_{2}$ (5 $\mu \mathrm{L}$ of $1.43 \mathrm{mM}$ each addition) to a $\mathrm{SnO}_{2}(3 \mathrm{ml}$ of $0.408 \mathrm{~g} / \mathrm{l})$ solution in water (right panel). $\delta A=A-A o$, where $A$ and $A o$ corresponds to the absorbance of (TPA) $\mathrm{H}_{2}$ in the presence and absence of $\mathrm{SnO}_{2}$. Figure inset in the right panel shows BenesiHildebrand plot showing linear dependence of the inverse $\delta A$ at $427 \mathrm{~nm}$ on the inverse of the (TPA) $\mathrm{H}_{2}$ concentration
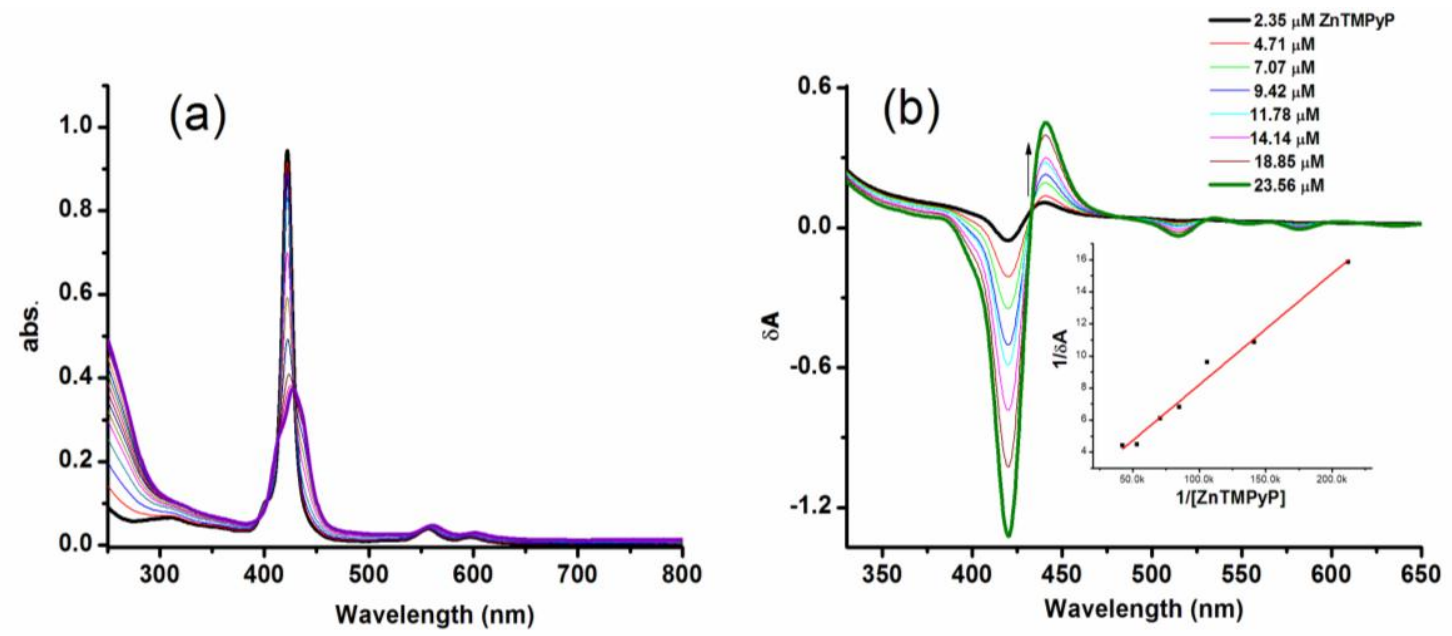

Figure 6.3: (a) Absorption spectral changes observed for (TMPyP)Zn on increasing addition of colloidal $\mathrm{SnO}_{2}(5-10 \mu \mathrm{L}$ of $1.2 \mathrm{~g} / \mathrm{l}$ each addition) in water. (b) Absorption spectra recorded with two-beam spectrometer during addition of aqueous solution of (TMPyP)Zn ( $5 \mu \mathrm{L}$ of $1.41 \mathrm{mM}$ each addition) to a $\mathrm{SnO}_{2}(3 \mathrm{ml}$ of $0.42 \mathrm{~g} / \mathrm{l})$ solution in water. $\delta \mathrm{A}=\mathrm{A}-\mathrm{Ao}$, where $\mathrm{A}$ and Ao corresponds to the absorbance of (TMPyP) $\mathrm{Zn}$ in the presence and absence of $\mathrm{SnO}_{2}$. Figure $2 \mathrm{~b}$ inset shows Benesi-Hildebrand plot showing linear dependence of the inverse $\delta A$ at $459 \mathrm{~nm}$ on the inverse of the (TMPyP)Zn concentration. 

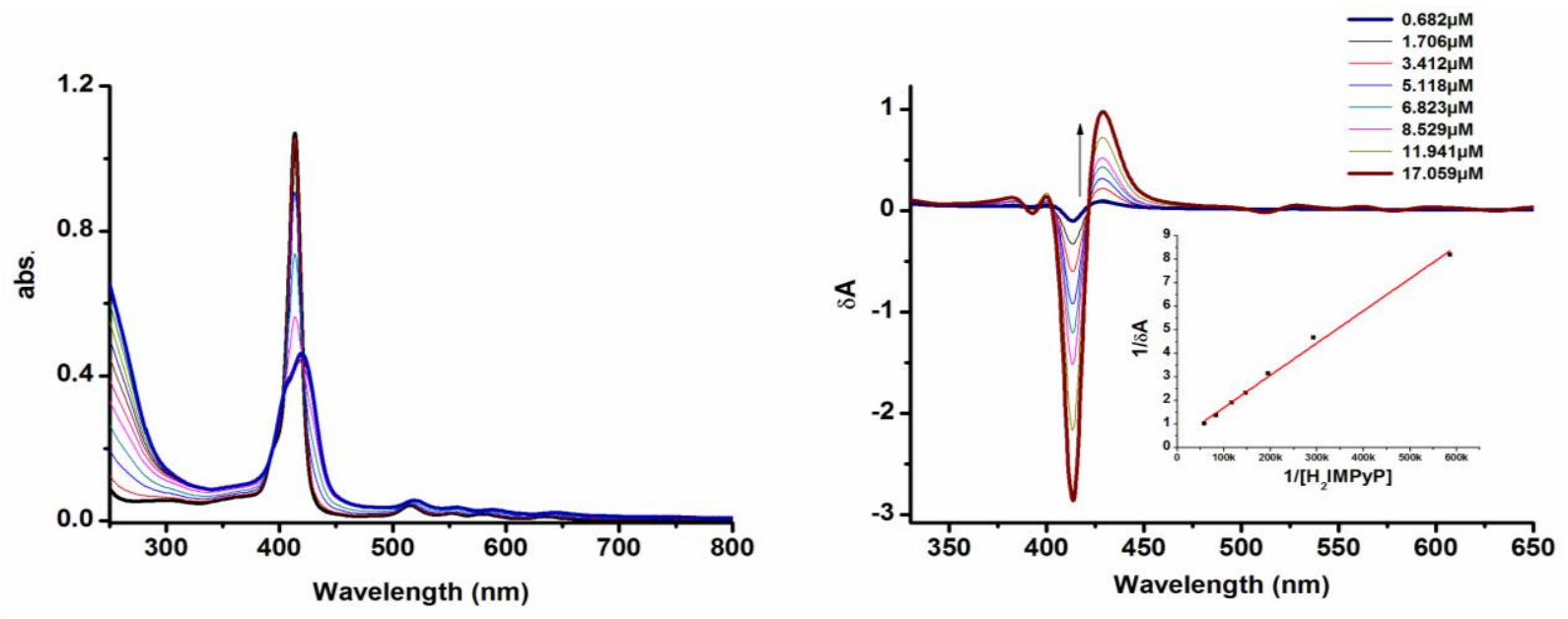

Figure 6.4: Absorption spectral changes observed for (TMIP) $\mathrm{H}_{2}$ on increasing addition of colloidal $\mathrm{SnO}_{2}(5-10 \mu \mathrm{L}$ of $1.5 \mathrm{~g} / \mathrm{l}$ each addition) in water (left panel). Absorption spectra recorded with two-beam spectrometer during addition of aqueous solution of (TMIP) $\mathrm{H}_{2}\left(5 \mu \mathrm{L}\right.$ of $1.02 \mathrm{mM}$ each addition) to a $\mathrm{SnO}_{2}(3 \mathrm{ml}$ of $0.412 \mathrm{~g} / \mathrm{l})$ solution in water (right panel). $\delta \mathrm{A}=\mathrm{A}-\mathrm{Ao}$, where $\mathrm{A}$ and Ao corresponds to the absorbance of $(\mathrm{TMIP}) \mathrm{H}_{2}$ in the presence and absence of $\mathrm{SnO}_{2}$. Figure right inset shows Benesi-Hildebrand plot showing linear dependence of the inverse $\delta A$ at $428 \mathrm{~nm}$ on the inverse of the (TMIP) $\mathrm{H}_{2}$ concentration
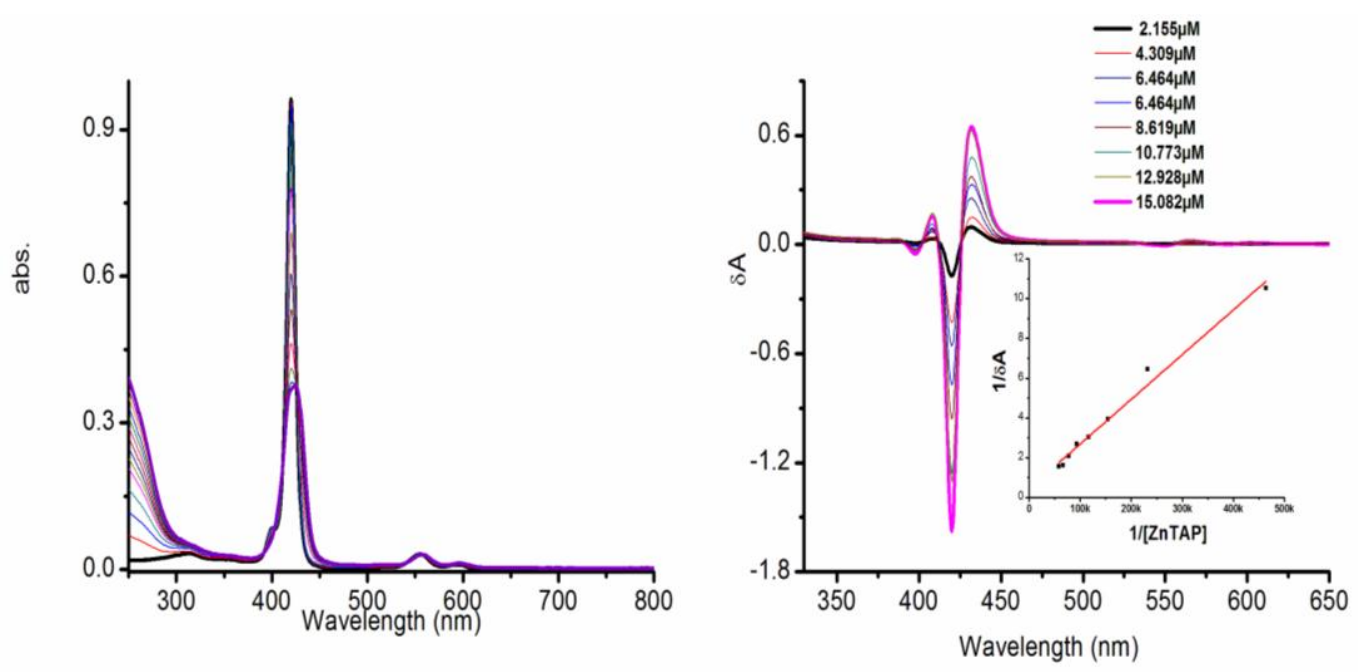

Figure 6.5: Absorption spectral changes observed for (TAP)Zn on increasing addition of colloidal $\mathrm{SnO}_{2}(5-10 \mu \mathrm{L}$ of $1.2 \mathrm{~g} / \mathrm{l}$ each addition) in water (left panel). Absorption spectra recorded with two-beam spectrometer during addition of aqueous solution of (TAP)Zn (5 $\mu \mathrm{L}$ of $1.3 \mathrm{mM}$ each addition) to a $\mathrm{SnO}_{2}(3 \mathrm{ml}$ of $0.40 \mathrm{~g} / \mathrm{l})$ solution in water (right panel). $\delta \mathrm{A}=\mathrm{A}-\mathrm{Ao}$, where $\mathrm{A}$ and Ao corresponds to the absorbance of (TAP)Zn in the presence and absence of $\mathrm{SnO}_{2}$. Figure right inset shows Benesi-Hildebrand plot showing linear dependence of the inverse $\delta A$ at $431 \mathrm{~nm}$ on the inverse of the (TAP)Zn concentration 

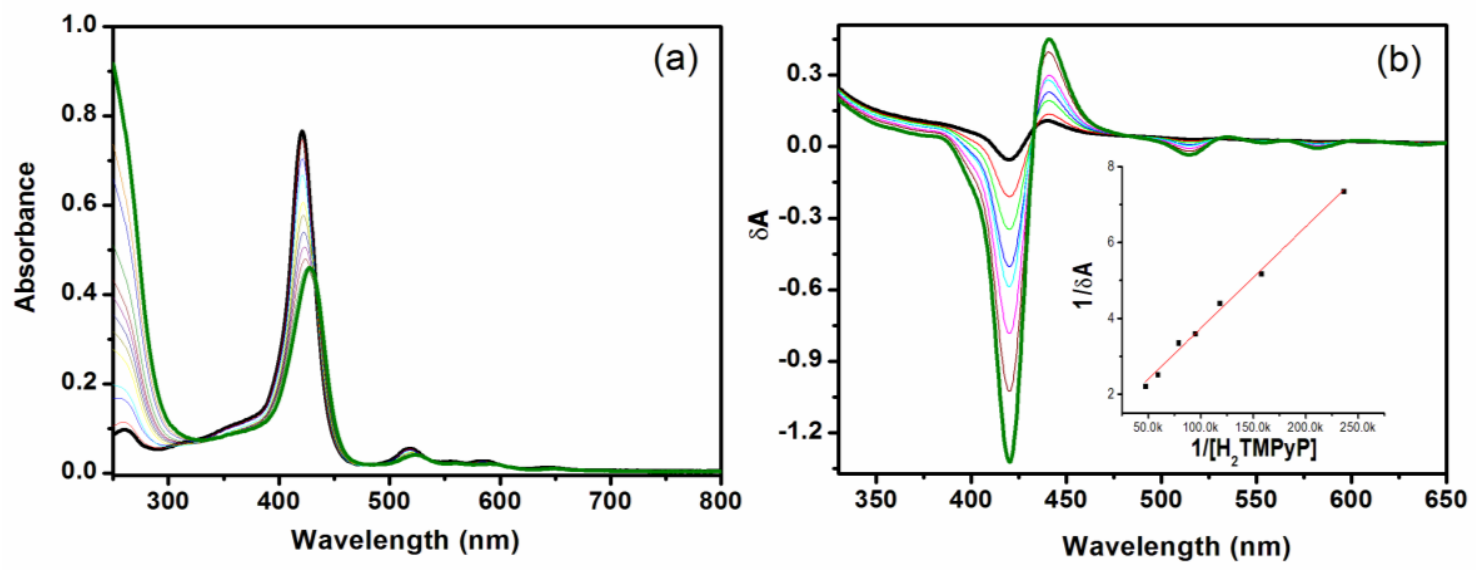

Figure 6.6: (a) Absorption spectral changes observed for (TMPyP) $\mathrm{H}_{2}(3 \mathrm{ml}$ of $2.17 \mathrm{mM})$ on increasing addition of colloidal SnO2 (5-10 $\mu \mathrm{L}$ of $11.2 \mathrm{mM}$ each addition) in water. (b) Absorption spectra recorded with two-beam spectrometer during addition of aqueous solution of (TMPyP) $\mathrm{H}_{2}\left(5 \mu \mathrm{L}\right.$ of $1.4 \mathrm{mM}$ each addition) to a $\mathrm{SnO}_{2}(3 \mathrm{ml}$ of $0.85 \mathrm{~g} / \mathrm{L})$ solution in water. $\delta \mathrm{A}=\mathrm{A}-\mathrm{Ao}$, where $\mathrm{A}$ and Ao corresponds to the absorbance of $(\mathrm{TMPyP}) \mathrm{H}_{2}$ in the presence and absence of $\mathrm{SnO}_{2}$. Figure $6.6 \mathrm{~b}$ inset shows BenesiHildebrand plot showing linear dependence of the inverse $\delta A$ at $449 \mathrm{~nm}$ on the inverse of the (TMPyP) $\mathrm{H}_{2}$ concentration.

Figure $6.6 \mathrm{~b}$ shows the difference in the absorption spectra of the $\mathrm{SnO}_{2}$ :(TMPyP) $\mathrm{H}_{2}$ complex. Here, a quartz cuvette with aqueous suspension of $\mathrm{SnO}_{2}$ in the sample beam and another cuvette with pure water in the reference beam were introduced in a dual beam spectrophotometer. Same amounts of $(\mathrm{TMPyP}) \mathrm{H}_{2}$ of known increments were added into both cuvettes and the spectrum was recorded. With increasing additions of (TMPyP) $\mathrm{H}_{2}$, increasing amounts of $\mathrm{SnO}_{2}$ :(TMPyP) $\mathrm{H}_{2}$ complex formation were observed as revealed by the absorption peak at $441 \mathrm{~nm}$ that was accompanied by depletion of the absorption of (TMPyP) $\mathrm{H}_{2}$ at $422 \mathrm{~nm}$ band. A plot of change in absorbance $\delta A$ at $441 \mathrm{~nm}$ vs. amount of (TMPyP) $\mathrm{H}_{2}$ added was linear (Figure $6.6 \mathrm{~b}$ inset) suggesting that porphyrin present in solution adsorbs on $\mathrm{SnO}_{2}$ as monomeric species. Such linear dependence was observed for all of the investigated porphyrin derivatives (Figures 6.2 to 6.5 ) suggesting monomeric porphyrin adsorbing 
onto $\mathrm{SnO}_{2}$ surface. Previously, a quadratic dependence of absorbance on concentration confirmed the a dimeric species adsorption onto the $\mathrm{SnO}_{2}$ surface as reported by Liu and Kamat using smaller cationic dyes, thionine, methylene blue or Ox170. ${ }^{288}$ The spectra in Figure $6.6 \mathrm{~b}$ was further analyzed by constructing BenesiHildebrand plot $^{90}$ as shown in Figure $6.6 \mathrm{~b}$ inset. Here, inverse of the difference in absorbance at $449 \mathrm{~nm}$ was plotted against inverse of the amount of added (TMPyP) $\mathrm{H}_{2}$. A linear plot was obtained; using the slope and intercept the apparent association constant, $K_{\mathrm{a}}$, was evaluated to be $1.5 \times 10^{4} \mathrm{M}^{-1}$. Similar values of $K_{\mathrm{a}}$ were obtained for rest of the water soluble, cation porphyrins binding to $\mathrm{SnO}_{2}$, as listed in Table 6.1. The $K_{\mathrm{a}}$ values found to follow the trend: (TMPyP)M $<$ (TAP)M $<$ (TMIP)M, suggesting stronger binding of (TMIP) $\mathrm{H}_{2}$, perhaps due to better geometry reasons. It may also be mentioned here that the $K_{\mathrm{a}}$ values reported here should be treated with some caution since varying size of $\mathrm{SnO}_{2}$ particles and aggregation of water soluble porphyrins especially (TMPyP)M derivatives, may restrict absolute determination of the binding constants.

Table 6.1: Spectral peak position and apparent association constant, $K a$ and freeenergy change for charge injection of water soluble, cation porphyrins binding to $\mathrm{SnO}_{2}$ nanocrystalline particles in water.

\begin{tabular}{cccc}
\hline $\mathrm{M}(\mathrm{P})$ & Peak position & $n K_{\mathrm{a}}, \mathrm{M}^{-1}$ & $\Delta G^{\mathrm{o}}{ }_{\mathrm{inj}}, \mathrm{eV}$ \\
\hline (TMPyP)Zn & $435,563,609$ & $1.8 \times 10^{4}$ & -0.77 \\
$(\mathrm{TAP}) \mathrm{Zn}$ & $419,554,594$ & $1.95 \times 10^{4}$ & -0.93 \\
$(\mathrm{TMPyP}) \mathrm{H}_{2}$ & $422,518,555,584,641$ & $1.5 \times 10^{4}$ & -0.44 \\
$(\mathrm{TAP}) \mathrm{H}_{2}$ & $411,513,549,579,633$ & $1.77 \times 10^{4}$ & -0.56 \\
$(\mathrm{TMIP}) \mathrm{H}_{2}$ & $414,514,551,579,634$ & $2.6 \times 10^{4}$ & -0.54 \\
\hline
\end{tabular}




\subsubsection{Fluorescence Quenching Studies}

The effect of semiconducting $\mathrm{SnO}_{2}$ particles on the fluorescence emission of the porphyrins was investigated using both steady-state and time-resolved studies. As shown in Figure 6.10a for the (TMPyP) $\mathrm{H}_{2}$ derivative, addition of $\mathrm{SnO}_{2}$ quenched the fluorescence emission over $70 \%$ of its original intensity accompanied by small red shifts. This was also the case for rest of the zinc and free-base porphyrin derivatives but the percentage of quenching was generally higher for the zinc derivatives (as much
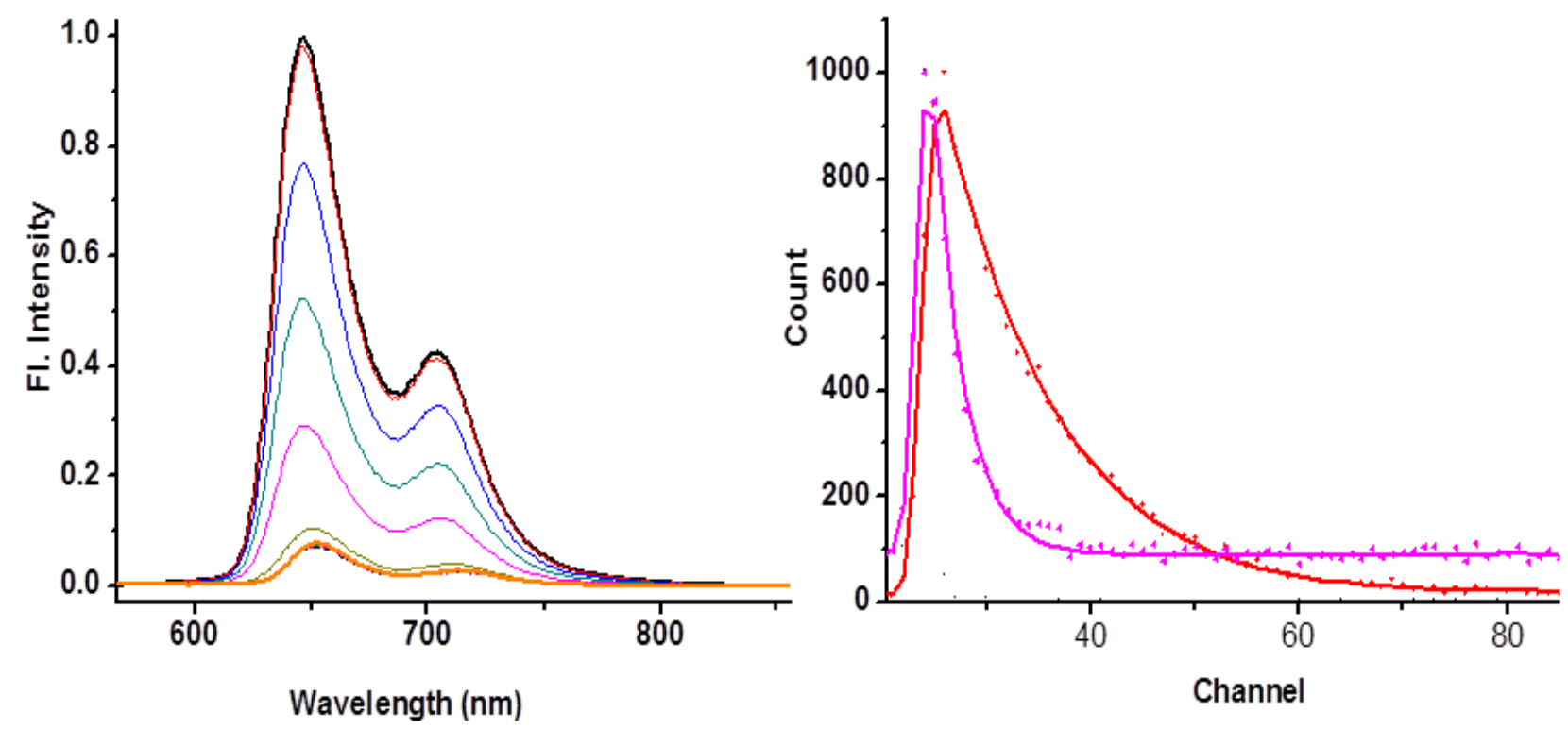

Figure 6.7: Fluorescence spectra of (TMIP) $\mathrm{H}_{2}$ on increasing addition nanocrystalline $\mathrm{SnO}_{2}\left(5-20 \mu \mathrm{L}\right.$ of $1.5 \mathrm{~g} / \mathrm{l}$ solution addition) (left panel). $\lambda_{\mathrm{ex}}=517 \mathrm{~nm}$. Fluorescence decay profiles of (TMIP) $\mathrm{H}_{2}$ in the absence (i) and presence (ii) of nanocrystalline $\mathrm{SnO}_{2}$, excited at $561 \mathrm{~nm}$ using a nano-LED source (right panel). The lamp profile is shown in dashed line. 

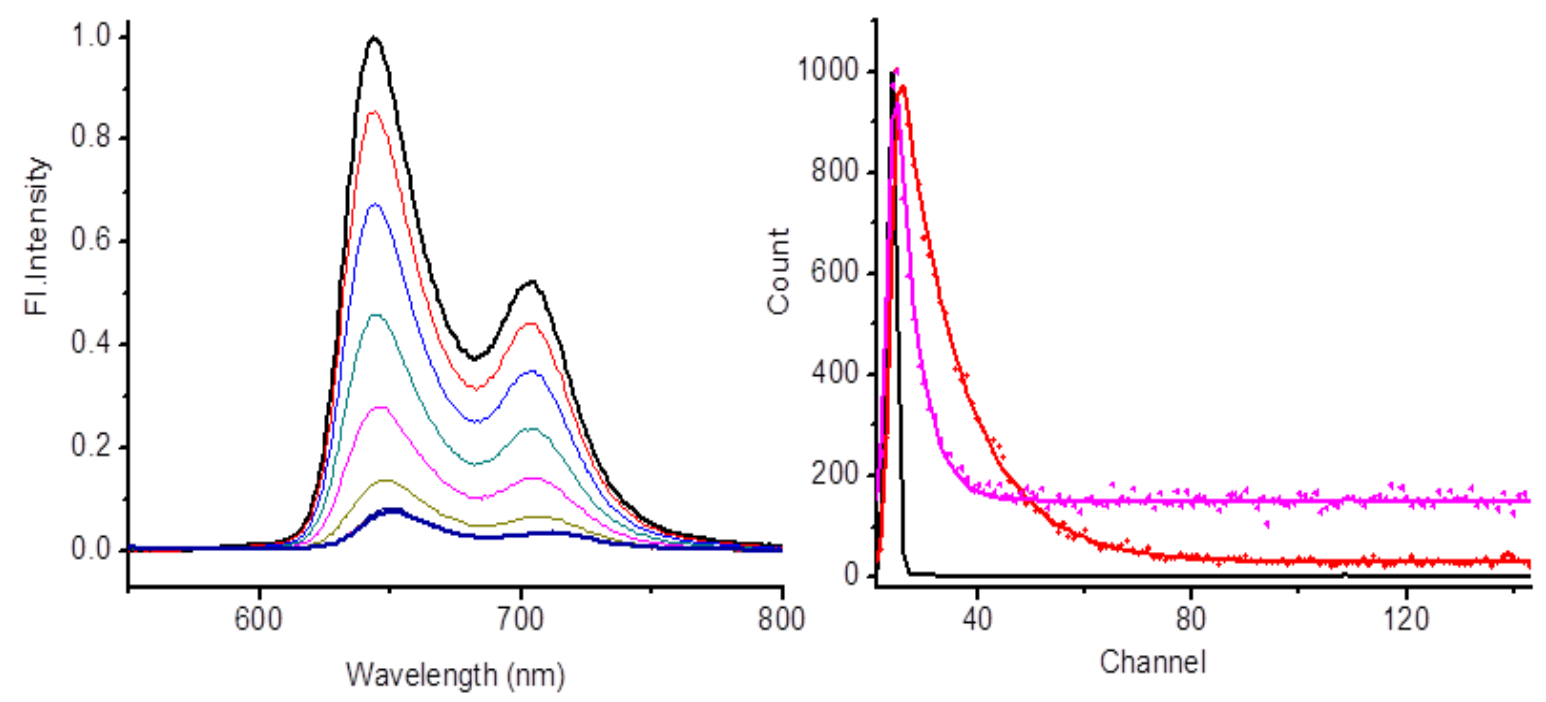

Figure 6.8: (a) Fluorescence spectra of (TAP) $\mathrm{H}_{2}$ on increasing addition nanocrystalline $\mathrm{SnO}_{2}\left(5-20 \mu \mathrm{L}\right.$ of $1.5 \mathrm{~g} / \mathrm{l}$ solution addition) (left panel). $\lambda_{\mathrm{ex}}=517 \mathrm{~nm}$. (b) Fluorescence decay profiles of (TAP) $\mathrm{H}_{2}$ in the absence (i) and presence (ii) of nanocrystalline $\mathrm{SnO}_{2}$, excited at $561 \mathrm{~nm}$ using a nano-LED source (right panel). The lamp profile is shown in dashed line.
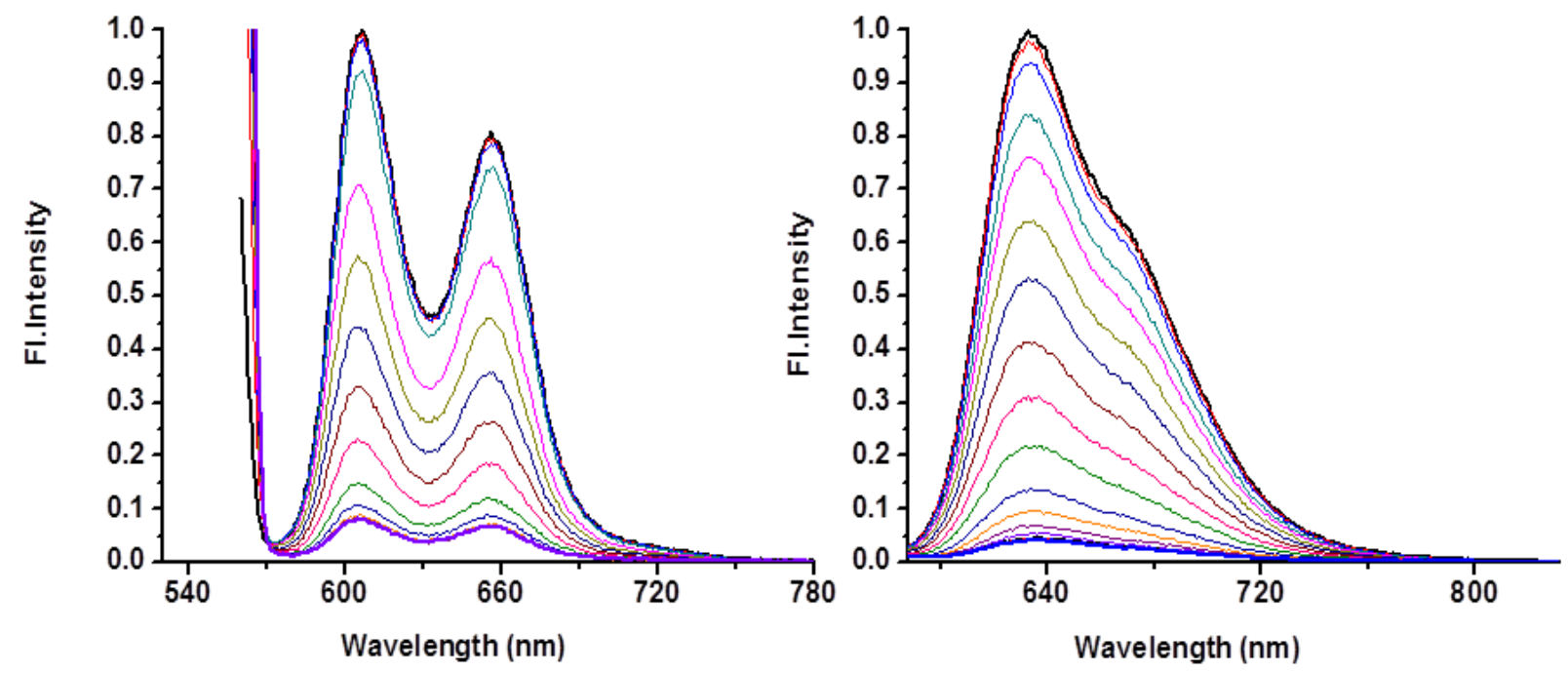

Figure 6.9: Fluorescence spectra of (TAP)Zn $(2.17 \mu \mathrm{M})$ on increasing addition nanocrystalline $\mathrm{SnO}_{2}\left(5-20 \mu \mathrm{L}\right.$ of $1.2 \mathrm{~g} / \mathrm{l}$ solution addition) (left panel). $\quad \lambda_{\mathrm{ex}}=563 \mathrm{~nm}$. Fluorescence spectra of (TMPyP)Zn $(2.17 \mu \mathrm{M})$ on increasing addition nanocrystalline $\mathrm{SnO}_{2}\left(5-20 \mu \mathrm{L}\right.$ of $1.2 \mathrm{~g} / \mathrm{l}$ solution addition) (left panel). $\lambda_{\mathrm{ex}}=563 \mathrm{~nm}$. (right panel). 
as $96 \%$ in the case of (TMPyP)Zn) compared to the free-base ones (see Figures 6.7 to 6.9). In order to clarify whether the quenching is due to heavy atom effect, control experiments involving fluorescence quenching of (TMPyP)Zn in the presence of $\mathrm{SnCl}_{2}$ were performed. Presence of $\mathrm{SnCl}_{2}$ quenched the fluorescence by less than $5 \%$ suggesting heavy atom quenching is not a likely quenching mechanism. Further, the lifetime of free-base porphyrins were measured with incremental addition of $\mathrm{SnO}_{2}$. The emission of both $(\mathrm{TAP}) \mathrm{H}_{2}$ and $(\mathrm{TMIP}) \mathrm{H}_{2}$ revealed monoexponential decays with lifetimes of 9.14 and $9.96 \mathrm{~ns}$, respectively, when excited using a $561 \mathrm{~nm}$ nano-LED source. However, (TMPyP) $\mathrm{H}_{2}$ revealed a biexponential decay with lifetimes of 5.2 (85\%) and $1.2(15 \%)$ ns yielding an average lifetime of 4.7 ns (Figure $6.10 \mathrm{~b}$ ), a result that agrees well with the literature value. ${ }^{301}$ The decreased life time for TMPyP may be attributed to their strong intermolecular aggregation in solution. Addition of $\mathrm{SnO}_{2}$ to the solution induced rapid decay and at the saturation point, the lifetimes of (TAP) $\mathrm{H}_{2}$ and (TMIP) $\mathrm{H}_{2}$ were found to be 2.47 and $3.51 \mathrm{~ns}$, respectively, while the average lifetime of $(\mathrm{TMPyP}) \mathrm{H}_{2}$ in the presence of $\mathrm{SnO}_{2}$ was $3.5 \mathrm{~ns}$. The percent quenching by timeresolved emission were generally smaller compared to steady-state measurements indicating the presence of both static and dynamic quenching. ${ }^{301}$ It may be mentioned here that we could not measure the lifetimes of the zinc porphyrins due to low time resolution of the instrument.

In order to verify that the excited state electron transfer is the quenching mechanism, the standard free energy change for electron injection, $\Delta G^{\circ}{ }_{\text {inj }}$ was estimated from the redox potentials of the donor porphyrins, ${ }^{302,303}$ acceptor $\mathrm{SnO}_{2}$ particles $^{294}$ and singlet state energies of the employed porphyrins ${ }^{294}$ according to 
literature methods. ${ }^{304}$ Such data are given in Table 6.1 and its dependence on metal ion in the porphyrin cavity clearly shows that charge injection from the singlet excited porphyrin to $\mathrm{SnO}_{2}$ is a likely the quenching mechanism for all of the employed porphyrins.

By assuming the quenching is due to charge injection from the singlet excited porphyrin to conduction band of $\mathrm{SnO}_{2}$, the rate constants were estimated according to equation $1 .{ }^{149}$

$$
k=1 / \tau_{\text {bound }}-1 / \tau_{\text {free }}
$$

where $\tau_{\text {free }}$ and $\tau_{\text {bound }}$ represent lifetime of porphyrin in the absence of presence of $\mathrm{SnO}_{2}$, respectively. The rates thus measured were found to be $2.95 \times 10^{8} \mathrm{~s}^{-1}, 1.84 \mathrm{x}$ $10^{8} \mathrm{~s}^{-1}$ and $0.73 \times 10^{8} \mathrm{~s}^{-1}$, respectively for fluorescence quenching of (TAP) $\mathrm{H}_{2},\left(\right.$ TMIP) $\mathrm{H}_{2}$ and $(\mathrm{TMPyP}) \mathrm{H}_{2}$ by $\mathrm{SnO}_{2}$. The magnitude of $k$ values suggest rapid charge injection and that the free-base porphyrin derivatives with peripheral positive charges away from the $\pi$-system are better candidates for charge injection, perhaps due to slightly easier oxidation of the latter porphyrin macrocyle. ${ }^{303}$ However, other factors may also play important role in governing the overall efficiency as discussed in the forthcoming section. 

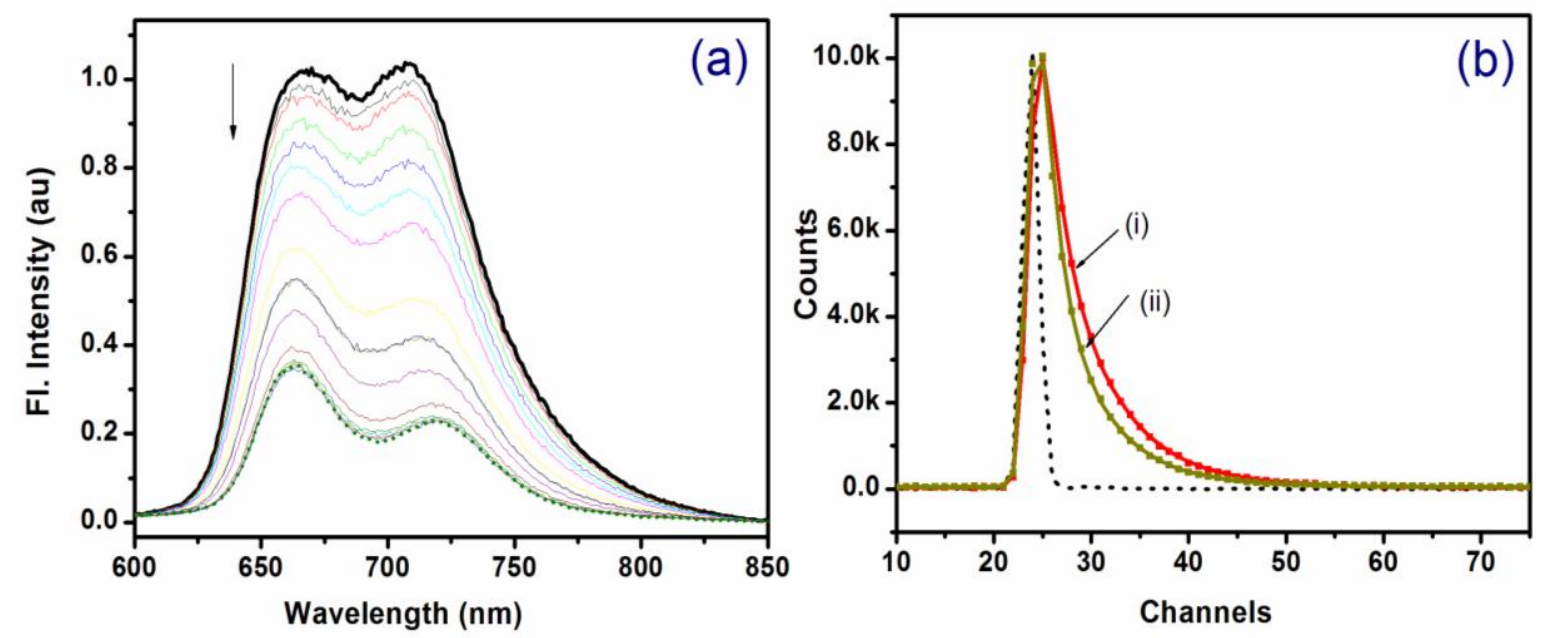

Figure 6.10: (a) Fluorescence spectra of $\left(\mathrm{TMPyP}^{\mathrm{H}} \mathrm{H}_{2}(3 \mathrm{ml}\right.$ of $2.17 \mu \mathrm{M})$ on increasing addition nanocrystalline $\mathrm{SnO}_{2}$ (addition of $5-20 \mu \mathrm{L}$ solution of $1.1 \mathrm{~g} / \mathrm{L}$ concentration). $\lambda_{\text {ex }}$ $=517 \mathrm{~nm}$. (b) Fluorescence decay profiles of (TMPyP) $\mathrm{H}_{2}$ in the absence (i) and presence (ii) of nanocrystalline $\mathrm{SnO}_{2}$, excited at $561 \mathrm{~nm}$ using a nano-LED source. The lamp profile is shown in dashed line. Time calibration factor for each channel $=8.77 \mathrm{x}$ $10^{-10} \mathrm{~s}$.

Additional experiments were performed to visualize the stability of electrostatically adsorbed porphyrin dyes onto the $\mathrm{SnO}_{2}$ surface by monitoring the fluorescence recovery by increasing addition of $\mathrm{LiClO}_{4}$ to the solution. ${ }^{305}$ In the case of (TAP)M, and (TMPyP)M up to $80 \%$ of the quenched porphyrin intensity could be recovered upon addition of $\mathrm{LiClO}_{4}$ (Figure 6.11) However, for (TMIP) $\mathrm{H}_{2}$ bound $\mathrm{SnO}_{2}$ no such recovery of porphyrin emission upon addition of $\mathrm{LiClO}_{4}$ was observed (see Figure 6.12) suggesting much stronger binding of $(\mathrm{TMIP}) \mathrm{H}_{2}$ to the $\mathrm{SnO}_{2}$ surface, a result that is in agreement with the calculated binding constants. This effect can also be seen visually when the (TMPyP)Zn was irradiated by UV light (Figure 6.11 inset). While 


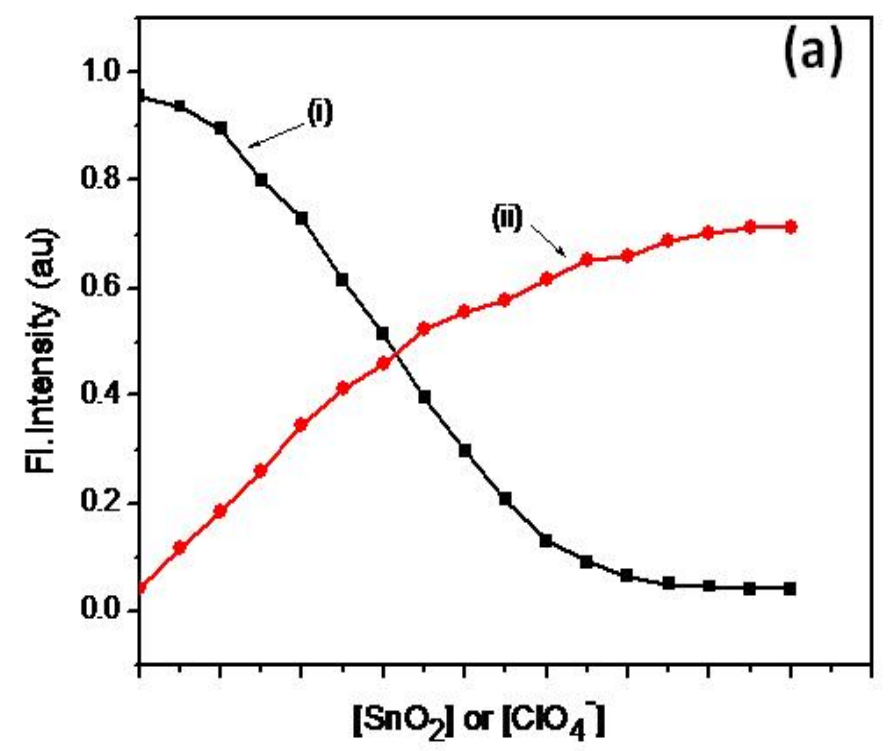

(b)

(i) (ii) (iii)

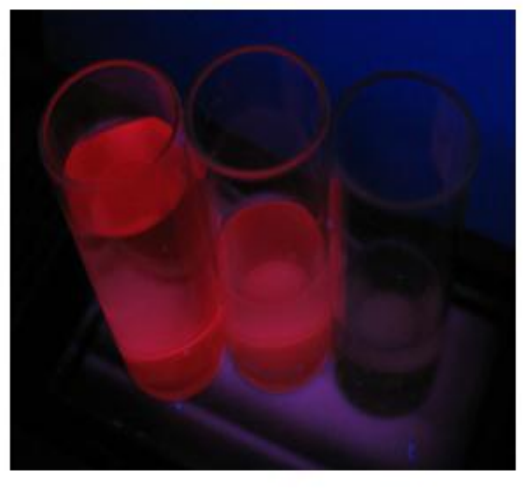

Figure 6.11: (a) (i) Fluorescence intensity of the $660 \mathrm{~nm}$ band of (TMPyP) $\mathrm{Zn}$ on increasing addition of nanocrystalline $\mathrm{SnO}_{2}$ in water (each addition is $15 \mu \mathrm{L}$ of $2.011 \mathrm{~g} / \mathrm{L}$ $\mathrm{SnO}_{2}$ ). (ii) Recovery of porphyrin emission on increasing addition of lithium perchlorate (each addition is $10 \mu \mathrm{L}$ of $1.0 \mathrm{M}$ solution) to a solution of (TMPyP) $\mathrm{Zn}: \mathrm{SnO}_{2}$ in water. (b) The picture shows fluorescence under UV irradiation of (i) (TMPyP)Zn, (ii) $(\mathrm{TMPyP}) \mathrm{Zn}: \mathrm{SnO}_{2}$ in the presence of $\mathrm{LiClO}_{4}$ and (iii) (TMPyP)Zn:SnO 2 complex in water.

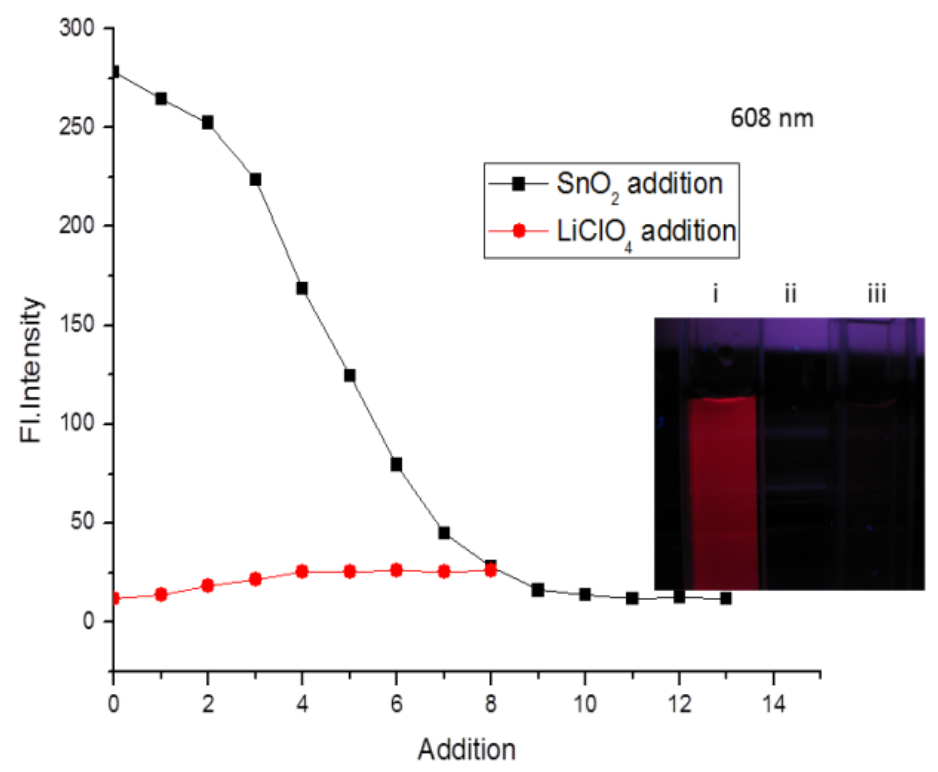

Figure 6.12: Fluorescence intensity of the $660 \mathrm{~nm}$ band of (TMIP) Zn on increasing addition of nanocrystalline $\mathrm{SnO}_{2}$ (dark) and lithium perchlorate (red) to a solution of $(\mathrm{TMIP}) \mathrm{Zn}: \mathrm{SnO}_{2}$ in water. The picture shows fluorescence under UV irradiation of (i) (TMIP)Zn, (ii) (TMIP)Zn:SnO 2 in the presence of $\mathrm{LiClO}_{4}(\sim 100$ fold addition). 
(TMPyP)-Zn:SnO 2 resulted in a dark complex (picture $\mathrm{c}$ in Figure 6.11 inset), restoration of fluorescence upon addition of lithium perchlorate is clearly seen (picture b in Figure 6.11 inset) indicating release of fluorescent porphyrin from the $\mathrm{SnO}_{2}$ surface. It may be mentioned here that utilization of neutral porphyrin with no peripheral positive charges resulted little or no $\mathrm{SnO}_{2}$ surface adsorption.

\subsubsection{Photoelectrochemical Studies}

Photoelectrochemical studies were performed using $\mathrm{SnO}_{2}$ thin-film on the FTO electrode surface after electrostatic adsorption of the positively charged porphyrins. The $\mathrm{SnO}_{2}$ electrodes were dipped in $\sim 1 \mathrm{mM}$ concentration of a given porphyrin solution in methanol and the adsorption was monitored by optical absorption methods. After reaching the maximum adsorption (nearly 3 hours), the electrodes rinsed with pure methanol to remove any unbound porphyrins. They were dried at $60^{\circ} \mathrm{C}$ for $10 \mathrm{~min}$ to remove the solvent and were used in the following studies. The amount of porphyrin on thin film $\mathrm{SnO}_{2}$ surface for (TMPyP)Zn modified electrode, estimated from optical studies (desorption method by the addition of $\mathrm{LiClO}_{4}$ ), was about $3.5 \times 10^{-7} \mathrm{moles} / \mathrm{cm}^{2}$. Figure $6.13 \mathrm{a}$ and $6.13 \mathrm{~b}$ shows the absorption spectra of the adsorbed porphyrins after background subtraction for $\mathrm{FTO} / \mathrm{SnO}_{2}$ along with the picture of the modified electrodes. The electrodes were greenish-red, different from that of red/purple color of the porphyrins in solution indicating not a simple adsorption but a good surface interaction. High absorbance was observed in the Soret region while in the visible region, the normal spectral bands corresponding to either the zinc or free-base porphyrin were apparent. It is important to note the existence of the broadened spectral bands for (TMPyP)M adsorbed electrodes suggesting occurrence of structural changes 
associated with flattening of the pyridyl rings to the plane of the porphyrin macrocycle upon binding to the $\mathrm{SnO}_{2}$ surface, ${ }^{300}$ in agreement with the absorption spectral results shown in Figure 6.1.
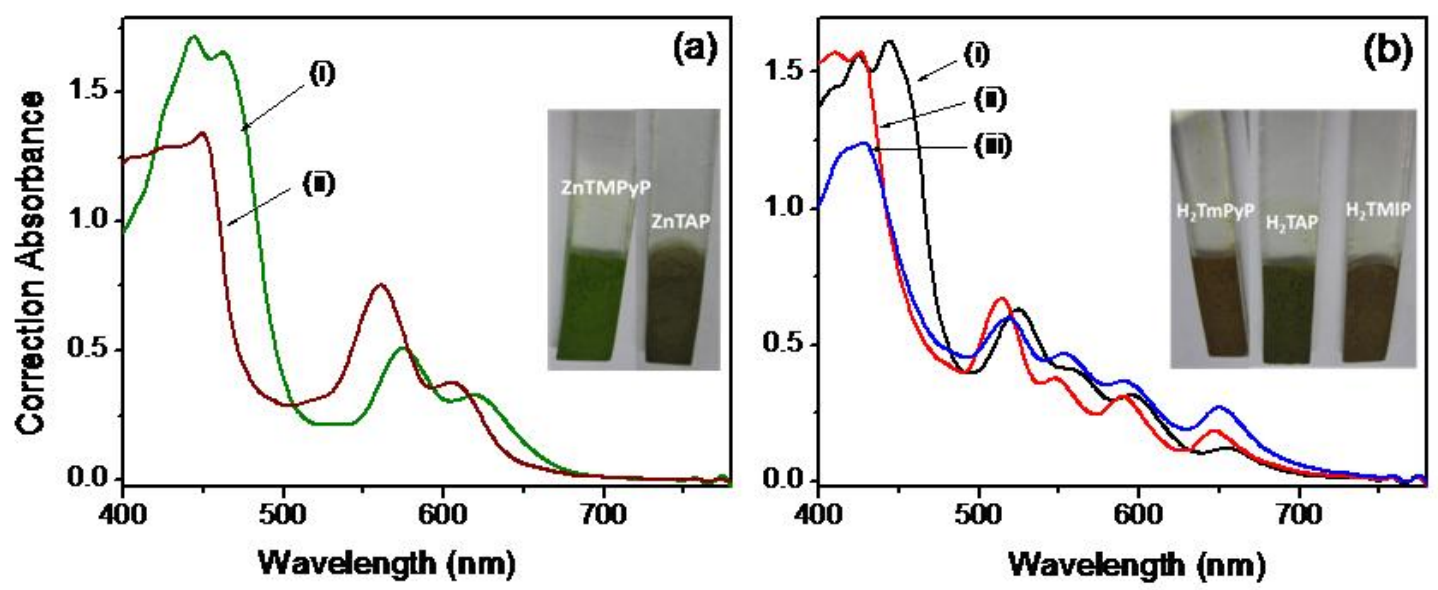

Figure 6.13: Corrected absorbance (background subtracted for $\mathrm{FTO} / \mathrm{SnO}_{2}$ ) spectrum of (a) zinc and (b) free-base derivatives of (i) TMPyP, (ii) TAP and (iii) TMIP derivatives electrostatically adsorbed onto the thin film $\mathrm{SnO}_{2}$ on FTO electrodes. The figure inset shows the picture of the respective $\mathrm{FTO} / \mathrm{SnO}_{2} /(\mathrm{P}) \mathrm{M}$ electrodes.

Figure 6.14 a shows $\mathrm{I}-\mathrm{V}$ characteristics of the $\mathrm{FTO} / \mathrm{SnO}_{2} / \mathrm{M}(\mathrm{P})$ in the presence of $I^{-} / I_{3}^{-}$redox mediator under AM 1.5 simulated light conditions. The choice of iodide salts with regard to the cationic counterpart deserves special mention as stronger interacting cation might replace the electrostatically adsorbed porphyrins on the $\mathrm{SnO}_{2}$ surface. After a series of trials we found that tetrabutylammonium iodide ((TBA)I) had no effect on the electrostatically adsorbed cationic porphyrins on the $\mathrm{SnO}_{2}$ surface. This was confirmed by measuring the absorbance of the mediator $\mathrm{I}^{-} / \mathrm{I}_{3}{ }^{-}$solution before and after the photoelectrochemical experiment which showed no traces of desorbed porphyrin in solution. Hence, (TBA)I was used in the preparation of the mediator $\mathrm{I}^{-} / \mathrm{I}_{3}^{-}$solution. As shown in Figure 6.14b, a steady anodic photocurrent was observed when the $\mathrm{FTO} / \mathrm{SnO}_{2} /(\mathrm{P}) \mathrm{M}$ electrodes were illuminated. The short circuit current, Isc ranged 
between $3.3 \mathrm{~mA} / \mathrm{cm}^{2}$ and $4.7 \mathrm{~mA} / \mathrm{cm}^{2}$ while the open circuit potential, $V_{\mathrm{OC}}$ ranged between 0.13 and $0.24 \mathrm{~V}$ depending upon the nature of porphyrin adsorbed onto the $\mathrm{SnO}_{2}$ surface. All of the modified electrodes revealed some dark currents beyond $0.1 \mathrm{~V}$ (dotted lines in Figure 6.14a), more so for the (TMPyP)M modified electrodes. In the case of (TAP)M and (TMPyP) $\mathrm{H}_{2}$, dark currents were normally lower which reflected in a 50-70 mV increase in $V_{\text {oc. }}$. Such behavior was reported for $\mathrm{SnO}_{2}$ modified with a ruthenium complex, N719, ${ }^{306-308}$ and the effect was attributed to the lower conduction band edge resulting in higher back electron transfer rates ${ }^{309,310}$ and higher reactive trap energy states. ${ }^{306-308}$ Between the free-base and zinc porphyrins, the latter revealed better performance in terms of both $I_{\mathrm{SC}}$ and $V_{\mathrm{OC}}$, a result that could to be related to freeenergy changes and bathochromic shift upon adsorption of the porphyrins. The fillfactors were also calculated from the $J$ - $V$ plots whose values range
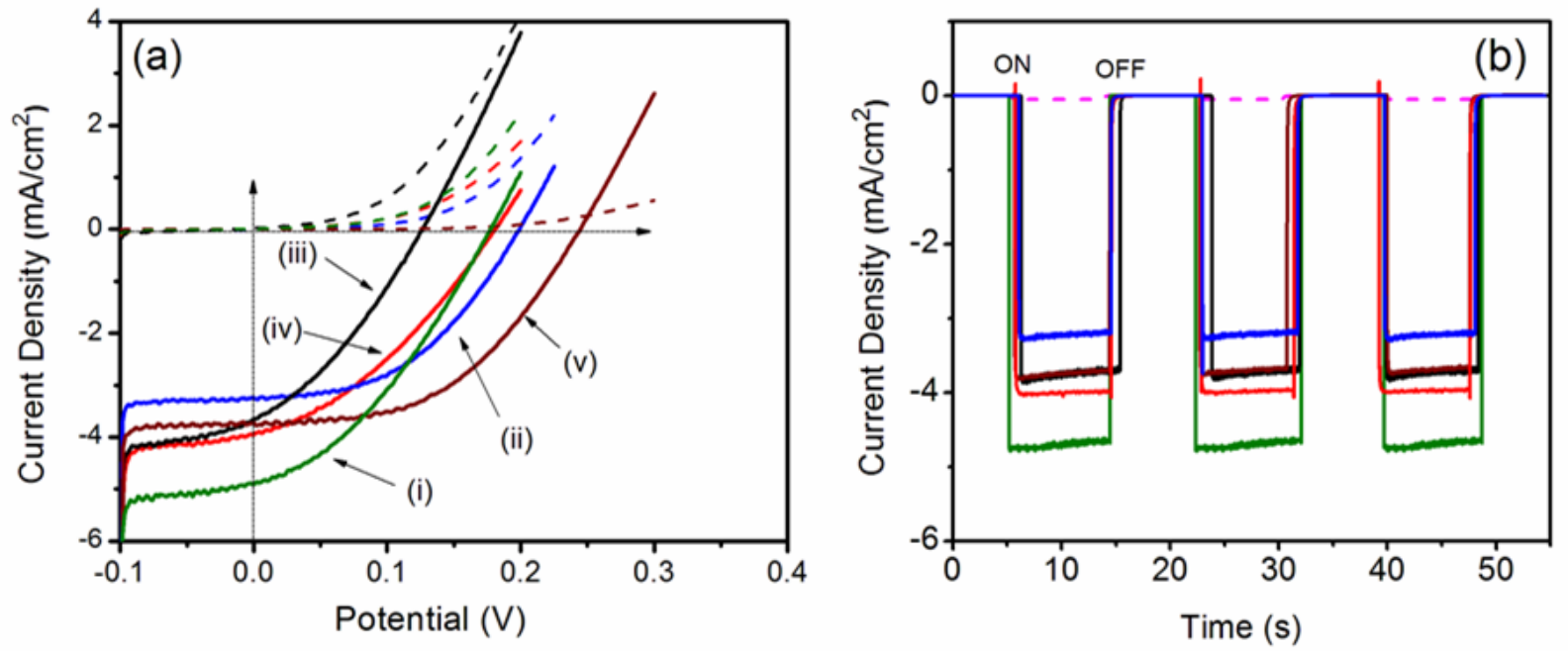

Figure 6.14: (a) $\mathrm{J}-V$ plots for $\mathrm{FTO} / \mathrm{SnO}_{2} /(\mathrm{P}) \mathrm{M}$ electrodes in acetonitrile containing $0.5 \mathrm{M}$ (TBA)I and $0.05 \mathrm{M} \mathrm{I}_{2}$ as redox mediator; (i) (TMPyP)Zn, (ii) (TAP)Zn, (iii) (TMPyP) $\mathrm{H}_{2}$, (iv) (TAP) $\mathrm{H}_{2}$ and (v) (TMIP) $\mathrm{H}_{2}$ surface modified. The dotted lines show the dark currents. Figure (b) shows light on-off switching of photocurrent revealing the robustness of the electrodes. The dotted line represents currents of electrode modified with only $\mathrm{SnO}_{2}$. 
between $20-50 \%$ with generally higher fill-factors for the zinc porphyrin derivatives. As shown Figure 6.14b, the light-switching experiments revealed reproducible results suggesting higher stability of the dye- $\mathrm{SnO}_{2}$ of the photoelectrochemical cells.

The monochromatic incident photon-to-current conversion efficiency (IPCE), defined as the number of electrons generated by light in the outer circuit divided by the number of incident photons, was determined according to Equation (2) ${ }^{149}$

$$
\operatorname{IPCE}(\%)=100 \times 1240 \times I_{\mathrm{sc}}\left(\mathrm{mA} \mathrm{cm}^{-2}\right) /\left[\lambda(\mathrm{nm}) \times P_{\mathrm{in}}\left(\mathrm{mW} \mathrm{cm}^{-2}\right)\right]
$$

where $I_{\mathrm{SC}}$ is the short-circuit photocurrent generated by the incident monochromatic light and $\lambda$ is the wavelength of this light with intensity $P_{\text {in. }}$. The photocurrent action spectrum (average of three runs) of the $\mathrm{FTO} / \mathrm{SnO}_{2} / \mathrm{M}(\mathrm{P})$ modified electrodes in a mediator solution of $0.5 \mathrm{M}(\mathrm{TBA}) \mathrm{I}$ and $0.05 \mathrm{M} \mathrm{I}_{2}$, in acetonitrile, with a Pt foil as the counter electrode, is shown in Figure 6.15. The spectra resembled those of the absorption spectra shown in Figure $6.13 \mathrm{a}$ and $\mathrm{b}$. At the wavelength of maximum photocurrent, the IPCE was $91 \%$ for $\mathrm{FTO} / \mathrm{SnO}_{2} /(\mathrm{TMPyP}) \mathrm{Zn}$ electrode at the Soret band area for $\mathrm{SnO}_{2^{-}}$ dye modified electrodes in a photoelectrochemical setup. Additionally, the observed red shifted peaks of (TMPyP)M is in agreement with the optical absorption spectrum shown in Figure 6.13 as a consequence of flattening of the porphyrin macrocycle on the $\mathrm{SnO}_{2}$ surface. The high IPCE values suggest that in addition to electrostatic interactions, good orbital interactions between (TMPyP)M and conduction band of $\mathrm{SnO}_{2}$ as a consequence of favorable geometry of the macrocycle (flattening of the peripheral aryl substituents to the macrocycle plane) has resulted in better charge injection. ${ }^{312}$ Table 6.2 lists summary of the performance of the solar cells developed in the present study. IPCE values at each peak maxima of porphyrin are listed. 

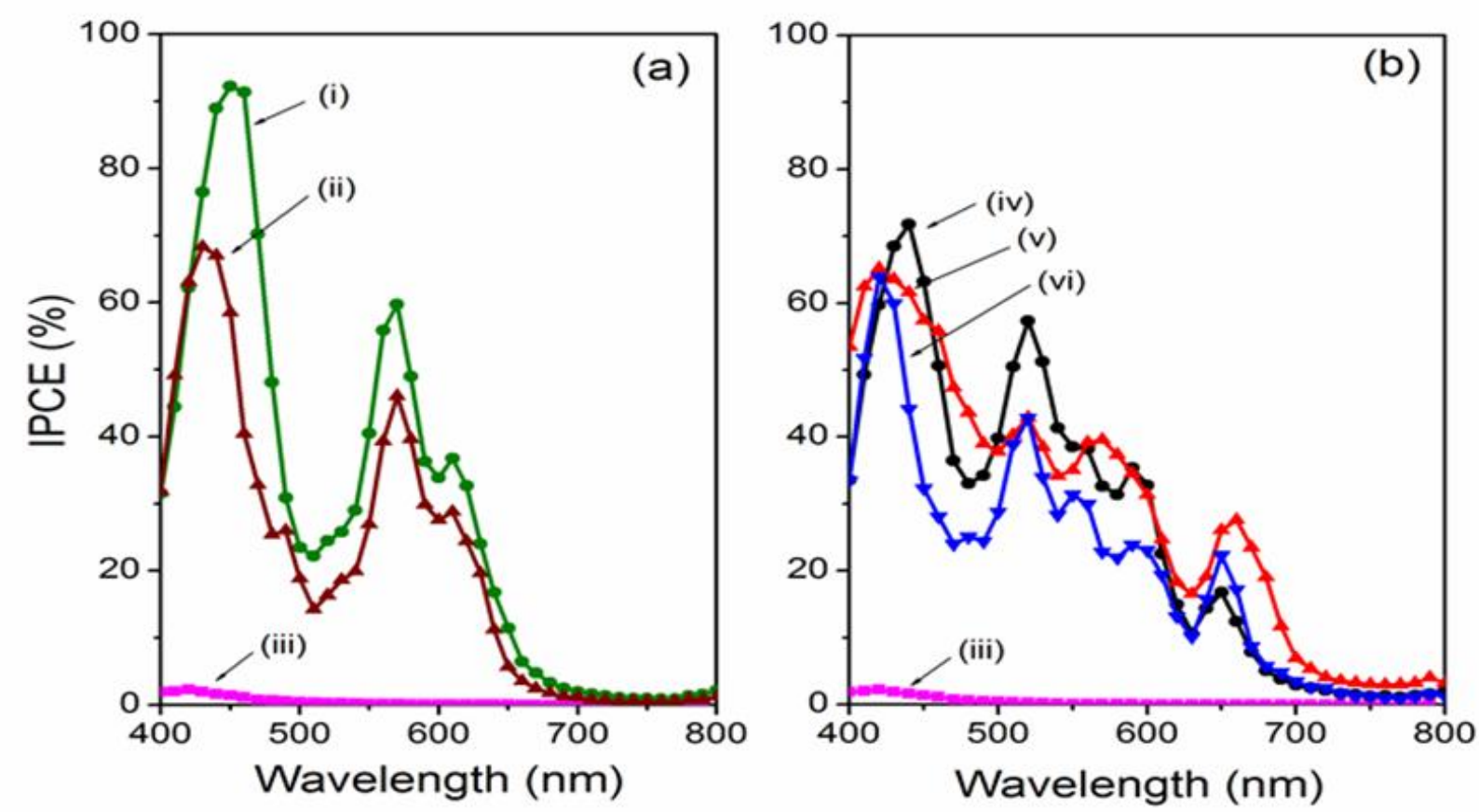

Figure 6.15: Incident photon-to-current conversion efficiency (IPCE) for $\mathrm{FTO} / \mathrm{SnO}_{2} /(\mathrm{P}) \mathrm{M}$ electrodes in acetonitrile containing (TBA) $1 / \mathrm{I}_{2}(0.5 \mathrm{M} / 0.05 \mathrm{M})$ redox mediator. The porphyrins electrostatically adsorbed are: (i) (TMPyP)Zn, (ii) (TAP)Zn, (iii) no porphyrin, (iv) $(\mathrm{TMPyP}) \mathrm{H}_{2}$, (v) (TAP) $\mathrm{H}_{2}$ and (vi) $(\mathrm{TMIP}) \mathrm{H}_{2}$, respectively on $\mathrm{FTO} / \mathrm{SnO}_{2}$ surface.

Table 6.2: Performance of the $\mathrm{FTO} / \mathrm{SnO}_{2} / \mathrm{M}(\mathrm{P})(\mathrm{P}=\mathrm{TMPyP}$, TAP or TMIP) solar cells investigated in the present work.

\begin{tabular}{|c|c|c|c|c|c|}
\hline$\overline{M(P)}$ & Peak position IPCE (\%) & $\begin{array}{c}I_{\mathrm{sc}}, \\
\mathrm{mA} / \mathrm{cm}^{2}\end{array}$ & $V_{\text {OC }}, V$ & $\mathrm{FF}, \%$ & $\eta, \%$ \\
\hline (TMPyP)Zn & $455(91), 570(68), 612(39)$ & 4.7 & 0.18 & 38 & 0.31 \\
\hline$(T A P) Z n$ & $431(58), 570(45), 611(27)$ & 3.7 & 0.24 & 50 & 0.45 \\
\hline (TMPyP) $\mathrm{H}_{2}$ & $\begin{array}{c}439(71), 519(57), 588(38) \\
590(35), 652(16)\end{array}$ & 3.7 & 0.13 & 31 & 0.15 \\
\hline$(\mathrm{TAP}) \mathrm{H}_{2}$ & $\begin{array}{c}420(64), 520(43), 570(38), \\
590(35), 659(27)\end{array}$ & 4.0 & 0.18 & 34 & 0.25 \\
\hline$(\mathrm{TMIP}) \mathrm{H}_{2}$ & $\begin{array}{c}422(63), 519(43), 556(31), \\
596(24), 652(22)\end{array}$ & 3.3 & 0.20 & 38 & 0.31 \\
\hline
\end{tabular}


Integrating the IPCE $(\lambda)$ spectrum over $A M 1.5,100 \mathrm{~mW} / \mathrm{cm}^{2}$ gives the theoretical current density $\left(J_{\mathrm{sc}}\right){ }^{313}$ As shown in the Figure 6.16a, theoretical and practical values were in close agreement with a linear regression $\left(R^{2}\right)$ value of 0.8 for the best fit line. Although very high IPCE values have been obtained, due to lower $V_{\mathrm{OC}}$, an intrinsic property of $\mathrm{SnO}_{2}$ and moderate fill-factors, the overall light energy conversion efficiencies were found to be relatively small ranging between $0.15 \%-0.45 \%$.
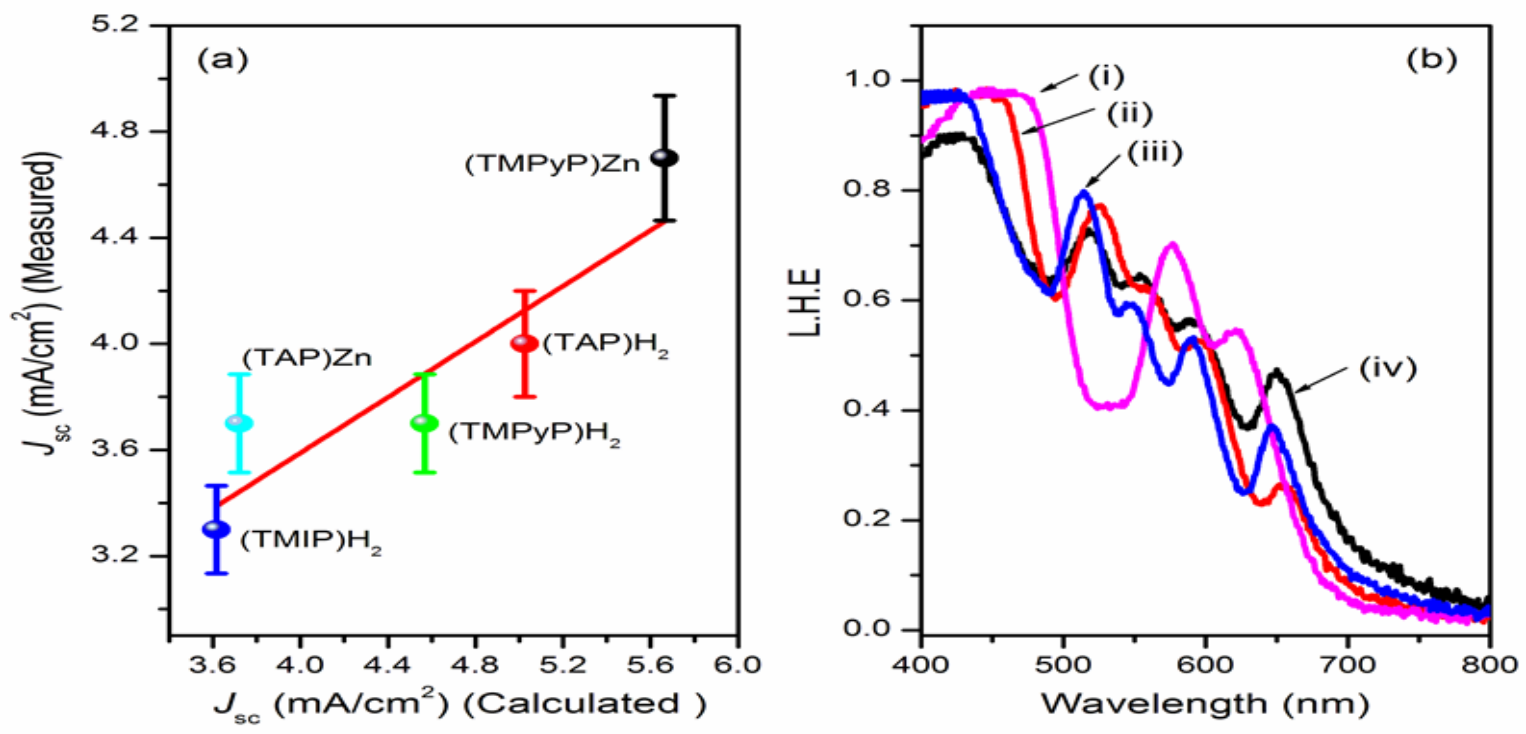

Figure 6.16: (a) Calculated vs. measured $J_{s c}$ for different porphyrin adsorbed electrodes. Theoretical calculation were performed using the formula $J_{s c}=\int q \times F(\lambda) x$ IPCE ( $\lambda) d \lambda$ where $q$ is the electron charge and $F(\lambda)$ is the incident photon flux density for AM 1.5 standard conditions at wavelength ( $\lambda$ ). (b) Light harvesting efficiency of (i) (TMPyP)Zn, (ii) (TMPyP) $\mathrm{H}_{2}$, (iii) (TAP) $\mathrm{H}_{2}$ and (iv) (TMIP) $\mathrm{H}_{2}$ electrodes.

Since IPCE is directly related to the light harvesting efficiency (LHE), the LHE for the present electrodes were also calculated using literature procedure. ${ }^{4}$ As shown in Figure 6.16b, for (TMPyP)Zn modified electrode, the efficiency was maximum (100\%) in the wavelength range of $400-457 \mathrm{~nm}$, however, for other electrodes high LHE values were obtained in this range. In the Q-band range (500-700 $\mathrm{nm}$ range), these values were $20-80 \%$. Such high efficiency might be attributed the very high extinction 
coefficient of porphyrin macrocycle in the Soret region and associated structural changes of the porphyrin macrocycle.

\subsubsection{Electrochemical Impedance Spectroscopic (EIS) Studies}

In order to understand the porphyrin- $\mathrm{SnO}_{2} /$ electrolyte interface, electrochemical impedance studies were performed on representative electrodes modified with (TMPyP)M ( $\mathrm{M}=\mathrm{Zn}$ or $2 \mathrm{H})$. For photoelectrochemical cells, EIS has been a useful tool to estimate electron recombination resistance and to understand the of dye regeneration efficiency. ${ }^{4,314}$ Figure 6.17 shows EIS results along with equivalent circuit diagram used in the analysis. Both electrodes revealed high resistance under dark conditions compared to the values under illumination. The recombination resistance under dark conditions for (TMPyP)Zn was $177.3 \Omega \mathrm{cm}^{2}$ while that for (TMPyP) $\mathrm{H}_{2}$ it was $123.0 \Omega \mathrm{cm}^{2}$ at $V_{\mathrm{oc}}$. Interestingly, under AM1.5 light conditions at $V_{\mathrm{oc}}$, the recombination resistance for (TMPyP) $\mathrm{H}_{2}$ was slightly higher being $76.6 \Omega \mathrm{cm}^{2}$ than that of (TMPyP)Zn being $65.6 \Omega \mathrm{cm}^{2}$. The decrease in the recombination resistance under light can be attributed to increased local concentration of $\mathrm{I}_{3}{ }^{-}$due to the regeneration of dye molecule by iodide near to the dye electrode-interface. ${ }^{4,314}$ That is, photo-regeneration of (TMPyP)Zn is much efficient compared to $(\mathrm{TMPyP}) \mathrm{H}_{2}$, a result that agrees well with the cell efficiency measured by the IPCE curves in Figure 6.15.

Finally, a comparison between the present IPCE values with the literature results on high performance $\mathrm{ITO} / \mathrm{SnO}_{2} /$ dye electrodes deserves special mention. Until this work, the highest IPCE for $\mathrm{SnO}_{2}$ surface modified with a photosensitizer was for a cationic $\mathrm{Ru}(\mathrm{II})$ polypyridyl complex where a maximum IPCE of $20 \%$ at $480 \mathrm{~nm}$ was

reported. ${ }^{88}$ Interestingly, for a few elegant porphyrin-fullerene donor-acceptor dyads 
deposited electrophoretically onto the $\mathrm{SnO}_{2}$ surface, IPCE values up to $60 \%$ have been reported. ${ }^{46,56,149,266,315}$ Clearly, the present results have demonstrated that the electrostatic binding of water soluble cationic porphyrins to nanocrystalline $\mathrm{SnO}_{2}$ surface under appropriate conditions of binding and electronic interactions, serve as a convenient approach to obtain electrodes capable of giving near unity photon-toelectron conversion efficiencies.

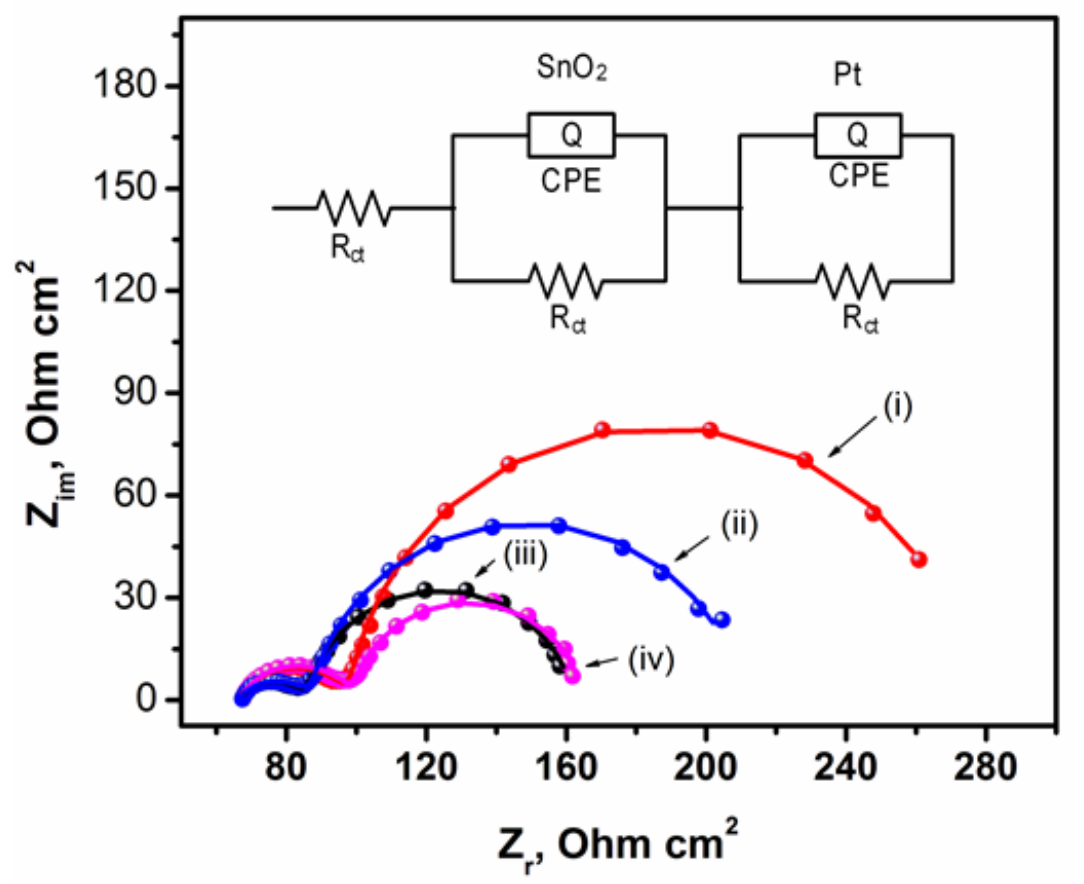

Figure 6.17: Impedance spectra (Nyquist plots) measured at the respective $V_{O C}$ of (TMPyP)Zn and (TMPyP) $\mathrm{H}_{2}$ in dark (i and ii) and under AM1.5 light conditions (iii and iv), respectively. The figure inset shows equivalent circuit diagram used to fit the data.

\subsection{Summary}

A relatively simple approach of electrostatic surface decoration of nanocrystalline thin film $\mathrm{SnO}_{2}$ electrodes by cationic water soluble porphyrins is demonstrated. Freebase and zinc(II) derivatives of three types of cationic water soluble porphyrins having positive charges at distant positions of the macrocycle periphery are utilized. The porphyrin binding to $\mathrm{SnO}_{2}$ porphyrin in water is found to be stable as revealed by their 
binding constants with a 1:1 molar stoichiometry. Both steady-state and time-resolved emission spectra revealed quenching of porphyrin emission upon binding to $\mathrm{SnO}_{2}$ in water suggesting electron injection from singlet excited porphyrin to $\mathrm{SnO}_{2}$ conduction band. Addition of $\mathrm{LiClO}_{4}$ weakened the ion-paired porphyrin- $\mathrm{SnO}_{2}$ binding as revealed by reversible emission changes. Photoelectrochemical studies performed on $\mathrm{FTO} / \mathrm{SnO}_{2} /(\mathrm{P}) \mathrm{M}$ electrodes revealed IPCE values up to $91 \%$ at the peak maxima, reported for $\mathrm{SnO}_{2}$-dye modified electrodes. The high IPCE values have been attributed to improved charge injection due to strong electrostatic and electronic interactions between the (TMPyP)M and $\mathrm{SnO}_{2}$, better light harvesting efficiency of porphyrins and also due to better regeneration as confirmed by EIS. Under AM 1.5 simulated light conditions, the short circuit current, ISc was in the order of 3.3-4.7 A/cm ${ }^{2}$, and the open circuit potential, $V_{\mathrm{OC}}$ was in the amount of $\sim 0.2 \mathrm{~V}$ resulting in a maximum light conversion efficiency of $0.45 \%$. Electrochemical impedance spectroscopy studies revealed overall decreased electron recombination resistance under light illumination conditions, more so for (TMPyP)Zn. These studies show that the decoration of cationic photosensitizers onto the $\mathrm{SnO}_{2}$ nanoparticles under favorable electrostatic and electronic interactions is significant in terms of achieving higher photon-to-electron conversion efficiency, and this strategy can be further exploited to develop high efficiency light energy conversion photocells. 


\section{CHAPTER 7}

\section{ION-PAIRED HETERODIMER SUPRAMOLECULAR SYSTEM}

\subsection{Introduction}

Co-sensitization for solar energy harvesting devices has recently gained interest as it provides new pathways for efficient near-IR absorption sensitized systems ${ }^{316}$. Selection of dyes for co-sensitization is very crucial role for developing such wide band energy harvesting systems. Sensitizing dyes could be chosen based on their 1) absorption properties i.e. should absorb at different portion of wavelength and able to inject electron for photocurrent $\left.{ }^{86,317}: 2\right)$ dyes that can undergo FRET type efficient energy transfer upon immobilized on the surface. In some cases FRET energy donor dyes molecules are added in the electrolyte that could transfer energy to the acceptor facilitating electron injection ${ }^{80,318}$. In all the co sensitization system studied, mostly dyes containing only carboxylic acid have been used as it can be adsorbed on the metal oxide strongly through bridging mode. Sensitizing cocktail solution composed of different of the dye molecules that are used to prepare efficient system. In order to force the energy harvesting to near NIR region, there is a need for intensive design ${ }^{82}$ and synthetic methods for dye molecules to be used for sensitization ${ }^{81,83,319}$. Another approach would be is to self-assemble pre-organized dyes with suitable properties on a metal oxide layer at particular configuration using multiple weak intermolecular interactions ${ }^{320}$. In photosynthetic reaction center, for example, the special pair bacteriochlorophyll dimer molecule ${ }^{321}$ is held together by multiple interactions such as Vander Waals, hydrogen bonding and hydrophobic interaction and serves as the energy harvesting center. 
Recently, supramolecular systems for energy harvesting and storage applications carbon based nanomaterial and metal oxides are becoming attractive ${ }^{322-324}$. Very few supramolecular works for energy conversion ${ }^{63,325}$ and storage have been reported. Positively charged inorganic and organic dyes upon adsorption on tin oxide are known to produce photocurrents. ${ }^{144,326}$ Our group recently reported highest IPCE using cationic water soluble porphyrins adsorbed on tin oxide due to ion pairing effect $^{327}$. In addition, Girault et al., reported photo generation of current using ion paired water soluble porphyrins based heterodimers at the liquid-liquid interface ${ }^{328}$. Quantum dots such as CdS, CdSe, PbS, are being explored for NIR higher efficiency systems ${ }^{84}$. Ion pairing oppositely charged porphyrins is known to form different nanoform structures such as molecular films ${ }^{329}$,nanowires ${ }^{330}$, nanorods ${ }^{331}$ and one dimensional nanoporous structures $^{332}$. Tran-Thi and his coworkers showed detailed solution studies on formation and photochemistry of various heterodimers involving water soluble porphyrin and phthalocyanine. ${ }^{333,334}$ Until now, tetrapyrrole heterodimers for photo energy conversion have not been studied.

Motivation to extend the IPCE to NIR region using supramolecules enabled us to organize heterodimers of porphyrin and phthalocyanine on tin oxide for energy harvesting applications for the first time. Here we report 4 model heterodimers for this purpose and their systematic study of binding events leading to dimer formation and their significance of the interactions using steady state emission. Modification $\mathrm{SnO}_{2}$ nanoparticle using of negatively charged phthalocyanine sulfonic acid $\left(\mathrm{H}_{2} \mathrm{PcS} \& \mathrm{ZnPcS}\right)$ followed by ion pairing of porphyrin $\left(\mathrm{H}_{2} \mathrm{TMPyP} \& \mathrm{ZnTMPyP}\right)$. The structures of the water soluble compound used for the formation of dimer are shown in the scheme 7.1. 

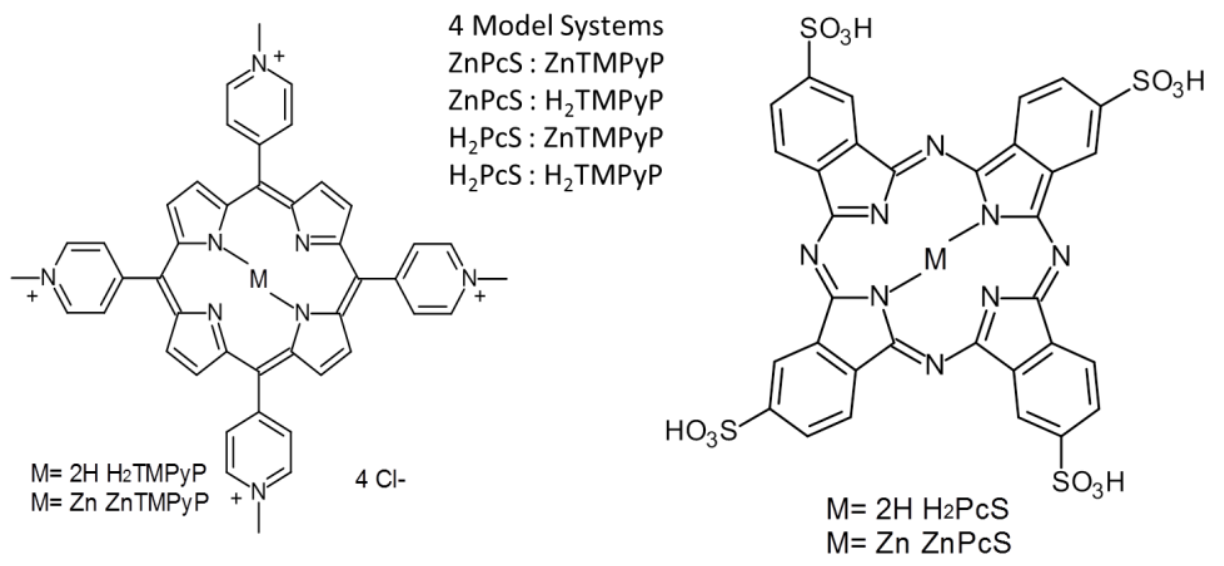

Scheme 7.1: Structures of the cationic water soluble porphyrins and anionic phthalocyanine sulfonic acids employed to surface modify nanocrystalline $\mathrm{SnO}_{2}$ for photoelectrochemical studies in the present study.

7.2 Results and Discussion

\subsubsection{Optical Studies}

Figure 7.1a shows the UV absorption of the entire compound utilized in this study measured in methanol water solution (water - $40 \%$ by volume). Since both of these tetrapyrrole macrocyles have absorption band at different region of visible light, they could be used as the novel preorganized molecules to harvest energy. Cationic water soluble porphyrin derivatives, ZnTMPyP and $\mathrm{H}_{2}$ TMPyP have Soret and $\mathrm{Q}$ band absorbance at $437 \mathrm{~nm}, 421 \mathrm{~nm}$, and $562 \mathrm{~nm}, 591 \mathrm{~nm}, 517 \mathrm{~nm}, 554 \mathrm{~nm}$ and $590 \mathrm{~nm}$ respectively. Change in the orbital symmetry from $d_{2} h$ to $d_{4} h$ upon metal insertion resulted in red shift of the bands in ZnTMPyP compared to $\mathrm{H}_{2}$ TMPyP. Similarly, anionic phthalocyanine derivatives, $\mathrm{H}_{2} \mathrm{PcS}$ and $\mathrm{ZnPcS}$ have absorption near $700 \mathrm{~nm}$. The titration of tetra anionic $\mathrm{ZnPcS}$ with tetra cationic $\mathrm{ZnTMPyP}$ results in formation of dimer is showed in the Figure $7.1 \mathrm{~b}$. Incremental addition of ZnPcS to ZnTMPyP redshifts the Soret and and $\mathrm{Q}$ bands of porphyrin around 7-8 $\mathrm{nm}$ with occurrence of clear isobestic point at $453 \mathrm{~nm}$ and $405 \mathrm{~nm}$. Redshift in the pthalocyanine band from $668 \mathrm{~nm}$ to $705 \mathrm{~nm}$ 
is also being observed. Using the difference in absorbance, a mole ratio plot revealed dimer formation of 1:1 as in Figure 7.1c. Similar kind of redshift and appearance of new band for co-facial dimer, isobestic point revealing single binding event and 1: 1 dimer formation was observed all other three systems as shown in Figure 7.2 and 7.3. The degree of red shift of phthalocyanine band in case of $\mathrm{H}_{2} \mathrm{TMPyP}-\mathrm{H}_{2} \mathrm{PcS}$ titration is from $688 \mathrm{~nm}$ to $750 \mathrm{~nm}$. Our results agree with the reported heterodimer formation events for various anionic phthalocyanine and cationic porphyrin by TranThi et al ${ }^{334}$.

Binding constant for the formation of heterodimer is calculated from the difference in absorbance data using Benesi-Hildebrandt ${ }^{335}$ plot as shown in the inset plot of Figure 7.1b. Using the slope and intercept the apparent association constant $K_{a}$ values are calculated to be $4.5 \times 10^{5} \mathrm{M}^{-1}$. Similar $K_{a}$ values are calculated for all the model system and the found $K_{a}$ values follow the trend: ZnTMPyP:ZnPcS > ZnTMPyP: $\mathrm{H}_{2} \mathrm{PcS}>\mathrm{H}_{2}$ TMPyP:ZnPcS > $\mathrm{H}_{2}$ TMPyP: $\mathrm{H}_{2} \mathrm{PcS}$. Various factors that could affect $K_{a}$ values in heterodimer formation include, geometry, solvent donating power, axial ligating ability of the metal atom, relative position of oppositely charged substituent $^{333,336}$. Axial ligation could be omitted due to high polar nature of methanol: water solvent. So the difference in binding values could be mainly attributed to the geometry of the molecules. Steady state emission data are collected simultaneously with absorption studies. As shown in the Figure 7.1d. ZnTMPyP showed emission at $631 \mathrm{~nm}$ upon exciting at its Soret band $437 \mathrm{~nm}$. Upon addition of ZnPcS, emission of porphyrin is quenched almost $100 \%$ and appearance of new band around $800 \mathrm{~nm}$ with an iso-emissive point around $750 \mathrm{~nm}$. Visual evidence of the quenching event is shown in the Figure $7.1 \mathrm{~d}$ inset. 

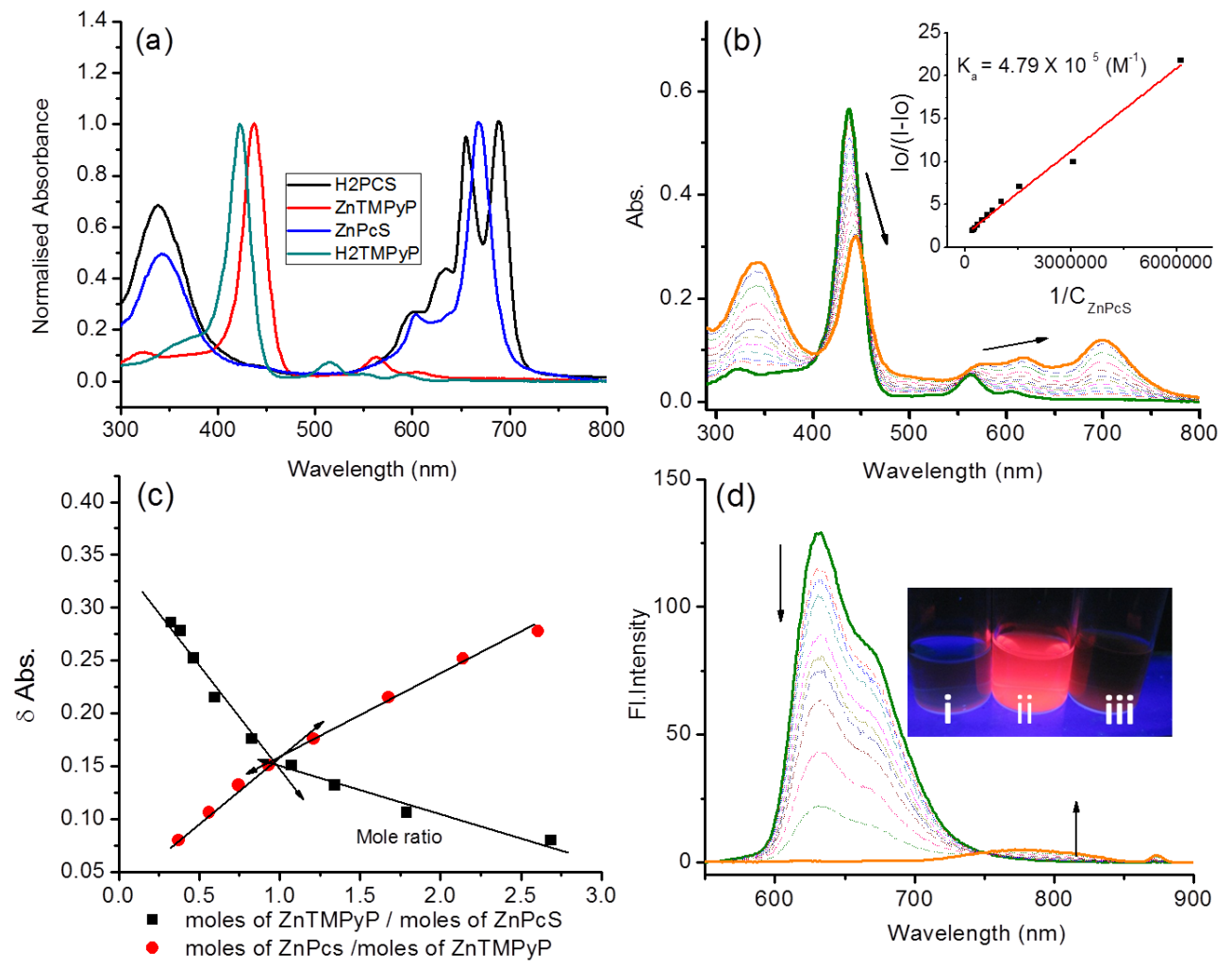

Figure 7.1: (a) Absorption spectra of the compound in methanol-water solvent $\left(\mathrm{H}_{2} \mathrm{O}\right.$ $40 \% \mathrm{Vol})$. (b) Absorption spectral changes observed for ZnTMPyP $(3 \mathrm{ml}$ of $1.75 \mu \mathrm{M})$ on increasing addition $\mathrm{ZnPcS}(5-10 \mu \mathrm{L}$ of $0.489 \mathrm{mM}$ each addition) in methanol-water. (c) Mole ratio vs $\delta A$ at $430 \mathrm{~nm}$ to obtain 1:1 hetero dimer. (d) Corresponding fluorescent spectral changes observed for ZnTMPyP $(3 \mathrm{ml}$ of $\mu \mathrm{M})$ on increasing addition ZnPcS (5$10 \mu \mathrm{L}$ of $0.489 \mathrm{mM}$ each addition) in methanol-water excited at $430 \mathrm{~nm}$. The picture shows fluorescence under UV irradiation of (i) ZnTMPyP, (ii) ZnTMPyP:ZnPcS, (iii) $\mathrm{ZnPcS}$ in methanol-water solvent $\left(\mathrm{H}_{2} \mathrm{O}-40 \% \mathrm{Vol}\right)$. Figure $7.1 \mathrm{~b}$ inset shows BenesiHildebrand plot showing linear dependence of the inverse $\delta A$ at $430 \mathrm{~nm}$ on the inverse of the $\mathrm{ZnPcS}$ concentration.

To confirm the electron transfer event between ZnTMPyP - ZnPcS, upon on addition more than equivalent amount of benzene sulfonic acid, there was no quenching in fluorescence. Very fast excited state electron transfer occurring event has been reported for ZnTMPyP:AIPcS by TranThi in both solution and Langmuir film ${ }^{25}$. The electron transfer in singlet and triplet states resulted in oxidized porphyrin and reduced phthalocyanine as revealed by the transient absorption techniques. We speculate 
similar ligand to ligand charge transfer occurrence as the primary reason for quenching during the ion pair event. The reverse titration of adding ZnTMPyP to ZnPcS also showed quenching revealing the dimer formation. All the model systems showed similar kind of quenching emphasizing photo induced electron transfer event upon heterodimer formation (see Figure $7.2 \& 7.3$ ).
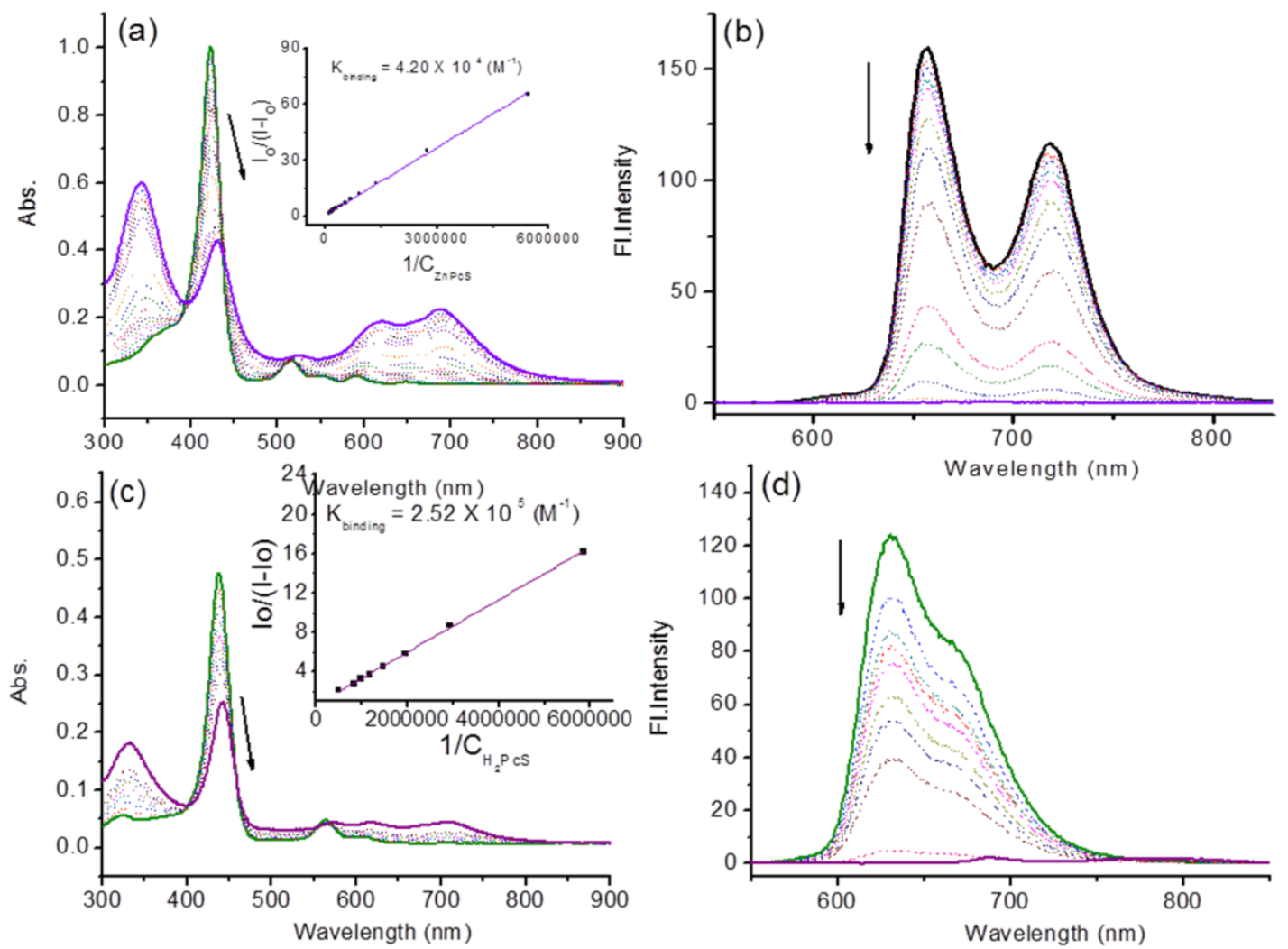

Figure 7.2: (a) Absorption spectral changes observed for $\mathrm{H}_{2}$ (TMPyP) ( $3 \mathrm{ml}$ of $1.45 \mu \mathrm{M}$ ) on increasing addition $\mathrm{Zn}(\mathrm{PcS})(5-10 \mu \mathrm{L}$ of $0.512 \mathrm{mM}$ each addition) in methanol-water. (b) Corresponding fluorescent spectral changes observed for $\mathrm{H}_{2} \mathrm{TMPyP}$ ( $3 \mathrm{ml}$ of 1.45 $\mu \mathrm{M})$ on increasing addition $\mathrm{Zn}(\mathrm{PcS})(5-10 \mu \mathrm{L}$ of $0.512 \mathrm{mM}$ each addition) in methanolwater excited at $438 \mathrm{~nm}$ Figure 1a inset shows Benesi-Hildebrand plot showing linear dependence of the inverse $\delta A$ at $438 \mathrm{~nm}$ on the inverse of the $\mathrm{Zn}(\mathrm{PcS})$ concentration.

(c) Absorption spectral changes observed for $\mathrm{Zn}(\mathrm{TMPyP})(3 \mathrm{ml}$ of $1.75 \mu \mathrm{M})$ on increasing addition $\mathrm{H}_{2}(\mathrm{PcS}) \quad(5-10 \mu \mathrm{L}$ of $0.551 \mathrm{mM}$ each addition) in methanol-water. (d) Corresponding fluorescent spectral changes observed for $\mathrm{Zn}(\mathrm{TMPyP})(3 \mathrm{ml}$ of $1.75 \mu \mathrm{M})$ on increasing addition $\mathrm{H}_{2}(\mathrm{PcS}) \quad(5-10 \mu \mathrm{L}$ of $0.551 \mathrm{mM}$ each addition) in methanol-water excited at $438 \mathrm{~nm}$ Figure 1d inset shows Benesi-Hildebrand plot showing linear dependence of the inverse $\delta A$ at $438 \mathrm{~nm}$ on the inverse of the $\mathrm{H}_{2}(\mathrm{PcS})$ concentration. 

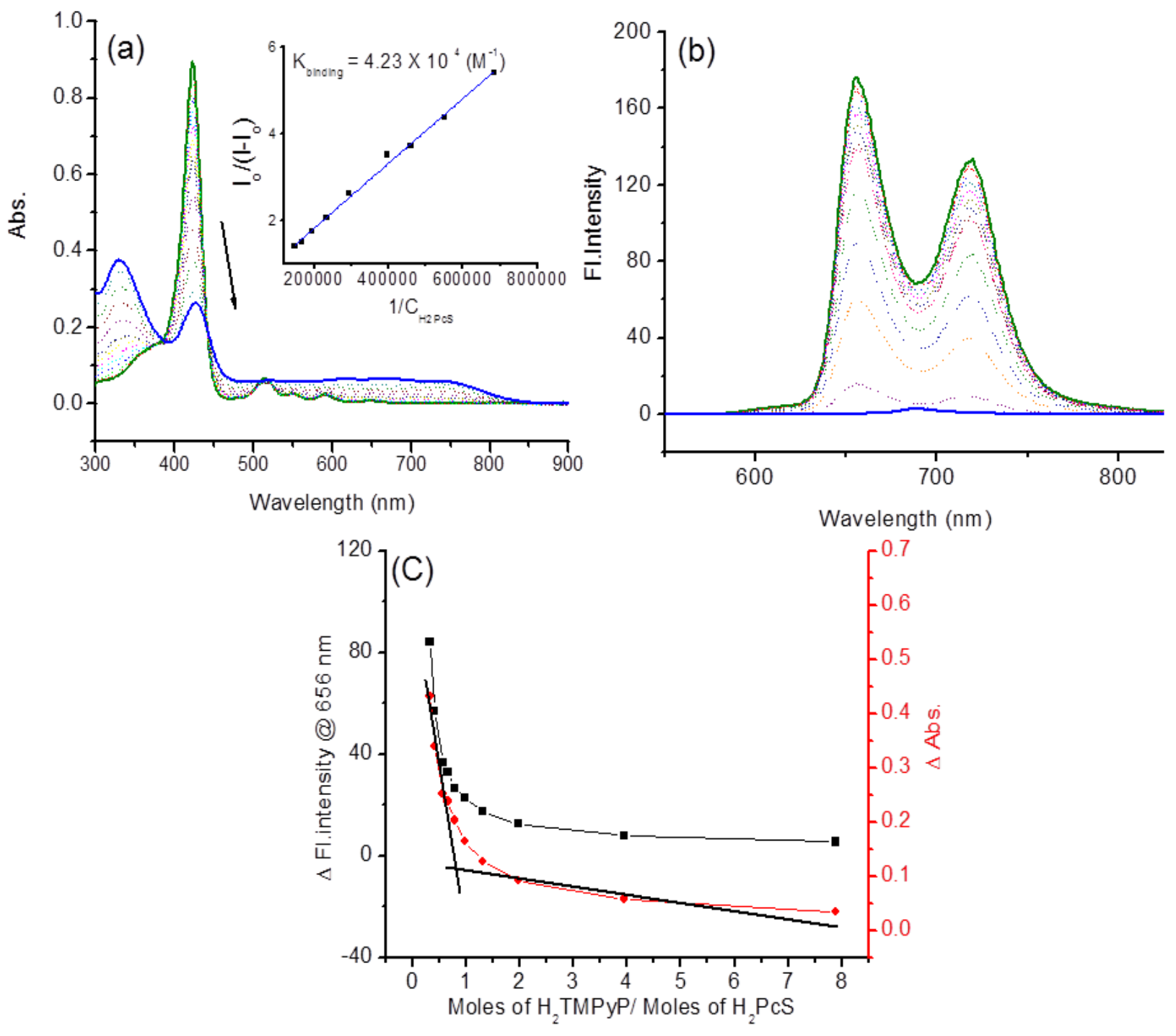

Figure 7.3: (a) Absorption spectral changes observed for $\mathrm{H}_{2}$ TMPyP $(3 \mathrm{ml}$ of $1.45 \mu \mathrm{M})$ on increasing addition $\mathrm{H}_{2}(\mathrm{PcS})(5-10 \mu \mathrm{L}$ of $0.551 \mathrm{mM}$ each addition) in methanol-water. (b) Corresponding fluorescent spectral changes observed for $\mathrm{H}_{2}(\mathrm{TMPyP})$ ( $3 \mathrm{ml}$ of 1.45 $\mu \mathrm{M})$ on increasing addition $\mathrm{H}_{2}(\mathrm{PcS})(5-10 \mu \mathrm{L}$ of $0.551 \mathrm{mM}$ each addition) in methanolwater excited at $438 \mathrm{~nm}$ Figure 1a inset shows Benesi-Hildebrand plot showing linear dependence of the inverse $\delta \mathrm{A}$ at $423 \mathrm{~nm}$ on the inverse of the $\mathrm{H}_{2}(\mathrm{PcS})$ concentration.

\subsubsection{Photoelectrochemistry}

Photoelectrochemical experiments of the heterodimers were performed on nanocrystalline $\mathrm{SnO}_{2}$ layer. Normalized absorbance of the electrodes $\left(\mathrm{ZnPcS} / \mathrm{SnO}_{2}\right.$ and $\mathrm{ZnPcS}-\mathrm{ZnTMPyP} / \mathrm{SnO}_{2}$ ) after adsorption of the dyes was shown in the Figure $7.4 \mathrm{a}$. Inset of the Figure $7.4 \mathrm{a}$ is the actual image of the electrode that was used in 
photoelectrochemical measurement. Adsorption of phthalocyanine sulfonic acid on tin oxide is expected to be in similar to adsorption on titanium dioxide. The absorbance of the Soret band of $\mathrm{ZnPcS}$ broadened upon adsorption on tin oxide. Also the surface coverage of the dye molecules over a period of 90 min dipping was found to be in range of $1.08 \times 10^{-8} \mathrm{~mol} / \mathrm{cm}^{2}$.

The peak broadening was observed due to the interaction of pthalocyanine sulfonic acid with $\mathrm{SnO}_{2}$. Upon immersing the electrode in the cationic porphyrin solutions, instantaneous change of blue color to green was observed. Solution absorbance of the porphyrin solution revealed no phthalocyanine sulfonic acid peak and also no precipitate seen at the bottom due to trimer formation. Absorbance of the ZnTMPyP and ZnPcS modified electrodes showed redshift as reported above in the UV titration. Heterodimer of $\mathrm{H}_{2}$ TMPyP: $\mathrm{H}_{2} \mathrm{PcS}$ showed a broad absorbance upto $800 \mathrm{~nm}$ and ZnTMPyP:ZnPcS showed a stronger interaction as indicated by decrease in the absorbance of $\mathrm{ZnPcS}$. Overall, effective formation of heterodimer on $\mathrm{SnO}_{2}$ was successful and performed the photoelectrochemical experiments on these electrodes.

Figure $7.4 \mathrm{~b}$ showed the photocurrent switching experiment results for $\mathrm{ZnTMPyP} / \mathrm{SnO}_{2}, \mathrm{ZnPcS} / \mathrm{SnO}_{2}$ and $\mathrm{ZnTMPyP}: \mathrm{ZnPcS} / \mathrm{SnO}_{2}$. The heterodimer sensitized electrode photogenerated $\sim 7.1 \mathrm{~mA} / \mathrm{cm}^{2}$ of current density compared to $\mathrm{ZnPcS} / \mathrm{SnO}_{2}$ and $\mathrm{ZnTMPyP} / \mathrm{SnO}_{2}$ electrodes with $3.2 \mathrm{~mA} / \mathrm{cm}^{2}$ and $4.5 \mathrm{~mA} / \mathrm{cm}^{2}$ respectively. So the formation of dimer indeed helped significantly to improve the performance due to better electron transfer as confirmed in the fluorescence solution studies. Of all 4 systems, ZnPcS:ZnTMPyP sensitized $\mathrm{SnO}_{2}$ showed the highest increment due to dimer formation under similar conditions as shown in the Table 7.1. The same system had the highest 
$\mathrm{K}_{\mathrm{a}}$ values from the previous UV absorption studies. So the stronger the binding constant, the higher the photocurrent for a given heterodimers system. These results also agree with our previous system based on axial coordination ${ }^{167}$.
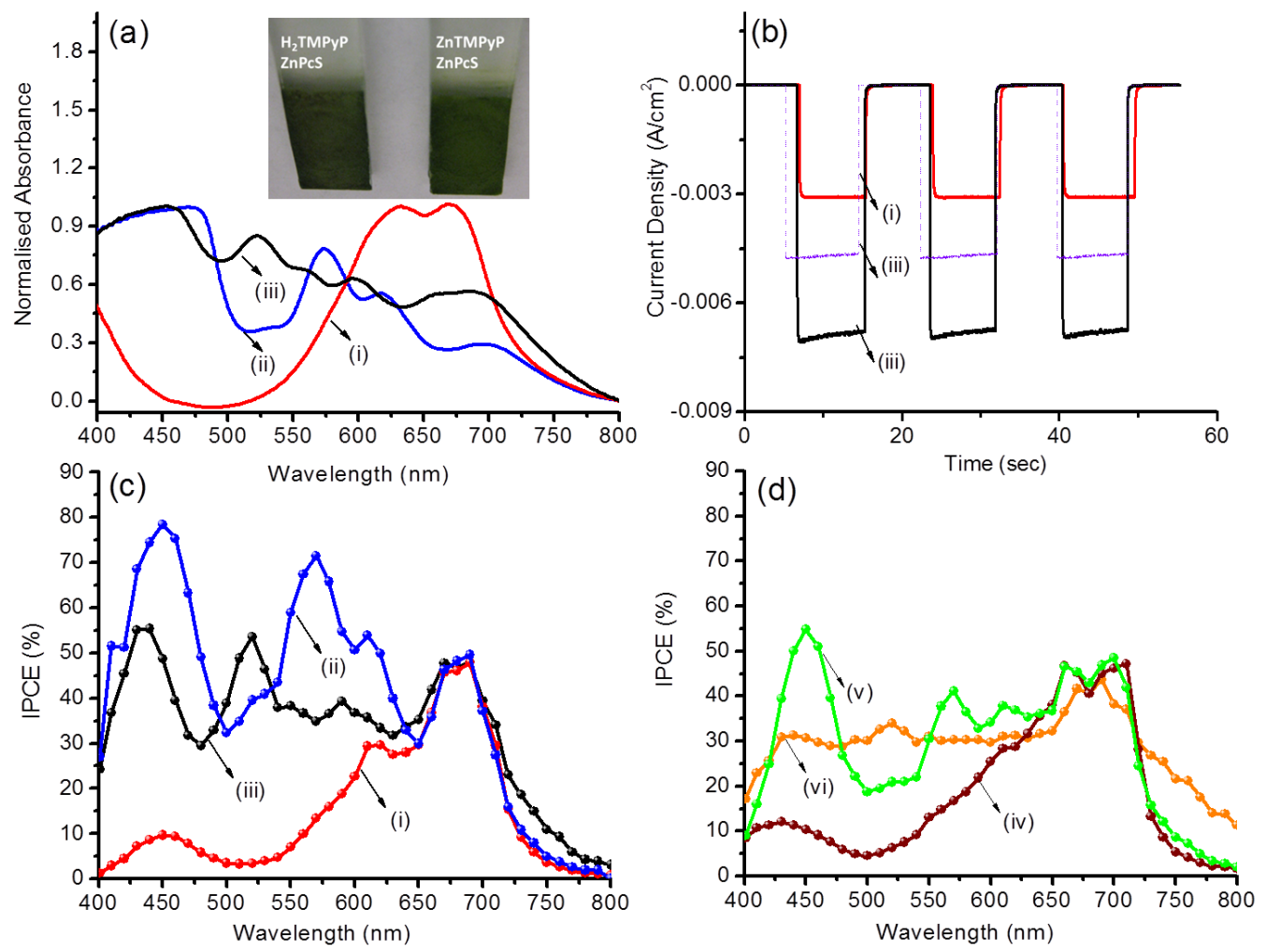

Figure 7.4: (a) Corrected normalized absorbance (background subtracted for $\mathrm{FTO} / \mathrm{SnO}_{2}$ ) spectrum of (i) $\mathrm{ZnPcS} / \mathrm{SnO}_{2}$ (ii) $\mathrm{ZnTMPyP}: \mathrm{ZnPcS} / \mathrm{SnO}_{2}$ and (iii) $\mathrm{H}_{2} \mathrm{TMPyP}$ : $\mathrm{ZnPcS} / \mathrm{SnO}_{2}$. The Figure inset shows the picture of the respective electrodes. (b) shows light on-off switching of photocurrent electrodes i) $\mathrm{ZnPcS} / \mathrm{SnO}_{2}$ (ii) $\mathrm{ZnTMPyP}$ : $\mathrm{ZnPcS} / \mathrm{SnO}_{2}$ and(iii) $\mathrm{ZnTMPyP} / \mathrm{SnO}_{2}$. (c) Incident photon-to-current conversion efficiency (IPCE) for (i) $\mathrm{ZnPcS} / \mathrm{SnO}_{2}$ (ii) $\mathrm{ZnTMPyP}: \mathrm{ZnPcS} / \mathrm{SnO}_{2}$ and (iii) $\mathrm{H}_{2} \mathrm{TMPyP}$ : $\mathrm{ZnPcS} / \mathrm{SnO}_{2}$. (d) IPCE for (iv) $\mathrm{H}_{2} \mathrm{PcS} / \mathrm{SnO}_{2}$ (v) $\mathrm{ZnTMPyP}: \mathrm{H}_{2} \mathrm{PcS} / \mathrm{SnO}_{2}$ and (vi) $\mathrm{H}_{2} \mathrm{TMPyP}: \mathrm{H}_{2} \mathrm{PcS} / \mathrm{SnO}_{2}$. All photoelectrochemical experiments were performed in acetonitrile containing $0.5 \mathrm{M}(\mathrm{TBA}) \mathrm{I}$ and $0.05 \mathrm{M} \mathrm{I}_{2}$ as redox mediator.

Figure 7.4c and Figure 7.4d are the Incident Photocurrent Conversion Efficiency (IPCE) spectra for all 4 model dimer sensitized $\mathrm{SnO}_{2}$ with the corresponding control compounds. IPCE around $50 \%$ was observed for both $\mathrm{ZnPcS} / \mathrm{SnO}_{2}$ and $\mathrm{H}_{2} \mathrm{PCS} / \mathrm{SnO}_{2}$ in their Soret band (650 nm to $700 \mathrm{~nm}) .10 \%$ IPCE for $\mathrm{ZnPcS} / \mathrm{SnO}_{2}$ near $450 \mathrm{~nm}$ jumped to $80 \%$ when ZnTMPyP was ion paired to form the dimer. Panchromatic display of IPCE 
values by the dimer coated electrode (> 50\%) observed from 400 to $700 \mathrm{~nm}$ is shown in the Figure $7.4 \mathrm{c}$ and $7.4 \mathrm{~d}$. The shape of the IPCE curve looks very similar the absorbance of the dimer in solution revealing the dimer formation as the primary reason for their photoelectrochemical behavior.

I-V characteristics for the highest IPCE system ZnTMPyP: $\mathrm{ZnPcS} / \mathrm{SnO}_{2}$ was shown in the Figure7.5a and other electrodes in Figure 7.5b. The efficiency of the electrodes was listed in the Table 7.1. I-V of $\mathrm{ZnPcS} / \mathrm{SnO}_{2}$ alone showed energy efficiency of $0.19 \%$ with short circuit current density $\left(J_{s c}\right)$ and open circuit potential $\left(V_{o c}\right)$ at $3.1 \mathrm{~mA} / \mathrm{cm}^{2}$ and $0.179 \mathrm{~V}$ respectively. Formation of heterodimer ZnTMPyP:ZnPcS/SnO 2 increased energy efficiency $(\eta), V_{o c}$ and $J_{s c}$ to $0.5 \%, 0.212 \mathrm{~V}$ and $7.1 \mathrm{~mA} / \mathrm{cm}^{2}$ respectively. Lower $V_{o c}$ and fill factor could be attributed to low efficiency irrespective of very high IPCE. The more positive positions of conduction band of $\mathrm{SnO}_{2}\left(200 \mathrm{mV}\right.$ more positive compared to $\mathrm{TiO}_{2}$ ) and higher recombination rate could be the reasons for lower $V_{o c}{ }^{304}$.
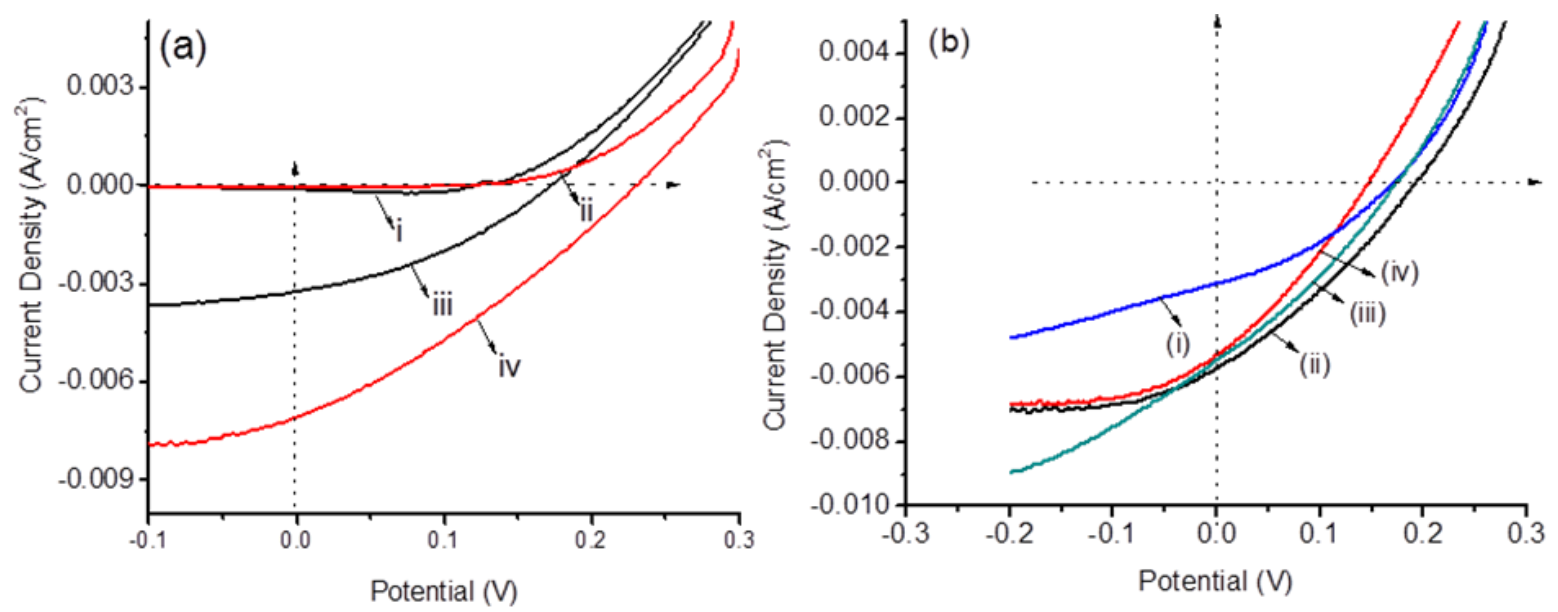

Figure 7.5: (a) $\mathrm{J}-\mathrm{V}$ plots of $\mathrm{ZnPcS} / \mathrm{SnO}_{2}$ and $\mathrm{ZnTMPyP}: \mathrm{ZnPcS} / \mathrm{SnO} \mathrm{O}_{2}$ electrodes in dark (i and ii) and under AM1.5 light conditions (iii and iv), respectively.(b) $\mathrm{J}$ - $V$ plots of (iii) $\mathrm{SnO}_{2} / \mathrm{H}_{2}(\mathrm{PcS})$ (i), $\mathrm{SnO}_{2} / \mathrm{Zn}(\mathrm{PcS}): \mathrm{H}_{2}$ (TMPyP) (ii), $\mathrm{SnO}_{2} / \mathrm{H}_{2}(\mathrm{PcS}): \mathrm{H}_{2}$ (TMPyP) (iii), and $\mathrm{SnO}_{2} / \mathrm{H}_{2}(\mathrm{PcS}): \mathrm{Zn}(\mathrm{TMPyP})$ under $\mathrm{AM} 1.5$ light conditions. The electrolyte used in this experiment consists of $0.5 \mathrm{M}(\mathrm{TBA}) \mathrm{I}$ and $0.05 \mathrm{M} \mathrm{I}_{2}$ as redox mediator. 
Table 7.1: Performance of the $\mathrm{FTO} / \mathrm{SnO}_{2} / \mathrm{M}(\mathrm{P})$ supramolecular solar cells investigated in the present study.

\begin{tabular}{|l|cccccc|}
\hline \multicolumn{1}{|c|}{$\mathrm{M}(\mathrm{P})$} & $K_{\mathrm{a}} 10^{5} \mathrm{M}^{-1}$ & $\mathrm{IPCE}(\%)$ & $\mathrm{J}_{\mathrm{SC}} \mathrm{mA} / \mathrm{cm}^{2}$ & $V_{\mathrm{OC}}, \mathrm{V}$ & $F F \%$ & $\eta \%$ \\
\hline $\mathrm{Zn}(\mathrm{TMPyP})$ & -- & $455(91)$ & 4.7 & 0.18 & 38 & 0.31 \\
$\mathrm{H}_{2}$ (TMPyP) & -- & $439(71)$ & 3.7 & 0.13 & 31 & 0.15 \\
\hline $\mathrm{Zn}(\mathrm{PcS})$ & -- & $688(51)$ & 3.2 & 0.18 & 34 & 0.19 \\
$\mathrm{H}_{2}(\mathrm{PcS})$ & -- & $698(47)$ & 3.1 & 0.17 & 33 & 0.17 \\
\hline $\mathrm{Zn}(\mathrm{PcS}): \mathrm{Zn}(\mathrm{TMPyP})$ & 4.8 & $451(79)$ & 7.0 & 0.19 & 31 & 0.50 \\
$\mathrm{Zn}(\mathrm{PcS}): \mathrm{H}_{2}$ (TMPyP) & 2.5 & $437(55)$ & 5.7 & 0.19 & 31 & 0.34 \\
\hline $\mathrm{H}_{2}(\mathrm{PcS}): \mathrm{Zn}(\mathrm{TMPyP})$ & 0.42 & $449(55)$ & 5.4 & 0.15 & 29 & 0.23 \\
$\mathrm{H}_{2}(\mathrm{PcS}): \mathrm{H}_{2}$ (TMPyP) & 0.41 & $690(44)$ & 5.4 & 0.17 & 31 & 0.29 \\
\hline
\end{tabular}

\subsubsection{Electrochemical Impedance Spectroscopy}

To understand $\mathrm{FTO} / \mathrm{SnO}_{2} /$ heterodimer/electrolyte interface Nyquist plot was recorded at the $V_{o c}$ under dark and light as shown in the Figure7.6. The equivalent circuit used to fit the data to extract the parameter was shown in the Figure7.6 inset.

There are typically 3 arcs in the impedance spectra photoelectrochemical cells ${ }^{314}-$ i) The high frequency region $(500 \mathrm{KHz}-1000 \mathrm{~Hz}$ ) corresponding to charge transport process occurring at the electrolyte/Pt interface: ii) the mid frequency $(1000 \mathrm{~Hz}-1 \mathrm{~Hz})$ region related to charge transport process at the $\mathrm{SnO}_{2} /$ heterodimer/electrolyte interface: iii) the low frequency $(1 \mathrm{~Hz}-0.002 \mathrm{~Hz})$ region represents the mass transport resistance of $\mathrm{I}_{3}{ }^{-}$ion in the electrolyte. As shown in the Figure7.6 due to instrumentation our impedance spectra are limited to only two regions high and mid frequency regions. The recombination resistance $(R c t)$ of the $\mathrm{ZnPcS} / \mathrm{SnO}_{2}$ electrode at $V_{o c}$ in dark and light was found to be $100 \Omega \mathrm{cm}^{2}$ and $82.73 \Omega \mathrm{cm}^{2}$. 


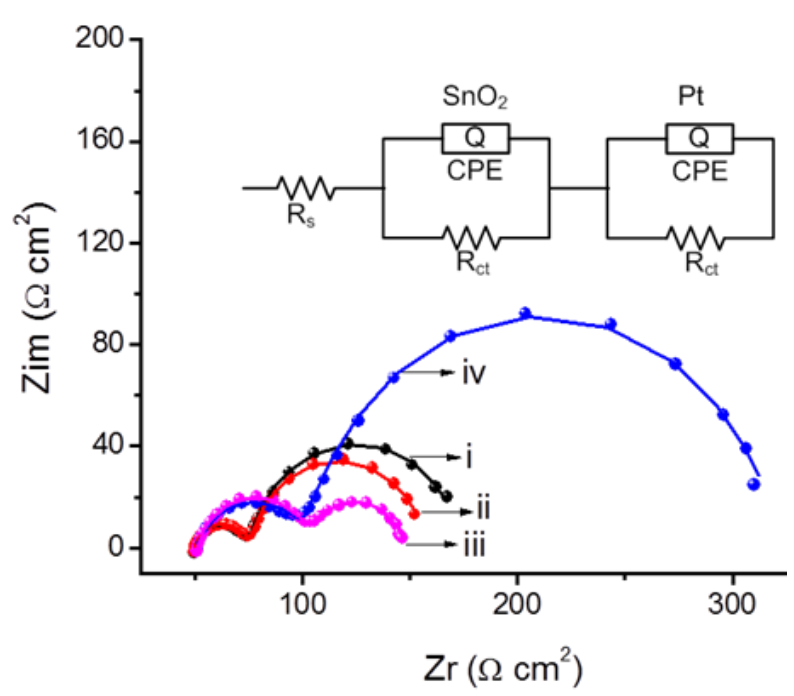

Figure 7.6: Impedance spectra (Nyquist plots) measured at the respective $V_{o c}$ of $\mathrm{ZnPcS} / \mathrm{SnO}_{2}$ and $\mathrm{ZnTMPyP}: \mathrm{ZnPcS} / \mathrm{SnO}_{2}$ in dark (i and ii) and under AM1.5 light conditions (iii and iv), respectively. The Figure inset shows equivalent circuit diagram used to fit the data.

respectively. The recombination resistance doubled for the $\mathrm{ZnPcS} / \mathrm{ZnTMPyP} / \mathrm{SnO}_{2}$ electrode to $220 \Omega \mathrm{cm}^{2}$ and $44.32 \Omega \mathrm{cm}^{2}$ under dark and light respectively. Recombination resistance of electrode determines the feasiblity of electrons being recombined with electrolyte after injection. In other words, resistance to the recombination electrons with the $\mathrm{I}_{3}{ }^{-}$ions near the electrode. Under dark condition, the concentration of $\mathrm{I}_{3}^{-}$is very low compared to light as there is no excitation of heterodimer ${ }^{314}$. This explains the reason for better performance of heterodimer compared to $\mathrm{ZnPcS}$ electrode. Better electron injection by the dimer into $\mathrm{SnO}_{2}$ upon irradiation resulted increased local concentration of $\mathrm{I}_{3}{ }^{-}$ions which inturn decreased the recombination resistance under light condition.

\subsection{Summary}

Ion paired heterodimers based on water soluble porphyrin and pthalocyanine were used as the photo electron injection source resulting in photocurrent. UV and 
steady state emission studies showed efficient electron transfer between the dimers and the strength of binding between the dimers depends on the geometry. Photoelectrochemical cells using the heterodimer decorated $\mathrm{SnO}_{2}$ showed IPCE higher than $50 \%$ over the wavelength region of $400 \mathrm{~nm}$ to $700 \mathrm{~nm}$. Overall energy efficiency of this panchromatic system was about $0.5 \%$ due to lower fill factor and open circuit potential. Moreover, the dimer showed better performance due to the higher recombination resistance as revealed by impedance spectroscopy. 


\section{CHAPTER 8}

\section{SUMMARY}

Artificial photosynthesis - it is the process of creating a charge separated positive and negative charge species between an organic donor and an acceptor molecule with the help of light. One of the main purposes of designing a charge separated donor-acceptor system is to construct photoelectrochemical devices that could be used either to generate photocurrent from sunlight to store it in an energy storage device or to generate clean fuels such as hydrogen using water and sunlight. Thus, mimicking photosynthesis to build efficient solar energy harvesting systems has become one of the most highlighted research areas.

As discussed in chapter 1, donor and acceptor molecules depending on the type of linkage between them can be classified as covalent or non-covalent interacting systems with their own merits and demerits. In order to modify surfaces to be used in photoelectrochemical devices, different techniques such as Langmuir-Blodgett (LB) films, self-assembled Monolayer on Au and ITO (SAM), and electrophoretic deposition have been reported ${ }^{11-16}$. Aided by these film forming techniques, by using intermolecular interactions of non-covalent systems, PECs have been designed to demonstrate the self-assembly of the donor and acceptor molecules. ${ }^{12,46-48}$ Although, as a proof of concept these photoelectrochemical supramolecular devices are shown to function, serious attempts have not been made to improve their efficiency. Using suitable methods and synthesized molecules, new approaches directed towards the objective of "creating the best supramolecular photovoltaic device" are being demonstrated in this thesis. 
Axial coordination between the donor and acceptor molecules was established and a cathodic photoelectochemical behavior of donor-acceptor film was observed in chapter 3. Electropolymerization of triphenyl amine substituted porphyrin $\left[\left(\mathrm{Ph}_{2} \mathrm{~N}\right)_{4} \mathrm{ZnP}\right]$ on a piezoelectric quartz crystal microbalance and on a ITO surface resulted in deposition of a conducting film. Using electrochemical quartz nanobalance setup, the mass of the $\left(\mathrm{Ph}_{2} \mathrm{~N}\right)_{4} \mathrm{ZnP}$ film deposited was found to be $863 \mathrm{ng}$. Upon axially coordinating imidazole functionalized fullerene $\left(\mathrm{C}_{60} \mathrm{im}\right)$ to the porphyrin film, the amount of coordinated fullerene was estimated to be $122 \mathrm{ng}$. This corresponds to the formation of a mono or bilayer of the donor-acceptor molecules on the surface. Optical studies of the $\left(\mathrm{Ph}_{2} \mathrm{~N}\right)_{4} \mathrm{ZnP}$ film revealed that the porphyrin $\pi$ system remained intact after electropolymerization and also the film showed characteristic changes in absorbance upon establishing axial coordination of the acceptor molecule. Photoelectrochemical cells constructed using the electropolymerized $\left(\mathrm{Ph}_{2} \mathrm{~N}\right)_{4} \mathrm{ZnP}$ film modified ITO electrode generated a cathodic photocurrent revealing the direction of electron flow towards the electrolyte. Using optimized iodide-iodine redox electrolyte, the recorded cathodic photocurrent density of the $\left(\mathrm{Ph}_{2} \mathrm{~N}\right)_{4} \mathrm{ZnP}$ film $\left(20 \mu \mathrm{A} / \mathrm{cm}^{2}\right)$ increased nearly 3 times upon axially coordinating with $\mathrm{C}_{60} \mathrm{im}$ to the $\left(\mathrm{Ph}_{2} \mathrm{~N}\right)_{4} \mathrm{ZnP}$ film. The IPCE of dyad system without any external bias voltage was found to be $2 \%$ illustrating the importance of donoracceptor in the newly constructed photocell.

A detailed study of donor-acceptor system $\left(\left(\mathrm{Ph}_{2} \mathrm{~N}\right)_{4} \mathrm{ZnP}-\mathrm{C}_{60} \mathrm{im}\right)$ based on electropolymerization led to the design and construction of photoelectrochemical systems described in chapter 4 and 5 . It also showed in real-time, the feasibility of selfassembling donor-acceptor molecules on a conducting solid surface using axial 
coordination. Later reports published on improving the performance of donor-acceptor molecules for organic solar cells using axially coordination supported these results.

Axial coordination is an act of binding axially requires a coordinating ligand and coordinatively unsaturated metal containing macrocycle such as zinc porphyrin. As showed in the chapter 4.1, we reported a novel method for immobilizing zinc porphyrin with no carboxylic acid functionality as a sensitizer for dye sensitized solar cells. With our method, fairly large molecules such as covalently linked porphyrin-ferrocene dyad were made to become possible dyes for solar cell applications. Using the same zinc porphyrin dye solution, it was visibly clear that an axially coordinated electrode appeared green compared to the red color of the dye adsorbed electrode. Of all the zinc porphyrins used for surface immobilization, zinc porphyrin-ferrocene dyad showed highest performance. Also, of all the investigated coordinating ligands, imidazole was the best due to its favorable binding properties. The better performance for zinc porphyrin-ferrocene dyad was due to the faster regeneration of zinc porphyrin (donor-1) by the ferrocene (donor-2) due to facile hole migration. The IPCE value for the axially coordinated dyad DSSCs was $36 \%$ at the Soret band $(430 \mathrm{~nm})$ and the energy conversion efficiency was $0.56 \%$ with a fill factor of $46 \%$.

Reasons for the low fill factor in the axially coordinated solar cells were unraveled in chapter 4.2. In this chapter, effect of metal ion in the porphyrin macrocycle cavity was reported using ZnTPP and MgTPP. Magnesium porphyrin is easier to oxidize compared to zinc porphyrin by about $200 \mathrm{mV}$. So it has a higher excited state oxidation potential and lower driving force for charge recombination. Also the calculated light harvesting efficiency of the MgTPP (60\% at the Soret) was higher compared to ZnTPP ( $30 \%$ at the 
Soret) upon axially coordinating on titanium dioxide surface. These factors resulted in a higher photocurrent density $\left(1.6 \mathrm{~mA} / \mathrm{cm}^{2}\right)$ and photovoltage $(0.5 \mathrm{~V})$ for MgTPP compared to ZnTPP $\left(0.19 \mathrm{~mA} / \mathrm{cm}^{2}\right.$ and $\left.0.4 \mathrm{~V}\right)$. Yet the fill factor for MgTPP immobilized electrode was around $46 \%$ similar to zinc porphyrin-ferrocene dyad reported in the previous chapter. In order to improve the fill factor and the performance, the solvent used for preparing the electrolyte was optimized in this chapter. Typically, orthodichlorobenzene is used as a solvent for studying axially coordinating intermolecular systems in solution. But using the same as an electrolyte-solvent hindered the performance of DSSCs. The lower dielectric constant of DCB resulted in higher charge transfer resistance of the counter electrode-electrolyte interface. The regeneration of the iodide-iodine mediator at the Pt counter electrode interface slowed down and resulted in lower fill factor. However, upon adding acetonitrile (polar solvent) in a very small amount, the charge transfer resistance at the Pt/electrolyte interface decreased by half (from $2610 \Omega \mathrm{cm}^{2}$ to $1558 \Omega \mathrm{cm}^{2}$ ) as shown in the electrochemical Impedance study. Here, the fill factor of MgTPP electrode system improved from $46 \%$ to $72 \%$ with an energy efficiency and IPCE of $0.6 \%$ and $26 \%(430 \mathrm{~nm})$ respectively.

Supramolecular chemistry is the chemistry of inter- and intra-molecular interactions between the preorganized molecules. Porphyrins are one of the versatile supramolecules that could be easily modified for specific applications. As shown in chapter 5, modified porphyrins could be used either as an anion binding species or as an axial coordinating site depending on the type of core modifications. We have exploited porphyrins to design DSSCs based on more than one type of intermolecular interactions. 
In chapter 5.1, bis-fullerene-substituted oxoporphyrinogen showed a $500 \mathrm{mV}$ cathodic shift in oxidation potential upon fluoride anion binding to the OXP center. As a result, increase in the life time of charge separated states in the range of 72 ps with $\mathrm{F}^{-}$ anion compared to $15 \mathrm{ps}$ without fluoride was observed. The study also led to the design of a unique dual mode binding system discussed in chapter 5.2, composed of bisporphryin-substituted oxoporphyrinogen and bipyridine appended fullerene. The supramolecular compound used in this study had an anion binding site and an axial coordinating site. UV absorption and steady state emission experiments showed a clear distinction between the two types of interactions. The axial coordination between the zinc porphyrin and $\mathrm{C}_{60}$ bipyridine had a binding constant of $1.9 \times 10^{5} \mathrm{M}^{-1}$ compared to the $K$ value of $7.4 \times 10^{4} \mathrm{M}^{-1}$ for fluoride ion binding to oxoporphyrinogen. NMR titrations confirmed that the axial binding remained intact even after addition of excess fluoride ions. After the formation of donor-acceptor, addition of anions followed a trend in the strength of binding to oxoporphyrinogen. (Fluoride $>$ Acetate $>$ Phosphate $>$ Perchlorate)

Electrochemical titrations showed that upon axial coordination there was about $20 \mathrm{mV}$ shift in oxidation potential of zinc porphyrin while the oxidation potential of oxoporphyrinogen remain unaffected. At the same time, the oxidation potential of the zinc porphyrin remained unaffected while oxoporphyrinogen became easy to oxidize (from $0.48 \mathrm{~V}$ to $-0.12 \mathrm{~V}$ ) upon adding fluoride anion. Transient absorption studies also showed the influence of anion binding on the life time of the charge separated states. For example, the triplet charge separated states between the donor and the acceptor had a lifetime of $14 \mu \mathrm{s}$ in the presence of fluoride ion compared to $0.16 \mu \mathrm{s}$ in absence of $\mathrm{F}^{-}$anion. 
In chapter 5.3, we have shown that an anion binding phenomenon could also be used as a tool for improving the performance of solar cells. Supported by our solution studies, a solar cell that was anion bound showed improved performance compared to the control cells. The photocurrent density doubled and IPCE got improved from 2 to $4 \%$ due to anion binding.

Axial ligation has opened new ways to sensitize semiconducting metal oxide surfaces using unconventional dye molecules. Yet, this approach has its limitations on the design of dye molecules that could adsorb entire visible spectrum. Moreover, the efficiency of the system depends on the size of the coordinating dye molecule and its proclivity toward axial binding. To build a panchromatic light harvesting supramolecular solar cells, we investigated other useful supramolecular interactions. Of all, ion-pairing between the oppositely charged ions seem promising and attractive. One such system and its photoelectrochemical properties were described in chapter 6 .

Modification of the ring periphery of the porphyrins with positively charged functional groups made it water soluble and they have been used for several applications. There are reports on water soluble porphyrin interactions with the metal clusters but in our study its application for generating photocurrent is demonstrated. We established that there is a strong interaction between the positively charged porphyrins and negatively charged tin oxide using UV absorption studies. The steady state emission and lifetime measurements revealed that the strong interactions between them resulted in a photoinduced electron transfer from porphyrin to the metal oxide. Use of smaller cation such as $\mathrm{Li}^{+}$resulted in dye desorption from the surface and validated the ion-ion interaction between the metal oxides and the dyes. Photoelectrochemical cells 
based on water soluble porphyrins resulted in the highest IPCE reported for the supramolecular systems. Of all the positively charged porphyrins, ZnTMPyP showed an IPCE of $90 \%$ near $450 \mathrm{~nm}$ and was better than its corresponding free base porphyrin due to better charge recombination resistance and higher lighter harvesting efficiency. Another important factor attributed to ZnTMPyP modified electrode's better performance compared to other water soluble porphyrins was due to the flattening of the pyridyl ring upon adsorbing on tin oxide. Hence in chapter 6, reported a near unity IPCE photoelectrochemical cell based purely on electrostatic attractions and demonstrated the importance of supramolecular chemistry to demonstrate new ways of dye sensitization.

In Chapter 7, we showed an ion-paired heterodimer sensitized photoelectrochemical cell efficiently converting the $400-700 \mathrm{~nm}$ visible light into photocurrent. Heterodimers were composed of positively charged porphyrin and negatively charged phthalocyanine molecules. Spectroscopic titrations showed stronger binding as well as emission quenching upon dimer formation. Photocurrent density of heterodimer PECs was higher than the individual monomer dye cells signifying the importance of dimer formation. Electrochemical impedance study showed that upon dimer formation there was an increase in the recombination resistance adding up to the improved performance. IPCE of $50 \%$ over the entire range from $400-700 \mathrm{~nm}$ was obtained for this photoelectrochemical system designed using supramolecular technique. Still, the energy efficiency of the system was low $(0.5 \%)$ due to lower open circuit potential and fill factor. 
Overall, we have demonstrated here in this thesis, the design and construction of supramolecular solar cells using axial ligation, anion binding and ion-ion pairing approaches. The results that were obtained in this study were encouraging and motivating to create next generation of photovoltaic devices using for the supramolecular concepts. The notion to create and to design photoelectrochemical cells to house one or more supramolecules for solar cells would generate new ideas leading to unconventional design. So the vision of building affordable efficient solid state photovoltaic devices seems promising. 
APPENDIX

LIST OF PUBLICATIONS 
I. Chandra K. C.; Subbaiyan, N. K.; D'Souza, F.,"Supramolecular Donor-Acceptor Assembly Derived from Tetracarbazole-Zinc Phthalocyanine Coordinated to Fullerene: Design, Synthesis, Photochemical and Photoelectrochemcial Studies." J. Phys. Chem. C, 2012, 116(22), pp 11964-11972.

II. Wang, F.; Subbaiyan, N. K.; Wang, Q.; Rochford, C.; Xu, G.; Lu, R.; Elliot, A.; D’Souza, F.; Hui, R.; Wu, J., "Development of Nanopatterned Fluorine-Doped Tin Oxide Electrodes for Dye-Sensitized Solar Cells with Improved Light Trapping." ACS Appl. Mater. Inter. 2012, 4 (3), 1565-1572.

III. Hill, J. P.; Subbaiyan, N. K.; D'Souza, F.; Xie, Y.; Sahu, S.; Sanchez-Ballester, N. M.; Richards, $\quad$ G. J.; Mori, $\quad$ T.; Ariga, K., "Antioxidant-substituted tetrapyrazinoporphyrazine as a fluorescent sensor for basic anions." Chem. Commun. 2012, 48 (33), 3951-3953.

IV. Subbaiyan, N. K.; D'Souza, F., "Light-to-electron converting panchromatic supramolecular solar cells of phthalocyanine-porphyrin heterodimers adsorbed onto nanocrystalline SnO2 electrodes." Chem. Commun. 2012, 48 (30), 3641-3643.

V. Subbaiyan, N. K.; Maligaspe, E.; D'Souza, F.: "Near Unity Photon-to-Electron Conversion Efficiency of Photoelectrochemical Cells Built on Cationic Water-Soluble Porphyrins Electrostatically Decorated onto Thin-Film Nanocrystalline $\mathrm{SnO}(2)$ Surface." ACS Appl. Mater. Inter. 2011, 3, 2368-2376.

VI. Subbaiyan, N. K.; Hill, J. P.; Ariga, K.; Fukuzumi, S.; D'Souza, F.: "Enhanced photocurrents via redox modulation by fluoride binding to oxoporphyrinogen in a zinc porphyrin-oxoporphyrinogen surface modified $\mathrm{TiO}(2)$ supramolecular solar cell". Chem. Commun. 2011, 47, 6003-6005. 
VII. Schley, N. D.; Blakemore, J. D.; Subbaiyan, N. K.; Incarvito, C. D.; D'Souza, F.; Crabtree, R. H.; Brudvig, G. W.: "Distinguishing Homogeneous from Heterogeneous Catalysis in Electrode-DrivenWater Oxidation with Molecular Iridium Complexes". J. Am. Chem. Soc. 2011, 133, 10473-10481

VIII. Sandanayaka, A. S. D.; Subbaiyan, N. K.; Das, S. K.; Chitta, R.; Maligaspe, E.; Hasobe, T.; Ito, O.; D'Souza, F.: "Diameter-Sorted SWCNT-Porphyrin and SWCNTPhthalocyanine Conjugates for Light-Energy Harvesting". Chem. Phys. Chem. 2011, 12, 2266-2273.

IX. $\quad$ Das, S. K.; Subbaiyan, N. K.; D'Souza, F.; Sandanayaka, A. S. D.; Hasobe, T.; Ito, O.: "Photoinduced processes of the supramolecularly functionalized semiconductive SWCNTs with porphyrins via ion-pairing interactions."Energ. Environ. Sci. $2011,4,707-716$.

X. Amin, A. N.; El-Khouly, M. E.; Subbaiyan, N. K.; Zandler, M. E.; Supur, M.; Fukuzumi, S.; D'Souza, F.: "Syntheses, Electrochemistry, and Photodynamics of Ferrocene-Azadipyrromethane Donor-Acceptor Dyads and Triads." J. Phys. Chem. A $2011,115,9810-9819$.

XI. D'Souza, F.; Amin, A. N.; El-Khouly, M. E.; Subbaiyan, N. K.; Zandler, M. E.; Fukuzumi, S., "Control over Photoinduced Energy and Electron Transfer in Supramolecular Polyads of Covalently linked azaBODIPY-Bisporphyrin 'Molecular Clip' Hosting Fullerene." J. Am. Chem. Soc. 2011, 134 (1), 654-664

XII. Wijesinghe, C. A.; Niemi, M.; Tkachenko, N. V.; Subbaiyan, N. K.; Zandler, M. E.; Lemmetyinen, H.; D'Souza, F.: "Photoinduced electron transfer in a directly linked 
meso-triphenylamine zinc porphyrin-quinone dyad." J. Porphyr. Phthalocya. 2011, 15, $391-400$.

XIII. Wijesinghe, C. A.; El-Khouly, M. E.; Subbaiyan, N. K.; Supur, M.; Zandler, M. E.; Ohkubo, K.; Fukuzumi, S.; D'Souza, F.: "Photochemical Charge Separation in Closely Positioned Donor-Boron Dipyrrin-Fullerene Triads." Chem-Eur. J. 2011, 17, 3147-3156.

XIV. Maligaspe, E.; Kumpulainen, T.; Lemmetyinen, H.; Tkachenko, N. V.; Subbaiyan, N. K.; Zandler, M. E.; D'Souza, F.: "Ultrafast Singlet-Singlet Energy Transfer in SelfAssembled via Metal-Ligand Axial Coordination of Free-Base Porphyrin-Zinc Phthalocyanine and Free-Base Porphyrin-Zinc Naphthalocyanine Dyads." J. Phys. Chem. A 2010, 114, 268-277.

XV. $\quad$ D'Souza, F.; Maligaspe, E.; Sandanayaka, A. S. D.; Subbaiyan, N. K.; Karr, P. A.; Hasobe, T.; Ito, O.: "Photochemical Charge Separation in Supramolecular Phthalocyanine-Multifullerene Conjugates Assembled by Crown Ether-Alkyl Ammonium Cation Interactions." J. Phys. Chem. A 2010, 114, 10951-10959.

XVI. Subbaiyan, N. K.; Hill, J. P.; El-Khouly, M. E.; Charvet, R.; Ariga, K.; Fukuzumi, S.; D'Souza, F.: "Effect of anion binding on charge stabilization in a bis-fullereneoxoporphyrinogen conjugate." Chem. Commun. 2010, 46, 7933-7935

XVII. Subbaiyan, N. K.; Wijesinghe, C. A.; D'Souza, F.: "Supramolecular Solar Cells: Surface Modification of Nanocrytalline $\mathrm{TiO}(2)$ with Coordinating Ligands To Immobilize Sensitizers and Dyads via Metal-Ligand Coordination for Enhanced Photocurrent Generation.” J. Am. Chem. Soc. 2009, 131, 14646-+.

XVIII. Subbaiyan, N. K.; Obraztsov, L.; Wijesinghe, C. A.; Tran, K.; Kutner, W.; D'Souza, F.: "Supramolecular Donor-Acceptor Hybrid of Electropolymerized Zinc 
Porphyrin with Axially Coordinated Fullerene: Formation, Characterization, and Photoelectrochemical Properties." J. Phys. Chem. C 2009, 113, 8982-8989.

XIX. Sandanayaka, A. S. D.; Chitta, R.; Subbaiyan, N. K.; D'Souza, L.; Ito, O.; D'Souza, F.: "Photoinduced Charge Separation in Ion-Paired Porphyrin-Single-Wall Carbon Nanotube Donor-Acceptor Hybrids." J. Phys. Chem. C 2009, 113, 13425-13432. XX. Maligaspe, E.; Tkachenko, N. V.; Subbaiyan, N. K.; Chitta, R.; Zandler, M. E.; Lemmetyinen, H.; D'Souza, F.: "Photosynthetic Antenna-Reaction Center Mimicry: Sequential Energy- and Electron Transfer in a Self-assembled Supramolecular Triad Composed of Boron Dipyrrin, Zinc Porphyrin and Fullerene.” J. Phys. Chem. A 2009, $113,8478-8489$.

XXI. D'Souza, F.; Venukadasula, G. M.; Yamanaka, K.; Subbaiyan, N. K.; Zandler, M. E.; Ito, O.: "Through-bond photoinduced electron transfer in a porphyrin-fullerene conjugate held by a Hamilton type hydrogen bonding motif." Org. Biomol. Chem. 2009, 7, 1076-1080.

XXII. D'Souza, F.; Subbaiyan, N. K.; Xie, Y. S.; Hill, J. P.; Ariga, K.; Ohkubo, K.; Fukuzumi, S.: "Anion-Complexation-Induced Stabilization of Charge Separation." J. Am. Chem. Soc. 2009, 131, 16138-16146.

XXIII. D'Souza, F.; Maligaspe, E.; Ohkubo, K.; Zandler, M. E.; Subbaiyan, N. K.; Fukuzumi, S.: "ORGN 325-Photosynthetic reaction center mimicry: Charge stabilization in self-assembled cofacial zinc phthalocyanine dimer - fullerene conjugates." Abstr Pap Am Chem S 2009, 237.

XXIV. D'Souza, F.; Maligaspe, E.; Ohkubo, K.; Zandler, M. E.; Subbaiyan, N. K.; Fukuzumi, S.: "Photosynthetic Reaction Center Mimicry: Low Reorganization Energy 
Driven Charge Stabilization in Self-Assembled Cofacial Zinc Phthalocyanine DimerFullerene Conjugate." J. Am. Chem. Soc. 2009, 131, 8787-8797.

XXV. Richards, G. J.; Hill, J. P.; Subbaiyan, N. K.; D'Souza, F.; Karr, P. A.; Elsegood, M. R. J.; Teat, S. J.; Mori, T.; Ariga, K.: "Pyrazinacenes: Aza Analogues of Acenes." J. Org. Chem. 2009, 74, 8914-8923.

XXVI. Sandanayaka, A. S. D.; Subbaiyan, N. K.; Chitta, R.; Araki, Y.; Ito, O.; D'Souza, F.: "Co-facial magnesium porphyrin dimer complexed with fullerene: photosynthetic reaction center model of 'special pair' self-assembled to electron acceptor." J. Porphyr. Phthalocya. 2008, 12, 857-865.

XXVII. D'Souza, F.; Maligaspe, E.; Zandler, M. E.; Subbaiyan, N. K.; Ohkubo, K.; Fukuzumi, S.: "Metal Quinolinolate-Fullerene(s) Donor-Acceptor Complexes: Evidence for Organic LED Molecules Acting as Electron Donors in Photoinduced ElectronTransfer Reactions.” J. Am. Chem. Soc. 2008, 130, 16959-16967.

XXVIII. D'Souza, F.; Chitta, R.; Ohkubo, K.; Tasior, M.; Subbaiyan, N. K.; Zandler, M. E.; Rogacki, M. K.; Gryko, D. T.; Fukuzumi, S.: "Corrole-Fullerene Dyads: Formation of Long-Lived Charge-Separated States in Nonpolar Solvents." J. Am. Chem. Soc. 2008, 130, 14263-14272.

XXIX. Gadde, S.; Islam, D. M. S.; Wijesinghe, C. A.; Subbaiyan, N. K.; Zandler, M. E.; Araki, Y.; Ito, O.; D'Souza, F.: "Light-induced electron transfer of a supramolecular bis(zinc porphyrin)-fullerene triad constructed via a diacetylamidopyridine/uracil hydrogen-bonding motif." J. Phys. Chem. C 2007, 111, 12500-12503.

XXX. D'Souza, F.; Chitta, R.; Sandanayaka, A. S. D.; Subbaiyan, N. K.; D'Souza, L.; Araki, Y.; Ito, O.: "Self-assembled single-walled carbon nanotube : zinc-porphyrin 
hybrids through ammonium ion-crown ether interaction: Construction and electron transfer." Chem-Eur. J. 2007, 13, 8277-8284.

XXXI. D'Souza, F.; Chitta, R.; Sandanayaka, A. S. D.; Subbaiyan, N. K.; D'Souza, L.; Araki, Y.; Ito, O.: "Supramolecular carbon nanotube-fullerene donor-acceptor hybrids for photoinduced electron transfer." J. Am. Chem. Soc. 2007, 129, 15865-15871. 


\section{LIST OF REFERENCES}

(1) Steed, J. W.; Atwood, J. L. Supramolecular chemistry; Wiley: Chichester 2005.

(2) Balzani, V. Electron transfer in chemistry; Wiley-VCH: Weinheim; New York, 2001.

(3) Deisenhofer, J.; Norris, J. R. The Photosynthetic reaction center; Academic Press: San Diego, CA, 1993.

(4) Kalyanasundaram, K. Dye-sensitized solar cells; CRC Press: Boca Raton, Fla., 2010.

(5) Kavarnos, G. J. Fundamentals of photoinduced electron transfer; VCH Publishers: New York, NY, 1993.

(6) Mataga, N., Miyasaka, H. Electron transfer Wiley: New York; Chichester; Weinheim, 1999.

(7) Rehm, D.; Weller, A. Kinetics and mechanism of electron transfer in florescence quenching in acetonitrile, 1969.

(8) Bard, A. J.; Fox, M. A. Acc. Chem. Res. 1995, 28, 141.

(9) Bard, A. J.; Faulkner, L. R. Electrochemical methods : fundamentals and applications; Wiley: New York, 2001.

(10) Gratzel, M. Nature 2001, 414, 338.

(11) Gust, D.; Moore, T. A.; Intermolecular photoinduced electron-transfer reactions of pophyrins; Academic Press: 2000; Vol. 8, p 153.

(12) Wasielewski, M. R. Chem. Rev. 1992, 92, 435.

(13) D'Souza, F.; Ito, O. Electron transfer in self-assembled supramolecular fullerene based donor-acceptor conjugates; American Scientific Publishers; 2008; Vol. 1, p 485.

(14) Fukuzumi, S. Phys. Chem. Chem. Phys. 2008, 10, 2283.

(15) Kamat, P. V., Guldi, D Fullerenes : chemistry, physics, and technology; WileyInterscience: New York, 2000.

(16) Imahori, H. Org. Biomol. Chem. 2004, 2, 1425. 
(17) Imahori, H.; Guldi, D. M.; Tamaki, K.; Yoshida, Y.; Luo, C.; Sakata, Y.; Fukuzumi, S. J. Am. Chem. Soc. 2001, 123, 6617.

(18) D'Souza, F.; Smith, P. M.; Gadde, S.; McCarty, A. L.; Kullman, M. J.; Zandler, M. E.; Ito. U, M.; Araki, Y.; Ito, O. J. Phys. Chem. B 2004, 108, 11333.

(19) Zhou, D.; Gan, L.; Luo, C.; Tan, H.; Huang, C.; Yao, G.; Zhao, X.; Liu, Z.; Xia, X.; Zhang, P. J. Phys. Chem. 1996, 100, 3150.

(20) Vuorinen, T.; Kaunisto, K.; Tkachenko, N. V.; Efimov, A.; Lemmetyinen, H.; Alekseev, A. S.; Hosomizu, K.; Imahori, H. Langmuir 2005, 21, 5383.

(21) Conoci, S.; Guldi, D. M.; Nardis, S.; Paolesse, R.; Kordatos, K.; Prato, M.; Ricciardi, G.; Vicente, M. G. H.; Zilbermann, I.; Valli, L. Chem.Eur. J. 2004, 10, 6523.

(22) Tkachenko, N. V.; Vuorimaa, E.; Kesti, T.; Alekseev, A. S.; Tauber, A. Y.; Hynninen, P. H.; Lemmetyinen, H. J. Phys. Chem. B 2000, 104, 6371.

(23) Taniguchi, T.; Fukasawa, Y.; Miyashita, T. J. Phys. Chem. B 1999, 103, 1920.

(24) Jin, J.; Li, L. S.; Li, Y.; Zhang, Y. J.; Chen, X.; Wang, D.; Jiang, S.; Li, T. J.; Gan, L. B.; Huang, C. H. Langmuir 1999, 15, 4565.

(25) Tran-Thi, T.-H.; Palacin, S.; Clergeot, B. Chem. Phys. Lett. 1989, 157, 92.

(26) Imahori, H.; Azuma, T.; Ozawa, S.; Yamada, H.; Ajavakom, A.; Norieda, H.; Sakata, Y.; Ushida, K. Chem. Commun. (Cambridge) 1999, 557.

(27) Imahori, H.; Azuma, T.; Ajavakom, A.; Norieda, H.; Yamada, H.; Sakata, Y. J. Phys. Chem. B 1999, 103, 7233.

(28) Enger, O.; Nuesch, F.; Fibbioli, M.; Echegoyen, L.; Pretsch, E.; Diederich, F. J. Mater. Chem. 2000, 10, 2231.

(29) Hirayama, D.; Takimiya, K.; Aso, Y.; Otsubo, T.; Hasobe, T.; Yamada, H.; Imahori, H.; Fukuzumi, S.; Sakata, Y. J. Am. Chem. Soc. 2002, 124, 532.

(30) Hirayama, D.; Yamashiro, T.; Takimiya, K.; Aso, Y.; Otsubo, T.; Norieda, H.; Imahori, H.; Sakata, Y. Chem. Lett. 2000, 570.

(31) Yamada, H.; Imahori, H.; Nishimura, Y.; Yamazaki, I.; Ahn, T. K.; Kim, S. K.; Kim, D.; Fukuzumi, S. J. Am. Chem. Soc. 2003, 125, 9129.

(32) Imahori, H.; Yamada, H.; Nishimura, Y.; Yamazaki, I.; Sakata, Y. J. Phys. Chem. B 2000, 104, 2099. 
(33) Imahori, H.; Yamada, H.; Ozawa, S.; Sakata, Y.; Ushida, K. Chem. Commun. (Cambridge) 1999, 1165.

(34) Akiyama, T.; Imahori, H.; Ajawakom, A.; Sakata, Y. Chem. Lett. 1996, 907.

(35) Imahori, H.; Kimura, M.; Hosomizu, K.; Sato, T.; Ahn, T. K.; Kim, S. K.; Kim, D.; Nishimura, Y.; Yamazaki, I.; Araki, Y.; Ito, O.; Fukuzumi, S. Chem.Eur. J. 2004, 10, 5111.

(36) Kim, K.-S.; Kang, M.-S.; Ma, H.; Jen, A. K. Y. Chem. Mater. 2004, 16, 5058.

(37) Barazzouk, S.; Hotchandani, S.; Kamat, P. V. Adv. Mater. (Weinheim, Ger.) 2001, 13, 1614.

(38) Kamat, P. V.; Barazzouk, S.; Thomas, K. G.; Hotchandani, S. J. Phys. Chem. B 2000, 104, 4014.

(39) Kira, A.; Umeyama, T.; Matano, Y.; Yoshida, K.; Isoda, S.; Isosomppi, M.; Tkachenko, N. V.; Lemmetyinen, H.; Imahori, H. Langmuir 2006, 22, 5497.

(40) Hasobe, T.; Imahori, H.; Fukuzumi, S.; Kamat, P. V. J. Mater. Chem. 2003, 13, 2515.

(41) Hasobe, T.; Imahori, H.; Fukuzumi, S.; Kamat, P. V. J. Phys. Chem. B 2003, 107, 12105.

(42) Biju, V.; Barazzouk, S.; Thomas, K. G.; George, M. V.; Kamat, P. V. Langmuir 2001, 17, 2930.

(43) Okamoto, K.; Hasobe, T.; Tkachenko, N. V.; Lemmetyinen, H.; Kamat, P. V.; Fukuzumi, S. J. Phys. Chem. A 2005, 109, 4662.

(44) Hasobe, T.; Hattori, S.; Kotani, H.; Ohkubo, K.; Hosomizu, K.; Imahori, H.; Kamat, P. V.; Fukuzumi, S. Org. Lett. 2004, 6, 3103.

(45) Imahori, H. J. Mater. Chem. 2007, 17, 31.

(46) Hasobe, T. Phys. Chem. Chem. Phys. 2010, 12, 44.

(47) D'Souza, F.; Kadish, K. M., Smith, K. M., Guilard, R. Handbook of porhyrin science with application to chemistry, physics, materials science, engineering, biology and medicine. Volume 1 Supramolecular chemistry; World Scientific Pub. Co.: Singapore; Hackensack, N.J., 2010.

(48) Bonifazi, D.; Enger, O.; Diederich, F. Chem. Soc. Rev. 2007, 36, 390. 
(49) Guldi, D. M.; Zilbermann, I.; Anderson, G.; Li, A.; Balbinot, D.; Jux, N.; Hatzimarinaki, M.; Hirsch, A.; Prato, M. Chem. Commun. (Cambridge, U. K.) 2004, 726.

(50) Guldi, D. M.; Prato, M. Chem. Commun. (Cambridge, U. K.) 2004, 2517.

(51) Zilbermann, I.; Anderson, G. A.; Guldi, D. M.; Yamada, H.; Imahori, H.; Fukuzumi, S. J. Porphyrins Phthalocyanines 2003, 7, 357.

(52) Zilbermann, I.; Lin, A.; Hatzimarinaki, M.; Hirsch, A.; Guldi, D. M. Chem. Commun. 2004, 96.

(53) Mwaura, J. K.; Pinto, M. R.; Witker, D.; Ananthakrishnan, N.; Schanze, K. S.; Jr, R. Langmuir 2005, 21, 10119.

(54) Imahori, H.; Tkachenko, N. V.; Vehmanen, V.; Tamaki, K.; Lemmetyinen, H.; Sakata, Y.; Fukuzumi, S. J. Phys. Chem. A 2001, 105, 1750.

(55) Tkachenko, N. V.; Guenther, C.; Imahori, H.; Tamaki, K.; Sakata, Y.; Fukuzumi, S.; Lemmetyinen, H. Chem. Phys. Lett. 2000, 326, 344.

(56) Imahori, H.; Liu, J.-C.; Hotta, H.; Kira, A.; Umeyama, T.; Matano, Y.; Li, G.; Ye, S.; Isosomppi, M.; Tkachenko, N. V.; Lemmetyinen, H. J. Phys. Chem. B 2005, 109, 18465.

(57) Huang, C.-H.; McClenaghan, N. D.; Kuhn, A.; Hofstraat, J. W.; Bassani, D. M. Org. Lett. 2005, 7, 3409.

(58) Imahori, H.; Liu, J.-C.; Hosomizu, K.; Sato, T.; Mori, Y.; Hotta, H.; Matano, Y.; Araki, Y.; Ito, O.; Maruyama, N.; Fujita, S. Chem. Commun. (Cambridge, U. K.) 2004, 2066.

(59) Liu, Y.; Xiao, S.; Li, H.; Li, Y.; Liu, H.; Lu, F.; Zhuang, J.; Zhu, D. J. Phys. Chem. B 2004, 108, 6256.

(60) D'Souza, F.; Ito, O. Coord. Chem. Rev. 2005, 249, 1410.

(61) Marczak, R.; Sgobba, V.; Kutner, W.; Gadde, S.; D'Souza, F.; Guldi, D. M. Langmuir 2007, 23, 1917.

(62) Kira, A.; Umeyama, T.; Matano, Y.; Yoshida, K.; Isoda, S.; Park, J. K.; Kim, D.; Imahori, H. J. Am. Chem. Soc. 2009, 131, 3198.

(63) Hasobe, T.; Saito, K.; Kamat, P. V.; Troiani, V.; Qiu, H. J.; Solladie, N.; Kim, K. S.; Park, J. K.; Kim, D.; D'Souza, F.; Fukuzumi, S. J. Mat. Chem. 2007, 17, 4160. 
(64) Poddutoori, P. K.; Sandanayaka, A. S. D.; Zarrabi, N.; Hasobe, T.; Ito, O.; van der Est, A. J. Phys. Chem. A 2010, 115, 709.

(65) Hasobe, T.; Kamat, P. V.; Troiani, V.; Solladie, N.; Ahn, T. K.; Kim, S. K.; Kim, D.; Kongkanand, A.; Kuwabata, S.; Fukuzumi, S. J. Phys. Chem. B 2005, 109, 19.

(66) Hasobe, T.; Imahori, H.; Kamat, P. V.; Ahn, T. K.; Kim, S. K.; Kim, D.; Fujimoto, A.; Hirakawa, T.; Fukuzumi, S. J. Am. Chem. Soc. 2005, 127, 1216.

(67) Hasobe, T.; Imahori, H.; Kamat, P. V.; Fukuzumi, S. J. Am. Chem. Soc. 2003, $125,14962$.

(68) Imahori, H.; Fujimoto, A.; Kang, S.; Hotta, H.; Yoshida, K.; Umeyama, T.; Matano, Y.; Isoda, S.; Isosomppi, M.; Tkachenko, N. V.; Lemmetyinen, H. Chem.Eur. J. 2005, 11, 7265.

(69) Imahori, H.; Fujimoto, A.; Kang, S.; Hotta, H.; Yoshida, K.; Umeyama, T.; Matano, Y.; Isoda, S. Adv. Mater. (Weinheim, Ger.) 2005, 17, 1727.

(70) Hasobe, T.; Kamat, P. V.; Absalom, M. A.; Kashiwagi, Y.; Sly, J.; Crossley, M. J.; Hosomizu, K.; Imahori, H.; Fukuzumi, S. J. Phys. Chem. B 2004, 108, 12865.

(71) Guldi, D. M.; Zilbermann, I.; Anderson, G. A.; Kordatos, K.; Prato, M.; Tafuro, R.; Valli, L. J. Mater. Chem. 2004, 14, 303.

(72) Guldi, D. M.; Pellarini, F.; Prato, M.; Granito, C.; Troisi, L. Nano Lett. 2002, 2, 965.

(73) Ikeda, A.; Hatano, T.; Shinkai, S.; Akiyama, T.; Yamada, S. J. Am. Chem. Soc. 2001, 123, 4855.

(74) Mishra, A.; Fischer, M. K. R.; Bauerle, P. Angew. Chem., Int. Ed. 2009, 48, 2474.

(75) Kalyanasundaram, K.; Gratzel, M. Coord. Chem. Rev. 1998, 177, 347.

(76) Nazeeruddin, M. K.; Kay, A.; Rodicio, I.; Humphry-Baker, R.; Mueller, E.; Liska, P.; Vlachopoulos, N.; Graetzel, M. J. Am. Chem. Soc. 1993, 115, 6382.

(77) Ardo, S.; Meyer, G. J. Chem. Soc. Rev. 2009, 38, 115.

(78) Zhang, G.; Bala, H.; Cheng, Y.; Shi, D.; Lv, X.; Yu, Q.; Wang, P. Chem. Commun. 2009, 2198.

(79) Bessho, T.; Zakeeruddin, S. M.; Yeh, C. Y.; Diau, E. W. G.; Gratzel, M. Angew. Chem. Int. Edit. 2010, 49, 6646. 
(80) Yum, J. H.; Hardin, B. E.; Hoke, E. T.; Baranoff, E.; Zakeeruddin, S. M.; Nazeeruddin, M. K.; Torres, T.; McGehee, M. D.; Gratzel, M. Chem. Phys. Chem. 2011, $12,657$.

(81) Warnan, J.; Buchet, F.; Pellegrin, Y.; Blart, E.; Odobel, F. Org Lett 2011, 13, 3944.

(82) Qi, D. D.; Jiang, J. Z. J. Phys. Chem. A 2011, 115, 13811.

(83) Maeda, T.; Hamamura, Y.; Miyanaga, K.; Shima, N.; Yagi, S.; Nakazumi, H. Org. Lett. 2011, 13, 5994.

(84) Braga, A.; Giménez, S.; Concina, I.; Vomiero, A.; Mora-Seró, I. n. J. Phys. Chem. Lett. 2011, 2, 454.

(85) Kuang, D.; Walter, P.; Nüesch, F.; Kim, S.; Ko, J.; Comte, P.; Zakeeruddin, S. M.; Nazeeruddin, M. K.; Grätzel, M. Langmuir 2007, 23, 10906.

(86) Cid, J. J.; Yum, J. H.; Jang, S. R.; Nazeeruddin, M. K.; Ferrero, E. M.; Palomares, E.; Ko, J.; Gratzel, M.; Torres, T. Angew. Chem. Int. Edit. 2007, 46, 8358.

(87) Ito, S.; Chen, P.; Comte, P.; Nazeeruddin, M. K.; Liska, P.; Pechy, P.; Gratzel, M. Prog. Photovoltaics 2007, 15, 603.

(88) Bedja, I.; Hotchandani, S.; Kamat, P. V. J. Phys. Chem. 1994, 98, 4133.

(89) Hishikawa, Y.; Yanagida, M.; Koide, N. In Photovoltaic Specialists Conference, 2005. Conference Record of the Thirty-first IEEE 2005, p 67.

(90) Benesi, H. A.; Hildebrand, J. H. J. Am. Chem. Soc. 1949, 71, 2703.

(91) De, I. C. J. L. D.; Hahn, U.; Nierengarten, J.-F. Tetra. Lett. 2006, 47, 3715.

(92) Sanchez, L.; Martin, N.; Guldi, D. M. Angew. Chem., Int. Ed. 2005, 44, 5374.

(93) El-Khouly, M. E.; Ito, O.; Smith, P. M.; D'Souza, F. J. Photochem. Photobiol., C 2004, 5, 79.

(94) Guldi, D. M. Chem. Soc. Rev. 2002, 31, 22.

(95) Gust, D.; Moore, T. A.; Moore, A. L. Covalently linked systems contatinaing porphyrin units; Wiley-VCH Verlag GmbH: 2001; Vol. 3, p 272.

(96) Fukuzumi, S.; Guldi, D. M. Electron - transfer chemistry of fullerenes; Wiley-VCH Verlag GmbH: 2001; Vol. 2, p 270. 
(97) Eckert, J.-F.; Nicoud, J.-F.; Nierengarten, J.-F.; Liu, S.-G.; Echegoyen, L.; Barigelletti, F.; Armaroli, N.; Ouali, L.; Krasnikov, V.; Hadziioannou, G. J. Am. Chem. Soc. 2000, 122, 7467.

(98) Martin, N.; Sanchez, L.; Illescas, B.; Perez, I. Chem. Rev. (Washington, D. C.) 1998, 98, 2527.

(99) Imahori, H.; Sakata, Y. Adv. Mater. (Weinheim, Ger.) 1997, 9, 537.

(100) Buttry, D. A.; Ward, M. D. Chem. Rev. 1992, 92, 1355.

(101) Yeh, S. J.; Tsai, C. Y.; Huang, C.-Y.; Liou, G.-S.; Cheng, S.-H. Electrochem. Commun. 2003, 5, 373.

(102) Seo, E. T.; Nelson, R. F.; Fritsch, J. M.; Marcoux, L. S.; Leedy, D. W.; Adams, R. N. J. Am. Chem. Soc. 1966, 88, 3498.

(103) Kim, M.-J.; Seo, E.-M.; Vak, D.; Kim, D.-Y. Chem. Mater. 2003, 15, 4021.

(104) Gao, G. Y.; Chen, Y.; Zhang, X. P. J. Org. Chem. 2003, 68, 6215.

(105) Son, J. M.; Mori, T.; Ogino, K.; Sato, H.; Ito, Y. Macromolecules 1999, 32, 4849.

(106) Tamoto, N.; Adachi, C.; Nagai, K. Chem. Mater. 1997, 9, 1077.

(107) Chen, C.-T.; Lin, J.-S.; Moturu, M. V. R. K.; Lin, Y.-W.; Yi, W.; Tao, Y.-T.; Chien, C.-H. Chem. Commun. (Cambridge, U. K.) 2005, 3980.

(108) Sengupta, S.; Sadhukhan, S. K.; Muhuri, S. Tetra. Lett. 2002, 43, 3521.

(109) Wang, Y. Z.; Epstein, A. J. Acc. Chem. Res. 1999, 32, 217.

(110) Yen, W.-N.; Lo, S.-S.; Kuo, M.-C.; Mai, C.-L.; Lee, G.-H.; Peng, S.-M.; Yeh, C.-Y. Org. Lett. 2006, 8, 4239.

(111) Li, B.; Xu, X.; Sun, M.; Fu, Y.; Yu, G.; Liu, Y.; Bo, Z. Macromolecules 2006, 39, 456.

(112) Huang, C.-W.; Chiu, K. Y.; Cheng, S.-H. Dalton Trans. 2005, 2417.

(113) Clifford, J. N.; Yahioglu, G.; Milgrom, L. R.; Durrant, J. R. Chem. Commun. (Cambridge, U. K.) 2002, 1260.

(114) Frampton, M. J.; Beavington, R.; Lupton, J. M.; Samuel, I. D. W.; Burn, P. L. Synth. Met. 2001, 121, 1671. 
(115) D'Souza, F.; Gadde, S.; Islam, D. M. S.; Wijesinghe, C. A.; Schumacher, A. L.; Zandler, M. E.; Araki, Y.; Ito, O. J. Phys. Chem. A 2007, 111, 8552.

(116) Thomas, S. W., III; Joly, G. D.; Swager, T. M. Chem. Rev. (Washington, DC, U. S.) 2007, 107, 1339.

(117) McQuade, D. T.; Pullen, A. E.; Swager, T. M. Chem. Rev. (Washington, D. C.) 2000, 100, 2537.

(118) Roncali, J. Chem. Soc. Rev. 2005, 34, 483.

(119) Coakley, K. M.; McGehee, M. D. Chem. Mater. 2004, 16, 4533.

(120) Cravino, A.; Sariciftci, N. S. J. Mater. Chem. 2002, 12, 1931.

(121) Brabec, C. J.; Sariciftci, N. S.; Hummelen, J. C. Adv. Funct. Mater. 2001, 11, 15.

(122) Ng, S.-C.; Lu, H.-F.; Chan, H. S. O.; Fujii, A.; Laga, T.; Yoshino, K. Adv. Mater. (Weinheim, Ger.) 2000, 12, 1122.

(123) Bao, Z.; Peng, Z.; Galvin, M. E.; Chandross, E. A. Chem. Mater. 1998, $10,1201$.

(124) Son, S.; Dodabalapur, A.; Lovinger, A. J.; Galvin, M. E. Science 1995, 269, 376.

(125) Wu, A.; Jikei, M.; Kakimoto, M.-a.; Imai, Y.; Ukishima, S.; Takahashi, Y. Chem. Lett. 1994, 2319.

(126) D'Souza, F.; Gadde, S.; Zandler, M. E.; Itou, M.; Araki, Y.; Ito, O. Chem. Commun. (Cambridge, U. K.) 2004, 2276.

(127) D'Souza, F.; Deviprasad, G. R.; Zandler, M. E.; El-Khouly, M. E.; Fujitsuka, M.; Ito, O. J. Phys. Chem. A 2003, 107, 4801.

(128) D'Souza, F.; Deviprasad, G. R.; Zandler, M. E.; Hoang, V. T.; Klykov, A.; Van, S. M.; Perera, A.; El-Khouly, M. E.; Fujitsuka, M.; Ito, O. J. Phys. Chem. A 2002, 106, 3243.

(129) Kira, A.; Umeyama, T.; Matano, Y.; Yoshida, K.; Isoda, S.; Park, J. K.; Kim, D.; Imahori, H. J. Am. Chem. Soc. 2009, 131, 3198.

(130) Troshin, P. A.; Koeppe, R.; Peregudov, A. S.; Peregudova, S. M.; Egginger, M.; Lyubovskaya, R. N.; Sariciftci, N. S. Chem. Mater. 2007, 19, 5363.

(131) Hirsch, A. Top. Curr. Chem. 1999, 199, 1. 
(132) Kroto, H. W.; Heath, J. R.; O'Brien, S. C.; Curl, R. F.; Smalley, R. E. Nature (London) 1985, 318, 162.

(133) Xie, Q.; Perez-Cordero, E.; Echegoyen, L. J. Am. Chem. Soc. 1992, 114, 3978.

(134) Allemand, P. M.; Koch, A.; Wudl, F.; Rubin, Y.; Diederich, F.; Alvarez, M. M.; Anz, S. J.; Whetten, R. L. J. Am. Chem. Soc. 1991, 113, 1050.

(135) Fukuzumi, S.; Ohkubo, K.; Imahori, H.; Guldi, D. M. Chem.Eur. J. 2003, 9, 1585.

(136) Imahori, H.; El-Khouly, M. E.; Fujitsuka, M.; Ito, O.; Sakata, Y.; Fukuzumi, S. J. Phys. Chem. A 2001, 105, 325.

(137) Fukuzumi, S.; Nakanishi, I.; Suenobu, T.; Kadish, K. M. J. Am. Chem. Soc. 1999, $121,3468$.

(138) Hillman, A. R.The electrochemical quratz crystal microbalance; Wiley-VCH Verlag GmbH \& Co. KGaA: 2003; Vol. 3, p 230.

(139) Mataga, N.; Miyasaka, H. Adv. Chem. Phys. 1999, 107, 431.

(140) Monat, J. E.; Rodriguez, J. H.; McCusker, J. K. J. Phys. Chem. A 2002, 106, 7399.

(141) Fillinger, A.; Parkinson, B. A. J. Electrochem. Soc. 1999, 146, 4559.

(142) Alebbi, M.; Bignozzi, C. A.; Heimer, T. A.; Hasselmann, G. M.; Meyer, G. J. J. Phys. Chem. B 1998, 102, 7577.

(143) Nazeeruddin, M. K.; Kay, A.; Rodicio, I.; Humphry-Baker, R.; Mueller, E.; Liska, P.; Vlachopoulos, N.; Graetzel, M. J. Am. Chem. Soc. 1993, 115, 6382.

(144) Splan, K. E.; Massari, A. M.; Hupp, J. T. J. Phys. Chem. B 2004, 108, 4111.

(145) Macagno, V. A.; Giordano, M. C.; Arvia, A. J. Electrochim. Acta 1969, $14,335$.

(146) Popov, A. I.; Geske, D. H. J. Am. Chem. Soc. 1958, 80, 1340.

(147) Moss, J. A.; Stipkala, J. M.; Yang, J. C.; Bignozzi, C. A.; Meyer, G. J.; Meyer, T. J.; Wen, X.; Linton, R. W. Chem. Mater. 1998, 10, 1748.

(148) Marczak, R.; Sgobba, V.; Kutner, W.; Gadde, S.; D'Souza, F.; Guldi, D. M. Langmuir 2007, 23, 1917.

(149) Hasobe, T.; Saito, K.; Kamat, P. V.; Troiani, V.; Quu, H.; Solladie, N.; Kim, K. S.; Park, J. K.; Kim, D.; D'Souza, F.; Fukuzumi, S. J. Mater. Chem. 2007, 17, 4160. 
(150) Lehn, J.-M. Supramolecular Chemistry; Wiley-VCH Verlag GmbH \& Co. KGaA: 2006, p 199.

(151) Deisenhofer, J.; Epp, O.; Miki, K.; Huber, R.; Michel, H. Nature (London) 1986, $318,618$.

(152) Imahori, H.; Umeyama, T.; Ito, S. Acc. Chem. Res. 2009, 42, 1809.

(153) Thompson, B. C.; Frechet, J. M. J. Angew. Chem., Int. Ed. 2008, 47, 58.

(154) Goncalves, L. M.; Bermudez, V. d. Z.; Ribeiro, H. A.; Mendes, A. M. Energy Environ. Sci. 2008, 1, 655.

(155) Balzani, V.; Credi, A.; Venturi, M. Chemsuschem 2008, 1, 26.

(156) Kamat, P. V. J. Phys. Chem. C 2007, 111, 2834.

(157) Segura, J. L.; Martin, N.; Guldi, D. M. Chem. Soc. Rev. 2005, 34, 31.

(158) Imahori, H.; Fukuzumi, S. Adv. Funct. Mater. 2004, 14, 525.

(159) Gunes, S.; Neugebauer, H.; Sariciftci, N. S. Chem. Rev. 2007, 107, 1324.

(160) Subbaiyan, N. K.; Obraztsov, I.; Wijesinghe, C. A.; Tran, K.; Kutner, W.; D'Souza, F. J. Phys. Chem. C 2009, 113, 8982.

(161) Fischer, M. K. R.; Lopez-Duarte, I.; Wienk, M. M.; Martinez-Diaz, M. V.; Janssen, R. A. J.; Bauerle, P.; Torres, T. J. Am. Chem. Soc. 2009, 131, 8669.

(162) Cravino, A.; Sariciftci, N. S. J. Mat. Chem. 2002, 12, 1931.

(163) Kleverlaan, C.; Alebbi, M.; Argazzi, R.; Bignozzi, C. A.; Hasselmann, G. M.; Meyer, G. J. Inorg. Chem. 2000, 39, 1342.

(164) Imahori, H. Bull. Chem. Soc. Jpn. 2007, 80, 621.

(165) Zagal, J. H.; Bedioui, F.; Dodelet, J.-P. $N_{4}$ macrocyclic metal compound; Springer: New York, 2006.

(166) Wasielewski, M. R. Acc. Chem. Res. 2009, 42, 1910.

(167) Subbaiyan, N. K.; Wijesinghe, C. A.; D'Souza, F. J. Am. Chem. Soc. 2009, 131, 14646.

(168) Hagfeldt, A.; Boschloo, G.; Sun, L.; Kloo, L.; Pettersson, H. Chem. Rev. 2010, $110,6595$. 
(169) Radivojevic, I.; Varotto, A.; Farley, C.; Drain, C. M. Ener. Environ. Sci. 2010, 3, 1897.

(170) Morandeira, A.; Lopez-Duarte, I.; Martinez-Diaz, M. V.; O'Regan, B.; Shuttle, C.; Haji-Zainulabidin, N. A.; Torres, T.; Palomares, E.; Durrant, J. R. J. Am. Chem. Soc. 2007, 129, 9250.

(171) Varfolomeev, S. D.; Krylova, L.; Zaikov, G. E. Molecular and nanoscale systems for energy conversion; Nova Science Publishers: New York.

(172) Smith, K. M. Porphyrins and Metalloporphyrins ( New Ed. based on original vol. by J.E. Falk); Elsevier, 1975.

(173) D'Souza, F.; El-Khouly, M. E.; Gadde, S.; McCarty, A. L.; Karr, P. A.; Zandler, M. E.; Araki, Y.; Ito, O. J. Phys. Chem. B 2005, 109, 10107.

(174) D'Souza, F.; Deviprasad, G. R.; Zandler, M. E.; Hoang, V. T.; Klykov, A.; VanStipdonk, M.; Perera, A.; El-Khouly, M. E.; Fujitsuka, M.; Ito, O. J. Phys. Chem. A 2002, 106, 3243.

(175) Yan, S. G.; Prieskorn, J. S.; Kim, Y.; Hupp, J. T. J. Phys. Chem. B 2000, 104, 10871.

(176) Hwang, S.; Lee, J. H.; Park, C.; Lee, H.; Kim, C.; Park, C.; Lee, M.-H.; Lee, W.; Park, J.; Kim, K.; Park, N.-G.; Kim, C. Chem. Commun. (Cambridge, U. K.) 2007, 4887.

(177) Hamann, T. W.; Ondersma, J. W. Ener. Environ. Sci. 2011, 4, 370.

(178) Hauch, A.; Georg, A. Electrochim. Acta. 2001, 46, 3457.

(179) Sgobba, V.; Guldi, D. M. Chem. Soc. Rev. 2009, 38, 165.

(180) Hardin, B. E.; Hoke, E. T.; Armstrong, P. B.; Yum, J.-H.; Comte, P.; Torres, T.; Frechet, J. M. J.; Nazeeruddin, M. K.; Gratzel, M.; McGehee, M. D. Nat. Photonics 2009, 3, 667.

(181) Fukuzumi, S.; Kojima, T. J. Mater. Chem. 2008, 18, 1427.

(182) Figueira-Duarte, T. M.; Gegout, A.; Nierengarten, J.-F. Chem. Commun. (Cambridge, U. K.) 2007, 109.

(183) Fukuzumi, S. Prog. Inorg. Chem. 2009, 56, 49.

(184) Yocum, C. F. Coord. Chem. Rev. 2008, 252, 296.

(185) Utschig, L. M.; Thurnauer, M. C. Acc. Chem. Res. 2004, 37, 439. 
(186) D'Souza, F.; Subbaiyan, N. K.; Xie, Y.; Hill, J. P.; Ariga, K.; Ohkubo, K.; Fukuzumi, S. J. Am. Chem. Soc. 2009, 131, 16138.

(187) Xie, Y.; Hill, J. P.; Schumacher, A. L.; Sandanayaka, A. S. D.; Araki, Y.; Karr, P. A.; Labuta, J.; D'Souza, F.; Ito, O.; Anson, C. E.; Powell, A. K.; Ariga, K. J. Phys. Chem. C 2008, 112, 10559.

(188) Hill, J. P.; Schumacher, A. L.; D'Souza, F.; Labuta, J.; Redshaw, C.; Elsegood, M. R. J.; Aoyagi, M.; Nakanishi, T.; Ariga, K. Inorg. Chem. 2006, 45, 8288.

(189) Schumacher, A. L.; Hill, J. P.; Ariga, K.; D'Souza, F. Electrochem. Commun. 2007, 9, 2751.

(190) Palmer, B. D.; Thompson, A. M.; Sutherland, H. S.; Blaser, A.; Kmentova, I.; Franzblau, S. G.; Wan, B.; Wang, Y.; Ma, Z.; Denny, W. A. J. Med. Chem. 2010, 53, 282.

(191) Hill, J. P.; Hewitt, I. J.; Anson, C. E.; Powell, A. K.; McCarty, A. L.; Karr, P. A.; Zandler, M. E.; D'Souza, F. J. Org. Chem. 2004, 69, 5861.

(192) Dubois, D.; Moninot, G.; Kutner, W.; Jones, M. T.; Kadish, K. M. J. Phys. Chem. $1992,96,7137$.

(193) El-Khouly, M. E.; Ju, D. K.; Kay, K.-Y.; D'Souza, F.; Fukuzumi, S. Chem.Eur. J. 2010, 16, 6193.

(194) D'Souza, F.; Ito, O. Chem. Commun. (Cambridge, U. K.) 2009, 4913.

(195) Chitta, R.; D'Souza, F. J. Mater. Chem. 2008, 18, 1440.

(196) Winters, M. U.; Kaernbratt, J.; Blades, H. E.; Frampton, M. J.; Anderson, H. L.; Albinsson, B. Chem.Eur. J. 2007, 13, 7385.

(197) Saha, S.; Flood, A. H.; Stoddart, J. F.; Impellizzeri, S.; Silvi, S.; Venturi, M.; Credi, A. J. Am. Chem. Soc. 2007, 129, 12159.

(198) Straight, S. D.; Andreasson, J.; Kodis, G.; Moore, A. L.; Moore, T. A.; Gust, D. J. Am. Chem. Soc. 2005, 127, 2717.

(199) Fukuzumi, S.; Imahori, H. Biomimetic electron-transfer chemistry of porphyrins and metalloporphyrins; Wiley-VCH Verlag GmbH: 2001; Vol. 2, p 927.

(200) Paddon-Row, M. N. Electron and energy transfer; Wiley-VCH Verlag GmbH \& Co. KGaA: 2000, p 267. 
(201) Figueira-Duarte, T. M.; Rio, Y.; Listorti, A.; Delavaux-Nicot, B.; Holler, M.; Marchioni, F.; Ceroni, P.; Armaroli, N.; Nierengarten, J.-F. New J. Chem. 2008, $32,54$.

(202) Ajayaghosh, A.; Praveen, V. K.; Vijayakumar, C. Chem. Soc. Rev. 2008, 37, 109.

(203) Kelley, R. F.; Shin, W. S.; Rybtchinski, B.; Sinks, L. E.; Wasielewski, M. R. J. Am. Chem. Soc. 2007, 129, 3173.

(204) Baranoff, E.; Barigelletti, F.; Bonnet, S.; Collin, J.-P.; Flamigni, L.; Mobian, P.; Sauvage, J.-P. Struct. Bonding (Berlin, Ger.) 2007, 123, 41.

(205) Kodis, G.; Terazono, Y.; Liddell, P. A.; Andreasson, J.; Garg, V.; Hambourger, M.; Moore, T. A.; Moore, A. L.; Gust, D. J. Am. Chem. Soc. 2006, 128, 1818.

(206) Burquel, A.; Lemaur, V.; Beljonne, D.; Lazzaroni, R.; Cornil, J. J. Phys. Chem. A 2006, 110, 3447.

(207) Giacalone, F.; Segura, J. L.; Martin, N.; Ramey, J.; Guldi, D. M. Chem. Eur. J. 2005, 11, 4819.

(208) Imahori, H.; Sekiguchi, Y.; Kashiwagi, Y.; Sato, T.; Araki, Y.; Ito, O.; Yamada, H.; Fukuzumi, S. Chem. Eur. J. 2004, 10, 3184.

(209) Beckers, E. H. A.; Meskers, S. C. J.; Schenning, A. P. H. J.; Chen, Z.; Wuerthner, F.; Janssen, R. A. J. J. Phys. Chem. A 2004, 108, 6933.

(210) Paddon-Row, M. N. Aust. J. Chem. 2003, 56, 729.

(211) Neuteboom, E. E.; Meskers, S. C. J.; Van, H. P. A.; Van, D. J. K. J.; Meijer, E. W.; Janssen, R. A. J.; Dupin, H.; Pourtois, G.; Cornil, J.; Lazzaroni, R.; Bredas, J.-L.; Beljonne, D. J. Am. Chem. Soc. 2003, 125, 8625.

(212) Ohkubo, K.; Fukuzumi, S. Bull. Chem. Soc. Jpn. 2009, 82, 303.

(213) Fukuzumi, S. Eur. J. Inorg. Chem. 2008, 1351.

(214) Fukuzumi, S. Bull. Chem. Soc. Jpn. 2006, 79, 177.

(215) Kaunisto, K.; Chukharev, V.; Tkachenko, N. V.; Efimov, A.; Lemmetyinen, H. J. Phys. Chem. C 2009, 113, 3819.

(216) Schuster, D. I.; Li, K.; Guldi, D. M.; Palkar, A.; Echegoyen, L.; Stanisky, C.; Cross, R. J.; Niemi, M.; Tkachenko, N. V.; Lemmetyinen, H. J. Am. Chem. Soc. 2007, 129, 15973.

(217) Schuster, D. I.; Li, K.; Guldi, D. M. C. R. Chim. 2006, 9, 892. 
(218) Guldi, D. M.; Rahman, G. M. A.; Sgobba, V.; Ehli, C. Chem. Soc. Rev. 2006, 35, 471.

(219) Isosomppi, M.; Tkachenko, N. V.; Efimov, A.; Lemmetyinen, H. J. Phys. Chem. A 2005, 109, 4881.

(220) Xiao, S.; Li, Y.; Li, Y.; Zhuang, J.; Wang, N.; Liu, H.; Ning, B.; Liu, Y.; Lu, F.; Fan, L.; Yang, C.; Li, Y.; Zhu, D. J. Phys. Chem. B 2004, 108, 16677.

(221) Lu, F.; Xiao, S.; Li, Y.; Liu, H.; Li, H.; Zhuang, J.; Liu, Y.; Wang, N.; He, X.; Li, X.; Gan, L.; Zhu, D. Macromolecules 2004, 37, 7444.

(222) Smirnov, S. N.; Liddell, P. A.; Vlassiouk, I. V.; Teslja, A.; Kuciauskas, D.; Braun, C. L.; Moore, A. L.; Moore, T. A.; Gust, D. J. Phys. Chem. A 2003, 107, 7567.

(223) Balbinot, D.; Atalick, S.; Guldi, D. M.; Hatzimarinaki, M.; Hirsch, A.; Jux, N. J. Phys. Chem. B 2003, 107, 13273.

(224) Milanesio, M. E.; Gervaldo, M.; Otero, L. A.; Sereno, L.; Silber, J. J.; Durantini, E. N. J. Phys. Org. Chem. 2002, 15, 844.

(225) Liddell, P. A.; Kuciauskas, D.; Sumida, J. P.; Nash, B.; Nguyen, D.; Moore, A. L.; Moore, T. A.; Gust, D. J. Am. Chem. Soc. 1997, 119, 1400.

(226) Bell, T. D. M.; Smith, T. A.; Ghiggino, K. P.; Ranasinghe, M. G.; Shepard, M. J.; Paddon-Row, M. Chem. Phys. Lett. 1997, 268, 223.

(227) Kuciauskas, D.; Lin, S.; Seely, G. R.; Moore, A. L.; Moore, T. A.; Gust, D.; Drovetskaya, T.; Reed, C. A.; Boyd, P. D. W. J. Phys. Chem. 1996, 100, 15926.

(228) Popelkova, H.; Commet, A.; Kuntzleman, T.; Yocum, C. F. Biochemistry 2008, 47, 12593.

(229) Gossas, T.; Danielson, U. H. Biochem. J. 2006, 398, 393.

(230) Zak, O.; Ikuta, K.; Aisen, P. Biochemistry 2002, 41, 7416.

(231) Burkhard, P.; Tai, C.-H.; Jansonius, J. N.; Cook, P. F. J. Mol. Biol. 2000, 303, 279.

(232) Sessler, J. L.; Gale, P. A. Calixpyrroles: novel anion and nuetral substrate receptors; Academic Press: 2000; Vol. 6, p 257.

(233) Siegbahn, P. E. M.; Crabtree, R. H. J. Am. Chem. Soc. 1999, 121, 117.

(234) Jou, R.; Cowan, J. A. J. Am. Chem. Soc. 1991, 113, 6685. 
(235) Guskov, A.; Kern, J.; Gabdulkhakov, A.; Broser, M.; Zouni, A.; Saenger, W. Nat. Struct. Mol. Biol. 2009, 16, 334.

(236) Murray, J. W.; Maghlaoui, K.; Kargul, J.; Ishida, N.; Lai, T.-L.; Rutherford, A. W.; Sugiura, M.; Boussac, A.; Barber, J. Ener. Environ. Sci. 2008, 1, 161.

(237) Popelkova, H.; Yocum, C. F. Photosynth. Res. 2007, 93, 111.

(238) van, G. H. J.; Yocum, C. F. Adv. Photosynth. Respir. 2005, 22, 307.

(239) Barber, J. Q. Rev. Biophys. 2003, 36, 71.

(240) Wincencjusz, H.; Yocum, C. F.; van, G. H. J. Biochemistry 1999, 38, 3719.

(241) Lindberg, K.; Vaenngaard, T.; Andreasson, L. E. Photosynth. Res. 1993, 38, 401.

(242) Hill, J. P.; Schmitt, W.; McCarty, A. L.; Ariga, K.; D'Souza, F. Eur. J. Org. Chem. 2005, 2893.

(243) Ohkubo, K.; Kotani, H.; Shao, J.; Ou, Z.; Kadish, K. M.; Li, G.; Pandey, R. K.; Fujitsuka, M.; Ito, O.; Imahori, H.; Fukuzumi, S. Angew. Chem., Int. Ed. 2004, 43, 853.

(244) Kobori, Y.; Shibano, Y.; Endo, T.; Tsuji, H.; Murai, H.; Tamao, K. J. Am. Chem. Soc. 2009, 131, 1624.

(245) Kobori, Y.; Yamauchi, S.; Akiyama, K.; Tero-Kubota, S.; Imahori, H.; Fukuzumi, S.; Norris, J. R., Jr. Proc. Natl. Acad. Sci. U. S. A. 2005, 102, 10017.

(246) Mori, Y.; Sakaguchi, Y.; Hayashi, H. J. Phys. Chem. A 2002, 106, 4453.

(247) Wegner, M.; Fischer, H.; Grosse, S.; Vieth, H. M.; Oliver, A. M.; Paddon-Row, M. N. Chem. Phys. 2001, 264, 341.

(248) Nakanishi, I.; Fukuzumi, S.; Konishi, T.; Ohkubo, K.; Fujitsuka, M.; Ito, O.; Miyata, N. J. Phys. Chem. B 2002, 106, 2372.

(249) Kim, S. K.; Sessler, J. L. Chem. Soc. Rev. 2010, 39, 3784.

(250) Davis, A. P.; Sheppard, D. N.; Smith, B. D. Chem. Soc. Rev. 2007, 36, 348.

(251) Sessler, J. L.; Gale, P. A.; Cho, W.-S. Anion receptor chemistry; Royal Society of Chemistry: Cambridge, 2006.

(252) Atwood, J. L.; Steed, J. W. Encyclopedia of supramolecular chemistry; M. Dekker: New York, 2004. 
(253) Beer, P. D.; Gale, P. A. Angew. Chem., Int. Ed. 2001, 40, 486.

(254) Bianchi, A.; Bowman-James, K.; García-España, E. Supramolecular chemistry of anions; Wiley-VCH: New York, 1997.

(255) Hill, J. P.; El-Khouly, M. E.; Charvet, R.; Subbaiyan, N. K.; Ariga, K.; Fukuzumi, S.; D'Souza, F. Chem. Commun. (Cambridge, U. K.) 2010, 46, 7933.

(256) Schumacher, A. L.; Sandanayaka, A. S. D.; Hill, J. P.; Ariga, K.; Karr, P. A.; Araki, Y.; Ito, O.; D'Souza, F. Chem. Eur. J. 2007, 13, 4628.

(257) Hill, J. P.; Sandanayaka, A. S. D.; McCarty, A. L.; Karr, P. A.; Zandler, M. E.; Charvet, R.; Ariga, K.; Araki, Y.; Ito, O.; D'Souza, F. Eur. J. Org. Chem. 2006, 595.

(258) Hill, J. P.; Ariga, K.; Schumacher, A. L.; Karr, P. A.; D'Souza, F. J. Porphyrins Phthalocyanines 2007, 11, 390.

(259) Kroon, J. M.; Bakker, N. J.; Smit, H. J. P.; Liska, P.; Thampi, K. R.; Wang, P.; Zakeeruddin, S. M.; Gratzel, M.; Hinsch, A.; Hore, S.; Wuerfel, U.; Sastrawan, R.; Durrant, J. R.; Palomares, E.; Pettersson, H.; Gruszecki, T.; Walter, J.; Skupien, K.; Tulloch, G. E. Prog. Photovoltaics 2007, 15, 1.

(260) O'Regan, B.; Gratzel, M. Nature (London) 1991, 353, 737.

(261) Martinez-Diaz, M. V.; de, I. T. G.; Torres, T. Chem. Commun. (Cambridge, U. K.) 2010, 46, 7090 .

(262) Delgado, J. L.; Bouit, P.-A.; Filippone, S.; Herranz, M. A.; Martin, N. Chem. Commun. (Cambridge, U. K.) 2010, 46, 4853.

(263) Pagliaro, M.; Palmisano, G.; Ciriminna, R.; Loddo, V. Energy Environ. Sci. 2009, 2, 838.

(264) Kamat, P. V.; Schatz, G. C. J. Phys. Chem. C 2009, 113, 15473.

(265) Baxter, J.; Bian, Z.; Chen, G.; Danielson, D.; Dresselhaus, M. S.; Fedorov, A. G.; Fisher, T. S.; Jones, C. W.; Maginn, E.; Kortshagen, U.; Manthiram, A.; Nozik, A.; Rolison, D. R.; Sands, T.; Shi, L.; Sholl, D.; Wu, Y. Ener. Environ. Sci. 2009, 2, 559.

(266) Umeyama, T.; Imahori, H. Ener. Environ. Sci. 2008, 1, 120.

(267) Hamann, T. W.; Jensen, R. A.; Martinson, A. B. F.; Van, R. H.; Hupp, J. T. Ener. Environ. Sci. 2008, 1, 66.

(268) Snaith, H. J.; Schmidt-Mende, L. Adv. Mater. (Weinheim, Ger.) 2007, $19,3187$. 
(269) Talapin, D. V.; Murray, C. B. Science (Washington, DC, U. S.) 2005, 310, 86.

(270) Tulloch, G. E. J. Photochem. Photobiol., A 2004, 164, 209.

(271) Reynal, A.; Forneli, A.; Palomares, E. Ener. Environ. Sci. 2010, 3, 805.

(272) Radivojevic, I.; Varotto, A.; Farley, C.; Drain, C. M. Ener. Environ. Sci. 2010, 3, 1897.

(273) Liang, M.; Xu, W.; Cai, F.; Chen, P.; Peng, B.; Chen, J.; Li, Z. J. Phys. Chem. C 2007, 111, 4465 .

(274) Hagberg, D. P.; Edvinsson, T.; Marinado, T.; Boschloo, G.; Hagfeldt, A.; Sun, L. Chem. Commun. (Cambridge, U. K.) 2006, 2245.

(275) Nazeeruddin, M. K.; De, A. F.; Fantacci, S.; Selloni, A.; Viscardi, G.; Liska, P.; Ito, S.; Takeru, B.; Gratzel, M. J. Am. Chem. Soc. 2005, 127, 16835.

(276) Polo, A. S.; Itokazu, M. K.; Murakami, I. N. Y. Coord. Chem. Rev. 2004, 248, 1343.

(277) Kitiyanan, A.; Yoshikawa, S. Mater. Lett. 2005, 59, 4038.

(278) Deb, S. K. Sol. Energy Mater. Sol. Cells 2005, 88, 1.

(279) Huang, M. H.; Mao, S.; Feick, H.; Yan, H.; Wu, Y.; Kind, H.; Weber, E.; Russo, R.; Yang, P. Science 2001, 292, 1897.

(280) Tennakone, K.; Kumara, G. R. R. A.; Kottegoda, I. R. M.; Perera, V. P. S. Chem. Commun. (Cambridge) 1999, 15.

(281) Sayama, K.; Sugihara, H.; Arakawa, H. Chem. Mater. 1998, 10, 3825.

(282) Tian, H.; Yang, X.; Pan, J.; Chen, R.; Liu, M.; Zhang, Q.; Hagfeldt, A.; Sun, L. Adv. Funct. Mater. 2008, 18, 3461.

(283) Hagberg, D. P.; Yum, J.-H.; Lee, H.; De, A. F.; Marinado, T.; Karlsson, K. M.; Humphry-Baker, R.; Sun, L.; Hagfeldt, A.; Gratzel, M.; Nazeeruddin, M. K. J. Am. Chem. Soc. 2008, 130, 6259 .

(284) Erten-Ela, S.; Yilmaz, M. D.; Icli, B.; Dede, Y.; Icli, S.; Akkaya, E. U. Org. Lett. 2008, 10, 3299.

(285) Choi, H.; Baik, C.; Kang, S. O.; Ko, J.; Kang, M.-S.; Nazeeruddin, M. K.; Graetzel, M. Angew. Chem., Int. Ed. 2008, 47, 327. 
(286) Kay, A.; Graetzel, M. Chem. Mater. 2002, 14, 2930.

(287) Dang, X.; Hupp, J. T. J. Photochem. Photobiol., A 2001, 143, 251.

(288) Liu, D.; Kamat, P. V. J. Electrochem. Soc. 1995, 142, 835.

(289) Kim, Y. S.; Liang, K.; Law, K. Y.; Whitten, D. G. J. Phys. Chem. 1994, 98, 984.

(290) Bedja, I.; Hotchandani, S.; Carpentier, R.; Fessenden, R. W.; Kamat, P. V. J. Appl. Phys. 1994, 75, 5444.

(291) Krishnan, M.; Zhang, X.; Bard, A. J. J. Am. Chem. Soc. 1984, 106, 7371.

(292) Kamat, P. V.; Fox, M. A.; Fatiadi, A. J. J. Am. Chem. Soc. 1984, 106, 1191.

(293) Miyasaka, T.; Watanabe, T.; Fujishima, A.; Honda, K. J. Am. Chem. Soc. 1978, $100,6657$.

(294) Heyrovsky, M.; Jirkovsky, J.; Mueller, B. R. Langmuir 1995, 11, 4293.

(295) Snaith, H. J.; Ducati, C. Nano Lett. 2010, 10, 1259.

(296) Senevirathna, M. K. I.; Pitigala, P. K. D. D. P.; Premalal, E. V. A.; Tennakone, K.; Kumara, G. R. A.; Konno, A. Sol. Energy Mater. Sol. Cells 2007, 91, 544.

(297) Ito, S.; Makari, Y.; Kitamura, T.; Wada, Y.; Yanagida, S. J. Mater. Chem. 2004, $14,385$.

(298) Pasternack, R. F.; Francesconi, L.; Raff, D.; Spiro, E. Inorg. Chem. 1973, 12, 2606.

(299) Hambright, P.; Fleisher, E. B. Inorg. Chem. 1970, 9, 1757.

(300) Chernia, Z.; Gill, D. Langmuir 1999, 15, 1625.

(301) Lakowicz, J. R. Principles of fluorescence spectroscopy; Springer-Verlag US: Boston, MA, 2006.

(302) Kadish, K. M.; Van, C. E.; Royal, G. Electrochemistry of metalloporphyrins in nonaqueous media; Academic Press: 2000; Vol. 8, p 1.

(303) Kalyanasundaram, K.; Neumann-Spallart, M. J. Phys. Chem. 1982, 86, 5163.

(304) Ford, W. E.; Rodgers, M. A. J. J. Phys. Chem. 1994, 98, 3822. 
(305) Seery, M. K.; Guerin, L.; Forster, R. J.; Gicquel, E.; Hultgren, V.; Bond, A. M.; Wedd, A. G.; Keyes, T. E. J. Phys. Chem. A 2004, 108, 7399.

(306) Prasittichai, C.; Hupp, J. T. J. Phys. Chem. Lett. 2010, 1, 1611.

(307) Ito, S.; Liska, P.; Comte, P.; Charvet, R.; Pechy, P.; Bach, U.; Schmidt-Mende, L.; Zakeeruddin, S. M.; Kay, A.; Nazeeruddin, M. K.; Graetzel, M. Chem. Commun. (Cambridge, U. K.) 2005, 4351.

(308) Liu, D.; Fessenden, R. W.; Hug, G. L.; Kamat, P. V. J. Phys. Chem. B 1997, 101, 2583.

(309) Gubbala, S.; Russell, H. B.; Shah, H.; Deb, B.; Jasinski, J.; Rypkema, H.; Sunkara, M. K. Ener. Environ. Sci. 2009, 2, 1302.

(310) Green, A. N. M.; Palomares, E.; Haque, S. A.; Kroon, J. M.; Durrant, J. R. J. Phys. Chem. B 2005, 109, 12525.

(311) Kelly, J. J.; Hens, Z.; Vanmaekelbergh, D.; Hensalso, Z. Photoelectrochemical systems characterization; Wiley-VCH Verlag GmbH \& Co. KGaA: 2003; Vol. 6, p 59.

(312) Halme, J.; Vahermaa, P.; Miettunen, K.; Lund, P. Adv. Mater. (Weinheim, Ger.) 2010, 22, E210.

(313) Guo, X.-Z.; Luo, Y.-H.; Li, C.-H.; Qin, D.; Li, D.-M.; Meng, Q.-B. Curr. Appl. Phys.

(314) Wang, Q.; Moser, J.-E.; Graetzel, M. J. Phys. Chem. B 2005, 109, 14945.

(315) Fungo, F.; Otero, L.; Durantini, E. N.; Silber, J. J.; Sereno, L. E. J. Phys. Chem. B 2000, 104, 7644 .

(316) Gratzel, M. Acc. Chem. Res. 2009, 42, 1788.

(317) Hardin, B. E.; Hoke, E. T.; Armstrong, P. B.; Yum, J. H.; Comte, P.; Torres, T.; Frechet, J. M. J.; Nazeeruddin, M. K.; Gratzel, M.; McGehee, M. D. Nat. Photonics 2009, 3, 406.

(318) Imahori, H.; Norieda, H.; Yamada, H.; Nishimura, Y.; Yamazaki, I.; Sakata, Y.; Fukuzumi, S. J. Am. Chem. Soc. 2001, 123, 100.

(319) Delbaere, S.; Vermeersch, G.; Frigoli, M.; Mehl, G. H. Org. Lett. 2010, $12,4090$.

(320) Subbaiyan, N. K.; Wijesinghe, C. A.; D'Souza, F. J. Am. Chem. Soc. 2009, 131, 14646. 
(321) Jordan, P.; Fromme, P.; Witt, H. T.; Klukas, O.; Saenger, W.; Krauss, N. Nature 2001, 411, 909.

(322) D'Souza, F.; Ito, O. Chem. Soc. Rev. 2012, 41, 86.

(323) Kamat, P. V. J. Phys. Chem. Lett. 2011, 2, 242.

(324) Hasobe, T. Phys. Chem. Chem. Phys. 2010, 12, 44.

(325) Imahori, H.; Liu, J. C.; Hotta, H.; Kira, A.; Umeyama, T.; Matano, Y.; Li, G. F.; Ye, S.; Isosomppi, M.; Tkachenko, N. V.; Lemmetyinen, H. J. Phys. Chem. B 2005, 109, 18465.

(326) Nasr, C.; Hotchandani, S.; Kim, W. Y.; Schmehl, R. H.; Kamat, P. V. J. Phys. Chem. B 1997, 101, 7480.

(327) Subbaiyan, N. K.; Maligaspe, E.; D'Souza, F. Acs. Appl. Mater. Inter. 2011, 3, 2368.

(328) Fermin, D. J.; Duong, H. D.; Ding, Z. F.; Brevet, P. F.; Girault, H. H. Electrochem. Commun. 1999, 1, 29.

(329) Ariga, K.; Lvov, Y.; Kunitake, T. J. Am. Chem. Soc. 1997, 119, 2224.

(330) Fathalla, M.; Neuberger, A.; Li, S. C.; Schmehl, R.; Diebold, U.; Jayawickramarajah, J. J. Am. Chem. Soc. 2010, 132, 9966.

(331) Schwab, A. D.; Smith, D. E.; Bond-Watts, B.; Johnston, D. E.; Hone, J.; Johnson, A. T.; de Paula, J. C.; Smith, W. F. Nano Lett. 2004, 4, 1261.

(332) Bai, F.; Sun, Z.; Wu, H.; Haddad, R. E.; Coker, E. N.; Huang, J. Y.; Rodriguez, M. A.; Fan, H. Nano Lett. 2011, 11, 5196.

(333) TranThi, T. H. Coordn. Chem. Rev. 1997, 160, 53.

(334) Lipskier, J. F.; Tran-Thi, T. H. Inorg. Chem. 1993, 32, 722.

(335) Benesi, H. A.; Hildebrand, J. H. J. Am. Chem. Soc. 1949, 71, 2703.

(336) Takeo Shimidzu, T. I. Chem. Lett. 1981, 853. 